\title{
Term Analysis of the First Spectrum of Rhenium (Re I)
}

\author{
P. F. A. Klinkenberg, ${ }^{1}$ W. F. Meggers, R. Velasco, ${ }^{2}$ and M. A. Catalán ${ }^{2}$
}

\begin{abstract}
With the aid of new data on wavelengths, intensities, and the Zeeman effect, the structural analysis of the first spectrum of rhenium (Re I) has been extended to include 2,764 lines that are explained as transitions among 282 atomic energy levels. This analysis accounts for 90 percent of the total observed intensity, although only 64 percent of the total number of lines has been classified. From the Zeeman effect, magnetic splitting factors have been derived for 75 percent of the levels, and nearly 40 percent of these have been grouped into designated spectral terms ascribed to specific configurations of electrons. The normal state of neutral Re atoms is $5 d^{5} 6 s^{2}{ }^{6} \mathrm{~S}_{23,3}$, and the ionization limit is approximately 63530 kaysers or 7.87 electron volts
\end{abstract}

\section{Introduction}

For a quarter century the atomic emission spectra of rhenium have been investigated intermittently by several researchers, and now a full report on the present status of the analysis and interpretation of the first spectrum of rhenium (Re I) is presented in this paper.

In 1931 the first useful description (2500.59 to $8797.6 \mathrm{~A}$ ) and partial analysis of Re $\mathrm{I}$ was published by Meggers [1], ${ }^{3}$ who classified about 500 lines as combinations of 115 atomic energy levels, 24 of which were assigned spectral term symbols. In a second paper [2] the spectrum was extended to longer waves (10639.44 A), and 5 additional metastable energy levels were reported. In 1947 the analysis of Re I was considerably extended by Klinkenberg [3] who used new data in the MIT Wavelength Tables [4], principally between 2500 and $2000 \mathrm{~A}$, and increased the number of levels to 172 and the number of classified lines to 1,171. In a second paper [5], he increased the number of levels to 221 , the number of classified lines to 1,624 , and identified many terms (of even parity) with electron configurations. Since 1946 Velasco has also worked independently on Re I; in 1949 he [6] pointed out some discrepancies between his levels and Klinkenberg's, and in 1952 he [7] reported measurements between 2000 and $1500 \mathrm{~A}$, revised some levels reported by Meggers [1], classified about 150 lines with 29 new levels, and correlated many spectral terms (even and odd) with electron configurations.

The above mentioned contributions to the interpretation of Re I were all made without the benefit of Zeeman effect; they were based entirely on wave numbers and intensities, and were guided by comparisons with other analyzed spectra, and by quantum theoretical predictions. In order to make further progress with the analysis of Re I it was felt necessary to make new descriptions of the arc and spark spectra of rhenium and to investigate the Zeeman effect.

A new description of the arc and spark spectra of rhenium was supplied by Meggers [8], who used solid electrodes of pure rhenium and obtained wave-

1 Zeeman Laboratorium, Amsterdam, The Netherlands.

2 Instituto de Optica, Madrid, Spain.

3 Figures in brackets indicate the literature references on page 326. lengths and intensities for approximately 6,000 lines of which about 4,200 were assigned to Re I. These data, supplemented by those of Velasco [7] from arc spectrograms made by Catalán with a vacuum spectrograph at Princeton University, comprise an improved list of $R e$ I lines ranging in wavelength from about $1700 \mathrm{~A}$ in the extreme ultraviolet to $12000 \mathrm{~A}$, the photographic limit in the infrared. This list was used by Klinkenberg in making this final attack on the Re I spectrum; new levels were found and the relative values of all atomic energy levels were recalculated.

The splitting of spectral lines by magnetic fields (Zeeman effect) is extremely useful in analyses of complex spectra because it reveals (by the number of components) the inner-quantum numbers $(J)$ of both levels, and often identifies (by the splitting factors, $g$ ) the term types $(L)$ and multiplicities $(r)$. At the Massachusetts Institute of Technology the Bitter magnet [9] was constructed and operated for this purpose, and the MIT wavelength project [10] included Zeeman spectrograms of rhenium. These spectrograms were made just before World War II and during the war years they were in the possession of graduate students who had no opportunity to measure and interpret them. They were finally loaned to the National Bureau of Standards where they were examined and stored. In 1949, through the courtesy of G. R. Harrison, Meggers made greatly superior Zeeman spectrograms of rhenium in the Spectroscopy Laboratory at MIT. These were hand-measured at the Bureau, the wavelengths of Zeeman components were computed, and some resolved patterns gave a start on the analysis of Re II in 1950. At that time the record books were turned over to Catalán and Velasco, who fully exploited the Zeeman data for the determination of $J$-values and calculation of $g$-factors for energy levels in the first two spectra of rhenium, Re $\mathrm{I}$ and Re II. A preliminary report [11] on these results indicated that $g$-values had been derived for 164 Re I levels, and for 39 Re II levels. The combined efforts of three research groups in accumulating and analyzing spectral data for Re I have improved and extended the analysis to include 2764 classified lines arising from permitted transitions among 51 low even, 20 high even, and 211 middle odd levels, with $g$-values evaluated for 75 percent of all levels. 
The details concerning established levels and classified lines are presented in tables below, and are followed by a discussion of the experimental and theoretical structure of the Re I spectrum.

\section{Atomic Energy Levels of Re I}

Before this analysis could proceed it was necessary to convert the newly measured wavelengths to vacuum. wave numbers, and for this purpose the Edlén formula for the dispersion of standard air [12] was used as recommended in 1952 by the Joint Commission for Spectroscopy [13]. Then all previously discovered atomic energy levels of Re I were recalculated with the new data so that old and new levels would be consistent. Many new lines in the list could be immediately classified as transitions between known levels, and nearly all levels adopted previously $[3,5]$ were confirmed. The exceptions are $334_{1 / 2}, \quad 3389_{1 / 2}, \quad 3481_{5 / 2}, \quad 3575_{1 / 2}, 3641_{5 / 2}, \quad 3801_{1 / 2}$, $3955_{9 / 2}, 5366_{7 / 2}, 5626_{1 / 2}$, and $5829_{11 / 2}$, four of which had been characterized as questionable before [5]. All the rejected levels belong to the odd group. The analysis was continued with the remaining unclassified $\mathrm{Re}$ I lines with the result that 12 low even levels, 3 high even levels, and 46 odd levels could be added to those previously found; they are reported for the first time in this paper. In addition, 11 of the odd levels proposed by Velasco [7], and 8 qualified as provisional by Klinkenberg [5], have been confirmed. The total number of established atomic energy levels resulting from this analysis of the Re I spectrum is now 282 which is the sum of 51 low even, 20 high even, and 211 odd levels. These energy levels are listed in table 1 in which successive columns display an abbreviated designation, the level value (in $\mathrm{K}=\mathrm{cm}^{-1}$ ), the inner quantum number $J$, the magnetic splitting factor $g$, and the origin of the level. The abridged designation of levels is according to the system adopted by Klinkenberg [3] in 1947: "The odd and high even levels are given by the first 4 digits, the ground levels (which are more widely spaced) by the first 3 digits; however where ambiguities occur an alteration by one or more units of the last figure is allowed. The odd terms are printed in italics".

The $J$-values have been fixed either by the Zeeman effect or by the combinations, and a number of the $J$-values in earlier papers have been corrected in this analysis: for level 261 the value $5 / 2$ has been changed to $3 / 2$; for level $278,5 / 2$ to $3 / 2$; for level $3991,9 / 2$ to $11 / 2$; for level $4081,3 / 2$ to $1 / 2$; for level $4614,3 / 2$ to $1 / 2$; for level $4637,11 / 2$ to $9 / 2$; for level $509 \%, 3 / 2$ to $5 / 2$; for level 5221, $3 / 2$ ? to $5 / 2$; and for level 5482,3/2 to 5/2. An important contribution of the Zeeman effect has been the absolute determination of the $J$-values of the levels for lines whose magnetic splitting has been completely observed and resolved. In all previous analyses of the Re I spectrum the $J$-values were assumed to be those required by theory for the identified terms and the remainder were then derived from the level combinations according to the selection rule that $\Delta J=0$ or \pm 1 for permitted transitions.
The Zeeman-effect spectrograms were made with magnetic field intensity of 81,000 oersteds, the field intensity being determined from the splitting of impurity lines of $\mathrm{Ag}, \mathrm{Cu}, \mathrm{Mg}$, and $\mathrm{Ca}$. Their measurement was complicated by hyperfine structures, and by dissymmetries usually due to partial Paschen-Back effect. These difficulties are illustrated respectively in the two small samples of Re I spectra reproduced in figure 1 . The upper strip of spectrum shows the resonance lines ${ }^{6} \mathrm{~S}-{ }^{6} \mathrm{P}^{\circ}$; the Zeeman components are complex and wide with hyperfine structure except when the magnetic quantum number is $1 / 2$. The lower strip displays a portion of the Re I spectrum in which most Zeeman patterns are so badly perturbed that they cannot be interpreted without great difficulty. When the classification of these lines is examined in table 2 it is obvious that the asymmetrical Zeeman patterns arise from strong Paschen-Back interaction of the closely spaced even levels designated as $5097_{5 / 2}$, $5098_{7 / 2}, 5099_{9 / 2}$ and $5103_{3 / 2}, 5104_{5 / 2}, 5105_{1 / 2}$. Often even fully resolved, symmetrical Zeeman patterns of Re lines are not easy to interpret (apart from $J$-values) because the splitting factors $(g)$ usually depart more or less from Landé values. In fact these $g$-factors prove that besides configuration interaction the electron coupling in Re is neither $L S$ nor JJ, but usually intermediate; this will be discussed in connection with spectral term designations. A few of the levels in table 1 have two $g$-values owing to incomplete observations of the Zeeman patterns; in these cases the second possible value of $g$ is given in parentheses. Magnetic splitting factors, $g$, are now known for more than 75 percent of all known levels of Re I.

In table 1 the column headed "Origin" shows where levels previously known have been reported for the first time, according to the code explained at the end of that table. This gives a view of the progress from 1931 to 1956 in searching for atomic energy levels in the first spectrum of rhenium. Finally figure 2 gives a survey of the positions of presently known Re I energy levels grouped according to $J$-values.

\section{Spectral Lines of Re I}

Attempts to describe the spectra of rhenium, including the magnetic splitting (Zeeman effect), are plagued by relatively coarse hyperfine structure exhibited by most of the lines, many of which have 6 components (see fig. 1). This makes it difficult to estimate relative intensities and in some cases hyperfine structure is easily mistaken for fine structure. For lines that show flag patterns a mean wavelength was recorded, but when hyperfine components appear as two lines or groups of lines it is impossible to distinguish them from close fine structures. A good example of the latter are the two close levels 27384.40 and $27385.20 \mathrm{~K}$, both having $J=1 / 2$, which are retained in tables 1 and 2 as individual atomic energy levels although there is evidence that they are hyperfine levels. Another example is $44703.37_{5 / 2}$ whose combinations are 


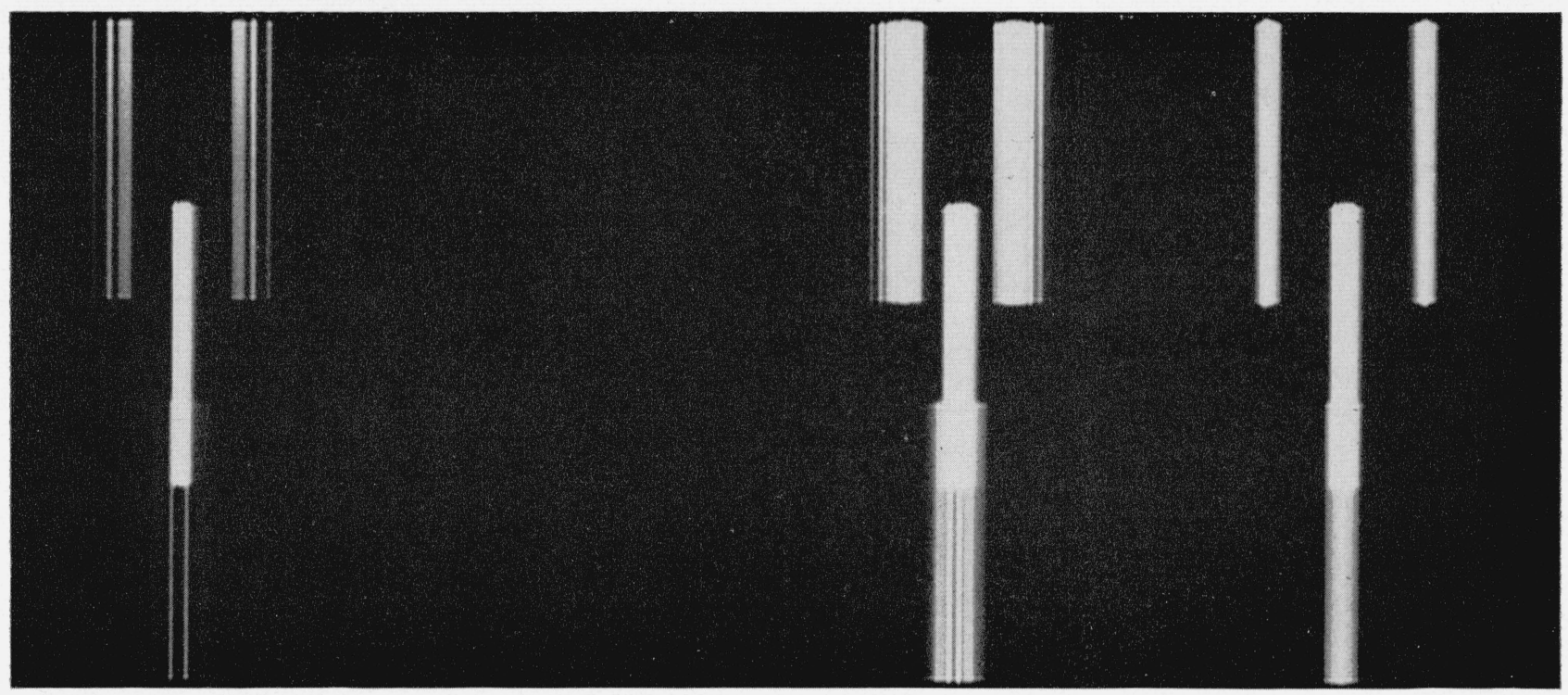

3451.9

3460.5

3464.7

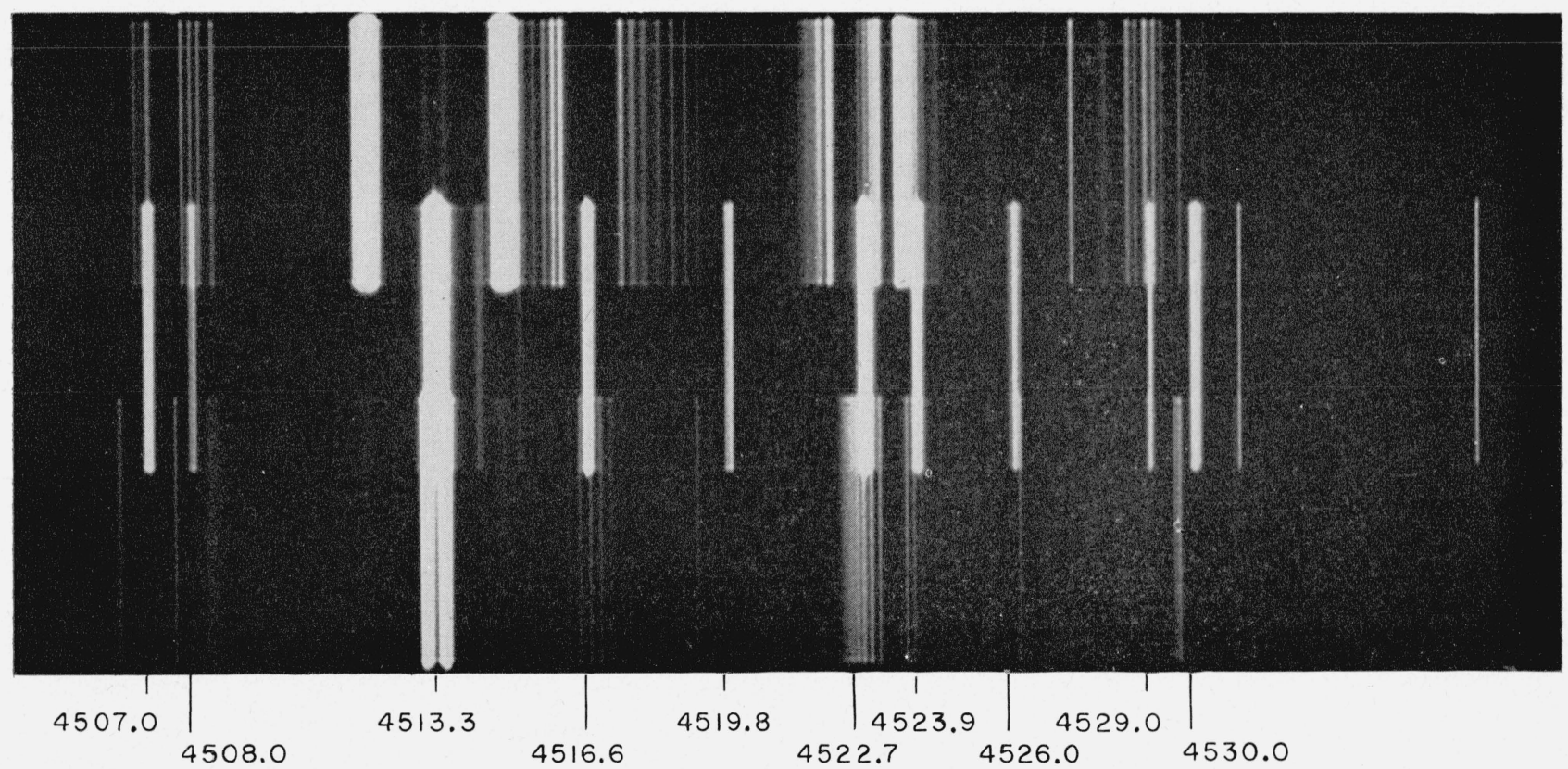

FiguRE 1. Zeeman patterns of Re I lines at 81,000 oersteds.

$\begin{array}{ll}\text { Upper: } & \sigma \text { components-perpendicular polarization. } \\ \text { Center: } & \text { Arc spectrum without magnetic field. } \\ \text { Lower: } & \pi \text { components-parallel polarization. } \\ \text { Numbers: Wavelengths of Re I lines in angstroms. }\end{array}$

usually observed as widened doublets which possibly represent perturbed or mutilated hyperfine multiplets (see note 28 at end of table 2 ). Several combinations of level 15165.89 $9_{1 / 2}$ are double $(4394,4417,4565 \mathrm{~A}$ ) but this seems to be caused by the involved odd levels, because most combinations of $151_{1 / 2}$ are single (see note 24 at end of table 2). As compensation for the complication introduced by hyperfine structure it may be said that the width and shading of flag patterns confirm this analysis qualitatively and have aided in the assignment of levels to electron configurations and term groups. Detailed proof of this statement is omitted in this paper which deals primarily with fine structure and therefore ignores hyperfine structure (excepting the complications mentioned above). Information about the resolution, width, and shading of flag patterns in rhenium spectra is given in Meggers' recent description of these spectra [8] which provided most of the material for this analysis. 
Even Odd

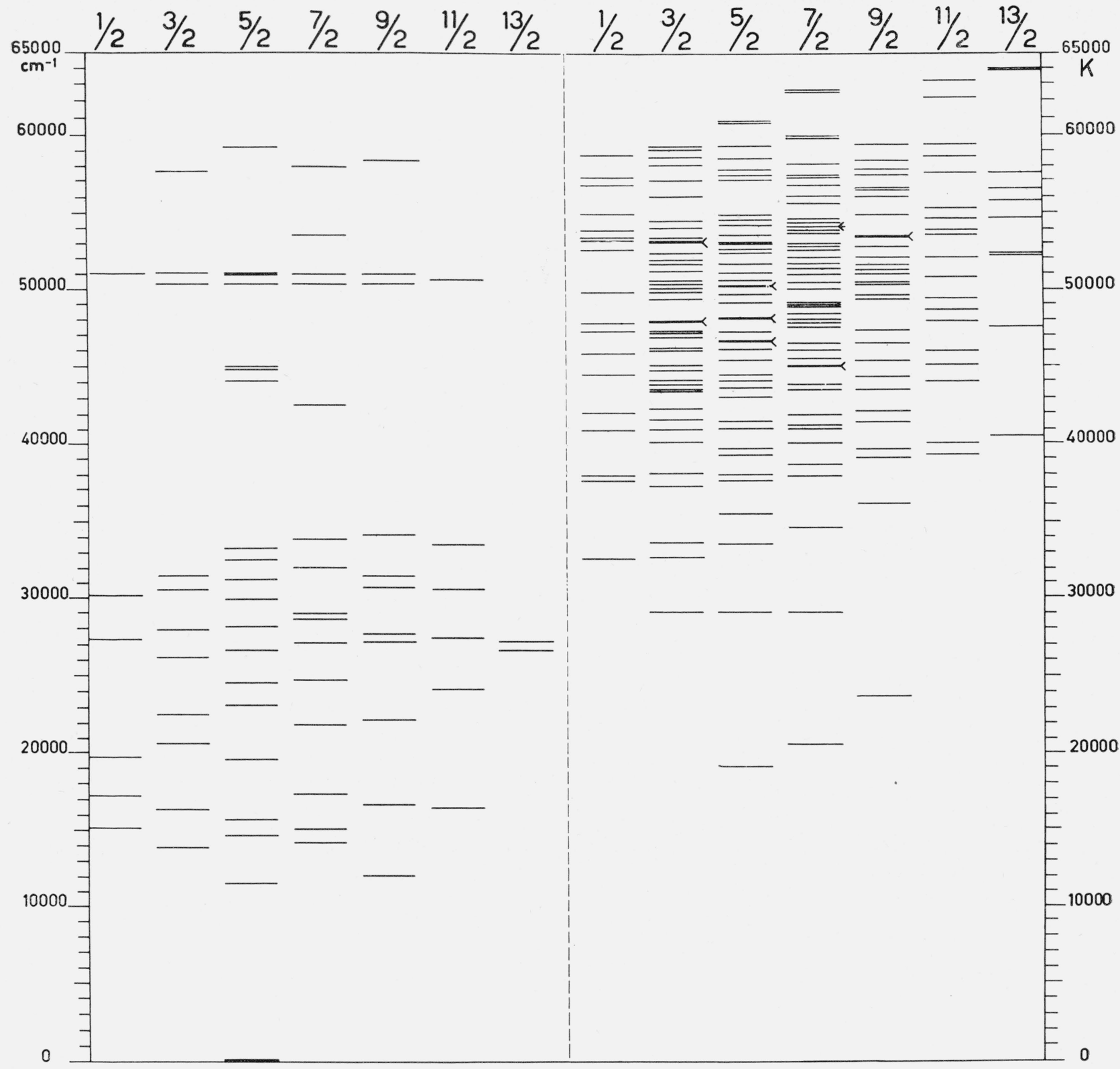

Figure 2. Energy-level diagram of Re $\mathrm{I}$.

Abscissae: $J$-values 蚫

Ordinates: kaysers, ${ }_{1}^{1} 1 \mathrm{~K}=1 \mathrm{~cm}^{-1}$

Table 2 contains all relevant data on the classified lines of Re I, including estimated relative intensities, measured wavelengths, derived vacuum wave numbers, observed Zeeman patterns, transitions between designated atomic energy levels, differences between wave numbers derived from measured wavelengths and those calculated from atomic energy levels, and notes that are appended to the table.

In table 2 , column 4 , the observed displacements of magnetic components are given in Lorentz units, the $\pi$ components being inclosed by parentheses and followed by the $\sigma$ components. When there are more than one component of either type the strongest is shown in bold face. Many unresolved Zeeman patterns are accompanied by letters A, B, C, or D which were adopted for conveniently indicating standard types according to Back and Landé [14]. The letter A means that the strongest $\pi$ components have the greatest displacement whereas the displacement of the strongest $\sigma$ components is the 
average of all. B means that the strongest $\pi$ components are least and the strongest $\sigma$ components most displaced. $\mathrm{C}$ indicates that the strongest $\pi$ and $\sigma$ are both least displaced. The letter D applies to relatively weak lines for which no $\sigma$ components were observed, so that it is impossible to distinguish between types B and C. A dagger ( $\dagger$ ) means that the pattern is unsymmetrical, and an asterisk (*) that the observed Zeeman pattern belongs to a Re II line. P-B indicates that a perturbation of Paschen-Back type has been observed.

The total number of classified lines of Re I in the region 1700 to $12000 \mathrm{~A}$ is now 2,764. Of these 116 are doubly and 5 are trebly classified, because the resolving power was inadequate to separate them. There are 6 cases where two observed lines have been classified as one transition, because the doubling is believed to be caused by perturbed hyperfine structures. Thus for some purposes the employed resolving power was too small and for others it was too large. Some of the complications that arose on account of hyperfine structure were mentioned in the preceding section.

A few lines classified as Re I were observed only on spark spectrograms measured in the Zeeman laboratory. Some of these have also been classified as $\mathrm{Re}$ II lines, and in these complex spectra it is certainly probable that occasionally $\operatorname{Re} \mathrm{I}$ and $\mathrm{Re}$ II lines coincide within the limits of spectrographic resolving power employed. In any case such lines appearing in table 2 represent permitted transitions between established energy levels of $\mathrm{Re} \mathrm{I}$ within reasonable limits of tolerance for these transitions.

Excepting lines of very short wavelengths, near $2000 \mathrm{~A}$ for which an error of $0.01 \mathrm{~A}$ corresponds to $0.25 \mathrm{~K}$, the difference between observed and calculated wave number ( $\mathrm{O}-\mathrm{C}$ in table 2) of classified $\mathrm{Re}$ I lines rarely exceeds $0.3 \mathrm{~K}$. For 563 lines in table 2 this difference is naught; for the remainder it is $\pm 0.06_{5} \mathrm{~K}$ and for the total number the average difference is $\pm 0.05 \mathrm{~K}$. Recalling that on account of hyperfine structure many Re lines have wid ths of the order of $2 \mathrm{~K}$, this small average departure of calculated from observed wave number of classified Re I must be regarded as satisfactory; it gives assurance that the mean wavelength of the wide lines is very near the actual center of gravity.

In the recent description of the arc spectrum of rhenium by Meggers [8] there are 4,143 lines that may be ascribed to Re I. Of these 2,660 are classified in this analysis (table 2). Although this number of classified Re I lines is only 64 percent of the total number it accounts for 90 percent of the total observed intensity. The remaining unclassified lines, with few exceptions, are of low intensity, between 40 and 1 . This is obvious from table 3 which lists all the unclassified Re I lines having intensity 50 or more. Only two of these are visible; the remainder are all in the ultraviolet short of $3000 \mathrm{~A}$. This suggests that some high energy levels of Re I are still unknown, and that further progress in the analysis of this spectrum might be made if an improved description in the extreme ultraviolet could be obtained.

\section{Interpretation of the Re I Levels}

Previous interpretations $[1,2,3,5,6,7]$ of certain Re I levels were made without the aid of Zeeman effect; they were guided by the simple interval and intensity rules of multiplet structure and by comparison with the known terms of Mn I. Now despite the large number of observed Zeeman patterns and computed $g$-factors, there are still only relatively few Re I levels that can be interpreted with certainty; 61 percent (171 out of 282) of the established levels must be characterized as "miscellaneous". However, compared with the earlier identifications, definite progress has resulted from the Zeeman-effect data despite many departures of $g$-factors from the Landé values associated with conventional spectral-term notation. We are pleased to report that most of the previous interpretations have been confirmed by the $g$-factors found, even those of $d^{5} s^{2}{ }^{4} \mathrm{P}$ which had been questioned [6]. The Re I levels, terms, and interpretations are displayed in table 4 .

Because manganese, technetium, and rhenium are homologous chemical elements their spectra are bound to show similarities. Indeed, such similarities, when first discovered in Re and Tc, were regarded as proof that these elements are really homologs of $\mathrm{Mn}$. In each case the spectra are highly complex because there are 7 optical electrons, and spectral terms of 4 different multiplicities (doublets, quartets, sextets, and octets) occur. Because the $d$-shell of electrons is usually half (or more) filled the terms are partly regular, partly inverted, and sometimes partially inverted. The principal differences between the three spectra, in general, are exhibited by larger term intervals for heavier atoms, and by the relative stability of various configurations of electrons.

In general, the structure of the low even system of $\mathrm{Re} \mathrm{I}$ is comparable to that of $\mathrm{Mn} \mathrm{I} \mathrm{[15].} \mathrm{The}$ relative positions of the Re I lowest terms from $d^{5} s^{2}$ and $d^{6} s$ are very similar to those of $\mathrm{Mn} \mathrm{I}$, and quite different from those of Tc I [16]. However, in Re I the terms from $d^{5} s^{2}$ are somewhat better known than in Mn I. This is due partly to the fact that intersystem combinations are much stronger in $\operatorname{Re} I$ because of departures from $L S$ coupling, so that many doublet levels could be found in Re I that were unknown in Mn I. The second reason is that the mutual electrostatic interactions in $R$ e I are rather different from those in Mn I. This is immediately obvious from the fact that in the $d^{5} s^{2}$ configuration ${ }^{4} \mathrm{P}$ is below ${ }^{4} \mathrm{G}$ in Re $\mathrm{I}$, whereas the opposite obtains in Mn I. When taking this into account one finds from theoretical consideration [17] that the doublet terms should be depressed with respect to the quartets in Re I as compared with Mn I. An analogous situation exists in the spectra of $W$ I versus Cr I.

The same $d^{6} s$ terms are found in Re I and $\mathrm{Mn}$ I excepting two doublets above $38000 \mathrm{~K}$ in the latter. The new 13/2 level 270 in Re I has much stronger combinations than the previously found $13 / 2$ level 263 that was interpreted [5] as ${ }^{4} \mathrm{H}$. This, and the fact that the new level had a $g$-value much nearer to the Landé value for ${ }^{4} \mathrm{H}_{13 / 2}$ led us to identify level 
270 as $\left(5 d^{6} 6 s\right)^{4} \mathrm{H}_{13 / 2}$. This is certainly the most plausible interpretation because there are only three $13 / 2$ levels anticipated in the low even system, namely $d^{5} s^{2}{ }^{2} \mathrm{I}_{13 / 2}, \quad d^{6} s^{4} \mathrm{H}_{13 / 2}$, and $d^{6} s^{2} \mathrm{I}_{13 / 2}$. The lower level 263 is readily explained as the first ${ }^{2} \mathrm{I}$ whereas the other ${ }^{2} \mathrm{I}$ presumably lies too high to be detected. In view of recent progress with the analysis of $\mathrm{Mn}$ I [15] the earlier interpretation [5] of four Re I levels above $22000 \mathrm{~K}$ as $5 d^{7} \mathrm{~F}$ cannot be maintained; it is concluded that no $d^{7}$ levels have been found in Re I. In fact all the known low even levels of Re I have been assigned to $d^{5} s^{2}$ or $d^{6} s$. Besides the identifications mentioned above, additional assignments of conventional term symbols have been suggested by R. E. Trees, who calculated approximately the relative positions of known and anticipated spectral terms; these calculations will be reported in a separate paper by Trees.

A brief discussion of the Re I level $273_{1 / 2}$ seems justified because of the trouble caused by hyperfine structure. At first two levels were detected at nearly the same value, viz., 27384.40 and 27385.20 K. Generally these combined with the same odd levels, thus yielding 10 pairs of very close lines of comparable intensities. In three more cases only a somewhat broadened line was observed with a wave number intermediate between the calculated values. In some other cases only the transition to $273^{\prime}$ or $273^{\prime \prime}$ was present and because $273^{\prime \prime}$ had two combinations with odd $5 / 2$ levels while $273^{\prime}$ had none, the latter was regarded as a $1 / 2$ level and the former as a $3 / 2$, with the possible interpretations $d^{6} s^{4} \mathrm{P}_{1 / 2}$ and $d^{5} s^{2}{ }^{4} \mathrm{D}_{3 / 2}$. All the lines involved were relatively weak. For $273^{\prime \prime}$ no Zeeman-effect measurements were available, but for $273^{\prime}$ there was a measurement of two $\pi$ components of $4369.64 \mathrm{~A}$ $\left(273^{\prime}{ }_{1 / 2}-5026_{3 / 2}\right)$. This splitting pointed to a $g$-value of either 2.396 or 0.072 , the first being compatible with the interpretation of $273^{\prime}$ as ${ }^{4} \mathrm{P}_{1 / 2}$ and the second with ${ }^{4} \mathrm{D}_{1 / 2}$. Upon remeasurement of the Zeeman spectrogram the two $\pi$ components were found to be symmetrical around the wavelength $4369.70 \mathrm{~A}$, just the average of the wavelengths 4369.64 and $4369.77 \mathrm{~A}$ reported [8] for Re I. Hence we must conclude that these lines are hyperfine structure components of one and the same transition and that $273^{\prime}$ and $273^{\prime \prime}$ are hyperfine structure sublevels of one electronic level having $J=1 / 2$. In tables 1 and 2 , it was found practical to retain the notation $273^{\prime}$ and $273^{\prime \prime}$, but in table 4 a single level $27384.80 \mathrm{~K}$ is interpreted as $d^{6} s{ }^{4} \mathbf{D}_{1 / 2}$.

The departures of the observed $g$-factors from their Landé values are often very large but in opposite directions, so that the $g$-sums are not very irregular even when some levels of the same $J$-value are not known. There are a few instances of $g$-sharing which suggest a strong mutual perturbation of two levels with exclusion of the rest. The most conspicuous example is $d^{5} s^{2}{ }^{4} \mathrm{G}_{7 / 2}$ (with $\Delta g=+0.17$ ) and $d^{6} s{ }^{4} \mathrm{D}_{7 / 2}$ (with $\Delta g=-0.17$ ). Furthermore, we have the two levels $d^{6} s^{6} \mathrm{D}_{1 / 2} \quad(\Delta g=-0.81)$ and $\left(d^{6} s\right)^{4} \mathrm{D}_{1 / 2}(\Delta g=+0.98)$. Also two levels with $J=$ $13 / 2$ have opposite but equal $g$-departures $\left(d^{5} s^{2}{ }^{2} \mathrm{I}_{13 / 2}\right.$ has $\Delta g=+0.02$ and $d^{6} s{ }^{4} \mathrm{H}_{13 / 2}$ has $\left.\Delta g=-0.02\right)$, so that the remaining unknown $13 / 2$ level $\left(d^{6} s^{2} \mathrm{I}_{13 / 2}\right)$ should have a normal $g$-factor. Attention was called [11] to large negative deviations of $g$-values for Re I terms that converge to $d^{5} s{ }^{5} \mathrm{~S}_{2}$ in Re II, which lacks 0.29 Lorentz unit from Landé value for that term. The present data are insufficient to test the sum rule for $g$-values of any configuration in Re $\mathrm{I}$.

The first identifications in the low even system served as a basis for the interpretation of a number of odd levels by means of the observed intensities of the combinations. When $g$-values became available these identifications could be extended, and verified by comparison with the parent structure of Re II. For example the low ${ }^{6} \mathrm{G}^{\circ}$ from $d^{5} s\left({ }^{5} \mathrm{G}\right) 6 p$ of Re I was not expected where found, but it is confirmed by the low ${ }^{5} \mathrm{G}$ from $d^{5} s$ found in Re II by Meggers, Catalán, and Sales [18].

From comparison of the transitions $d^{4} s^{6} \mathrm{D}_{9 / 2}-$ $d^{4} p^{6} \mathrm{~F}^{\circ}{ }_{11 / 2}$ in $\mathrm{V} \mathrm{I}, \mathrm{Nb} \mathrm{I}$, and $\mathrm{Ta} \mathrm{I}$, and $\left(d^{6} s\right)^{6} \mathrm{D}_{9 / 2}^{9 / 2}$ $d^{6} p^{6} \mathrm{~F}^{\circ}{ }_{11 / 2}$ in $\mathrm{Mn}$ I and Te I, it could be inferred that the corresponding transition in $\mathrm{Re}$ I should have a wave number of about $35000 \mathrm{~K}$. In agreement herewith $d^{6} p^{6} \mathrm{~F}^{\circ}{ }_{11 / 2}$ has been located in Re I at $45082.63 \mathrm{~K}$, and the line in question at $33328.06 \mathrm{~K}$. Beyond completing this ${ }^{6} \mathrm{~F}^{\circ}$ term no further identifications in the $d^{6} p$ configuration of Re 1 have been attempted. Excepting $d^{5} s p^{8} \mathrm{P}^{\circ}$ and ${ }^{6} \mathrm{P}^{\circ}$ the low odd terms of Re I belong to $d^{4} s^{2} p$. The term. ${ }^{6} \mathrm{D}$ and ${ }^{6} \mathrm{~F}$ derived from the parent term $d^{4} s^{2}{ }^{5} \mathrm{D}$ of Re II (which is about 14000 $\mathrm{K}$ above the ground state $\left.d^{5}\left({ }^{6} \mathrm{~S}\right) s^{7} \mathrm{~S}\right)$ have been identified. In accordance with this assignment the levels with small $J$-values are the lower ones, whereas the terms derived from $d^{6}{ }^{5} \mathrm{D}$ should be inverted, as they are.

Seventeen high even levels of Re I could readily be interpreted in connection with the known low Re II levels [18]. The previous interpretation [3] of level $5097_{3 / 2}$ as $d^{5} s\left({ }^{7} \mathrm{~S}\right) d{ }^{6} \mathrm{D}$ has been abandoned. The levels $5103_{3 / 2}$ and $5104_{5 / 2}$ turned out to belong to one multiple term because they show partial PaschenBack effects of transitions to the same odd level. The grouping of nearby levels with proper $J$-values led to recognition of the partially inverted term designated $e^{6} \mathrm{D}$. Both $e^{6} \mathrm{D}$ and $e^{8} \mathrm{D}$ have small intervals because their series limit is a ${ }^{7} \mathrm{~S}$ in Re II, and the available $g$-factors confirm the designations. The former interpretation [5] of level $5766_{3 / 2}$ was changed because the lower ${ }^{5} \mathrm{D}$ in Re II was assigned [18] to $d^{4} s^{2}$ instead of $d^{6}$. In the course of this analysis of Re I we succeeded in supplementing this $3 / 2$ level with $5 / 2,7 / 2$, and $9 / 2$ to form the term designated as $f^{6} \mathrm{D}$, with only the $1 / 2$ level still lacking. These interpretations are supported by the observed intensities of the transitions to the odd levels which are displayed in figure 3 , where the observed intensity is roughly represented by the size of the black circle, and shaded circles indicate outstanding intensities. The most striking feature of figure 3 is that the levels of $d^{5} s\left({ }^{5} \mathrm{D}\right) s{ }^{6} \mathrm{D}$ combine much more strongly with the higher odd ${ }^{6} \mathrm{D}$ than with the lower one. The explanation is that the former transitions (to $d^{5}$ $\left.s\left({ }^{5} \mathrm{P}\right) p^{6} \mathrm{D}\right)$ require only one electron jump ( $p$ to $\left.s\right)$, whereas the latter transitions (to $d^{4} s^{2}\left({ }^{5} \mathrm{D}\right) p{ }^{6} \mathrm{D}$ ) 


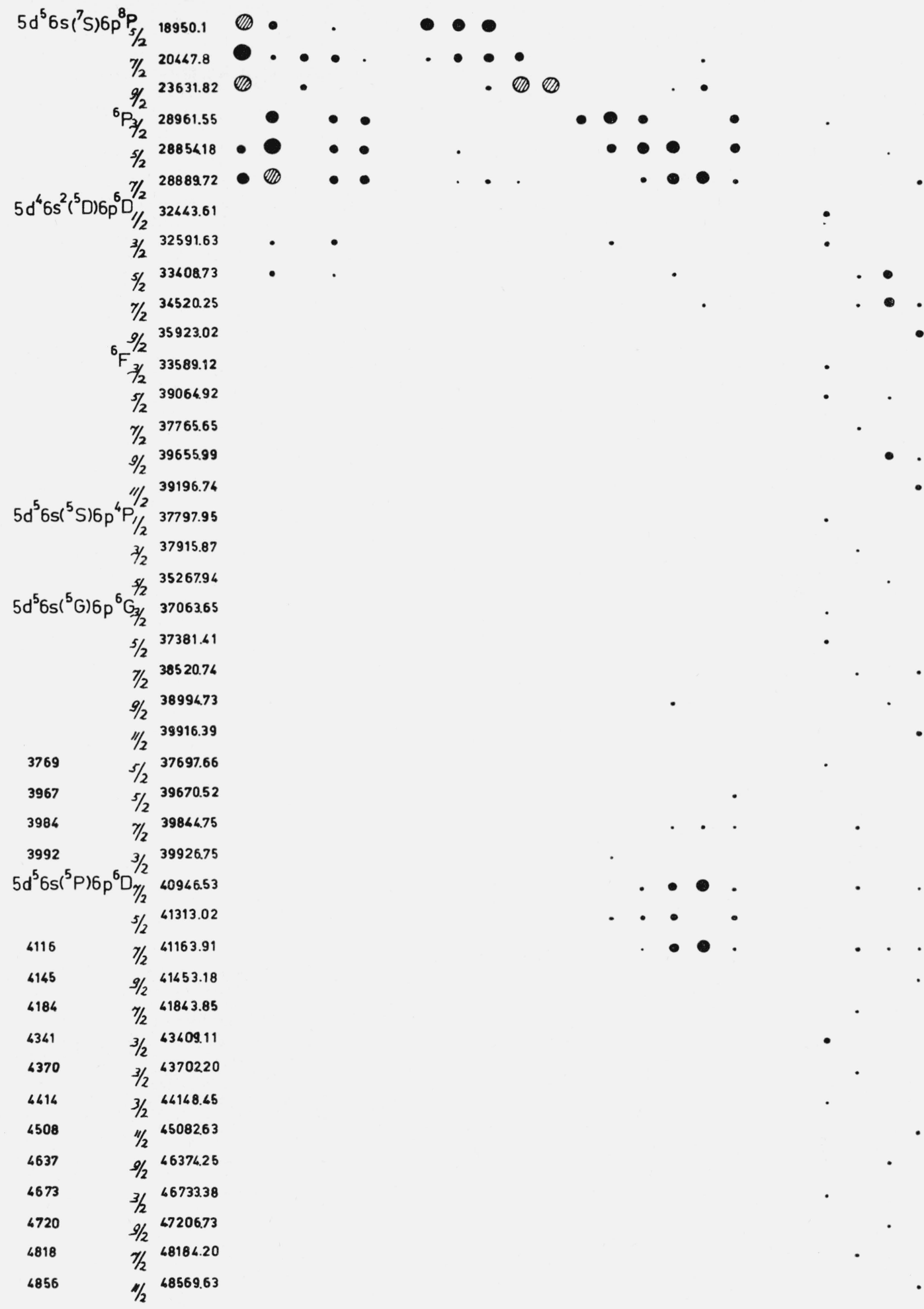

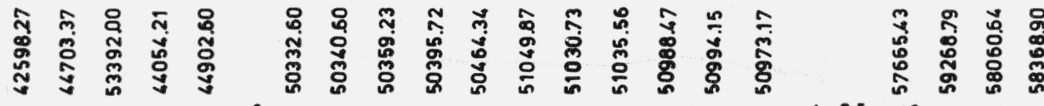

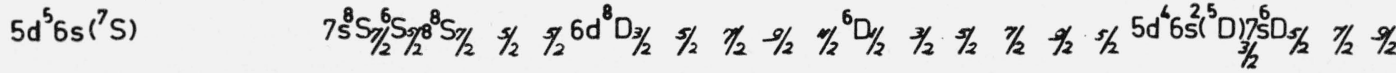
FIGURE 3. Line intensities of transition; between Re I levels. 
involve a simultaneous jump of two electrons ( $p$ to $s$ and $s$ to $d$ ).

In the high even group of Re I there are two $5 / 2$ levels the parent terms of which should be very low in Re II, but are still unknown. The Re I levels in question have values 44054.21 and $44902.60 \mathrm{~K}$; they are well established (see fig. 3) but without $g$-factors. These levels suggest the existence of $R_{e}$ II levels between 0 and $13777 \mathrm{~K}$, for which supposition there is no further evidence except that $d^{6}{ }^{5} \mathrm{D}$ is still unknown. Another unidentified high even level in Re $\mathrm{I}$ is at $50973.17 \mathrm{~K}$, but in this energy range there are more possibilities, and this lone level is left as an invitation to further analysis and interpretation.

In his first paper Meggers [1] derived from two ${ }^{8} \mathrm{~S}$ terms in series a spectroscopic convergence limit of $63530 \mathrm{~K}$ for Re I. In his second paper Klinkenberg [5] derived from two ${ }^{6} \mathrm{~S}$ terms a Rydberg-series limit of $65560 \mathrm{~K}$, which he regarded as an upper limit because the proper correction is unknown. If the Rydberg denominator deviates only 0.04 from. unity this limit would be reduced to $63000 \mathrm{~K}$. Because there is at present no further information on spectral series in Re I the original limit is accepted, and the ionization potential remains near 7.87 electron volts.

The present description and interpretation of Re I has been a long and difficult task; it is still far from satisfactory or complete but may require additional experimental and theoretical study before it can be continued. This analysis has been pushed to its present state primarily so that table 4 could be reproduced in Volume III of Atomic Energy Levels [19], where detailed comparisons with other tables may give inspiration for further progress in the quantum interpretation of complex atomic spectra.

\section{References}

[1] W. F. Meggers, BS J. Research 6, 1027 (1931) RP322.

[2] W. F. Meggers, BS J. Research 10, 757 (1933) RP564.

[3] P. F. A. Klinkenberg, Physica 13, 581 (1947).

[4] G. R. Harrison, MIT Wavelength Tables (John Wiley \& Sons, Inc., New York, N. Y., 1939).

[5] P. F. A. Klinkenberg, Physica 14, 269 (1948).

[6] R. Velasco, Anales real soc. espan. fis. y quim. [A] 45, 215 (1949).

[7] R. Velasco, Anales real soc. espan. fis. y quim. [A] 48, 55 (1952).

[8] W. F. Meggers, J. Research NBS, 49, 187 (1952) RP2355.

[9] G. R. Harrison and F. Bitter, Phys. Rev. 5y, 15 (1940).

[10] G. R. Harrison, Repts. Progr. in Phys. 8, 212 (1941).

[11] W. F. Meggers, M. A. Catalán, and R. Velasco, J. Opt. Soc. Am. 42, 288 (1952).

[12] B. Edlén, J. Opt. Soc. Am. 43, 339 (1953)

[13] Transactions of the Joint Commission for Spectroscopy, J. Opt. Soc. Am. 43, 412 (1953).

[14] E. Back and A. Landé, Zeeman effect und Multipletstruktur der Spektrallinien, 168 (Springer, Berlin, 1925).

[15] C. E. Moore, Atomic Energy Levels II, 27-32, NBS Circ. 467 (1952).

[16] W. F. Meggers, J. Research NBS 47, 7 (1951) RP2221.

[17] O. Laporte and J. R. Platt, Phys. Rev. 61, 305 (1942).

[18] W. F. Meggers, M. A. Catalán, and M. Sales (in press).

[19] C. E. Moore, Atomic Energy Levels, III, NBS Circ. 467 (in press).

TABLE 1. Energy levels of the Re atom

\begin{tabular}{|c|c|c|c|c|c|c|c|c|c|}
\hline $\begin{array}{l}\text { Desig- } \\
\text { nation }\end{array}$ & Value & $J$ & $g$ & Origin & $\begin{array}{l}\text { Desig- } \\
\text { nation }\end{array}$ & Value & $J$ & $g$ & Origin \\
\hline \multicolumn{10}{|c|}{ LOW EVEN GROUP } \\
\hline $\begin{array}{r}0 \\
115 \\
117 \\
138 \\
142\end{array}$ & $\begin{array}{r}0.00 \\
11583.96 \\
11754.52 \\
13826.12 \\
14216.86\end{array}$ & $\begin{array}{l}5 / 2 \\
5 / 2 \\
9 / 2 \\
3 / 2 \\
7 / 2\end{array}$ & $\begin{array}{l}\text { 1. } 950 \\
\text { 1. } 278 \\
\text { 1. } 545 \\
\text { 1. } 485 \\
\text { 1. } 567\end{array}$ & $\begin{array}{ll}\text { M } & 31 \\
\text { M } & 31 \\
\text { M } & 31 \\
\text { M } & 31 \\
\text { M } & 31\end{array}$ & $\begin{array}{l}266 \\
270 \\
271 \\
272 \\
273\end{array}$ & $\begin{array}{l}26661.43 \\
27130.14 \\
27141.13 \\
27161.35 \\
27243.88\end{array}$ & $\begin{array}{r}5 / 2 \\
13 / 2 \\
7 / 2 \\
9 / 2 \\
11 / 2\end{array}$ & $\begin{array}{l}\text { 1. } 32 \\
\text { 1. } 20_{8} \\
\text { 1. } 34 \\
\text { 1. } 18\end{array}$ & $\begin{array}{ll}\mathrm{K} & 47 \\
& \\
\mathrm{M} & 33 \\
\mathrm{~K} & 47 \\
\mathrm{~K} & 48\end{array}$ \\
\hline $\begin{array}{l}146 \\
150 \\
151 \\
157 \\
163\end{array}$ & $\begin{array}{l}14621.46 \\
15058.19 \\
15165.89 \\
15770.42 \\
16307.15\end{array}$ & $\begin{array}{r}5 / 2 \\
7 / 2 \\
1 / 2 \\
5 / 2 \\
11 / 2\end{array}$ & $\begin{array}{l}\text { 1. } 151 \\
\text { 1. } 153 \\
\text { 2. } 368 \\
\text { 1. } 309 \\
\text { 1. } 242\end{array}$ & $\begin{array}{ll}\text { M } & 31 \\
\text { M } & 31 \\
\text { K } & 47 \\
\text { M } & 31 \\
\text { M } & 31\end{array}$ & $\begin{array}{l}273^{\prime} \\
273^{\prime \prime} \\
275 \\
278 \\
280\end{array}$ & $\begin{array}{l}27384.40 \\
27385.20 \\
27514.31 \\
27827.65 \\
28030.32\end{array}$ & $\begin{array}{l}1 / 2 \\
1 / 2 \\
9 / 2 \\
3 / 2 \\
5 / 2\end{array}$ & $\begin{array}{l}2.396(0.072) \\
\text { 1. } 13 \\
0.888 \\
1.12\end{array}$ & $\begin{array}{ll} & \\
\text { K } & 47 \\
\text { K } & 48\end{array}$ \\
\hline $\begin{array}{l}164 \\
166 \\
172 \\
173 \\
194\end{array}$ & $\begin{array}{l}16327.51 \\
16619.28 \\
17238.30 \\
17330.82 \\
19457.89\end{array}$ & $\begin{array}{l}3 / 2 \\
9 / 2 \\
1 / 2 \\
7 / 2 \\
5 / 2\end{array}$ & $\begin{array}{l}\text { 1. } 706 \\
\text { 1. } 175 \\
\text { 2. } 521 \\
\text { 1. } 255 \\
\text { 1. } 361\end{array}$ & $\begin{array}{ll}\text { M } & 31 \\
\text { M } & 31 \\
\text { M } & 31 \\
\text { M } & 31 \\
\text { M } & 33\end{array}$ & $\begin{array}{l}285 \\
288 \\
298 \\
301 \\
304\end{array}$ & $\begin{array}{l}28542.13 \\
28809.87 \\
29800.38 \\
30131.57 \\
30526.60\end{array}$ & $\begin{array}{l}7 / 2 \\
7 / 2 \\
5 / 2 \\
1 / 2 \\
3 / 2\end{array}$ & $\begin{array}{r}0.93(1.44) \\
\sim 1.17\end{array}$ & $\begin{array}{ll}\text { K } & 48 \\
-\bar{K} & 48 \\
\text { K } & 48\end{array}$ \\
\hline $\begin{array}{l}197 \\
204 \\
217 \\
221 \\
224\end{array}$ & $\begin{array}{l}\text { 19757. } 91 \\
20481.73 \\
21775.40 \\
22160.04 \\
22422.83\end{array}$ & $\begin{array}{l}1 / 2 \\
3 / 2 \\
7 / 2 \\
9 / 2 \\
3 / 2\end{array}$ & $\begin{array}{l}0.983 \\
\text { 1. } 451 \\
\text { 1. } 135 \\
\text { 1. } 198 \\
0.781\end{array}$ & $\begin{array}{ll}\mathrm{K} & 47 \\
\mathrm{~K} & 47 \\
\mathrm{M} & 31 \\
\mathrm{~K} & 47 \\
\mathrm{~K} & 47\end{array}$ & $\begin{array}{l}305 \\
306 \\
311 \\
313 \\
314\end{array}$ & $\begin{array}{l}30559.91 \\
30645.33 \\
31186.08 \\
31399.30 \\
31460.62\end{array}$ & $\begin{array}{r}11 / 2 \\
9 / 2 \\
5 / 2 \\
9 / 2 \\
3 / 2\end{array}$ & \begin{tabular}{l} 
1. 07 \\
1. $17 ?$ \\
\hdashline$-\ldots$
\end{tabular} & 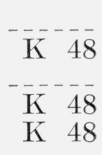 \\
\hline $\begin{array}{l}231 \\
239 \\
244 \\
247 \\
261\end{array}$ & $\begin{array}{l}23154.81 \\
23956.00 \\
24425.40 \\
24724.22 \\
26131.57\end{array}$ & $\begin{array}{r}5 / 2 \\
11 / 2 \\
5 / 2 \\
7 / 2 \\
3 / 2\end{array}$ & $\begin{array}{l}\text { 1. } 189 \\
0.995 \\
\text { 1. } 067 \\
\text { 1. } 03 \\
0.650\end{array}$ & $\begin{array}{ll}\text { M } & 33 \\
\mathrm{~K} & 47 \\
\mathrm{~K} & 47 \\
\mathrm{M} & 33 \\
\mathrm{M} & 33\end{array}$ & $\begin{array}{l}319 \\
324 \\
332 \\
333 \\
338\end{array}$ & $\begin{array}{l}31982.99 \\
32435.14 \\
33281.65 \\
33317.57 \\
33823.66\end{array}$ & $\begin{array}{r}7 / 2 \\
5 / 2 \\
5 / 2 \\
11 / 2 \\
7 / 2\end{array}$ & 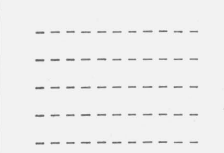 & $\begin{array}{l}\text { K } 48 \\
-\cdots \\
-\cdots-1\end{array}$ \\
\hline 263 & 26348. 96 & $13 / 2$ & 1. 100 & K 48 & 341 & 34194. 27 & $9 / 2$ & $----1--1-$ & ----- \\
\hline
\end{tabular}


TABLE 1. Energy levels of the Re atom-Continued

\begin{tabular}{|c|c|c|c|c|c|c|c|c|c|}
\hline $\begin{array}{l}\text { Desig- } \\
\text { nation }\end{array}$ & Value & $J$ & $g$ & Origin & $\begin{array}{l}\text { Desig- } \\
\text { nation }\end{array}$ & Value & $J$ & $g$ & Origin \\
\hline \multicolumn{10}{|c|}{ HIGH EVEN GROUP } \\
\hline $\begin{array}{l}4259 \\
4405 \\
4470 \\
4491 \\
5033\end{array}$ & $\begin{array}{l}42598.27 \\
44054.21 \\
44703.37 \\
44902.60 \\
50332.60\end{array}$ & $\begin{array}{l}7 / 2 \\
5 / 2 \\
5 / 2 \\
5 / 2 \\
3 / 2\end{array}$ & \begin{tabular}{l} 
1. 957 \\
1. 866 \\
\hdashline-
\end{tabular} & $\begin{array}{ll}\text { M } & 31 \\
\text { M } & 31 \\
\text { M } & 31 \\
\text { M } & 31 \\
\text { M } & 31\end{array}$ & $\begin{array}{l}5098 \\
5099 \\
5103 \\
5104 \\
5105\end{array}$ & $\begin{array}{l}50988.47 \\
50994.15 \\
51030.73 \\
51035.56 \\
51049.87\end{array}$ & $\begin{array}{l}7 / 2 \\
9 / 2 \\
3 / 2 \\
5 / 2 \\
1 / 2\end{array}$ & $\begin{array}{l}\text { 1. } 558 \\
1.548 \\
1.546 \\
\end{array}$ & $\begin{array}{ll}\text { M } & 31 \\
\text { M } & 31 \\
\text { M } & 31 \\
\text { M } & 31 \\
\text { C } & 53\end{array}$ \\
\hline $\begin{array}{l}5034 \\
5035 \\
5039 \\
5047 \\
5097 \\
\end{array}$ & $\begin{array}{l}50340.62 \\
50359.23 \\
50395.72 \\
50464.34 \\
50973.17 \\
\end{array}$ & $\begin{array}{r}5 / 2 \\
7 / 2 \\
9 / 2 \\
11 / 2 \\
5 / 2 \\
\end{array}$ & $\begin{array}{l}\text { 2. } 027 \\
\text { 1. } 820 \\
\text { 1. } 690 \\
\text { 1. } 629 \\
\text { 1. } 489\end{array}$ & $\begin{array}{ll}\text { M } & 31 \\
\text { M } & 31 \\
\text { M } & 31 \\
\text { M } & 31 \\
\text { M } & 31\end{array}$ & $\begin{array}{l}5339 \\
5766 \\
5806 \\
5836 \\
5926 \\
\end{array}$ & $\begin{array}{l}53392.00 \\
57665.43 \\
58060.64 \\
58368.90 \\
59268.79 \\
\end{array}$ & $\begin{array}{l}7 / 2 \\
3 / 2 \\
7 / 2 \\
9 / 2 \\
5 / 2 \\
\end{array}$ & 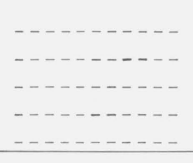 & \begin{tabular}{c} 
M 31 \\
K 48 \\
\hdashline$-\ldots-$ \\
$-\ldots$
\end{tabular} \\
\hline \multicolumn{10}{|c|}{ ODD GROUP } \\
\hline $\begin{array}{l}1895 \\
2044 \\
2363 \\
2885 \\
2888\end{array}$ & $\begin{array}{l}18950.1 \\
20447.8 \\
23631.82 \\
28854.18 \\
28889.72\end{array}$ & $\begin{array}{l}5 / 2 \\
7 / 2 \\
9 / 2 \\
5 / 2 \\
7 / 2\end{array}$ & $\begin{array}{l}\text { 2. } 274 \\
\text { 1. } 926 \\
\text { 1. } 768 \\
\text { 1. } 871 \\
\text { 1. } 709\end{array}$ & $\begin{array}{ll}\text { M } & 31 \\
\text { M } & 31 \\
\text { M } & 31 \\
\text { M } & 31 \\
\text { M } & 31\end{array}$ & $\begin{array}{l}4394 \\
4399 \\
4414 \\
4422 \\
4430\end{array}$ & $\begin{array}{l}43949.98 \\
43996.30 \\
44148.45 \\
44224.58 \\
44308.73\end{array}$ & $\begin{array}{r}5 / 2 \\
11 / 2 \\
3 / 2 \\
9 / 2 \\
5 / 2\end{array}$ & $\begin{array}{l}\text { 1. } 385 \\
\text { 1. } 26 \\
\text { 1. } 573 \\
\text { 1. } 244 \\
\text { 1. } 223\end{array}$ & $\begin{array}{ll}\mathrm{K} & 47 \\
\mathrm{~K} & 47 \\
\mathrm{~K} & 47 \\
\mathrm{M} & 31 \\
\mathrm{M} & 31\end{array}$ \\
\hline $\begin{array}{l}2896 \\
3244 \\
3259 \\
3340 \\
3358\end{array}$ & $\begin{array}{l}28961.55 \\
32443.61 \\
32591.63 \\
33408.73 \\
33589.12\end{array}$ & $\begin{array}{l}3 / 2 \\
1 / 2 \\
3 / 2 \\
5 / 2 \\
3 / 2\end{array}$ & $\begin{array}{l}\text { 2. } 333 \\
1.762 \\
1.500 \\
2.335\end{array}$ & $\begin{array}{ll}\text { M } & 31 \\
\text { M } & 31 \\
\text { M } & 31 \\
\text { M } & 31 \\
\text { M } & 31\end{array}$ & $\begin{array}{l}4441 \\
4472 \\
4490 \\
4494 \\
4508\end{array}$ & $\begin{array}{l}44416.33 \\
44720.09 \\
44901.15 \\
44946.12 \\
45082.63\end{array}$ & $\begin{array}{r}1 / 2 \\
3 / 2 \\
7 / 2 \\
7 / 2 \\
11 / 2\end{array}$ & $\begin{array}{l}0.15 \\
1.330 \\
1.253 \\
0.905 \\
1.40\end{array}$ & $\begin{array}{ll}\mathrm{K} & 47 \\
\mathrm{~K} & 47 \\
\mathrm{M} & 31 \\
\mathrm{~K} & 47 \\
\mathrm{M} & 31\end{array}$ \\
\hline $\begin{array}{l}3452 \\
3526 \\
3592 \\
3706 \\
3738\end{array}$ & $\begin{array}{l}34520.25 \\
3526 \% .94 \\
35923.02 \\
37063.65 \\
37381.41\end{array}$ & $\begin{array}{l}7 / 2 \\
5 / 2 \\
9 / 2 \\
3 / 2 \\
5 / 2\end{array}$ & $\begin{array}{l}\text { 1. } 454 \\
\text { 1. } 385 \\
\text { 1. } 440 \\
0.626 \\
0.990\end{array}$ & $\begin{array}{ll}\text { M } & 31 \\
\text { M } & 31 \\
\text { M } & 31 \\
\text { M } & 31 \\
\text { M } & 31\end{array}$ & $\begin{array}{l}4512 \\
4533 \\
4534 \\
4546 \\
4581\end{array}$ & $\begin{array}{l}45121.81 \\
45332.03 \\
45343.57 \\
45462.83 \\
4581 \% .26\end{array}$ & $\begin{array}{l}3 / 2 \\
5 / 2 \\
9 / 2 \\
7 / 2 \\
1 / 2\end{array}$ & $\begin{array}{l}0.674 \\
1.088 \\
\text { 1. } 131 \\
\text { 1. } 300 \\
\text { 2. } 197\end{array}$ & $\begin{array}{ll}\mathrm{K} & 47 \\
\mathrm{~K} & 48 \\
\mathrm{M} & 31 \\
\mathrm{M} & 31\end{array}$ \\
\hline $\begin{array}{l}3769 \\
3776 \\
3779 \\
3791 \\
3852\end{array}$ & $\begin{array}{l}3769 \% .66 \\
37765.65 \\
37797.95 \\
37915.87 \\
38520.74\end{array}$ & $\begin{array}{l}5 / 2 \\
7 / 2 \\
1 / 2 \\
3 / 2 \\
7 / 2\end{array}$ & $\begin{array}{l}\text { 1. } 219 \\
\text { 1. } 335 \\
\text { 2. } 620 \\
\text { 1. } 495 \\
\text { 1. } 216\end{array}$ & $\begin{array}{c}\text { M } 31 \\
\text { M } 31 \\
-\bar{M} 31 \\
\text { M } 31\end{array}$ & $\begin{array}{l}4587 \\
4590 \\
4593 \\
4611 \\
4614\end{array}$ & $\begin{array}{l}45876.34 \\
45904.25 \\
4593 \% .18 \\
46112.24 \\
46141.11\end{array}$ & $\begin{array}{r}3 / 2 \\
11 / 2 \\
7 / 2 \\
5 / 2 \\
1 / 2\end{array}$ & $\begin{array}{l}\text { 1. } 384 \\
\text { 1. } 175 \\
\text { 1. } 298 \\
\text { 1. } 405 \\
0.716\end{array}$ & $\begin{array}{ll}\mathrm{K} & 47 \\
\mathrm{~K} & 47 \\
\mathrm{M} & 31 \\
\mathrm{M} & 31 \\
\mathrm{M} & 31\end{array}$ \\
\hline $\begin{array}{l}3899 \\
3906 \\
3919 \\
3965 \\
3967\end{array}$ & $\begin{array}{l}38994.78 \\
39064.92 \\
39196.74 \\
39655.99 \\
39670.52\end{array}$ & $\begin{array}{r}9 / 2 \\
5 / 2 \\
11 / 2 \\
9 / 2 \\
5 / 2\end{array}$ & $\begin{array}{l}\text { 1. } 25 \\
\text { 1. } 335 \\
\text { 1. } 405 \\
\text { 1. } 444 \\
\text { 1. } 107\end{array}$ & $\begin{array}{ll}\text { M } & 31 \\
\text { M } & 31 \\
\text { M } & 31 \\
\text { M } & 31 \\
\text { M } & 31\end{array}$ & $\begin{array}{l}4635 \\
4637 \\
4650 \\
4664 \\
4673\end{array}$ & $\begin{array}{l}46352.99 \\
46374.25 \\
46509.40 \\
46649.42 \\
46733.38\end{array}$ & $\begin{array}{l}7 / 2 \\
9 / 2 \\
5 / 2 \\
5 / 2 \\
3 / 2\end{array}$ & $\begin{array}{l}\text { 1. } 271 \\
\text { 1. } 341 \\
\text { 1. } 371 \\
\text { 1. } 334 \\
\text { 1. } 858\end{array}$ & $\begin{array}{ll}\text { M } & 31 \\
\text { M } & 31 \\
\text { M } & 31 \\
\text { M } & 31 \\
\text { K } & 47\end{array}$ \\
\hline $\begin{array}{l}3984 \\
3991 \\
3992 \\
4049\end{array}$ & $\begin{array}{l}39844.75 \\
39916.39 \\
39926.75 \\
40493.34\end{array}$ & $\begin{array}{r}7 / 2 \\
11 / 2 \\
3 / 2 \\
13 / 2\end{array}$ & $\begin{array}{l}\text { 1. } 223 \\
1.314 \\
0.745 \\
1.364\end{array}$ & $\begin{array}{ll}\text { M } & 31 \\
\text { M } & 31 \\
\text { K } & 47\end{array}$ & $\begin{array}{l}4700 \\
4710 \\
4717 \\
4718 \\
4720\end{array}$ & $\begin{array}{l}47004.34 \\
47101.61 \\
47172.1 \\
47172.9 \\
47205.73\end{array}$ & $\begin{array}{l}3 / 2 \\
5 / 2 \\
3 / 2 \\
1 / 2 \\
9 / 2\end{array}$ & $\begin{array}{l}\text { 1. } 285 \\
0.893 \\
\text { 2. } 215 \\
\text { 1. } 106\end{array}$ & $\begin{array}{ll}\text { M } & 31 \\
\text { K } & 47 \\
& \\
\text { M } & 31\end{array}$ \\
\hline $\begin{array}{l}4080 \\
4081 \\
4082 \\
4094 \\
4116\end{array}$ & $\begin{array}{l}40808.85 \\
40810.17 \\
40821.83 \\
40946.53 \\
41163.91\end{array}$ & $\begin{array}{l}3 / 2 \\
1 / 2 \\
5 / 2 \\
7 / 2 \\
7 / 2\end{array}$ & $\begin{array}{l}1.351 \\
-1.126 \\
1.534 \\
1.325\end{array}$ & $\begin{array}{ll}\text { M } & 31 \\
\text { M } & 31 \\
\text { M } & 31 \\
\text { M } & 31 \\
\text { M } & 31\end{array}$ & $\begin{array}{l}4735 \\
4750 \\
4765 \\
4766 \\
4770\end{array}$ & $\begin{array}{l}47358.36 \\
47506.79 \\
47664.74 \\
47669.01 \\
47703.78\end{array}$ & $\begin{array}{r}7 / 2 \\
13 / 2 \\
1 / 2 \\
7 / 2 \\
3 / 2\end{array}$ & $\begin{array}{l}\text { 1. } 151 \\
\text { 1. } 26 \\
\text { 1. } 50 \\
\text { 1. } 196 \\
\text { 1. } 443\end{array}$ & $\begin{array}{ll}\text { K } & 47 \\
\text { K } & 48 \\
& \\
\text { M } & 31 \\
\text { K } & 48\end{array}$ \\
\hline $\begin{array}{l}4131 \\
4145 \\
4155 \\
4184 \\
4199\end{array}$ & $\begin{array}{l}41313.02 \\
41453.18 \\
4155 \% .08 \\
41843.85 \\
41991.56\end{array}$ & $\begin{array}{l}5 / 2 \\
9 / 2 \\
3 / 2 \\
7 / 2 \\
1 / 2\end{array}$ & $\begin{array}{l}\text { 1. } 278 \\
\text { 1. } 372 \\
\text { 1. } 655 \\
\text { 1. } 190 \\
0.61\end{array}$ & $\begin{array}{ll}\text { M } & 31 \\
\text { M } & 31 \\
\text { M } & 31 \\
\text { M } & 31 \\
\text { K } & 48\end{array}$ & $\begin{array}{l}4777 \\
4785 \\
4789 \\
4793 \\
4797\end{array}$ & $\begin{array}{l}47779.91 \\
47859.93 \\
47899.22 \\
47932.55 \\
47970.82\end{array}$ & $\begin{array}{r}3 / 2 \\
11 / 2 \\
5 / 2 \\
7 / 2 \\
5 / 2\end{array}$ & $\begin{array}{l}\text { 1. } 362 \\
\text { 1. } 20 \\
\text { 1. } 217 \\
\text { 1. } 31 \\
\text { 1. } 169\end{array}$ & $\begin{array}{ll}\text { K } & 47 \\
\text { M } & 31 \\
\text { M } & 31 \\
\text { M } & 31 \\
\text { M } & 31\end{array}$ \\
\hline $\begin{array}{l}4214 \\
4225 \\
4304 \\
4334 \\
4340\end{array}$ & $\begin{array}{l}42140.06 \\
42254.19 \\
43044.02 \\
43341.85 \\
4340 \% .87\end{array}$ & $\begin{array}{l}9 / 2 \\
3 / 2 \\
5 / 2 \\
3 / 2 \\
7 / 2\end{array}$ & $\begin{array}{l}\text { 1. } 249 \\
\text { 1. } 578 \\
\text { 1. } 449 \\
0.975 \\
1.368\end{array}$ & $\begin{array}{ll}\mathrm{M} & 31 \\
\mathrm{~K} & 47 \\
\mathrm{~K} & 47 \\
\mathrm{M} & 31 \\
\mathrm{M} & 31\end{array}$ & $\begin{array}{l}4818 \\
4856 \\
4878 \\
4885 \\
4902\end{array}$ & $\begin{array}{l}48184.20 \\
48569.63 \\
48786.35 \\
4885 \% .60 \\
49022.77\end{array}$ & $\begin{array}{r}7 / 2 \\
11 / 2 \\
7 / 2 \\
7 / 2 \\
5 / 2\end{array}$ & $\begin{array}{l}\text { 1. } 252 \\
\text { 1. } 27 \\
\text { 1. } 53 \\
\text { 1. } 495\end{array}$ & $\begin{array}{ll}\text { M } & 31 \\
\text { M } & 31 \\
\text { K } & 47 \\
\text { K } & 47 \\
\text { M } & 31\end{array}$ \\
\hline $\begin{array}{l}4341 \\
4345 \\
4356 \\
4370 \\
4381\end{array}$ & $\begin{array}{l}43409.11 \\
43453.30 \\
43569.36 \\
43702.20 \\
43815.01\end{array}$ & $\begin{array}{l}3 / 2 \\
9 / 2 \\
5 / 2 \\
3 / 2 \\
7 / 2\end{array}$ & $\begin{array}{l}\text { 1. } 336 \\
1.504 \\
1.962 \\
1.348\end{array}$ & $\begin{array}{ll}\text { M } & 31 \\
\text { M } & 31 \\
\text { M } & 31 \\
\text { M } & 31 \\
\text { K } & 47\end{array}$ & $\begin{array}{l}4903 \\
4917 \\
4925 \\
4928 \\
4954\end{array}$ & $\begin{array}{l}49027.85 \\
49170.76 \\
49250.02 \\
49286.07 \\
49540.96\end{array}$ & $\begin{array}{r}7 / 2 \\
9 / 2 \\
3 / 2 \\
11 / 2 \\
5 / 2\end{array}$ & $\begin{array}{l}\text { 1. } 499 \\
\text { 1. } 135 \\
\text { 1. } 263 \\
\text { 1. } 075\end{array}$ & $\begin{array}{ll}\text { M } & 31 \\
\text { K } & 47 \\
\text { K } & 47 \\
\text { K } & 48\end{array}$ \\
\hline
\end{tabular}


TABLE 1. Energy levels of the Re atom-Continued

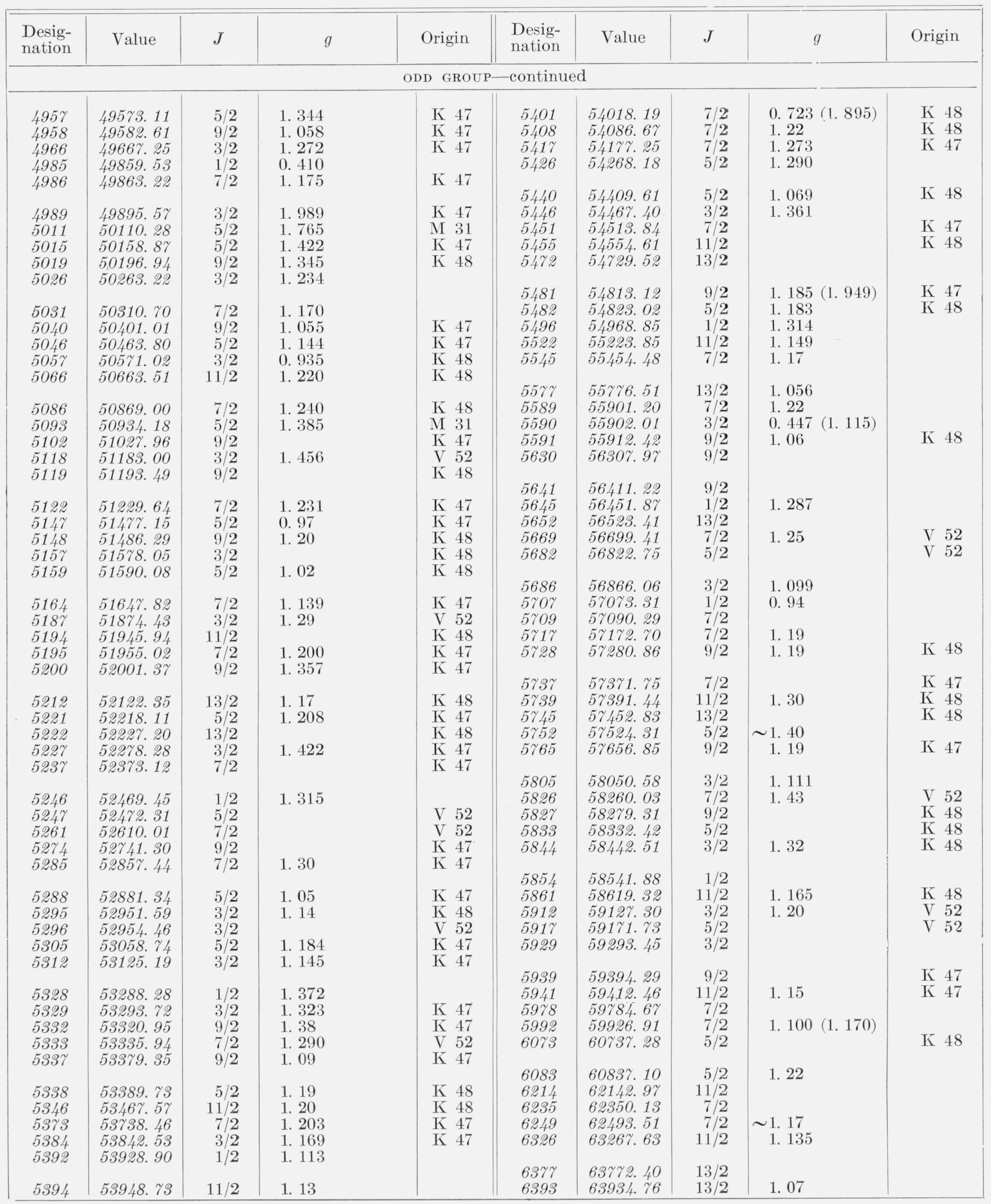

When no symbol is inserted in the column headed "origin" the corresponding level is a new one. The symbols ave the following meaning:

M 31, W. F. Meggers, BS J. Research 6, 1027 (1931) RP322.

M 33, W. F. Meggers, BS J. Research 6, 1027 (1931) RP322.

K 47, P. F. A. Klinkenberg, Physica 13, 581 (1947)

K 48, P. F. A. Klinkenberg, Physica 14, 269 (1948).

V 52, R. Velasco, Anales real soc. espan. fis. y quim. 48, 55 (1952). dated July $22,1953$. 
Table 2. Classified lines of Re I

\begin{tabular}{|c|c|}
\hline Int. & $\lambda_{\mathrm{vac}}$ \\
\hline (12) & $\underset{1716,45}{A}$ \\
\hline (12) & $\begin{array}{l}170.45 \\
1758.55\end{array}$ \\
\hline 3 & 1759.83 \\
\hline 0 & 1763.70 \\
\hline $1 \mathrm{w}$ & 1788.96 \\
\hline 1 & 1845.77 \\
\hline (12) & 1848.88 \\
\hline $0 \mathrm{~h}$ & 1851. 23 \\
\hline (3) & 1857.34 \\
\hline (5) & 1873.03 \\
\hline$(15 \mathrm{r})$ & 1874.93 \\
\hline 4 & 1876.36 \\
\hline (3) & 1884. 70 \\
\hline (10) & 1888. 43 \\
\hline (10) & 1891.04 \\
\hline$(50 \mathrm{R})$ & 1900.83 \\
\hline$(50 \mathrm{R})$ & 1905.74 \\
\hline$(50 \mathrm{R})$ & 1909. 36 \\
\hline $2 \mathrm{~h}$ & 1912. 81 \\
\hline 3 & 1915. 01 \\
\hline (15) & 1924.74 \\
\hline$(15 \mathrm{r})$ & 1927.72 \\
\hline 8 & 1941.33 \\
\hline$(5)$ & 1942.61 \\
\hline 12 & 1952.00 \\
\hline$(5 \mathrm{R})$ & 1953.77 \\
\hline$(15 \mathrm{R})$ & 1963.38 \\
\hline (5) & 1965.83 \\
\hline 0 & 1969.87 \\
\hline $2 \mathrm{~s}$ & 1976.80 \\
\hline 2 & 1977.48 \\
\hline$(5)$ & 1981. 62 \\
\hline$(20 \mathrm{R})$ & 1995. 61 \\
\hline $50 \mathrm{R}$ & $\begin{array}{c}\lambda_{\text {air }} \\
2003.532\end{array}$ \\
\hline (10) & 2004.84 \\
\hline (2) & 2012.74 \\
\hline $20 \mathrm{r}$ & 2016.56 \\
\hline $200 \mathrm{R}$ & 2017.866 \\
\hline & 2029. 70 \\
\hline $20 \mathrm{r}$ & 2033.78 \\
\hline $30 \mathrm{r}$ & 2038. 99 \\
\hline $50 \mathrm{r}$ & 2039. 204 \\
\hline (7) & 2046. 09 \\
\hline $400 \mathrm{R}$ & 2049.079 \\
\hline $20 \mathrm{r}$ & 2067.89 \\
\hline $20 \mathrm{r}$ & 2074.70 \\
\hline 5 & 2075.20 \\
\hline 7 & 2081.35 \\
\hline $40 \mathrm{r}$ & 2083.925 \\
\hline $200 \mathrm{R}$ & 2085. 594 \\
\hline $3 \mathrm{~h}$ & 2088.23 \\
\hline $50 \mathrm{r}$ & 2092. 24 \\
\hline 5 & 2094.50 \\
\hline & 2095.33 \\
\hline $100 \mathrm{R}$ & 2097. 122 \\
\hline 4 & 2097.603 \\
\hline 4 & 2098.41 \\
\hline 3 & 2102.67 \\
\hline 4 & 2106.13 \\
\hline $60 \mathrm{r}$ & 2107.452 \\
\hline $30 \mathrm{r}$ & 2113.87 \\
\hline 4 & 2128.773 \\
\hline 2 & 2131.01 \\
\hline 2 & 2133.122 \\
\hline 5 & 2133.41 \\
\hline $5 \mathrm{~h}$ & 2138.44 \\
\hline 6 & 2141.745 \\
\hline $20 \mathrm{r}$ & 2142.97 \\
\hline 3 & 2148.92 \\
\hline 5 & 2149.43 \\
\hline 1 & 2149. 59 \\
\hline 4 & 2151.40 \\
\hline $100 \mathrm{R}$ & 2156.673 \\
\hline 10 & 2163.10 \\
\hline $200 \mathrm{R}$ & 2167.938 \\
\hline $200 \mathrm{R}$ & 2176.206 \\
\hline & 2177.851 \\
\hline (5) & 2179.09 \\
\hline 8 & 2179.18 \\
\hline 20 & 2181.02 \\
\hline $100 \mathrm{R}$ & 2183.72 \\
\hline 3 & 2187.02 \\
\hline 6 & 2188.50 \\
\hline 10 & 2190.518 \\
\hline 40 & 2191.471 \\
\hline 3 & 2192.84 \\
\hline 5 & 2193.85 \\
\hline 8 & 2195.84 \\
\hline 10 & 2195.97 \\
\hline $200 \mathrm{R}$ & 2198. 914 \\
\hline 8 & 2201.075 \\
\hline 4 & \\
\hline 20 & 2207.680 \\
\hline 30 & 2209.800 \\
\hline 10 & 2212.809 \\
\hline 2 & 2213.50 \\
\hline
\end{tabular}


TABle 2. Classified lines of Re I-Continued

\begin{tabular}{|c|c|c|c|c|c|c|c|c|c|c|c|c|c|}
\hline Int. & $\lambda_{\mathrm{vac}}$ & $\sigma_{\mathrm{vac}}$ & Zeeman effect & Transition & $\mathrm{O}-\mathrm{C}$ & Notes & Int. & $\lambda_{\mathrm{vac}}$ & $\sigma_{\mathrm{vac}}$ & Zeeman effect & Transition & $\mathrm{O}-\mathrm{C}$ & Notes \\
\hline $30 \mathrm{r}$ & $\begin{array}{c}A \\
2353.95\end{array}$ & $\begin{array}{c}K \\
42468.8\end{array}$ & & $146_{5 / 2}-5709_{7 / 2}$ & .0 & $\pi-$ & $A_{5}$ & $\begin{array}{c}K \\
2447.656\end{array}$ & 40843.04 & & $150712-558997$ & -03 & \\
\hline $30 \mathrm{r}$ & 2354.08 & 42466.5 & & $150_{7 / 2}-5752_{5 / 2}$ & $\begin{array}{r}.0 \\
+.4\end{array}$ & - & 1 & 2448.26 & 40833.0 & & $\begin{array}{l}1007 / 2-55457 / 2 \\
1465 / 2-5547 / 2\end{array}$ & .00 & 5 \\
\hline 15 & 2354. 773 & 42453.96 & & $1737 / 2-59787 / 2$ & +.11 & - & $60 \mathrm{r}$ & 2449.710 & 40808. 79 & (000) 2.850 . & $0_{5 / 2}-4080_{3 / 2}$ & -.06 & 10,26 \\
\hline 10 & 2355.868 & 42434.21 & & $115_{5 / 2}-54017 / 2$ & -.02 & - & 30 & 2450.895 & 40789.07 & $(0 \mathrm{w}) ? \ldots$ & $115_{5 / 2}-5237_{7 / 2}$ & -.09 & -. \\
\hline $30 \mathrm{r}$ & 2356.496 & 42422.91 & & $117_{9 / 2}-5417_{7 / 2}$ & +.18 & -. & 8 & 2451.910 & 40772.19 & & $1669 / 2-5730_{11 / 2}$ & +.03 & -- \\
\hline 20 & 2362.64 & 42312.6 & & $163_{11 / 2}-5861_{11 / 2}$ & +.4 & $\ldots$ & 10 & 2453.493 & 40745.87 & $(.387)_{\ldots}$ & $164_{3 / 2}-5707_{1 / 2}$ & +.07 & -- \\
\hline 3 & 2364. 44 & 42280.4 & & $157_{5 / 2}-5805_{3 / 2}$ & +.2 & -. & 3 & 2454.082 & 40736. 10 & & $151_{1 / 2}-5590_{3 / 2}$ & -.02 & -- \\
\hline $20 \mathrm{r}$ & 2365.666 & 42258.47 & & $115_{5 / 2}-53843 / 2$ & -.10 & -. & 2 & 2458.577 & 40661.62 & & $1669 / 2-57289 / 2$ & +.04 & - \\
\hline $150 \mathrm{R}$ & 2365.90 & 42254.4 & $(.184, .556) \ldots$ & $0_{5 / 2}-42253 / 2$ & +.2 & -- & $\begin{array}{r}4 \\
20\end{array}$ & $\begin{array}{l}2459.81 \\
2460.24\end{array}$ & $\begin{array}{l}40641.3 \\
40634.2\end{array}$ & & $138_{3 / 2}-54463 / 2$ & .0 & -- \\
\hline & & & 2.496 & & & & $200 \mathrm{R}$ & 2461. 196 & 40618. 35 & (0h) $1.503 \mathrm{~h} \mathrm{D}$ & $117_{9 / 2}-5237_{7 / 2}$ & -.00 & -- \\
\hline $50 \mathrm{r}$ & 2367.683 & 42222.49 & (.000) $1.171 \ldots$ & $150_{7 / 2}-57288_{9 / 2}$ & -.18 & -- & 10 & 2462.539 & 40596.21 & $(.194, .574$ & $142_{7 / 2}-5481_{9 / 2}$ & -.05 & -- \\
\hline $\begin{array}{r}4 \\
15\end{array}$ & $\begin{array}{l}2368.12 \\
2368.88\end{array}$ & $\begin{array}{l}42214.8 \\
42201.2\end{array}$ & - & $\begin{array}{l}164_{3 / 2}-58541 / 2 \\
146_{5 / 2}-5682_{5 / 2}\end{array}$ & +.3 & -- & 15 & & & $.952)$. & $138, \quad 51 / 0$ & +06 & \\
\hline $50 \mathrm{r}$ & $\begin{array}{l}2308.88 \\
2369.27\end{array}$ & 42194.3 & $(.167, .500, .832)$ & $\begin{array}{r}140_{5 / 2}-558 \% 5 / 2 \\
142_{7 / 2}-5641_{9 / 2}\end{array}$ & $\begin{array}{l}-.1 \\
-.1\end{array}$ & $\overline{19}$ & 15 & 2463.307 & 40583.55 & $.445, .860$ & $138_{3 / 2}-54405 / 2$ & +.00 & -- \\
\hline $30 \mathrm{r}$ & 2371.500 & 42154.53 & $\begin{array}{r}.163, .493, .823 \\
(0 \mathrm{w}) 1.000 \mathrm{~B}\end{array}$ & $\left\{\begin{array}{c}117_{9 / 2}-539411 / 2 \\
115_{5 / 2}-537_{7 / 2}\end{array}\right.$ & $\begin{array}{l}+.1 \\
+.03\end{array}$ & 19 & 5 & 2463.84 & 40574.7 & & $217_{7 / 2}-62855_{7 / 2}$ & .0 & $\ldots$ \\
\hline 15 & 2373.747 & 42114.62 & (0) & $150_{7 / 2}-5 \gamma_{17} 7_{7 / 2}$ & +.11 & - & 30 & 2465.13 & 40553.5 & (000) 1.111 & $166_{9 / 2}-5 \gamma_{11} \gamma_{7 / 2}$ & +.1 & $\begin{array}{l}-- \\
--\end{array}$ \\
\hline $100 \mathrm{r}$ & 2375.073 & 42091.12 & & $142_{7 / 2}-5630_{9 / 2}$ & +.01 & .. & 5 & 2466.042 & 40538.54 & & $164_{3 / 2}-5686_{3 / 2}$ & -.01 & -- \\
\hline $15 \mathrm{r}$ & 2375.815 & 42077.97 & (0w) $1.412 \mathrm{hD}$ & $146_{5 / 2}-56697 / 2$ & +.02 & -.- & 4 & 2470.164 & 40470.90 & & $166_{9 / 2}-57097 / 2$ & -.20 & -- \\
\hline 10 & 2375.93 & 42075.9 & & $138_{3 / 2}-5590_{3 / 2}$ & .0 & -. & 3 & 2470.275 & 40469.08 & & $194_{5 / 2}-5992_{7 / 2}$ & +.06 & -- \\
\hline 10 & 2376.629 & 42063.57 & & $1737 / 2-59399_{9 / 2}$ & +.10 & -. & 2 & 2471.93 & 40441.9 & & $138_{3 / 2}-5426_{5 / 2}$ & -.2 & -- \\
\hline 10 & 2377.104 & 42055.15 & & $172_{1 / 2}-5929_{3 / 2}$ & .00 & .. & 15 & 2474.725 & 40396. 32 & (000) 1.154. & $150_{7 / 2}-55457 / 2$ & +.03 & -- \\
\hline 3 & 2378.40 & 42032.2 & & $150_{7 / 2}-5 \% 09_{7 / 2}$ & +.1 & -. & 5 & 2476.275 & 40371.04 & & $115_{5 / 2}-51957 / 2$ & -.02 & - \\
\hline 5 & 2379.950 & 42004.87 & & $164_{3 / 2}-5833_{5 / 2}$ & -.04 & -. & $2 \mathrm{~h}$ & 2478.58 & 40333.5 & & $221_{9 / 2}-6249_{7 / 2}$ & .0 & -- \\
\hline 20 & 2380.225 & 42000.02 & (.006) 1.124 & $1669 / 2-5861_{11 / 2}$ & -.02 & -. & 20 & 2479.02 & 40326.3 & & $1945 / 2-59787 / 2$ & -.5 & - \\
\hline 40 & 2381. 136 & 41983.96 & $(.159, .488, .817)$ & $117_{9 / 2}-5373_{7 / 2}$ & +.02 & -. & 20 & & 40526.3 & & $1737 / 2-5765_{9 / 2}$ & +.3 & -- \\
\hline 6 & 2381.81 & 41972.0 & - & $163_{11 / 2}-582 \gamma_{9 / 2}$ & -.2 & -- & 20 & 2480.820 & 40297.08 & $(.471, .792$, & $1427 / 2-54517 / 2$ & +.1 & -- \\
\hline $\begin{array}{l}15 \\
10\end{array}$ & $\begin{array}{l}2385.48 \\
2386.536\end{array}$ & $\begin{array}{l}41907.5 \\
41888.95\end{array}$ & $-\cdots$ & $\begin{array}{l}151_{1 / 2}-5707_{1 / 2} \\
172_{1 / 2}-5912_{3 / 2}\end{array}$ & $\begin{array}{l}+.1 \\
-.05\end{array}$ & -- & 4 & 2481.226 & 40290.49 & $1.114)$ & $115_{5 / 2}-5187_{3 / 2}$ & +02 & $\ldots$ \\
\hline $20 \mathrm{r}$ & 2389.110 & 41843.84 & $(.376,1.118)$ & $0_{5 / 2}-41847 / 2$ & +.61 & - & 5 & 2483.38 & 40255.5 & & $204_{3 / 2}-6073_{5 / 2}$ & -.1 & - \\
\hline 5 & 2389.270 & 41841.03 & & $1737 / 2-5917_{5 / 2}$ & +.08 & -- & $200 \mathrm{R}$ & 2483.920 & 40246. 79 & $(.875) 1.452 \mathrm{~A}$ & $1179 / 2-5200_{9 / 2}$ & -.06 & - \\
\hline $30 \mathrm{r}$ & 2391.278 & 41805.89 & & $115_{5 / 2}-5338_{5 / 2}$ & +.12 & -. & 20 & 2485.805 & 40216. 28 & & $163_{11 / 2}-5652_{13 / 2}$ & +.02 & -- \\
\hline $50 \mathrm{r}$ & 2393.645 & 41764.56 & & $1507 / 2-5682_{5 / 2}$ & .00 & -- & 30 & 2486.779 & 40200.53 & (.171, .490, & $117_{9 / 2}-5195_{7 / 2}$ & +.03 & -. \\
\hline 4 & 2394. 252 & 41753.98 & & $157_{5 / 2}-5752_{5 / 2}$ & +.09 & -- & & & & $.809)$ & & & \\
\hline $30 \mathrm{r}$ & 2394. 37 & 41751.9 & . & $115_{5 / 2}-5333_{7 / 2}$ & -.1 & -- & 5 & 2487. 21 & 40193. 6 & & $173_{7 / 2}-5752_{5 / 2}$ & +.1 & -- \\
\hline 7 & 2396.03 & 41723.0 & - & $164_{3 / 2}-5805_{3 / 2}$ & -.1 & & $200 \mathrm{R}$ & 2487.331 & 40191.60 & & $-519411 / 2$ & +.18 & -- \\
\hline (10) & 2396.60 & 41713. 1 & & $117_{9 / 2}-5346_{11 / 2}$ & .0 & 7 & 9 & 2491.07 & 40131.3 & & $157_{5 / 2}-5590_{3 / 2}$ & -.3 & -- \\
\hline $40 \mathrm{r}$ & 2396. 791 & 41709. 74 & (.000) $1.245_{\ldots}$ & $115_{5 / 2}-5329_{3 / 2}$ & -.02 & $-\frac{1}{8}$ & & & & & $157_{5 / 2}-5589_{7 / 2}$ & +.5 & -- \\
\hline 50 & 2397.31 & 41700.7 & (.626) .465 & $151_{1 / 2}-5686_{3 / 2}$ & +.5 & 8 & 2 & 2491. 497 & 40124. 40 & & $1643 / 2-56451 / 2$ & +.04 & -- \\
\hline 4 & 2397.605 & 41695.59 & & $142_{7 / 2}-5591_{9 / 2}$ & +.03 & -. & 8 & .751 & & & $163_{11 / 2}-5641_{9 / 2}$ & +.15 & -- \\
\hline 10 & 2398.25 & 41684.4 & & $142_{\tau / 2}-55899_{7 / 2}$ & +.1 & -- & 3 & .838 & 40102.82 & ـ ـ 1.738 . & $138_{3 / 2}-5392_{1 / 2}$ & +.04 & -- \\
\hline 6 & 2399.648 & 4166 & & $166_{9 / 2}-582 \gamma_{9 / 2}^{\prime}$ & +.07 & -. & 3 & 2494.250 & 40080.12 & & $166_{9 / 2}-5669_{7 / 2}$ & -.01 & -. \\
\hline 20 & 2400.72 & 41641.5 & & $150_{7 / 2}-5669_{7 / 2}$ & +.3 & -- & 8 & 2495.263 & 40063.84 & & $115_{5 / 2}-51647 / 2$ & -.02 & \\
\hline 30 & 2401.680 & 41624.85 & $\begin{array}{l}(.705,1.178 \\
1.650,2.125)\end{array}$ & $117_{9 / 2}-5337_{9 / 2}$ & +.02 & -- & 30 & 2496.039 & 40051.39 & $\begin{array}{c}.142, .419 \\
.697) .835 \dagger\end{array}$ & $1427 / 2-5426_{5 / 2}$ & +.07 & 11 \\
\hline & & & $.388, .847$ & & & & 10 & 2496. 692 & 40040.92 & & $173_{7 / 2}-5737_{7 / 2}$ & -.01 & - \\
\hline 10 & 2403.036 & 41601.36 & & $157_{5 / 2}-5737_{7 / 2}$ & +.03 & 5 & 20 & & & $\begin{array}{l}\text { (.479) } 1.010 \\
1.345,1.678^{+} .\end{array}$ & & +.10 & -- \\
\hline $300 \mathrm{R}$ & 2405.056 & 41566.42 & (.730) $1.547 \mathrm{WA}$ & $117_{9 / 2}-5332_{9 / 2}$ & -.01 & -- & 20 & 2498.861 & $400 \mathrm{c} 6.17$ & $(.394, .661)$ & $115_{5 / 2}-5159_{5 / 2}$ & +.05 & 12 \\
\hline $200 \mathrm{r}$ & 2405.602 & 41556.99 & $(.152, .444)$ & $0_{5 / 2}-4155_{3 / 2}$ & -.69 & -- & & & 40001,0 & 1.386 & (2) & +2 & - \\
\hline & & & 2.386 & & & & $\begin{array}{l}1 \\
2\end{array}$ & 608 & & & 115 & $\begin{array}{r}.213 \\
+.13\end{array}$ & - \\
\hline $30 \mathrm{r}$ & 2410.37 & 41474.8 & (.210) $1.235 \mathrm{~h}$ & $115_{5 / 2}-5305_{5 / 2}$ & .0 & -- & 10 & & & & $411 / 2$ & +.02 & -. \\
\hline $30 \mathrm{r}$ & 2414.587 & 41402.36 & $(0 \mathrm{w}) .965 \mathrm{hC}_{\ldots}$ & $-5 \gamma_{11} \gamma_{7 / 2}$ & +.08 & -- & 10 & $25 C 0.568$ & 39978.85 & $(0 w) D \ldots$ & $239_{11 / 2}-6393_{13 / 2}$ & +.09 & -. \\
\hline $4 \mathrm{~h}$ & 2415.93 & 41379. 4 & & $194_{5 / 2}-6083_{5 / 2}$ & +.2 & -. & $50 \mathrm{r}$ & 2501.721 & 39960.43 & $(.453, .759$ & $142_{7 / 2}-5417_{7 / 2}$ & +.04 & -- \\
\hline 10 & 2416.441 & 41370.60 & -1 & $115_{5 / 2}-52066_{3 / 2}$ & +.10 & -- & & & & 1.062).529, & & & \\
\hline 15 & 2417.661 & 41349. 72 & (0w) $1.438 \mathrm{D}$ & $163_{11 / 2}-57659 / 2$ & +.02 & -- & & & & $.819,1.113$ & & & \\
\hline $60 \mathrm{r}$ & 2419.404 & 41319. 94 & & $157_{5 / 2}-57099_{7 / 2}$ & +.07 & -- & & & & $1.417,1.707$ & & & \\
\hline $100 \mathrm{r}$ & 2419.807 & 41313.06 & $\begin{array}{c}(.335,1.004, \\
1.673) .287 \\
956,1616\end{array}$ & $05 / 2-4131_{5 / 2}$ & +.04 & -- & 30 & 2505.945 & 39893.08 & $\begin{array}{l}1.992 . \\
(.485, .805) \\
1.146 .\end{array}$ & $115_{5 / 2}-51 / 7_{5 / 2}$ & -.11 & -- \\
\hline 5 & 2420.35 & 41303. 7 & $2.279,2.948$. & $172_{1 / 2}-5854 / 1 / 2$ & +.1 & $\ldots$ & 30 & 2507.403 & 39869.89 & $\begin{array}{l}(.566, .907 \\
1.251)\end{array}$ & $142_{7 / 2}-5408_{7 / 2}$ & +.08 & 12 \\
\hline 5 & 2420.72 & 41297. 4 & & $115_{5 / 2}-5288_{5 / 2}$ & $\begin{array}{r}\text { T. } \\
.0\end{array}$ & - & $200 \mathrm{R}$ & 2508.991 & 39844.65 & $(.366,1.094$ & $0_{5 / 2}-39847 / 2$ & +.10 & 12 \\
\hline 30 & 2421.38 & 41286.1 & & $151_{1 / 2}-56455_{1 / 2}$ & +.1 & -- & & & & $1.821) /$ & & & \\
\hline $40 \mathrm{r}$ & 2421.730 & 41280.26 & $\left\{\begin{array}{l}(0 \mathrm{w}) 1.198 \ldots \\
.\end{array}\right.$ & $\begin{array}{l}146_{5 / 2}-5590_{3 / 2} \\
146_{5 / 2}-55899_{7 / 2}\end{array}$ & $\left.\begin{array}{l}-.29 \\
+.52\end{array}\right\}$ & 6 & $\begin{array}{r}5 \\
(5)\end{array}$ & $\begin{array}{l}2509.562 \\
2510.771\end{array}$ & $\begin{array}{l}39835.59 \\
39816.41\end{array}$ & & $\begin{array}{l}194_{5 / 2}-5929_{3 / 2} \\
239_{11 / 2}-6377_{13 / 2}\end{array}$ & $\begin{array}{l}+.03 \\
+.01\end{array}$ & 7 \\
\hline 2 & 2422.11 & 41273.7 & & $115_{5 / 2}-5285_{7 / 2}$ & $\begin{array}{l}.02 \\
+.2\end{array}$ & -. & 5 & 2511. 616 & 39803.02 & & $151_{1 / 2}-5496_{1 / 2}$ & +.06 & -. \\
\hline 30 & & 41250.0 & & $15 \mathrm{C}_{7 / 2}-5630_{9 / 2}$ & +.2 & .. & 2 & & & & $142_{7 / 2}-5401_{7 / 2}$ & +.64 & -- \\
\hline 10 & 2426.195 & 41204. 28 & & $172_{1 / 2}-58$ & +.07 & & 4 & 2512.317 & 1. 92 & & $166_{9 / 2}-564_{1} 1_{9 / 2}$ & -.02 & -- \\
\hline 30 & 2426.635 & 41196.81 & $(000) .994$ & $164_{3 / 2}-5752_{5 / 2}$ & +.01 & 11 & 15 & 2512.550 & 39788. 23 & (.210) $1.051 \mathrm{~A}$ & $146_{5 / 2}-5440_{5 / 2}$ & +.08 & -- \\
\hline $300 \mathrm{R}$ & 2428.576 & 41163.90 & $\begin{array}{l}(.319, .972 \\
1.625), .319\end{array}$ & $0_{5 / 2}-4116_{7 / 2}$ & -.01 & -- & $\begin{array}{r}2 \mathrm{~h} \\
5\end{array}$ & $\begin{array}{l}2514.35 \\
2514.651\end{array}$ & $\begin{array}{l}39759.7 \\
39754.98\end{array}$ & & $\begin{array}{l}1737 / 2-5709_{7 / 2} \\
150_{7 / 2}-5481_{9 / 2}\end{array}$ & $\begin{array}{l}+.2 \\
+.05\end{array}$ & -. \\
\hline & & & $\begin{array}{l}.965,1.611, \\
2.255 \text {. }\end{array}$ & & & & $150 \mathrm{r}$ & 2516.120 & 39731.77 & $\begin{array}{l}(.506, .857 \\
1.209,1.558)\end{array}$ & $117_{9 / 2}-51489 / 2$ & .0 & -. \\
\hline 10 & 2429.648 & 41145. 74 & & $163_{11 / 2}-5745_{13 / 2}$ & +.06 & -- & & & & $000, .341, .682$ & & & \\
\hline 80 & 2432.18 & & $\begin{array}{c}(.125, .412 \\
.696)\end{array}$ & $117_{9 / 2}-52857 / 2$ & & & & & & $\begin{array}{l}1.023,1.368 \\
1.708,2.043\end{array}$ & & & \\
\hline 5 & 2432.617 & 41095.52 & & $157_{5 / 2}-5 t$ & -.08 & -- & & & & $2.384,2.729$ & & & \\
\hline 20 & 2433.28 & 41084.3 & (.172) $1.256 \mathrm{~h}$ & $163_{11 / 2}-57399_{11 / 2}$ & .0 & -- & 5 & 2518.87 & 39688.4 & & $166_{9 / 2}-56$ & -.3 & -- \\
\hline 10 & 2436.046 & 41037.67 & . 1.182 & $166_{9 / 2}-5765_{9 / 2}$ & +.10 & & 6 & 2519.139 & & & $157_{5 / 2}-55457 / 2$ & +.10 & -- \\
\hline (10) & 2436.74 & 41026.0 & (000) 1.095 & $115_{5 / 2}-52617 / 2$ & -.1 & 7 & $50 \mathrm{r}$ & 2520.009 & 39670.46 & $(.417,1.266$ & $0_{5 / 2}-3967_{5 / 2}$ & -.06 & -- \\
\hline 30 & 2438.462 & 40997.03 & $\begin{array}{l}(.156, .467) \\
.715,1.026 \text {, } \\
1.338 .\end{array}$ & $138_{3 / 2}-5482_{5 / 2}$ & +.13 & -- & & & & $\begin{array}{l}2.112)-.146 \\
.684, \mathbf{1 . 5 2 6} \\
2.369,3.211\end{array}$ & & & \\
\hline 30 & 2439.06 & 40987.0 & & $117_{9 / 2}-52 \gamma_{49 / 2}$ & +.2 & -. & 10 & & 39647.0 & & $146_{5 / 2}-5426_{5 / 2}$ & +.3 & -- \\
\hline 3 & 2439.86 & & & $163_{11 / 2}-5728_{9 / 2}$ & -.2 & & 5 & 1. 59 & & & $115_{5 / 2}-51227 / 2$ & -.1 & -- \\
\hline & 2441.35 & & & $173_{7 / 2}-5827_{9 / 2}$ & .0 & 5 & 5 & 2522.719 & 39627.84 & & $172_{1 / 2}-5686_{3 / 2}$ & +.08 & -- \\
\hline $100 \mathrm{R}$ & 2441.47 & 40946.5 & $(.211, .628$ & $0_{5 / 2}-40947 / 2$ & .0 & -- & 5 & 2524.155 & 39605.30 & & $163_{11 / 2}-5591_{9 / 2}$ & +.03 & -- \\
\hline & & & 1.046) .490, & & & & $\begin{array}{r}6 \\
10\end{array}$ & 2524.546 & 39599.17 & & $115_{5 / 2}-511$ & +.13 & -- \\
\hline & & & $\begin{array}{l}.906,1.319, \\
1.730,2.143\end{array}$ & & & & $\begin{array}{r}10 \\
5\end{array}$ & $\begin{array}{l}2526.809 \\
2527.311\end{array}$ & $\begin{array}{l}39563.70 \\
39555.85\end{array}$ & & $\begin{array}{l}138_{3 / 2}-53388_{5 / 2} \\
146_{5 / 2}-5417_{7 / 2}\end{array}$ & $\begin{array}{l}+.09 \\
+.06\end{array}$ & -- \\
\hline & & & $\begin{array}{l}2.556 \text {. } \\
\text {. }\end{array}$ & & & & (1) & 2528.62 & 39535.4 & & $197_{1 / 2}-59299_{3 / 2}$ & -.1 & $\overline{5}$ \\
\hline $40 \mathrm{r}$ & 2442.510 & 40929,09 & & $173_{7 / 2}-58267 / 2$ & $-.12\}$ & 9 & 15 & 2529.50 & 39521.6 & & $1427 / 2-5373_{7 / 2}$ & .0 & -- \\
\hline 701 & & & (000) $1.129 \ldots$ & $157_{5 / 2}-56699_{7 / 2}$ & $+.10\}$ & & 5 & 2531. 404 & 39491.89 & & $1737 / 2-56825 / 2$ & -.04 & -- \\
\hline & & $\begin{array}{l}40888.35 \\
40854.36\end{array}$ & & $\begin{array}{l}115_{5 / 2}-5247_{5 / 2} \\
1507-55919 / 2\end{array}$ & +13 & -- & $\begin{array}{l}5 \\
5\end{array}$ & $\begin{array}{l}2532.48 \\
2532.848\end{array}$ & $\begin{array}{l}39475.1 \\
39469.38\end{array}$ & & $\begin{array}{l}117_{9 / 2}-5122_{7 / 2} \\
163_{11 / 2}-557 \gamma_{13 / 2}\end{array}$ & +.02 & \\
\hline
\end{tabular}


TABLE 2. Classified lines of Re I-Continued

\begin{tabular}{|c|c|c|c|c|c|c|c|c|c|c|c|c|c|}
\hline Int. & $\lambda_{\mathrm{vac}}$ & $\sigma_{\mathrm{Vac}}$ & Zeeman effect & Transition & $\mathrm{O}-\mathrm{C}$ & Notes & Int. & $\lambda_{\mathrm{vac}}$ & $\sigma_{\mathrm{vac}}$ & Zeeman effect & Transition & $\mathrm{O}-\mathrm{C}$ & Notes \\
\hline & & & & & & & & $A$ & $K$ & & & & \\
\hline $\begin{array}{l}4 \\
4\end{array}$ & $\begin{array}{l}2532.963 \\
2533.119\end{array}$ & $\begin{array}{l}39467.59 \\
39465.16\end{array}$ & & $138_{3 / 2}-5329_{3 / 2}$ & $\begin{array}{l}-.01 \\
-.05\end{array}$ & -- & $\begin{array}{r}5 \\
10\end{array}$ & $\begin{array}{l}2611.702 \\
2612.730\end{array}$ & $\begin{array}{l}38277.77 \\
38262.71\end{array}$ & & $\begin{array}{l}1507 / 2-53337 / 2 \\
1507 / 2-53320 / 9\end{array}$ & +.02 & -- \\
\hline $\begin{array}{r}4 \\
15 r\end{array}$ & $\begin{array}{l}2030.119 \\
2533.310\end{array}$ & $\begin{array}{l}59400.10 \\
39462.19\end{array}$ & & $\begin{array}{l}1465 / 2-5408_{7 / 2} \\
138_{3 / 2}-5328_{1 / 2}\end{array}$ & $\begin{array}{l}-.05 \\
+.03\end{array}$ & -- & $\begin{array}{r}10 \\
5\end{array}$ & 2612.929 & $\begin{array}{l}38262.71 \\
38259.80\end{array}$ & & $\begin{array}{l}150_{7 / 2}-53822_{9 / 2} \\
146_{5 / 2}-52885 / 2\end{array}$ & $\begin{array}{l}-.05 \\
-.08\end{array}$ & -- \\
\hline 1 & 2533.74 & 39455.5 & & $150_{7 / 2}-54517 / 2$ & -.2 & & 10 & 2613. 235 & 38255.32 & & $1427 / 2-5247_{5 / 2}$ & -.13 & -- \\
\hline 30 & 2534.805 & 39438. 91 & $(260, .775$ & $117_{9 / 2}-5119_{9 / 2}$ & -.06 & 12 & $\begin{array}{r}15 \\
50 \mathrm{R}\end{array}$ & $\begin{array}{l}2613.744 \\
2614.558\end{array}$ & $\begin{array}{c}38247.87 \\
38235.96\end{array}$ & $\begin{array}{l}(.293) \mathrm{A} \\
(0 \mathrm{w}) 1.662 \mathrm{~B}\end{array}$ & $\begin{array}{l}157_{5 / 2}-54017 / 2 \\
1465 / 2-52857 / 2\end{array}$ & $\begin{array}{l}+.10 \\
-\quad 02\end{array}$ & - \\
\hline 4 & 2537.517 & 39396. 77 & & $146_{5 / 2}-54017 / 2$ & +.04 & -- & 10 & 2617. 442 & 38193.84 & & $1669 / 2-5481_{9 / 2}$ & .00 & -- \\
\hline 20 & 2539.326 & 39368. 69 & & $173_{7 / 2}-5669_{7 / 2}$ & +.10 & -- & $40 \mathrm{R}$ & 2620.026 & 38156.17 & (.341) $1.485 \mathrm{~A}$ & $1427 / 2-52377 / 2$ & -.09 & \\
\hline 3 & 2540.45 & 39351.3 & & $1507 / 2-5440_{5 / 2}$ & -.1 & -- & $30 \mathrm{R}$ & 2620.344 & 38151.54 & (.118) $1.816 \ldots$ & $217_{7 / 2}-5992_{7 / 2}$ & +.03 & 11 \\
\hline $40 \mathrm{r}$ & 2540.513 & 39350.31 & (.252) $1.342 \mathrm{~A}_{--}$ & $115_{5 / 2}-5093_{5 / 2}$ & +.09 & -- & 10 & 2621. 144 & 38139. 90 & & $164_{3 / 2}-54466_{3 / 2}$ & +.01 & -- \\
\hline $20 \mathrm{~h}$ & 2541.27 & 39338.6 & 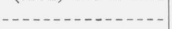 & $231_{5 / 2}-62497 / 2$ & -1 & -- & 15 & 2621.975 & 38127.81 & $(.510) \ldots$ & $151_{1 / 2}-5329_{3 / 2}$ & -.02 & -- \\
\hline 2 & 2543.022 & 39311. 48 & $(495) 866$ & $239_{11 / 2}-63266_{11 /:}$ & -.15 & $-\overline{5}$ & 20 & 2623. 285 & 38108.76 & $(.185, .554$ & $117_{9 / 2}-49867 / 2$ & +.06 & -- \\
\hline $\begin{array}{r}10 \\
8\end{array}$ & $\begin{array}{l}2543.671 \\
2543.835\end{array}$ & $\begin{array}{l}39301.45 \\
39298.92\end{array}$ & (.495) $.866 \ldots$ & $\begin{array}{l}151_{1 / 2}-544 b_{3 / 2} \\
138_{3 / 2}-5312_{3 / 2}\end{array}$ & $\begin{array}{l}-.06 \\
-.15\end{array}$ & 5 & 8 & 2625.041 & 38083,28 & .919) & $115_{5 / 2}-4966_{3 / 2}$ & +.09 & \\
\hline 10 & 2544.215 & 39293.05 & $(.736) \mathrm{A}$ & $1669 / 2-55919 / 2$ & -.09 & - & 3 & 2625.128 & 38082.02 & & $164_{3 / 2}-5440_{5 / 2}$ & -.08 & - \\
\hline $100 \mathrm{r}$ & 2544.739 & 39284. 96 & (0w) $1.169 \mathrm{D}_{\text {- - }}$ & $115_{5 / 2}-50867 / 2$ & -.08 & -- & 7 & 2625.806 & 38072.19 & & $1575 / 2-53843 / 2$ & +.08 & -- \\
\hline 8 & 2544.952 & 39281.67 & (0w) 1.047 & $166_{9 / 2}-5589_{7 / 2}$ & -.25 & -- & 1 & 2626.200 & 38066.48 & & $1945 / 2-57525 / 2$ & +.06 & -- \\
\hline $30 \mathrm{r}$ & 2545.485 & 39273.45 & $\begin{array}{l}(.993,1.447, \\
1.898)\end{array}$ & $1177_{9 / 2}-5102 g_{9 / 2}$ & +.01 & -- & $\begin{array}{l}5 \\
1\end{array}$ & $\begin{array}{l}2626.636 \\
2627.450\end{array}$ & $\begin{array}{l}38060.16 \\
38048,36\end{array}$ & & $\begin{array}{l}204_{3 / 2}-58541 / 2 \\
138_{3 / 2}-5187_{3 / 2}\end{array}$ & $\begin{array}{r}+.01 \\
+.05\end{array}$ & - \\
\hline 10 & 2548.136 & 39232.59 & $(.151, .457)$ & $138_{3 / 2}-5305_{5 / 2}$ & -.03 & -- & 8 & 2630.150 & 38009.31 & & $217_{7 / 2}-5978_{7 / 2}$ & +.04 & - \\
\hline & & & $.762,1.068$ & & & & 7 & 2630.754 & 38000.59 & & $1507 / 2-53055 / 2$ & +.04 & - \\
\hline $\begin{array}{r}20 \\
6\end{array}$ & & $\begin{array}{l}39221.06 \\
39213.57\end{array}$ & (000) $1.143 \ldots$ & $146_{5 / 2}-538 / 3 / 2$ & -.01 & -- & 20 & 2631. 569 & 37988.81 & & $115_{5 / 2}-4957_{5 / 2}$ & -.36 & -- \\
\hline $40 \mathrm{r}$ & $\begin{array}{l}2549.372 \\
2552.021\end{array}$ & $\begin{array}{l}39213.57 \\
39172.87\end{array}$ & $(.189, .575$ & $\begin{array}{l}172_{1 / 2}-5645_{1 / 2} \\
142_{7 / 2}-5338_{5 / 2}\end{array}$ & $\begin{array}{l}.00 \\
.00\end{array}$ & -- & $\begin{array}{l}10 \\
20\end{array}$ & $\begin{array}{l}2633.006 \\
2633.614\end{array}$ & $\begin{array}{l}37968.08 \\
37959.32\end{array}$ & $(.611) .555 \ldots$ & $\begin{array}{l}157_{5 / 2}-53737 / 2 \\
151_{1 / 2}-5312_{3 / 2}\end{array}$ & $\begin{array}{l}+.04 \\
+.02\end{array}$ & -- \\
\hline & & 5918.86 & $.961) 1.018$ & & & -- & $(2 \mathrm{~h})^{\circ}$ & 2633.775 & 37957.00 & & $115_{5 / 2}-49545 / 2$ & .00 & 7 \\
\hline & & & $\begin{array}{l}1.396,1.74, \\
2.152,2.526 .\end{array}$ & & & & $(1)^{2}$ & $\begin{array}{l}2634.908 \\
2635.29\end{array}$ & $\begin{array}{l}37940.68 \\
37935.2\end{array}$ & & $\begin{array}{l}1643 / 2-54265 / 2 \\
1669 / 2-5455_{11 / 2}\end{array}$ & $\begin{array}{l}+.01 \\
-.1\end{array}$ & $1 \overline{3}$ \\
\hline 6 & 2554. 929 & 39128. 29 & & $138_{3 / 2}-5296_{3 / 2}$ & -.05 & -- & 2 & 2636.019 & 37924.68 & & $244_{5 / 2}-62355_{7 / 2}$ & -.05 & -. \\
\hline 2 & 2555.116 & 39125.42 & & $138_{3 / 2}-5295_{3 / 2}$ & -.05 & -- & $100 \mathrm{R}$ & 2636.637 & 37915.79 & $(.227, .691) \ldots$ & $0_{5} / 2-3791_{3 / 2}$ & -.08 & -- \\
\hline 4 & 2555.531 & 39119.06 & & $\begin{array}{l}1427 / 2-53337 / 2 \\
150_{7 / 2}-5417_{7 / 2}\end{array}$ & $\begin{array}{r}-.02 \\
.00\end{array}$ & -- & & & & $.726,1.164$ & $\int 146_{5 / 2}-5247_{7 / 2}$ & -.17 & \\
\hline 5 & 2555.666 & 39117.00 & & $146_{5 / 2}-537 S_{7 / 2}$ & .00 & - & 10 & 2641.173 & 37850.68 & & $\left\{\begin{array}{l}204_{3 / 2}-5833_{5 / 2}\end{array}\right.$ & -01 & - \\
\hline $80 \mathrm{R}$ & 2556.512 & 39104.06 & $\begin{array}{l}(.101, .302, \\
.502, .803) \\
.697, .893, \\
1.090,1.286 \\
1.483,1.679 .\end{array}$ & $142_{7 / 2}-5332_{9 / 2}$ & -.03 & - & $60 \mathrm{R}$ & 2642. 752 & 37828.07 & $\begin{array}{l}(.754,1.217, \\
1.679,2.144) \\
.349, .821, \\
1.293,1.765, \\
2.237\end{array}$ & $117_{9 / 2}-4958_{9 / 2}$ & -.02 & - \\
\hline $\begin{array}{r}10 \\
20 \mathrm{R}\end{array}$ & $\begin{array}{l}2558.062 \\
2559.076\end{array}$ & $\begin{array}{l}39080.37 \\
39064.88\end{array}$ & $(291,916$ & $\begin{array}{r}173_{7 / 2}-56411_{9 / 2} \\
05 / 2-3906_{5 / 2}\end{array}$ & $\begin{array}{l}-.03 \\
-.04\end{array}$ & -- & $\begin{array}{l}3 \\
5\end{array}$ & $\begin{array}{l}2643.092 \\
2644.768\end{array}$ & $\begin{array}{l}37823.21 \\
37799.23\end{array}$ & & $\begin{array}{l}150_{7 / 2}-5288_{5 / 2} \\
150_{7 / 2}-52857 / 2\end{array}$ & $\begin{array}{l}+.06 \\
-.02\end{array}$ & -- \\
\hline & & & 1.540) 406 & & & & 10 & 2645.804 & 37784.44 & & $1427 / 2-520 G_{9 / 2}$ & -.07 & -- \\
\hline & & & $\begin{array}{l}\text { 1.031, 1.656, } \\
2.280,2.903 \text {. }\end{array}$ & & & & $30 \mathrm{R}$ & 2647. 128 & 37765.54 & $\begin{array}{l}(.302, .905, \\
1.508)\end{array}$ & $0_{5} / 2-3776_{7 / 2}$ & -.11 & -. \\
\hline 6 & & $\begin{array}{l}39055.25 \\
3905.58\end{array}$ & - & $138_{3 / 2}-5288_{5 / 2}$ & +.03 & -- & 2 & 2647. 240 & 37763.95 & & $138_{3 / 2}-5159_{5 / 2}$ & -.01 & -- \\
\hline 6 & $\begin{array}{l}2559.882 \\
2561.462\end{array}$ & $\begin{array}{l}39052.58 \\
39028.50\end{array}$ & (.159) $1.162 \mathrm{~A}$ & & -.02 & -. & 7 & 2648. 100 & 37751.67 & & $146_{5 / 2}-5237_{7 / 2}$ & +.01 & -- \\
\hline $50 \stackrel{8}{R}$ & & $\begin{array}{l}39028.50 \\
38987.03\end{array}$ & $\begin{array}{l}(.159) 1.162 \mathrm{~A}_{-.} \\
(.167, .505)\end{array}$ & $1507 / 2-54087 / 2$ & +.02 & -- & $50 \mathrm{R}$ & 2649.050 & 37738.14 & $(.564, .934$, & $1427 / 2-51957 / 2$ & -.02 & -- \\
\hline & & & $\begin{array}{l}.781,1.113 \\
1.446,1.778\end{array}$ & $115_{5 / 2}-5057_{3 / 2}$ & -.03 & -- & & & & $\begin{array}{l}1.009,1.379 \\
1.749,2.118\end{array}$ & & & \\
\hline 10 & 2564. 347 & 38984.59 & - & $194_{5 / 2}-58443 / 2$ & -.03 & -- & 10 & 2649.584 & 37730.53 & & $172_{1 / 2}-54966_{1 / 2}$ & -.02 & -- \\
\hline 6 & 2565. 839 & 38961.92 & & $2177 / 2-6073_{5 / 2}$ & +.04 & -- & $100 \mathrm{R}$ & 2651. 903 & 37697.55 & $(.375,1.105$, & & -.11 & -- \\
\hline $\begin{array}{l}3 \\
2\end{array}$ & $\begin{array}{l}2565.967 \\
2568.817\end{array}$ & $\begin{array}{l}38959.98 \\
38916.75\end{array}$ & & $\begin{array}{l}1507 / 2-54017 / 2 \\
16311 / 2-552211 / 2\end{array}$ & $\begin{array}{l}-.02 \\
+.05\end{array}$ & -- & & & & $\begin{array}{l}1.833) .128, \\
858,1.581\end{array}$ & & & \\
\hline $\begin{array}{l}2 \\
4\end{array}$ & $\begin{array}{l}2568.817 \\
2569.327\end{array}$ & $\begin{array}{l}38916.75 \\
38909.03\end{array}$ & & $\begin{array}{l}103_{11 / 2}-502 \% 11 / 2 \\
117_{9 / 2}-5066_{11 / 2}\end{array}$ & $\begin{array}{l}+.05 \\
+.04\end{array}$ & - & & & & $2.311,3.040$ & & & \\
\hline 10 & 2571. 255 & 38879.87 & $(.332) \mathrm{A}_{-}$ & $115_{5 / 2}-5045_{5 / 2}$ & +.03 & -- & 20 & 2652.911 & 37683.22 & & $150_{7 / 2}-52749 / 2$ & +.11 & -. \\
\hline 1 & 2571. 605 & 38874.58 & & $194_{5 / 2}-5833_{5 / 2}$ & +.05 & .- & $40 \mathrm{R}$ & 2654.120 & 37666.06 & (000) 1.137 . & $115_{5 / 2}-4925_{3} / 2$ & .00 & -- \\
\hline 20 & 2573.760 & 38842.02 & $\begin{array}{c}(.192, .570, \\
947) \quad 633\end{array}$ & $142_{7 / 2}-5305_{5 / 2}$ & +.14 & -- & $\begin{array}{l}10 \\
10\end{array}$ & $\begin{array}{l}2654.773 \\
2655.178\end{array}$ & $\begin{array}{l}37656.79 \\
37651.05\end{array}$ & - & $146_{5 / 2}-522 \gamma_{3 / 2}$ & -.03 & -- \\
\hline & & & $1.012,1.389$ & & & & $\begin{array}{l}10 \\
15\end{array}$ & $\begin{array}{l}2655.178 \\
2655.844\end{array}$ & $\begin{array}{l}37651.05 \\
37641.61\end{array}$ & $(0 \mathrm{w}) 1.186 \mathrm{~h}$ & $\begin{array}{l}138_{3 / 2}-3147_{5 / 2} \\
163_{11 / 2}-5394 / 11 / 2\end{array}$ & $\begin{array}{l}+.02 \\
+.03\end{array}$ & 10 \\
\hline & & & 1.767, 2.144, & & & & $2 \mathrm{~h}$ & 2656.486 & 37632.50 & & $194_{5 / 2}-5709_{7 / 2}$ & $\begin{array}{r}.00 \\
+.10\end{array}$ & -- \\
\hline & 2574.211 & & 2.521 & $1669 / 2-55457 / 2$ & & & & 2656. 950 & 37625.93 & & $247_{7 / 2}-62355_{7 / 2}$ & +.02 & \\
\hline $\begin{array}{l}0 \\
4\end{array}$ & $\begin{array}{l}2574.211 \\
2576.408\end{array}$ & $\begin{array}{l}38835.22 \\
38802.10\end{array}$ & (n) & $\begin{array}{l}1669 / 2-55457 / 2 \\
194_{5 / 2}-58267 / 2\end{array}$ & +.06 & - & 10 & $\begin{array}{l}2657.449 \\
2658.688\end{array}$ & $\begin{array}{l}37618.87 \\
37601.34\end{array}$ & $(318)$ & $217_{7 / 2}-59399 / 2$ & -.02 & -- \\
\hline 6 & 2577.614 & 38783.95 & & $\begin{array}{l}1945 / 2-58207 / 2 \\
197_{1 / 2}-58541 / 2\end{array}$ & $\begin{array}{l}-.04 \\
-.02\end{array}$ & -- & $\begin{array}{r}7 \\
20\end{array}$ & $\begin{array}{l}2658.688 \\
2659.024\end{array}$ & $\begin{array}{l}37601.34 \\
37596.59\end{array}$ & (.318) $1.058 \mathrm{~h}$ & $\begin{array}{l}1643 / 2-5397_{1 / 2} \\
146_{5 / 2}-5221_{5 / 2}\end{array}$ & $\begin{array}{l}-.05 \\
-.06\end{array}$ & $\overline{11}$ \\
\hline 10 & 2579.005 & 38763.04 & (.619) 1.732 & $151_{1 / 2}-5392_{1 / 2}$ & +.03 & & & & & $1.341 \mathrm{~h}$ & & & \\
\hline 7 & 2580. 311 & 38743. 42 & - n......... & $157_{5 / 2}-5451_{7 / 2}$ & .00 & 5 & 10 & 2659.792 & 37585.74 & $(.120)$ & $263_{13 / 2}-6393_{13 / 2}$ & -.06 & -- \\
\hline 9hl & 2581.438 & 38726.50 & & $115_{5 / 2}-5031_{7 / 2}$ & -.24 & -- & 2 & 2660. $\mathrm{C40}$ & 37582.22 & & $231_{5 / 2}-6073_{5 / 2}$ & -.25 & -- \\
\hline 2 & 2582.240 & 38714. 47 & & $146_{5 / 2}-5333_{7 / 2}$ & -.01 & -- & 2 & 2661. 223 & 37565.52 & & $157_{5} / 2-5333_{7 / 2}$ & .00 & -. \\
\hline 3 & 2583. 406 & 38697.00 & $\ldots$ & $157_{5 / 2}-5446_{3 / 2}$ & +.02 & -- & 5 & 2661. 760 & 37557.95 & $\cdots$ & $166_{9 / 2}-5417_{7 / 2}$ & -.02 & -- \\
\hline 4 & 2583.873 & 38690.01 & & $204_{3 / 2}-5917_{5 / 2}$ & +.01 & -- & 4 & 2662. 196 & 37551.80 & & $150_{7 / 2}-5261_{7 / 2}$ & -.02 & \\
\hline 6 & 2584.523 & 38680.29 & & $1507 / 2-5373_{7 / 2}$ & +.02 & & 50 & 2663. 633 & 37531.54 & $(.235, .702$ & $117_{9 / 2}-4928_{11 / 2}$ & -.01 & 12 \\
\hline 10 & 2584.768 & 38676.62 & $\begin{array}{c}(.594) .594 \\
1.304+?\end{array}$ & $151_{1 / 2}-53843 / 2$ & -.02 & 12 & & & & $\begin{array}{l}1.168,1.635, \\
2.103)\end{array}$ & & & \\
\hline 6 & 2585.061 & 38672.22 & & $146_{5 / 2}-5329_{3 / 2}$ & -.04 & -- & 10 & 2664.215 & 37523.35 & (000) $1.307 \ldots$ & $157_{5 / 2}-5329_{3 / 2}$ & +.05 & -8 \\
\hline & 2585. 580 & 38664.46 & & $142_{7 / 2}-5288_{5 / 2}$ & -.02 & -- & 15 & & 37515.02 & $(.272, .818) \ldots$ & $1643 / 2-53843 / 2$ & .00 & $\begin{array}{l}-- \\
--\end{array}$ \\
\hline $50 \mathrm{R}$ & 2586.788 & 38646.41 & $(.704,1.195$, & $117_{9 / 2}-50409 / 2$ & -.08 & -. & 1 & 2666.421 & 37492.30 & & $173_{7 / 2}-5482_{5 / 2}$ & +.10 & - \\
\hline & & & & & & & 20 & 2667.130 & 37482.34 & $(0 w) 1.465$ wC_ & $1737 / 2-5481_{9 / 2}$ & +.04 & -- \\
\hline 6 & $\begin{array}{l}2586.998 \\
2589.594\end{array}$ & $\begin{array}{l}38643.28 \\
38604.53\end{array}$ & $(000) 1$ & $138_{3 / 2}-5246_{1 / 2}$ & -.05 & -- & $\begin{array}{l}2 \\
5\end{array}$ & 2668. 194 & $\begin{array}{l}37467.39 \\
37443.89\end{array}$ & - & $166_{9 / 2}-5408_{7 / 2}$ & .00 & -- \\
\hline 6 & $\begin{array}{l}2009.094 \\
2591.133\end{array}$ & $\begin{array}{l}38604.53 \\
38581.61\end{array}$ & (000) 1.082 & $\begin{array}{l}166_{9 / 2}-5522_{11 / 2} \\
173_{7 / 2}-5591_{9 / 2}\end{array}$ & $\begin{array}{l}-.04 \\
+.01\end{array}$ & $\begin{array}{l}-- \\
--\end{array}$ & $\begin{array}{r}5 \\
20\end{array}$ & $\begin{array}{l}2669.868 \\
2670.236\end{array}$ & $\begin{array}{l}37443.89 \\
37438.74\end{array}$ & $(.316, \quad .542)$ & $\begin{array}{l}115_{5 / 2}-4903_{7 / 2} \\
115_{5 / 2}-4902_{5 / 2}\end{array}$ & $\begin{array}{r}.00 \\
-.07\end{array}$ & -- \\
\hline $30 \mathrm{r}$ & 2591.582 & 38574.93 & $(.373) \mathrm{A}_{-}$ & $115_{5 / 2}-5015_{5 / 2}$ & +.02 & - & 30 & 2670.790 & 37430.97 & $(.672,1.092$ & $142_{7 / 2}-51647 / 2$ & +.01 & -- \\
\hline 10 & 2591.889 & 38570.36 & Whe & $1737 / 2-5589_{7 / 2}$ & -.02 & -- & & & & 1.513) .919, & & & \\
\hline 10 & 2592.843 & 38556.17 & 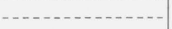 & $117_{9 / 2}-5031_{7 / 2}$ & -.01 & -- & & & & $\mathbf{1 . 3 4 4 ,} 1.769$ & & -02 & \\
\hline $30 \mathrm{R}$ & 2594.854 & 38526.29 & $\cdots$ & $115_{5 / 2}-5011_{5 / 2}$ & -.03 & -- & 3 & 2671.329 & 37423.42 & & $263_{13 / 2}-6377_{13 / 2}$ & -.02 & -- \\
\hline 5 & 2594. 982 & 38524. 39 & & $1427 / 2-527 / 49 / 2$ & -.05 & -- & 40 & 2671.842 & 37416.24 & $(.619,1.022$, & $117_{9 / 2}$ & .00 & -- \\
\hline $60 \mathrm{R}$ & 2595.234 & 38520.65 & & $0_{5 / 2}-38527 / 2$ & -.09 & -- & & & & $1.424,1.826)$ & & & \\
\hline $10 \mathrm{r}$ & 2596.780 & 38497.72 & (000) $1.300 \ldots$ & $157_{5 / 2}-54265 / 2$ & -.04 & -- & & & & $.527, .927$ & & & \\
\hline $\begin{array}{r}50 \mathrm{R} \\
5\end{array}$ & $\begin{array}{l}2599.856 \\
2600.518\end{array}$ & $\begin{array}{l}38452.17 \\
38442.39\end{array}$ & (000) $1.457 \ldots$ & $138_{3 / 2}-5227_{3 / 2}$ & $\begin{array}{l}+.01 \\
-.03\end{array}$ & -- & & & & $\begin{array}{l}1.328,1.728, \\
2.129\end{array}$ & & & \\
\hline 10 & 2600.866 & 38437.24 & $(0 \mathrm{~d} ?) 1.166$ & $\begin{array}{l}179 / 2-50199 / 2 \\
146_{5 / 2}-5305_{5 / 2}\end{array}$ & -.04 & - & 5 & 2672.420 & 37408.15 & & $194_{5 / 2}-5686_{\varepsilon / 2}$ & -.02 & -- \\
\hline 15 & 2601.874 & 38422.36 & & $163_{11 / 2}-5472_{13 / 2}$ & -.01 & -. & $200 \mathrm{R}$ & 2674. 339 & 37381.30 & $(.481,1.440$, & $05 / 2-3738_{3 ! 2}$ & -.11 & \\
\hline 10 & 2602. 926 & 38406.82 & (0d?) $1.200 \ldots$ & $157_{5 / 2}-5417_{7 / 2}$ & -.01 & -- & & & & 2.399). 490 & & & \\
\hline 10 & 2607. 936 & 38333.04 & - & $146_{5 / 2}-52963 / 2$ & +.04 & -- & & & & $1.458,2.423$ & & & \\
\hline 3 & 2608.043 & 38331.47 & 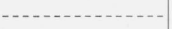 & $150_{7 / 2}-5338_{5 / 2}$ & -.07 & -- & & & & 3.383 & & & \\
\hline 3 & 2608. 746 & 38321.15 & & $150_{7 / 2}-5337_{9 / 2}$ & -.11 & -- & 5 & 2674.917 & 37373. 23 & & $1427 / 2$ & +.01 & -- \\
\hline 5 & 2609.085 & 38316.17 & & $157_{5 / 2}-54087 / 2$ & -.08 & -. & 9 & 2676.088 & & & $138_{3 / 2}-5118_{3 / 2}$ & -.00 & - \\
\hline 5 & 2609. 204 & 38314.42 & - & $224_{3 / 2}-607_{3 / 2}$ & -.03 & -- & 15 & 2677.764 & 37333.49 & (000) $1.281 \mathrm{D} \ldots$ & $146_{5 / 2}-5195_{7 / 2}$ & -07 & -- \\
\hline 9 & 2609. 404 & 38311.49 & & $115_{5 / 2}-4989_{3 / 2}$ & -.12 & -- & 3 & 2678.054 & 37329.45 & & $1669 / 2-539411 / 2$ & .00 & -- \\
\hline $40 R^{4}$ & $\begin{array}{l}2610.690 \\
2611.603\end{array}$ & $\begin{array}{l}38292.60 \\
38279.22\end{array}$ & (000) $.910 \mathrm{C} \dagger[-$ & $\begin{array}{l}197_{1 / 2}-58053 / 2 \\
115_{5 / 2}-49867 / 2\end{array}$ & $\begin{array}{l}-.07 \\
-.04\end{array}$ & $\begin{array}{l}-- \\
--\end{array}$ & 20 & 2679.096 & 37314.93 & $\begin{array}{l}(.459, .784, \\
1.108)\end{array}$ & $150_{7 / 2}-523 \gamma_{i / 2}$ & .00 & -- \\
\hline
\end{tabular}


TABle 2. Classified lines of Re I-Continued

\begin{tabular}{|c|c|c|c|c|c|c|c|c|c|c|c|c|}
\hline Int. & $\lambda_{\mathrm{vac}}$ & $\sigma_{\mathrm{Vac}}$ & Zeeman effect & Transition & $\mathrm{O}-\mathrm{C}$ & Notes & Int. & $\lambda_{\text {vac }}$ & $\sigma_{\mathrm{vac}}$ & Zeeman effect & Transition & $\mathrm{O}-\mathrm{C}$ \\
\hline 30 & $\begin{array}{c}A \\
2679.914\end{array}$ & $\begin{array}{c}K \\
37303,55\end{array}$ & & $151_{1 / 2}-52<61 /$ & & & & $\begin{array}{c}A \\
{ }_{2756}\end{array}$ & 3631266 & & & \\
\hline $\begin{array}{r}30 \\
9\end{array}$ & 2681.010 & 37288. 29 & $\begin{array}{l}(.524) \\
(.331) \\
(.834 \ldots\end{array}$ & $\begin{array}{l}151_{1 / 2}-5246_{1 / 2} \\
157_{5 / 2}-5305_{5 / 2}\end{array}$ & $\begin{array}{l}-.01 \\
-.03\end{array}$ & & $\begin{array}{l}30 \\
20\end{array}$ & $\begin{array}{l}2753.046 \\
2755.212\end{array}$ & $\begin{array}{l}36312.66 \\
36284.12\end{array}$ & $\cdots$ & $\begin{array}{l}146_{5 / 2}-5093_{5 / 2} \\
138_{3}-5011^{2}\end{array}$ & -.06 \\
\hline (2) & 2682. C67 & 37273.60 & & $115_{5 / 2}-4885_{7 / 2}$ & -.04 & 7 & $40 \mathrm{r}$ & 2757.996 & 36247.49 & (0w) $1.401 \mathrm{~h} t$ & $\begin{array}{l}1383 / 2-50115 / 2 \\
146_{5 / 2}-50867 / 2\end{array}$ & $\begin{array}{l}-.04 \\
-.05\end{array}$ \\
\hline 5 & 2682. 368 & 37269.41 & & $1427 / 2-51<8_{9 / 2}$ & -.02 & - & $10 \mathrm{~h}$ & 2758.05 & 36246.7 & (7) 1.7011- & $\begin{array}{l}1405 / 2=0007 / 2 \\
1427 / 2-50465 / 2\end{array}$ & $\begin{array}{l}-.0 n \\
-.2\end{array}$ \\
\hline 3 & 2683. 030 & 37260.22 & & $1427 / 2-5147_{5 / 2}$ & -.07 & - & 10 & 2758. 706 & 36238.17 & & $166_{9 / 2}-52857 / 2$ & +.01 \\
\hline 40 & 2683.558 & 37252.89 & $(0 \mathrm{w}) .959 \mathrm{D}$ & $146_{5 / 2}-5187_{3 / 2}$ & -.08 & - & 20 & 2761.926 & 36195. 92 & (Uw) $1.156 C_{\text {. }}$ & $115_{5 / 2}-477 \gamma_{3 / 2}$ & -.03 \\
\hline 3 & 2684. 382 & 37241.46 & & $194_{5 / 2}-56697 / 2$ & -.06 & -. & 4 & 2762.786 & 36184.66 & & $157_{5 / 2}-51957 / 2$ & +.06 \\
\hline 3 & 2684.905 & 37234.21 & & $221_{9 / 2}-59399_{9 / 2}$ & -.04 & - & 40 & 2763.295 & 36177.99 & $(.118, .342$, & $117_{9 / 2}-4793_{7 / 2}$ & -.04 \\
\hline 100 & 2688.528 & 37184.03 & (0w) $1.456 \mathrm{~B}$ & $157_{5 / 2}-5296_{3 / 2}$ & -.01 & -- & & & & & & \\
\hline $20 \mathrm{H}$ & 2690.247 & 37160.27 & - & $163_{11 / 2}-5346_{11 / 2}$ & -.15 & $-\cdot$ & 40 & 2763.792 & 36171.49 & (.246h) $1.166 \mathrm{~h}$ & $150_{7 / 2}-5122_{7 / 2}$ & +.04 \\
\hline 4 & 2693. 224 & 37119.20 & & $1669 / 2-5373_{7 / 2}$ & +.02 & -. & 9 & 2766.051 & 36141.94 & & $164_{3 / 2}-5246_{1 / 2}$ & .00 \\
\hline 8 & 2693. 719 & 37112.38 & & $151_{1 / 2}-5227_{3 / 2}$ & -.01 & $\cdots$ & 30 & 2766.390 & 36137.52 & & $270_{13 / 2}-6326_{11 / 2}$ & +.03 \\
\hline 8 & 2693.822 & 37110.97 & & $157_{5 / 2}-5288_{5 / 2}$ & +.05 & -- & 7 & 2766.548 & 36135.45 & (000) 1.256... & $150_{7 / 2}-5119_{9 / 2}$ & +.15 \\
\hline 3 & 2694.034 & 37108.03 & & $138_{3 / 2}-509.3_{5 / 2}$ & -.03 & -- & 5 & 2767.576 & 36122.03 & & $1669 / 2-527 / 49 / 2$ & +.01 \\
\hline 20 & 2694.394 & 37103.08 & $\begin{array}{l}(.434, .726 \\
1.018,1.309) \\
.202, .498\end{array}$ & $117_{9 / 2}-48857 / 2$ & .00 & - & 20 & 2767.744 & 36119.84 & $\begin{array}{l}(.095, .283) \\
\mathbf{1 . 0 2 3}, 1.212, \\
1.400\end{array}$ & $115_{5 / 2}-4770_{3 / 2}$ & +.02 \\
\hline 15 & 2695.562 & 37087.00 & $\begin{array}{l}.793,1.092 \\
(000) \\
1.268\end{array}$ & & & & $\underset{15}{6 \mathrm{H}}$ & $\begin{array}{l}2768.282 \\
2768,851\end{array}$ & $\begin{array}{l}36112.81 \\
36105,39\end{array}$ & & $2477 / 2-6083_{5 / 2}$ & -.07 \\
\hline 2 & 2696.159 & 37078.79 & (000) 1.208 & $\begin{array}{l}1575 / 2-52857 / 2 \\
173_{7 / 2}-5440_{5 / 2}\end{array}$ & $\begin{array}{r}-.02 \\
.00\end{array}$ & -- & & & & $\begin{array}{l}(.176, .515, \\
.853,1.192)\end{array}$ & $117_{9 / 2}-4785_{11 / 2}$ & -.02 \\
\hline 7 & 2696.640 & 37072.18 & & $163_{11 / 2}-5337_{9 / 2}$ & -.02 & $\ldots$ & & & & $.337, .000$ & & \\
\hline 20 & 2697.265 & 37063.59 & $\begin{array}{l}(.664) 1.284, \\
2.616\end{array}$ & $0_{5 / 2}-3706_{3 / 2}$ & -.06 & -. & & & & $\begin{array}{l}.337, .675 \\
1.014\end{array}$ & & \\
\hline 3 & 2697.368 & 37062.18 & & $164_{3 / 2}-5338_{5 / 2}$ & -.04 & -- & 3 & 2768.954 & 36104.05 & & $157_{5 / 2}-518 \gamma_{3 / 2}$ & +.04 \\
\hline $15 \mathrm{~h}$ & 2698.794 & 37042.59 & $(.131, .402)$ & $204_{3 / 2}-57525 / 2$ & +.01 & - & $\begin{array}{r}4 \\
70 R\end{array}$ & & $\begin{array}{l}36093.87 \\
36084.99\end{array}$ & & $1427 / 2-50317 / 2$ & +.03 \\
\hline 15 & 2699.580 & 37031.81 & & $117_{9 / 2}-4878_{7 / 2}$ & -.02 & & $\begin{array}{r}70 \mathrm{R} \\
15\end{array}$ & $\begin{array}{l}2770.417 \\
2771.609\end{array}$ & $\begin{array}{l}36084.99 \\
36069.47\end{array}$ & $(0 \mathrm{~W}) 1.021 \mathrm{C}$ & $\begin{array}{l}115_{5 / 2} \text { - } 47666_{7 / 2} \\
138_{3 / 2}-4989_{3 / 2}\end{array}$ & $\begin{array}{l}\overline{-} .06 \\
+.02\end{array}$ \\
\hline $\begin{array}{r}10 \\
3\end{array}$ & 2699. 978 & 37026.35 & & $\begin{array}{l}1179 / 2-40707 / 2 \\
146_{5 / 2}-51647 / 2\end{array}$ & -.01 & -. & $\begin{array}{r}10 \\
8\end{array}$ & 2772.421 & 36058.90 & -n & $\begin{array}{l}1583 / 2-4989_{3 / 2} \\
173_{7 / 2}-5338_{5 / 2}\end{array}$ & $\begin{array}{l}+.02 \\
-.01\end{array}$ \\
\hline 8 & 2700.891 & 37013.84 & ... & $163_{11 / 2}-5332_{9 / 2}$ & +.04 & - & 3 & 2772.687 & 36055.44 & & $172_{1 / 2}-5329_{3 / 2}$ & +.02 \\
\hline 2 & 2700.977 & 37012.66 & $\ldots$ & $1427 / 2-51227 / 2$ & -.12 & -. & 20 & 2773. 109 & 36049.96 & (.578) $1.950 \ldots$ & $172_{1 / 2}-5328_{1 / 2}$ & -.02 \\
\hline 3 & 2704.179 & 36968.83 & & $146_{5 / 2}-5159_{5 / 2}$ & +.21 & -- & 2 & 2773. 214 & 36048.59 & & $1737 / 2-5837_{9 / 2}$ & +.06 \\
\hline 20 & 2704.369 & 36966.23 & $(.191, .572)$ & $164_{3 / 2}-5329_{3 / 2}$ & +.02 & -. & 10 & 2774.383 & 36033.41 & $(.530) \dagger \ldots$ & $138_{3 / 2}-4985_{1 / 2}$ & .00 \\
\hline & & & $\begin{array}{l}\text { 1.147, 1.522, } \\
1.896 \dagger\end{array}$ & & & & $\begin{array}{l}10 \\
10\end{array}$ & $\begin{array}{l}2775.126 \\
2775.126\end{array}$ & $\begin{array}{l}36023.76 \\
36023.76\end{array}$ & . & $\begin{array}{l}273_{11 / 2}-6326_{11 / 2} \\
273_{11 / 2}-6326_{11 / 2}\end{array}$ & $\begin{array}{l}+.01 \\
+.01\end{array}$ \\
\hline 6 & 2704. 769 & 36960.77 & 200 & $164_{1 / 2}-5328_{3 / 2}$ & .00 & -. & 2 & 2775.457 & 36019.47 & & $224_{3 / 2}-58443 / 2$ & -.21 \\
\hline 6 & 2705.076 & 36956.58 & & $146_{5 / 2}-5157_{3 / 2}$ & -.01 & -- & $1 \overline{5}$ & 2775. 639 & 36017.11 & (.457) $1.010_{\ldots}$ & $151_{1 / 2}-5118_{3 / 2}$ & .00 \\
\hline 15 & 2706.056 & 36943.18 & $(.101, .307$ & $150_{7 / 2}-5200_{9 / 2}$ & .00 & -- & 10 & 2775.948 & 36013.10 & - & $247_{7 / 2}-6073_{5 / 2}$ & +.04 \\
\hline 5 & 2706.480 & 36937.40 & $.506, .705)$ & & & & $\begin{array}{l}1 \\
8\end{array}$ & $\begin{array}{l}2776.560 \\
2777.223\end{array}$ & $\begin{array}{l}36005.15 \\
35996.56\end{array}$ & - & $1737 / 2-53337 / 2$ & $\begin{array}{l}+.03 \\
-.03\end{array}$ \\
\hline 10 & 2707.852 & $\begin{array}{l}36918.69 \\
3691\end{array}$ & $(0 \mathrm{w}) .901 \mathrm{~h}$ & $\begin{array}{l}177 / 2-542055 / 2 \\
263_{13 / 2}-6326_{11 / 2}\end{array}$ & $\begin{array}{l}+.04 \\
+.02\end{array}$ & - & $\begin{array}{r}8 \\
15\end{array}$ & $\begin{array}{l}2777.223 \\
2777.71\end{array}$ & $\begin{array}{l}35996.56 \\
35990.3\end{array}$ & & $\begin{array}{l}194_{5 / 2}-5545_{7 / 2} \\
1737 / 2-53329 / 2\end{array}$ & $\begin{array}{l}-.03 \\
+.2\end{array}$ \\
\hline 2 & 2711.382 & 36870.62 & - & $224_{3 / 2}-5929_{3 / 2}$ & .00 & - & 10 & 2778. 498 & 35980.04 & $(.111, .331$ & $142_{7 / 2}-5019_{9 / 2}$ & -.04 \\
\hline 20 & 2712.476 & 36855.76 & $\ldots$ & $146_{5 / 2}-514_{5 / 2}$ & +.07 & -. & & & & $.550) .537, .756$, & & \\
\hline 15 & 2713.024 & 36848.31 & & $166_{9 / 2}-5346_{11 / 2}$ & +.02 & -- & & & & .976 & & \\
\hline 10 & 2713.160 & 36846.47 & - & $1737 / 2-5417_{7 / 2}$ & +.04 & -- & 10 & 2779.082 & 35972.49 & & $231_{5 / 2}-5912_{3 / 2}$ & .00 \\
\hline${ }^{8}$ & 2713.664 & 36839.63 & & $157_{5 / 2}-5261_{7 / 2}$ & +.04 & -- & 15 & 2779. 290 & 35969.79 & & $1507 / 2-51029 / 2$ & +.02 \\
\hline $300 \mathrm{R}$ & 2715.474 & 36815.06 & $\begin{array}{l}(.131, .397 \\
.659, .925\end{array}$ & $117_{9 / 2}-4856_{11 / 2}$ & -.05 & -- & $\begin{array}{r}15 \\
30 \mathrm{r}\end{array}$ & $\begin{array}{l}2780.850 \\
2781.434\end{array}$ & $\begin{array}{l}35949.61 \\
35942.06\end{array}$ & $(.072, .217, .363)$ & $\begin{array}{l}146_{5 / 2}-5057_{3 / 2} \\
142_{7 / 2}-5015_{5 / 2}\end{array}$ & $\begin{array}{l}+.05 \\
+.05\end{array}$ \\
\hline & & & $\begin{array}{r}1.197) .070 \text {, } \\
.336, .603,\end{array}$ & & & & & 2101. T01 & 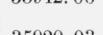 & 1.894 & $1+2 / / 2 \quad-00105 / 2$ & T.00 \\
\hline & & & $\begin{array}{l}.336, .603, \\
.869,1.133\end{array}$ & & & & $\begin{array}{r}7 \\
60\end{array}$ & $\begin{array}{l}2783.141 \\
2783.570\end{array}$ & $\begin{array}{l}35920.03 \\
35914.49\end{array}$ & $(.164, .504, .844$ & $\begin{array}{l}163_{11 / 2}-5222_{13 / 2} \\
117_{9 / 2}-4766_{72}\end{array}$ & $\begin{array}{l}-.02 \\
00\end{array}$ \\
\hline $30 \mathrm{r}$ & 2715.770 & 36811.05 & - & $142_{7 / 2}-5102_{9 / 2}$ & -.05 & -- & & & & $1.184), 2.614$ & & \\
\hline 20 & 2716.246 & 36804.60 & $(280-859$ & $270_{13 / 2}-6393_{13 / 2}$ & -.02 & -. & $50 \mathrm{r}$ & 2785.206 & 35893.39 & $(.085, .256, .427)$ & $142_{7 / 2}-5011_{5 / 2}$ & -.03 \\
\hline $\begin{array}{l}15 \\
(3)\end{array}$ & 2716.754 & $\begin{array}{l}36797.72 \\
36771.05\end{array}$ & $(.280, .859) \ldots$ & $164_{3 / 2}-5312_{3 / 2}$ & +.04 & $=$ & & & & 1.179 & & \\
\hline $\begin{array}{l}(3) \\
15\end{array}$ & & $\begin{array}{l}36771.95 \\
36760.08\end{array}$ & $(442) \mathrm{A}$ & $231_{5 / 2}-59927 / 2$ & -.15 & 7 & 10 & 2785.424 & 35890.58 & & $164_{3 / 2}-5221_{5 / 2}$ & -.02 \\
\hline $\begin{array}{r}15 \\
9\end{array}$ & $\begin{array}{l}2719.536 \\
2719.848\end{array}$ & $\begin{array}{l}36760.08 \\
36755.86\end{array}$ & $(.442) \mathrm{A}=\ldots$ & $\begin{array}{l}166_{9 / 2}-5837_{9 / 2} \\
173_{7 / 2}-5408_{7 / 2}\end{array}$ & +.01 & -- & 9 & 2785. 714 & 35886.85 & $(.689)$ & $172_{1 / 2}-5312_{3 / 2}$ & -.04 \\
\hline 2 & $\begin{array}{l}2719.840 \\
2720.364\end{array}$ & $\begin{array}{l}36755.86 \\
36748.89\end{array}$ & & $\begin{array}{l}173_{7 / 2}-5408_{7 / 2} \\
224_{3 / 2}-5917_{5 / 2}\end{array}$ & +.01 & $-\cdot$ & 15 & 2786.138 & 35881. 39 & $(0 \mathrm{w}) 1.300 \mathrm{~h} \ldots$ & $217_{7 / 2}-57657 / 2$ & -.06 \\
\hline 10 & 2720.659 & 36744.91 & - non & $\begin{array}{l}224_{3 / 2}-5917_{5 / 2} \\
138_{3 / 2}-505 \gamma_{3 / 2}\end{array}$ & $\begin{array}{l}-.01 \\
+.01\end{array}$ & -- & 3 & 2786.450 & 35877.37 & & $157_{5 / 2}-51647 / 2$ & -.03 \\
\hline 7 & 2721.675 & 36731.20 & & $\begin{array}{l}1383 / 2-5057_{3 / 2} \\
164_{3 / 2}-5305_{5 / 2}\end{array}$ & $\begin{array}{l}+.01 \\
-.03\end{array}$ & $\begin{array}{ll}-- \\
-\end{array}$ & $20 \mathrm{r}$ & 2786.558 & 35875.98 & $(.114, .350$, & $150_{7 / 2}-5093_{5 / 2}$ & -.01 \\
\hline 80 & 2722.702 & 36717.35 & $\begin{array}{l}(.100, .286, \\
.470)\end{array}$ & $142_{7 / 2}-5093_{5 / 2}$ & +.03 & -- & & & & $.575) .580$, & & \\
\hline 10 & 2723.356 & 36708.52 & - & $151_{1 / 2}-5187_{3 / 2}$ & -.02 & -. & 7 & 2789.173 & 35842.34 & & $146_{5 / 2}-5046_{5 / 2}$ & .00 \\
\hline 30 & 2723.836 & 36702.05 & & $157_{5 / 2}-5247_{5 / 2}$ & +.16 & - & 15 & 2789. 268 & 35841. 12 & $(334 \mathrm{~h})$ & $138_{3 / 2}-4966_{3 / 2}$ & +.09 \\
\hline $5 \mathrm{~h}$ & 2724.437 & 36693.96 & - & $197_{1 / 2}-5645_{1 / 2}$ & .00 & -- & 30 & 2790.939 & 35819.67 & $(.457, .755)$ & $157_{5 / 2}-5159_{5 / 2}$ & +.01 \\
\hline 9 & 2724.686 & 36690.60 & & $172_{1 / 2}-5392_{1 / 2}$ & .00 & -- & & & & $.566, .867$ & & \\
\hline 3 & 2724. 934 & 36687.27 & & $1737 / 2-5401_{7 / 2}$ & -.10 & - & & & & $1.168,1.468$, & & \\
\hline 20 & 2727.548 & 36652.10 & $\begin{array}{l}(.456, .808, \\
\mathbf{1 . 1 5 9})\end{array}$ & $142_{7 / 2}-5086_{7 / 2}$ & -.04 & -. & $50 \mathrm{r}$ & & & $\begin{array}{l}1.771 \\
(.398) .734 \mathrm{C}\end{array}$ & & \\
\hline $3 \mathrm{~h}$ & 2728. 286 & 36642.19 & & $270_{13 / 2}-637 \gamma_{13 / 2}$ & -.07 & -- & 10 & 2791. 626 & $\begin{array}{l}35815.16 \\
35810.85\end{array}$ & & $\begin{array}{l}163_{11 / 2}-521.1_{13 / 2} \\
150_{7 / 2}-5086_{7 / 2}\end{array}$ & $\begin{array}{r}\overline{7} .04 \\
+.04\end{array}$ \\
\hline 10 & 2728.628 & 36637.60 & $(.172, .496)$ & $138_{3 / 2}-504652$ & -.08 & $\cdots$ & 2 & 2792.940 & 35794.01 & & $263_{13 / 2}-621411 / 2$ & $\begin{array}{r}+.04 \\
.00\end{array}$ \\
\hline 2 & 2729. 209 & 36629.80 & - & $231_{5 / 2}-59787 / 2$ & -.06 & -- & 3 & 2794. 472 & 35774.38 & & $115_{5 / 2}-4735_{7 / 2}$ & -.02 \\
\hline 4 & 2729.427 & 36626.88 & & $164_{3 / 2}-52963 / 2$ & -.07 & -- & 101 & 2796.078 & 35753.84 & & $1669 / 2-5237_{7 / 2}$ & .00 \\
\hline 10 & 2729.635 & 36624.09 & $-\ldots$ & $164_{3 / 2}-5295_{3 / 2}$ & +.01 & -- & 2 & 2796.467 & 35748.87 & & $217_{7 / 2}-5752_{5 / 2}$ & -.04 \\
\hline 10 & 2730.826 & 36608.12 & .......... & $146_{5 / 2}-5122_{7 / 2}$ & -.06 & -- & 9 & 2796.616 & 35746.96 & & $138_{3 / 2}-4957_{5 / 2}$ & -.03 \\
\hline $3 \mathrm{~h}$ & 2731.228 & 36602.73 & -........ & $157_{5 / 2}-5237_{7 / 2}$ & +.03 & -- & 10 & 2798. 104 & 35727.95 & $(0 \mathrm{~W}) 1.385 \mathrm{~B}$ & $173_{7 / 2}-5305_{5 / 2}$ & +.03 \\
\hline & 2731.416 & 36600.20 & $\ldots \ldots$ & $115_{5 / 2}-48187 / 2$ & -.04 & -- & 3 & 2799. 245 & 35713. 39 & (.434) 2.198 & $172_{1 / 2}-5295_{3 / 2}$ & +.10 \\
\hline $50 \mathrm{R}$ & 2732. 206 & 36589.62 & - n.... & $1507 / 2-51647 / 2$ & -.01 & -- & 4 & 2800.746 & 35694. 25 & - & $163_{11 / 2}-5200_{9 / 2}$ & +.03 \\
\hline 10 & 2734.31 & 36561.5 & - n & $146: / 2-5118_{3 / 2}$ & .0 & -- & 3 & 2801. 184 & 35688.66 & & $266_{5 / 2}-6235_{7 / 2}$ & -.04 \\
\hline 10 & 2734.886 & 36553.78 & ........... & $164_{3 / 2}-5288^{i / 2}$ & -.05 & - & 5 & 2804. 506 & 35646.40 & & $142_{7 / 2}-49867 / 2$ & +.04 \\
\hline 6 & 2736.522 & 36531.92 & $\ldots \ldots$ & $150_{7 / 2}-5159_{5 / 2}$ & +.03 & -. & 4 & 2805.105 & 35638. 78 & & $163_{11 / 2}-519411 / 2$ & -.01 \\
\hline 10 & 2736. 767 & 36528.65 & & $273_{11 / 2}-6977_{13 / 2}$ & +.13 & -- & 7 & 2805.977 & 35627.71 & (.496) .987? & $224_{3 / 2}-580.53 / 2$ & -.04 \\
\hline 15 & 2738.323 & 36507.90 & $(0 \mathrm{w}) 1.148 \mathrm{C}$ & $157_{5 / 2}-5227_{3 / 2}$ & +.04 & -- & 20 & 2807.86 & 35603.8 & $(.198,579, .960)$ & $117_{9 / 2}-47357 / 2$ & .0 \\
\hline 4 & 2738.625 & 36503.87 & - n........ & $217_{7 / 2}-5827_{9 / 2}$ & -.04 & -- & 4h & 2808.456 & 35596. 27 & - & $217_{7 / 2}-5737_{7 / 2}$ & -.08 \\
\hline 4 & 2740.074 & 36484.56 & - & $217_{7 / 2}-58267 / 2$ & -.07 & -. & 3 & 2809. 109 & 35587.98 & & $115_{5 / 2}-4717_{3 / 2}$ & -.1 \\
\hline 15 & 2741.967 & 36459.38 & -....... & $221_{9 / 2}-5861_{11 / 2}$ & +.10 & -- & 15 & 2812.073 & 35550.48 & $(.104, .306$, & $173_{7 / 2}-5288_{5 / 2}$ & -.04 \\
\hline 10 & 2742.856 & 36447.57 & - & $157_{5 / 2}-5221_{5 / 2}$ & -.12 & -- & & & & $.509) 1.153$, & & \\
\hline 2 & 2743.122 & 36444.02 & - & $194_{5 / 2}-5590_{3 / 2}$ & -.10 & -- & & & & 1.358, 1.562, & & \\
\hline 3 & 2743.650 & 36437.01 & -....... & $138_{3 / 2}-5026_{3 / 2}$ & -.09 & -- & & & & 1.766 & & \\
\hline 15 & 2743.874 & 36434.04 & - & $163_{11 / 2}-527 / 49 / 2$ & -.11 & $-\cdot$ & 20 & 2812.356 & 35546. 91 & $(.208, .626)$ & $164_{3 / 2}-518 \gamma_{3 / 2}$ & -.01 \\
\hline 20 & 2744. 207 & 36429.62 & - & $117_{9 / 2}-4818_{7 / 2}$ & -.06 & -- & & & & $1.085, \mathbf{1 . 5 0 3 ,}$ & & \\
\hline$\stackrel{2}{5 \mathrm{H}}$ & $\begin{array}{l}2745.014 \\
2745.564\end{array}$ & $\begin{array}{l}36418.91 \\
36411.62\end{array}$ & & $\begin{array}{l}150_{7 / 2}-5147_{5 / 2} \\
244_{5 / 2}-60833_{5 / 2}\end{array}$ & $\begin{array}{l}-.05 \\
-.08\end{array}$ & -- & & 2813.112 & & 1.922 & & \\
\hline 15 & 2745.867 & 36407.60 & (.130) $1.210 \mathrm{~h}$ & $\begin{array}{l}244_{5 / 2}-6083_{5 / 2} \\
173_{7 / 2}-533_{7 / 2}\end{array}$ & $\begin{array}{l}-.08 \\
-.04\end{array}$ & 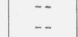 & $\begin{array}{l}15 \\
20 \mathrm{~h}\end{array}$ & $\begin{array}{l}2813.112 \\
2813.964\end{array}$ & $\begin{array}{l}50537.54 \\
35526.59\end{array}$ & & $\begin{array}{l}146_{5 / 2}-5015_{5 / 2} \\
173_{7 / 2}-52857 / 2\end{array}$ & $\begin{array}{l}-.07 \\
-.03\end{array}$ \\
\hline $20 \mathrm{r}$ & 2747.438 & 36386. 79 & $(.291) 1.233 \mathrm{~A}$ & $115_{5 / 2}-4797_{5 / 2}^{\prime 2}$ & -.07 & -. & $40 \mathrm{R}$ & 2814. 676 & 35517.60 & $(.196, .587$ & $\begin{array}{l}173_{7 / 2}-58857 / 2 \\
115_{5 / 2}-4710_{5 / 2}\end{array}$ & -.05 \\
\hline 1 & 2747.627 & 36384.28 & - n- & $204_{3 / 2}-5686_{3 / 2}$ & -.05 & & & & & $.968) .309$ & $1105 / 2-4 / 105 / 2$ & \\
\hline 6 & 2750.322 & 36348.63 & - & $115_{5 / 2}-47937 / 2$ & +.04 & -- & & & & $.695, \mathbf{1 . 0 8 1}$ & & \\
\hline $\begin{array}{r}3 \\
15\end{array}$ & $\begin{array}{l}2751.51 \\
2752.852\end{array}$ & $\begin{array}{l}36333.0 \\
36315.22\end{array}$ & - & $\begin{array}{l}138_{3 / 2}-5015_{5 / 2} \\
115_{5 / 2}-4789_{5 / 2}\end{array}$ & $\begin{array}{l}+.2 \\
-.04\end{array}$ & -. & 10 & 2815,640 & 35505,45 & $1.467,1.855$ & & \\
\hline & & & & & & & & 2010.070 & 50000.40 & & $217_{7 / 2}-57289 / 2$ & -.01 \\
\hline
\end{tabular}


TABLE 2. Classified lines of Re $\mathrm{I}$-Continued

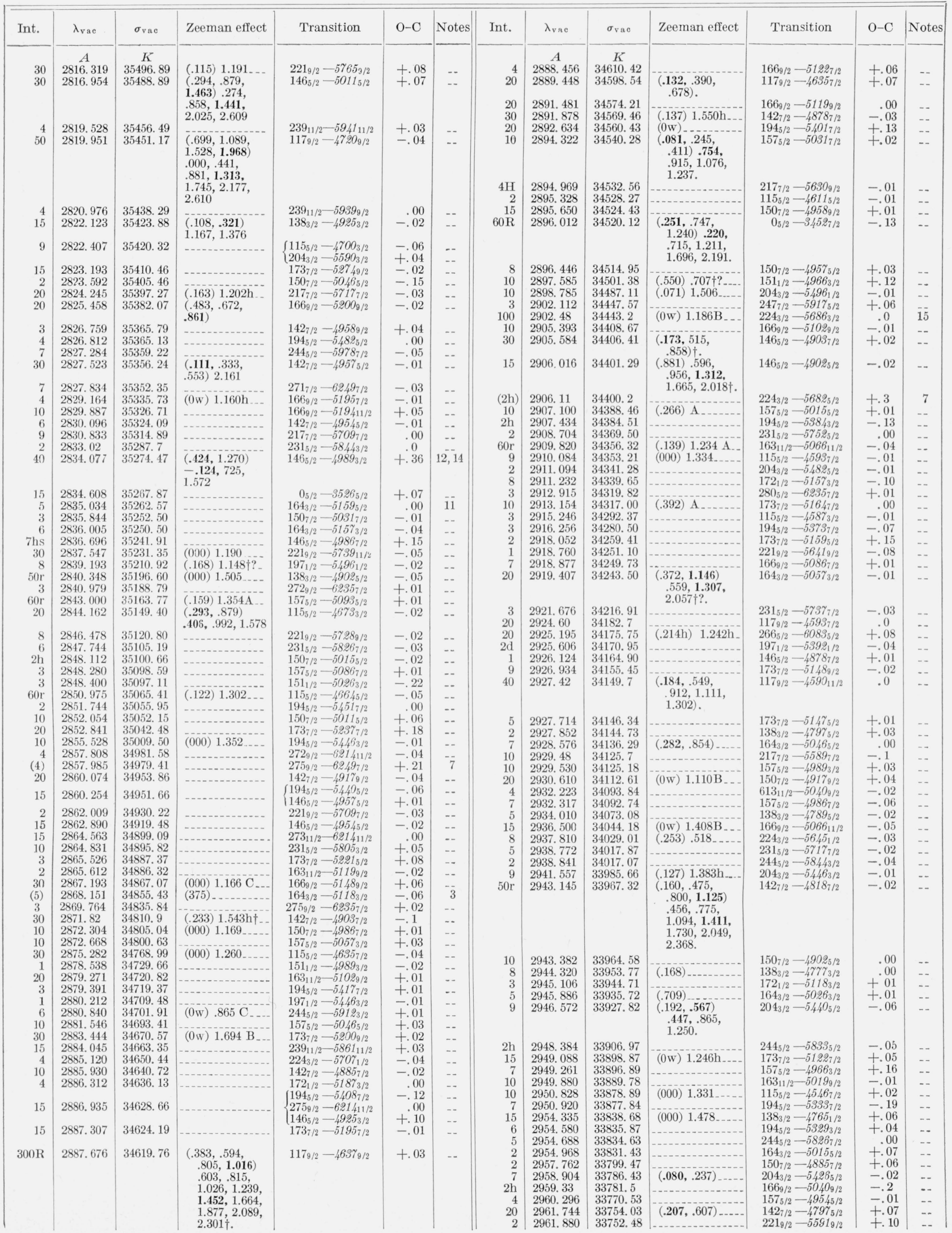


TABLE 2. Classified lines of Re I-Continued

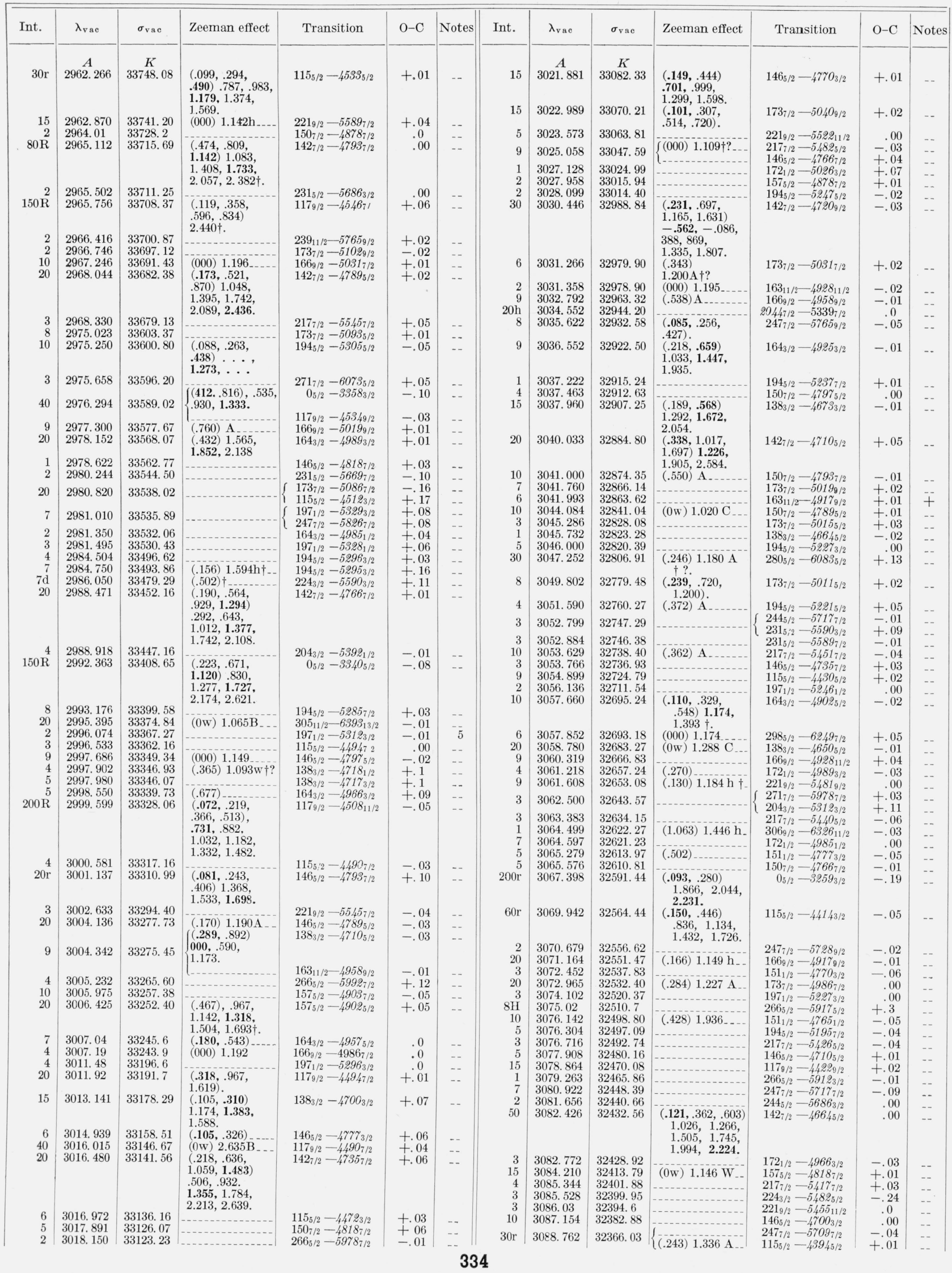


Table 2. Classified lines of Re $\mathrm{I}$-Continued

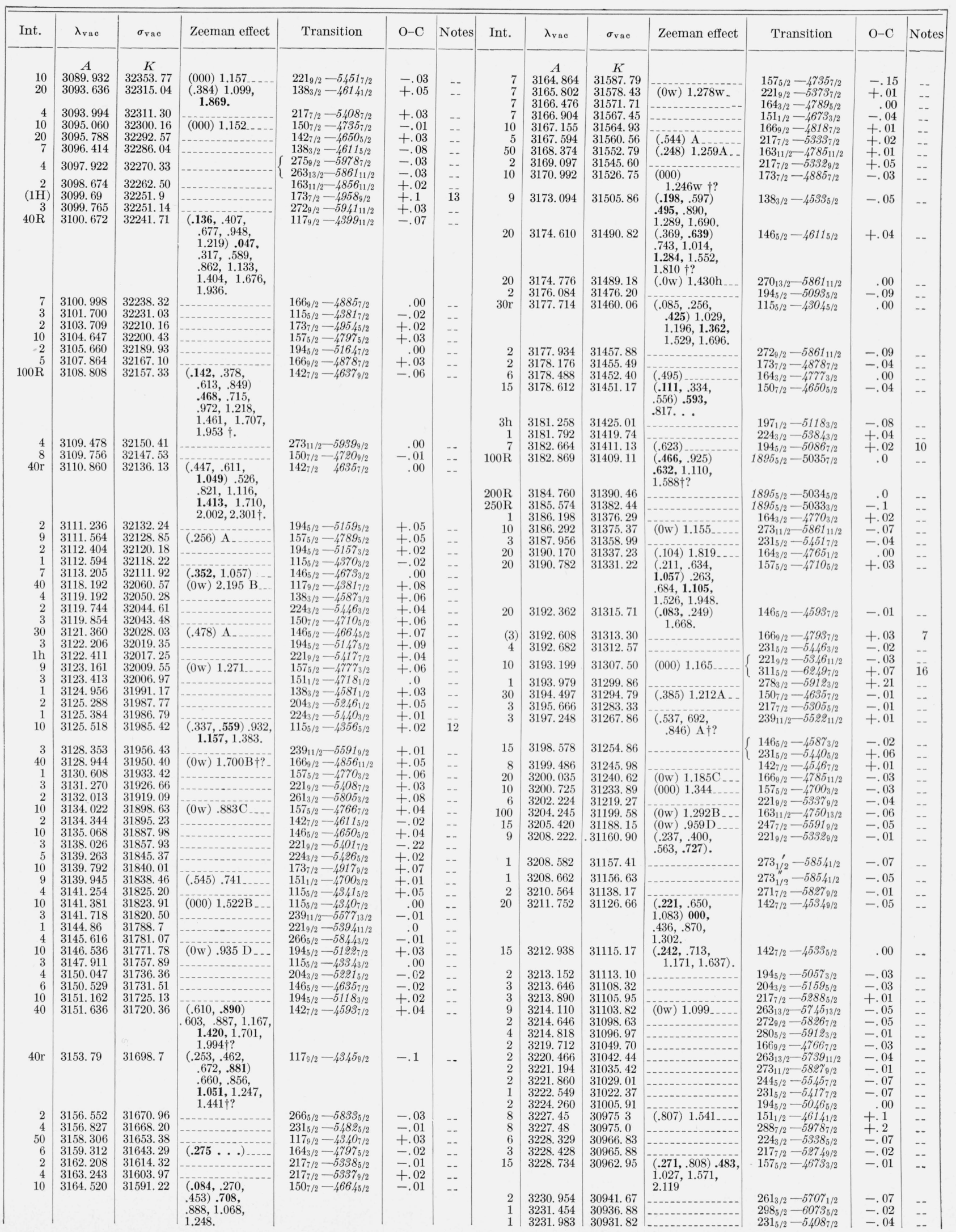


Table 2. Classified lines of Re I-Continued

\begin{tabular}{|c|c|c|c|c|c|c|c|c|c|c|}
\hline Int. & $\lambda_{\mathrm{vac}}$ & $\sigma_{\mathrm{Vac}}$ & Zeeman effect & Transition & $\mathrm{O}-\mathrm{C}$ & Notes & Int. & $\lambda_{\mathrm{vac}}$ & $\sigma_{\mathrm{vac}}$ & Zeeman effect \\
\hline 30 & $\begin{array}{c}A \\
3235.944\end{array}$ & $\begin{array}{c}K \\
30893.96\end{array}$ & $(.077, .239)$ & $138_{3 / 2}-4472_{3 / 2}$ & -.01 & -- & 9 & $\begin{array}{c}A \\
3322.196\end{array}$ & $\begin{array}{c}K \\
30091.91\end{array}$ & \\
\hline & & & $\begin{array}{l}1.235, \mathbf{1 . 4 0 7}, \\
1.571\end{array}$ & $1003 / 2 \quad 4+100 / 2$ & & -- & & & & $(.167, .500$ \\
\hline 10 & 3237.513 & 30878.99 & $\left\{\begin{array}{l}(000) 1.325 \\
(.216, .368, .520)\end{array}\right.$ & $\left\{\begin{array}{l}157_{5 / 2}-4664_{45 / 2} \\
150_{7 / 2}-4593_{7 / 2}\end{array}\right.$ & $\begin{array}{r}-.01 \\
.00\end{array}$ & -- & 50 & 3322.478 & 30089.35 & $\left\{\begin{array}{l}.707,1.041 \\
1.376,1.712,\end{array}\right.$ \\
\hline $\begin{array}{l}4 \\
2\end{array}$ & $\begin{array}{l}3238.363 \\
3238.938\end{array}$ & $\begin{array}{l}30870.88 \\
30865.40\end{array}$ & & $\begin{array}{l}224_{3 / 2}-5329_{3 / 2} \\
224_{3 / 2}-53281 / 2\end{array}$ & $\begin{array}{l}-.01 \\
-.05\end{array}$ & - & & & & $\begin{array}{l}2.048,2.389 \\
2.729 .\end{array}$ \\
\hline $\begin{array}{l}2 \\
4\end{array}$ & $\begin{array}{l}3238.938 \\
3239.156\end{array}$ & $\begin{array}{l}30865.40 \\
30863.33\end{array}$ & $(000) 1.1922 \mathrm{~h}$ & $\begin{array}{l}224_{3 / 2}-53281 / 2 \\
231_{5 / 2}-5401_{7 / 2}\end{array}$ & $\begin{array}{l}-.05 \\
-.05\end{array}$ & -- & & & & \\
\hline 1 & 3239.807 & 30857. 13 & (1000 $1.102010-$ & $239_{11 / 2}-5481_{9 / 2}$ & +.01 & -- & 10 & 3324. 930 & 30067.17 & \\
\hline 4 & 3240.331 & 30852.14 & & $285_{7 / 2}-59399 / 2$ & -.02 & .. & 5 & 3326.896 & 30049.40 & \\
\hline 1 & 3241.023 & 30845.55 & -... & $164_{3 / 2}-4718_{1 / 2}$ & +.2 & -- & 5 & 3327. 204 & 30046.62 & (.276) .521 \\
\hline 1 & 3241.112 & 30844. 71 & & $164_{3 / 2}-4717_{3 / 2}$ & +.1 & -. & 9 & 3327. 712 & 30042.03 & - \\
\hline 10 & 3241.466 & 30841.34 & $(.077, .232, .382)$ & $146_{5 / 2}-4546_{7 / 2}$ & -.03 & - & 4 & 3328. 172 & 30037. 88 & (n) \\
\hline & & & $1.371,1.526$ & & & & $\begin{array}{l}3 \\
2\end{array}$ & 3328.874 & 30031.55 & \\
\hline 2 & 3242.174 & 30834.60 & & $217_{7 / 2}-5261_{7 / 2}$ & -.01 & .- & $\begin{array}{l}2 \\
2\end{array}$ & $\begin{array}{l}3329.319 \\
3330.116\end{array}$ & $\begin{array}{l}30027.53 \\
30020.34\end{array}$ & \\
\hline 10 & 3248.547 & & $(.408,1.221)$ & $164_{3 / 2}-4710_{5 / 2}$ & +.01 & - & 15 & 3331.520 & 30007.69 & $(.157, .471$ \\
\hline 2 & 3251.548 & 30745.71 & & $275_{9 / 2}-5826_{7 / 2}$ & -.01 & - & & & & $.786,1.100)$ \\
\hline 20 & 3252.259 & 30738.99 & & $\begin{array}{l}1669 / 2-47357 / 2 \\
157_{5 / 2}-4650_{5 / 2}\end{array}$ & $\begin{array}{l}-.09 \\
+.01\end{array}$ & -- & & & & $\begin{array}{l}.156, .472, .789, \\
1.105,1.408 .\end{array}$ \\
\hline 2 & 3252.736 & 30734.48 & & $261_{3 / 2}-56866_{3 / 2}$ & -.01 & $\overline{-}$ & 20 & 3335. 364 & 29973.11 & $(.188, .565)$ \\
\hline 10 & 3253. 186 & 30730.23 & & $247_{7 / 2}-5545_{7 / 2}$ & -.03 & $\ldots$ & & & & $.699,1.081$, \\
\hline 2 & 3254.886 & 30714.24 &.- & $\begin{array}{r}278_{3 / 2}-58541 / 2 \\
\left(146_{5 / 2}-4533_{5 / 2}\right.\end{array}$ & $\begin{array}{l}+.01 \\
-.16\end{array}$ & - & & 3337.250 & 29956. 17 & $1.466,1.853 \dagger$ \\
\hline 3 & 3255.292 & 30710.41 & & $\left\{151_{1 / 2}-458 \gamma_{3 / 2}\right.$ & -.04 & - & $300 \mathrm{R}$ & 3338. 178 & 29947. 84 & $(.104, .323$, \\
\hline 8 & 3256.286 & 30700.98 & & $\begin{array}{r}266_{5 / 2}-57_{3} 7_{7 / 2} \\
194_{5 / 2}-5015_{5 / 2}\end{array}$ & $\begin{array}{r}+.09 \\
.00\end{array}$ & - & & & & $\begin{array}{l}.548, .772) \\
.920,1.135+\end{array}$ \\
\hline $\begin{array}{l}0 \\
2\end{array}$ & 3256.666 & 30697. 39 & & $\begin{array}{l}1945 / 2-50105 / 2 \\
221_{9 / 2}-5285_{7 / 2}\end{array}$ & -.01 & $\overline{-}$ & 15 & 3339.685 & 29934. 33 & (.155) 2.361 \\
\hline 7 & 3258.055 & 30684. 30 & & $142_{7 / 2}-4490_{7 / 2}$ & +.01 & - & 3 & 3340.304 & 29928. 78 & \\
\hline $40 \mathrm{r}$ & 3258.848 & 30676.84 & $(.218, .651)$, & $164_{3 / 2}-4700_{3 / 2}$ & +.01 & - & $250 \mathrm{R}$ & $\begin{array}{l}3342.245 \\
3343.092\end{array}$ & $\begin{array}{l}29911.40 \\
29903.83\end{array}$ & $(.376) 1.877 \mathrm{~h} \dagger$ \\
\hline & & & $\begin{array}{l}1.084,1,00 \\
1.928 .\end{array}$ & & & & 100 & $\begin{array}{l}3343.092 \\
3344.322\end{array}$ & $\begin{array}{l}29903.83 \\
29892.82\end{array}$ & $(.156, .484)$ \\
\hline $50 \mathrm{r}$ & 3259.550 & 30670.23 & $\begin{array}{l}(.149, .450), \\
.828,1.127,\end{array}$ & $115_{5 / 2} \quad-4225_{3 / 2}$ & .00 & -- & 40 & & & $\begin{array}{l}1.572 \dagger . \\
(.239, .722)\end{array}$ \\
\hline 1 & 3260.052 & 30665.51 & $1.426,1.726$. & $273_{1 / 2}-5805_{3 / 2}$ & +.13 & & to & 0070.180 & 2.010 .00 & $\begin{array}{l}1.252,1.727, \\
2.199\end{array}$ \\
\hline 20 & 3261.559 & 30651.35 & $(0 \mathrm{~W}) 2.276$ & $151_{1 / 2}-4581_{1 / 2}$ & -.02 & - & 4 & 3346.606 & 29872.43 & \\
\hline 10 & 3262.768 & 30639.98 & $(0 \mathrm{~W}) 1.453 \mathrm{~B}_{\ldots}$ & $1737 / 2-4797_{5 / 2}$ & -.02 & $\ldots$ & 2 & 3348. 292 & 29857.38 & \\
\hline 1 & 3263.877 & 30629.57 & & $285_{7 / 2}-5917_{5 / 2}$ & -.03 & - & 1 & 3348.508 & 29855.46 & \\
\hline 15 & 3266.851 & 30601.69 & (.154) $1.247 \mathrm{~A}$ & $173_{7 / 2}-4793_{7 / 2}$ & -.04 & -. & 8 & 3349.916 & 29842.91 & \\
\hline 15 & 3268. 077 & 30590. 21 & & $138_{3 / 2}-4441_{1 / 2}$ & .00 & -- & o & 3350.094 & 2084133 & \\
\hline 10 & 3268.480 & 30586.44 & (.305) $1.154 \mathrm{~A}_{\text {- }}$ & $1669 / 2-47209 / 2$ & $\begin{array}{r}-.01 \\
-.03\end{array}$ & -- & $\begin{array}{r}4 \\
20\end{array}$ & $\begin{array}{l}3350.094 \\
3353.208\end{array}$ & $\begin{array}{l}29841.33 \\
29813.61\end{array}$ & $(497)$ \\
\hline 1 & $\begin{array}{l}3268.782 \\
3268.894\end{array}$ & $\begin{array}{l}30583.62 \\
30582.57\end{array}$ & (n- & $231_{5 / 2}-5373_{7 / 2}$ & $\begin{array}{r}-.03 \\
.00\end{array}$ & -- & 20 & & & $\begin{array}{l}\text { (.497) 1.203, } \\
2.200 .\end{array}$ \\
\hline $\begin{array}{l}15 \\
15\end{array}$ & $\begin{array}{l}3268.894 \\
3269.033\end{array}$ & $\begin{array}{l}30582.57 \\
30581.27\end{array}$ & & $\begin{array}{l}157_{5 / 2}-46355_{7 / 2} \\
221_{9 / 2}-52749 / 2\end{array}$ & $\begin{array}{r}.00 \\
+.01\end{array}$ & & & & & \\
\hline (1) & 3270.42 & 30568. 3 & - & $173_{7 / 2}-4789_{5 / 2}$ & -.1 & $\overline{13}$ & 30 & 3355.288 & 29795.14 & \\
\hline 2 & 3272. 780 & 30546.26 & & $20447 / 2-5099_{9 / 2}$ & -.1 & -. & 4 & 3355.626 & 29792.14 & \\
\hline 4 & 3273.282 & 30541.57 & & $172_{1 / 2}-47 \gamma \gamma_{3 / 2}$ & -.04 & $\ldots$ & 10 & 3355. 903 & 29789.68 & \\
\hline 1 & 3274.354 & 30531.57 & $\ldots$ & $224_{3 / 2}-52963 / 2$ & -.06 & -- & 10 & 3356. 328 & 29785.90 & \\
\hline 3 & 3274.658 & 30528.73 & & $224_{3 / 2}-5295_{3 / 2}$ & -.03 & - & 9 & 3356.462 & 29784. 71 & $(.149, .452)$ \\
\hline 8 & 3276.060 & 30515.67 & $-\cdots$ & $271_{7 / 2}-5765_{9 / 2}$ & -.05 & $\ldots$ & 4 & 3356.821 & 29781. 52 & \\
\hline 4 & 3276.544 & 30511.17 & - & $266_{5 / 2}-5 \gamma_{1} \gamma_{7 / 2}$ & -.10 & - & 10 & 3358.021 & 29770.88 & $(.180, .539$ \\
\hline 4 & 3277.18 & 30505. 2 & $-\ldots$ & $197_{1 / 2}-5026_{3 / 2}$ & -.1 & -- & & & & $.897)$ 1.077, \\
\hline 20 & 3277. 710 & 30500.31 & & $146_{5 / 2}-4512_{3 / 2}$ & -.04 & $\ldots$ & & & & 1.437, 1.796, \\
\hline 2 & 3281.457 & 30465.48 & $-\cdots$ & $172_{1 / 2}-477 O_{3 / 2}$ & .00 & -- & & & & 2.159 . \\
\hline 4 & 3282.863 & 30452.44 & $\ldots$ & $204_{3 / 2}-5093_{5 / 2}$ & -.01 & -. & 6 & 3358.56 & 29766.1 & \\
\hline 4 & 3283.124 & 30450.02 & (n) & $221_{9 / 2}-5261_{7 / 2}$ & +.05 & -. & $10 \mathrm{~h}$ & 3359.22 & 29760.2 & \\
\hline 5 & 3283.907 & 30442.76 & & $217_{7 / 2}-5221_{5 / 2}$ & +.05 & - & 10 & 3359.818 & 29754. 97 & \\
\hline 3 & 3284.454 & 30437. 69 & & $194_{5 / 2}-4989_{3 / 2}$ & +.01 & .. & 4 & 3360.177 & 29751. 78 & \\
\hline 8 & 3285.638 & 30426.71 & (.513) 2.039 & $172_{1 / 2}-4765_{1 / 2}$ & +.27 & .. & 10 & 3361.145 & 29743. 21 & (000) 1.524 \\
\hline 10 & 3287.127 & 30412.94 & $(000) 1.150 \mathrm{~B}$ & $273_{11 / 2}-5765_{9 / 2}$ & -.03 & - & 3 & 3361.828 & 29737. 17 & \\
\hline 3 & 3287.92 & 30405.6 & (- & $164_{3 / 2}-4673_{3 / 2}$ & -.3 & $\ldots$ & 2 & 3362. 284 & 29733.14 & \\
\hline 6 & 3290.093 & 30385.52 & $-\cdots$ & $117_{9 / 2}-42149 / 2$ & -.02 & .. & 15 & 3362.745 & 29729.07 & (000) $1.280_{-}$ \\
\hline 3 & 3292.088 & 30367.11 & - & $319_{7 / 2}-62357 / 2$ & -.03 & $\ldots$ & 6 & 3363.025 & 29726.59 & \\
\hline 2 & 3292.65 & 30361. 9 & $\begin{array}{l}-1 \\
-2\end{array}$ & $288_{7 / 2}-591 \gamma_{5 / 2}$ & .0 & -. & 9 & 3365. 732 & 29702. 68 & \\
\hline 10 & 3294.834 & 30341.80 & (.229) $1.353 \mathrm{~A}$ & $157_{5 / 2}-4611_{5 / 2}$ & -.02 & - & 8 & 3366. 180 & 29698. 73 & \\
\hline 15 & 3296.696 & 30324.67 & 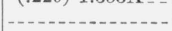 & $146_{5 / 2}-44947 / 2$ & +.01 & -. & 2 & 3366.418 & 29696. 63 & \\
\hline 10 & 3296. 992 & 30321.94 & - & $164_{3 / 2}-46645 / 2$ & +.03 & -. & 3 & 3366.892 & 29692. 45 & \\
\hline 2 & 3299.143 & 30302.17 & & $280_{5 / 2}-5833_{5 / 2}$ & +.07 & .. & 1 & 3367. 298 & 29688.87 & \\
\hline 10 & 3300.972 & 30285. 39 & & $150_{7 / 2}-45349 / 2$ & +.01 & $\ldots$ & 1 & 3367. 384 & 29688.11 & \\
\hline 15 & 3301.595 & 30279.66 & (000) $1.502 \mathrm{~B}_{\ldots}$ & $146_{5 / 2}-4490_{7 / 2}$ & -.03 & .- & 6 & 3367.482 & 29687. 25 & \\
\hline 10 & 3302. 230 & 30273.84 & 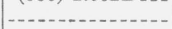 & $150_{7 / 2}-4533_{5 / 2}$ & .00 & .. & 5 & 3367.677 & 29685.53 & (000) 1.023 \\
\hline 6 & 3303.595 & 30261.34 & - & $270_{13 / 2}-5739_{11 / 2}$ & +.04 & $\ldots$ & $2 \mathrm{~h}$ & 3368. 132 & 29681. 51 & \\
\hline 15 & 3303. 754 & 30259.88 & $(000) 1.033 \mathrm{C}$ & $115_{5 / 2}-41847 / 2$ & -.01 & $\ldots$ & 8 & 3368.605 & 29677. 35 & \\
\hline 3 & 3306.484 & 30234.90 & & $231_{5 / 2}-5338_{5 / 2}$ & -.02 &.- & 2 & 3370. 764 & 29658. 34 & \\
\hline 10 & 3307.007 & 30230.12 & $(.064, .192$ & $272_{9 / 2}-5739_{11 / 2}$ & +.03 & - & 8 & 3377. 619 & 29598. 15 & \\
\hline & & & $0, .447, .575)$ & & & & 10 & 3377.743 & 29597.06 & $(.391) 1.251 \mathrm{~A}$ \\
\hline 1 & 3307.454 & 30226.03 & & $217_{7 / 2}-5200_{9 / 2}$ & +.06 & - & 20 & 3379.700 & 29579.93 & \\
\hline 8 & 3308.872 & 30213. 08 & & $221_{9 / 2}-5237_{7 / 2}$ & .00 & -- & 9 & 3381.424 & 29564.84 & \\
\hline 1 & 3309.164 & 30210.41 & & $272_{9 / 2}-5737_{7 / 2}$ & +.01 & -. & 5 & 3381. 794 & 29561. 61 & \\
\hline 7 & 3309.324 & 30208.95 & & $273_{11 / 2}-57 / 45_{13 / 2}$ & .00 & $\ldots$ & 3 & 3382.646 & 29554. 17 & \\
\hline 1 & 3309.795 & 30204.65 & & $266_{5 / 2}-568 \epsilon_{3 / 2}$ & +.02 & .. & 2 & 3383. 259 & 29548.81 & \\
\hline 9 & 3312.288 & 30181.92 & $(.166, .498)$ & $164_{3 / 2}-4650_{3 / 2}$ & +.03 & $\ldots$ & 1 & 3383. 814 & 29543. 96 & \\
\hline & & & $.875,1.210$ & & & & 30 & 3384. 452 & & \\
\hline & & & 1.545 & & & & 40 & 3385.757 & 29527. 01 & $(.214, .644)$ \\
\hline 2 & 3313.105 & 30174.47 & & $263_{13 / 2}-5652_{13 / 2}$ & +.02 & & 2 & 3386. 264 & 29522.59 & \\
\hline 15 & & & (000) 1.274 & $157_{5 / 2}-45937 / 2$ & +.04 & -- & 2 & 3387.053 & 29515.71 & \\
\hline $2 \mathrm{~h}$ & 3314.52 & 30161.6 & - & $266_{5 / 2}-5682_{5 / 2}$ & +.3 & -. & 20 & 3389.428 & 29495. 03 & (.333) 1.528, \\
\hline 6 & 3316.062 & 31047.57 & & $273_{11 / 2}-5739_{11 / 2}$ & +.01 & & & & & \\
\hline 1 & 3316.612 & 30142.58 & & $275_{9 / 2}-5765_{9 / 2}$ & +.04 &.- & 8 & 3389. 767 & 29492.08 & (.141) 1.407 \\
\hline 5 & 3317.013 & 30138. 92 & & $231_{5 / 2}-5329_{3 / 2}$ & +.01 & - & 4 & 3390.032 & 29489.78 & \\
\hline 2 & 3317.152 & 30137. 66 & & $197_{1 / 2}-4989_{3 / 2}$ & .00 & -. & 30 & 3390.254 & 29487.85 & (0w) $1.396 \mathrm{w}_{-}$ \\
\hline 10 & 3318. 671 & 30123.87 & $\ldots$ & $138_{3 / 2}-43945 / 2$ & +.01 & -. & 1 & 3390.970 & 29481. 62 & \\
\hline 1h & 3319. 146 & 30119.56 & & $272_{9 / 2}-57289 / 2$ & +.05 &.- & 1 & 3391. 052 & 29480.90 & \\
\hline 1 & 3319.622 & 30115. 24 & $-\ldots$ & $194_{5 / 2}-495 \gamma_{5 / 2}$ & $\begin{array}{r}.02 \\
+.02\end{array}$ & -. & $5 \mathrm{~h}$ & 3391. 256 & 29479. 13 & \\
\hline 2 & 3320.647 & 30105.95 & & $157_{5 / 2}-4587_{3 / 2}$ & +.03 & - & 8 & 3392.376 & 29469.40 & $(.068, .200$, \\
\hline 1 & 3321.124 & 30101.62 & & $197_{1 / 2}-4985_{1 / 2}$ & .00 & & & & & $.332, .464)$ \\
\hline 6 & 3321.450 & 30098.66 & $\{(.089, .265)$ & $\begin{array}{l}247_{7 / 2}-5482_{5 / 2} \\
146_{5 / 2}-442_{3 / 2}\end{array}$ & $\begin{array}{l}-.14 \\
+.03\end{array}$ & -- & $\begin{array}{l}8 \\
1\end{array}$ & $\begin{array}{l}3394.122 \\
3394.430\end{array}$ & $\begin{array}{l}29454.24 \\
29451.57\end{array}$ & (.296) $1.223 \mathrm{~A} \dagger$ \\
\hline
\end{tabular}


TABLe 2. Classified lines of Re I-Continued

\begin{tabular}{|c|c|c|c|c|c|c|c|c|c|c|c|c|c|}
\hline Int. & $\lambda_{\mathrm{vac}}$ & $\sigma_{\mathrm{vac}}$ & Zeeman effect & Transition & $\mathrm{O}-\mathrm{C}$ & Notes & Int. & $\lambda_{\mathrm{vac}}$ & $\sigma_{\mathrm{vac}}$ & Zeeman effect & Transition & $\mathrm{O}-\mathrm{C}$ & Notes \\
\hline 9 & $\underset{3397.210}{A}$ & $\stackrel{K}{29427.47}^{K}$ & & & & & & 3471023 & $K$ & & & & \\
\hline $\begin{array}{l}9 \\
5\end{array}$ & 3397. 686 & $\begin{array}{l}29427.48 \\
29423.35\end{array}$ & $(0 \mathrm{~W}) \mathrm{D}$ & $\begin{array}{l}263_{13 / 2}-5577_{13 / 2} \\
239_{11 / 2}-5357_{9 / 2}\end{array}$ & $\begin{array}{r}-.08 \\
.00\end{array}$ & -- & $(10 \mathrm{~h})^{6}$ & $\begin{array}{l}3471.923 \\
3471.994\end{array}$ & $\begin{array}{l}28794.23 \\
28793.65\end{array}$ & & $\begin{array}{l}1643 / 2-45123 / 2 \\
275 \%-5630 \% / 2\end{array}$ & $\begin{array}{l}-.07 \\
-.01\end{array}$ & 3 \\
\hline 3 & 3398.404 & 29417. 13 & (0) & $244_{5 / 2}-53843 / 2$ & .00 & -. & 8 & 3472.071 & 28793.01 & & $\begin{array}{l}2 / 59 / 2-50309 / 2 \\
266_{5 / 2}-55457 / 2\end{array}$ & $\begin{array}{l}-.01 \\
-.04\end{array}$ & 3 \\
\hline & 3398.793 & 29413. 77 & & $204_{3 / 2}-4989_{3 / 2}$ & -.07 & .. & 30 & 3472.723 & 28787.60 & & $146_{5 / 2}-4.941_{5 / 2}$ & -.05 & $\begin{array}{l}-- \\
--\end{array}$ \\
\hline $400 \mathrm{R}$ & 3399.302 & 29409.36 & $(.108, .324$ & $117_{9 / 2}-4116_{7 / 2}$ & -.03 & -- & 7 & 3472.875 & 28786.34 & & $146_{5 / 2}-4340_{7} / 2$ & -.07 & - \\
\hline & & & $\begin{array}{l}.539, .769) \\
1.218,1.429\end{array}$ & & & & $\begin{array}{l}2 \\
6\end{array}$ & $\begin{array}{l}3473.000 \\
3473.467\end{array}$ & $\begin{array}{l}28785.30 \\
28781.43\end{array}$ & & $\begin{array}{l}239_{11 / 2}-52749 / 2 \\
1737-4611_{5 / 2}\end{array}$ & $\begin{array}{r}.00 \\
+.01\end{array}$ & - \\
\hline & & & 1.641, 1.853, & & & & 10 & 3474.183 & 28775.50 & & $163_{11 / 2}-4508_{11 / 2}$ & $\begin{array}{r}+.01 \\
+.02\end{array}$ & $\begin{array}{l}-- \\
--\end{array}$ \\
\hline 1 & 3402,97 & & $2.066,2.280$ & $20432-1985$ & & & 5 & 3474.700 & 28771. 22 & & $271_{7 / 2}-5591_{9 / 2}$ & -.07 & -. \\
\hline 1 & 3404.438 & $\begin{array}{l}29377.6 \\
29364.99\end{array}$ & & $\begin{array}{l}2043 / 2-49851 / 2 \\
239_{11 / 2}-5332_{9 / 2}\end{array}$ & $\begin{array}{l}-.2 \\
+.04\end{array}$ & -- & 5 & 3475.201 & 28767.07 & & $\left(\begin{array}{l}3069 / 2-5941_{11 / 2} \\
224_{3 / 2}-5118_{3 / 2}\end{array}\right.$ & $\begin{array}{l}\overline{7} .06 \\
+.04\end{array}$ & -- \\
\hline & & & $\int(.127, .384$ & $115_{5} / 2-40947 / 2$ & -.04 & $\cdots$ & 4 & 3476.031 & 28760.21 & & $\left\{271_{i / 2}-55897 / 2\right.$ & $\begin{array}{l}+.04 \\
+.14\end{array}$ & -- \\
\hline $80 \mathrm{R}$ & 3404.724 & 29362.53 & $\left\{\begin{array}{l}.635) .826 \\
1.119,1.400 \\
1.667,1.933 \\
2.171\end{array}\right.$ & $247_{7 / 2}-5408_{7 / 2}$ & +.08 & & 30 & 3476.442 & 28756.81 & $\begin{array}{l}(.099, .290, \\
.486, .682) .666 \\
.861,1.056, \\
1.251,1.447 \\
1.641,1.835\end{array}$ & $150_{7 / 2}-4381_{7 / 2}$ & -.01 & -. \\
\hline 60 & 3405.888 & 29352.49 & $\begin{array}{l}(0 \mathrm{~W}) \\
((.109, .35 \mathrm{~B}\end{array}$ & $1427 / 2-4356_{5 / 2}$ & -.01 & $\cdots$ & $\begin{array}{l}8 \\
3\end{array}$ & $\begin{array}{l}3477.143 \\
3478.491\end{array}$ & 28751.00 & $(.323) 1.063 \mathrm{~A}$ & $272_{9 / 2}-5591_{9 / 2}$ & -.07 & -- \\
\hline & & & $.580) 1.034$ & $1465 / 2$ & -.01 & & $\begin{array}{l}3 \\
4\end{array}$ & $\begin{array}{l}3478.491 \\
3478.629\end{array}$ & $\begin{array}{l}28739.86 \\
28738.72\end{array}$ & & $\begin{array}{l}2729 / 2-5589_{7 / 2} \\
285_{7 / 2}-57289 / 2\end{array}$ & $\begin{array}{l}+.01 \\
-.01\end{array}$ & -- \\
\hline 30 & 3408.673 & 29328.51 & $\left\{\begin{array}{l}1.270,1.505 \\
1.730\end{array}\right.$ & & & & $\begin{array}{l}30 \\
40\end{array}$ & $\begin{array}{l}3480.381 \\
3480.852\end{array}$ & $\begin{array}{l}28724.26 \\
28720.38\end{array}$ & $\begin{array}{l}(.158) 1.154 \mathrm{~A} \\
(.089, .267)\end{array}$ & $1669 / 2-45349 / 2$ & -.03 & - \\
\hline & & & & $194_{5 / 2}-4878_{7 / 2}$ & +.05 &.- & & & & $871,1.051$ & $1465 / 2-43343 / 2$ & & -- \\
\hline $\begin{array}{r}15 \\
2\end{array}$ & $\begin{array}{l}3409.831 \\
3409.960\end{array}$ & $\begin{array}{l}29318.55 \\
29317.44\end{array}$ & (0W) $1.069 \mathrm{C}_{\ldots} \ldots$ & & $\begin{array}{l}-.05 \\
-.06\end{array}$ & -- & & 3482.233 & 28708.08 & $1.231, \mathbf{1 . 4 1 8}$ & & & \\
\hline 5 & 3412.701 & $\begin{array}{l}29011.44 \\
29293.89\end{array}$ & & $\begin{array}{l}231_{5 / 2}-52475 / 2 \\
247_{7 / 2}-54011_{7 / 2}\end{array}$ & $\begin{array}{l}-.06 \\
-.08\end{array}$ & -- & $\begin{array}{r}40 \\
2\end{array}$ & $\begin{array}{l}3482.233 \\
3482.853\end{array}$ & $\begin{array}{l}28708.98 \\
28703.87\end{array}$ & (0W) $1.208 \mathrm{~W}_{-}-$ & $\begin{array}{r}221_{9 / 2}-50867 / 2 \\
2896\end{array}$ & $\begin{array}{l}+.02 \\
-.01\end{array}$ & -- \\
\hline 10 & 3413.744 & 29284.95 & $(000) \quad 1.178$ & $1669 / 2-4590_{11 / 2}$ & -.02 & - & 5 & 3484.365 & 28691. 42 & $(.261)$ & $261_{3 / 2}-5482_{5 / 2}$ & -.03 & -- \\
\hline 2 & 3414.380 & 29279.49 & & $273_{11 / 2}-5652_{13 / 2}$ & -.04 & -- & 5 & 3484.728 & 28688.43 & (000) 1.138 & $217_{7 / 2}-5046_{5 / 2}$ & +.03 & - \\
\hline 40 & 3417.767 & 29250.48 & $\left\{\begin{array}{l}(0 \mathrm{w}) 1.018 \\
(1.110) 1.265\end{array}\right.$ & $150_{7 / 2}-4430_{5 / 2}$ & -.06 & -- & 4 & 3487.521 & 28665.53 & & $247_{7 / 2}-53388_{5 / 2}$ & +.02 & -- \\
\hline 1 & 3418.330 & 29245.66 & & $\begin{array}{l}151_{1 / 2}-4441_{1 / 2} \\
278_{3 / 2}-5707_{1 / 2}\end{array}$ & $\begin{array}{r}+.04 \\
.00\end{array}$ & -- & $\begin{array}{l}4 \\
5\end{array}$ & $\begin{array}{l}3488.77 \\
3489.850\end{array}$ & $\begin{array}{l}28655.2 \\
28646.32\end{array}$ & & $\begin{array}{l}247_{7 / 2}-533 \gamma_{9 / 2} \\
270_{1}-557 \gamma_{13}\end{array}$ & $\begin{array}{l}+.1 \\
-05\end{array}$ & -- \\
\hline 2 & 3418.933 & 29240.50 & & $266_{5 / 2}-5590_{3 / 2}$ & -.08 & - & 10 & 3490.860 & 28638.04 & $(.577) .813$ & $172_{1 / 2}-458 \gamma_{3 / 2}^{13 / 2}$ & $\begin{array}{r}-.05 \\
.00\end{array}$ & -- \\
\hline 5 & 3419.242 & 29237.85 & & $115_{5 / 2}-4082_{5 / 2}$ & -.02 & -- & 4 & 3491.433 & 28633.34 & & $244_{5 / 2}-5305_{5 / 2}$ & .00 & -- \\
\hline $60 \mathrm{r}$ & 3419.410 & 29236.42 & $(.113, .341$ & $1427 / 2-43459 / 2$ & -.02 & - & 2 & 3491.772 & 28630.56 & & $2857 / 2-571 \gamma_{7 / 2}$ & -.01 & - \\
\hline & & & $\begin{array}{l}.568, .794) \\
.529, .762, .994\end{array}$ & & & & $\begin{array}{l}2 \\
2\end{array}$ & $\begin{array}{l}3492.372 \\
3492.545\end{array}$ & $\begin{array}{l}28625.64 \\
28624.22\end{array}$ & & & +.03 & -- \\
\hline & & & i. $226,1.456$, & & & & 2 & 3494.05 & 28611.8 & & $\begin{array}{l}278_{3 / 2}-5645_{1 / 2} \\
247_{7 / 2}-5333_{7 / 2}\end{array}$ & $\begin{array}{l}.00 \\
+.1\end{array}$ & -. \\
\hline & & & 1.687. & & & & 10 & 3494.725 & 28606.36 & $(.140) 1.280 \mathrm{hw}_{-}$ & $173_{7 / 2}-459.3_{7 / 2}$ & .00 & $\cdots$ \\
\hline $\begin{array}{r}20 \\
5\end{array}$ & $\begin{array}{l}3420.754 \\
3421.582\end{array}$ & $\begin{array}{l}29224.93 \\
29217.86\end{array}$ & (.117) $1.158_{\ldots}$ & $115_{5 / 2}-4080_{3 / 2}$ & +.04 & 17 & 1 & 3495. 415 & 28600.72 & $-1+2+2-10-1$ & $304_{3 / 2}-5912_{3 / 2}$ & +.02 & -- \\
\hline 1h & 3422.923 & $\begin{array}{l}2921.80 \\
29206.42\end{array}$ & - $1.400 \ldots 000)$ & $\begin{array}{r}1383 / 2-43045 / 2 \\
28855 / 2-5806_{7 / 2}\end{array}$ & $\begin{array}{l}-.04 \\
-.04\end{array}$ & -- & $\begin{array}{r}9 \\
40\end{array}$ & $\begin{array}{l}3502.727 \\
3503.056\end{array}$ & $\begin{array}{l}28541.02 \\
28538.34\end{array}$ & $(.234) \quad 1.266 \mathrm{~A}$ & $\begin{array}{l}204_{3 / 2}-4902_{5 / 2} \\
157 / 2-430_{5 / 2}\end{array}$ & $\begin{array}{l}-.02 \\
+.03\end{array}$ & -- \\
\hline $600 \mathrm{R}$ & 3424.620 & 29191.94 & $(000) 1.522$ & $117_{9 / 2}-40947 / 2$ & -.07 & -- & 4 & 3503.305 & 28536.31 & - & $151_{1 / 2}-4370_{3 / 2}$ & .00 & -- \\
\hline & 3425.412 & 29185. 20 & & $275_{9 / 2}-5669_{7 / 2}$ & +.10 & -. & 5 & 3503. 748 & .28532 .70 & & $273_{11 / 2}-557 \gamma_{13 / 2}$ & +.07 & $\because$ \\
\hline $40 \mathrm{r}$ & 3426.189 & 29178.57 & $\begin{array}{l}(.062, .186 \\
.310) .943\end{array}$ & $1737 / 2-4650_{5 / 2}$ & -.01 & -- & $\begin{aligned}(2 \mathrm{~h}) \\
3\end{aligned}$ & $\begin{array}{l}3505.68 \\
3506.177\end{array}$ & $\begin{array}{l}28517.0 \\
28512.93\end{array}$ & & $\begin{array}{l}273^{\prime \prime}{ }_{1 / 2}-5590_{3 / 2} \\
194_{5 / 2}-4797_{5 / 2}\end{array}$ & $\begin{array}{r}+.2 \\
.00\end{array}$ & 7 \\
\hline & & & $1.065,1.186$, & & & & 10 & $\begin{array}{l}3500.168 \\
3506.390\end{array}$ & $\begin{array}{l}28012.93 \\
28511.20\end{array}$ & & $\begin{array}{l}1945 / 2-47975 / 2 \\
224_{3 / 2}-5093_{5 / 2}\end{array}$ & $\begin{array}{r}.00 \\
-.15\end{array}$ & -. \\
\hline 2 & 3426.527 & 29175,69 & $1.311,1.437$. & & -01 & & 10 & 3506.390 & 28511.20\{ & $(.173, .522)$ & $150_{7 / 2}-4356_{5 / 2}$ & +.03 & - \\
\hline 50 & 3427.61 & 29166.5 & $(000) 1.434 \mathrm{D}^{\dagger}$ & $\begin{array}{l}15 / 5 / 2-44947 / 2 \\
150_{7 / 2}-44222_{9 / 2}\end{array}$ & $\begin{array}{l}-.01 \\
+.1\end{array}$ & -- & $\begin{array}{l}3 \\
1\end{array}$ & $\begin{array}{l}3507.334 \\
3508.626\end{array}$ & $\begin{array}{l}28503.53 \\
28493.03\end{array}$ & & $221_{9 / 2}-5066_{11 / 2}$ & +.06 & - \\
\hline 7 & 3428.513 & 29158.80 & $(.125, .378$ & $217_{7 / 2}-5093_{5 / 2}$ & +.02 & - & $\begin{array}{r}1 \\
10\end{array}$ & $\begin{array}{l}3508.626 \\
3510.889\end{array}$ & $\begin{array}{l}28493.03 \\
28474.67\end{array}$ & & $231_{5 / 2}-51647 / 2$ & +.02 & -- \\
\hline & & & $.631)$ & & & & 4 & 3511. 343 & 28470.99 & $(.117, .351)$ & $\begin{array}{l}194_{5 / 2}-4793_{7 / 2} \\
288_{7 / 2}-5728_{9 / 2}\end{array}$ & $\begin{array}{r}+.01 \\
.00\end{array}$ & -- \\
\hline 1 & $\begin{array}{l}3430.44 \\
3430.80\end{array}$ & & & $2805 / 2-5717_{7 / 2}$ & +.1 & -. & 20 & 3512.280 & 28463.38 & $(.105, .320$, & $166_{9 / 2}-4508_{11 / 2}$ & +.03 & -. \\
\hline $\begin{array}{l}1 \\
4\end{array}$ & $\begin{array}{l}3430.80 \\
3431.818\end{array}$ & $\begin{array}{l}29139.4 \\
29130.72\end{array}$ & & $\begin{array}{l}3069 / 2-5978_{7 / 2} \\
1575 / 2-44907 / 2\end{array}$ & $\begin{array}{l}+.1 \\
-01\end{array}$ & -- & 5 & & & $.534, \cdots$ & & & \\
\hline 5 & 3432.668 & 29123.51 & $(.133, .395)$ & $231_{5 / 2}-522 \gamma_{3 / 2}$ & +.04 & -- & $\begin{array}{l}5 \\
5\end{array}$ & $\begin{array}{l}3515.01 \\
3515.756\end{array}$ & $\begin{array}{l}28441.2 \\
28435.25\end{array}$ & (.418) †? & $\begin{array}{l}194_{5 / 2}-4789_{5 / 2} \\
231_{5 / 2}-5159_{5 / 2}\end{array}$ & $\begin{array}{l}-.1 \\
-.02\end{array}$ & -- \\
\hline (3) & 3433.728 & 29114.52 & & $285_{7 / 2}-57659 / 2$ & -.20 & 3 & 5 & 3516.146 & 28432.09 & & $244_{5 / 2}-52855_{7 / 2}$ & +.02 & -. \\
\hline 3 & 3436.193 & 29093.63 & (.310) $1.133 \mathrm{hA}$ & $217_{7 / 2}-5086_{7 / 2}$ & +.03 & $\ldots$ & 40 & 3516.646 & 28428.05 & (.137) $1.522 \mathrm{~A}$ & $138_{3 / 2}-4225_{3 / 2}$ & -.02 & -. \\
\hline 50 & 3437.714 & 29080.76 & $\begin{array}{l}(.409,1.224) \\
-.077, .740\end{array}$ & $1465 / 2-4370_{3 / 2}$ & +.02 & -. & 60 & 3517.326 & 28422.55 & $\begin{array}{c}.146, .439 \\
.732) .704\end{array}$ & $146_{5 / 2}-43045 / 2$ & -.01 & - \\
\hline & & & 1.559, 2.379 & & & & & & & $997,1.290$. & & & \\
\hline 4 & $\begin{array}{l}3438.589 \\
3439.027\end{array}$ & $\begin{array}{l}29073.35 \\
29069.65\end{array}$ & $\ldots$ & $341_{9 / 2}-6326_{11 / 2}$ & -.01 & -- & & & & $1583,1.874$ & & & \\
\hline $\begin{array}{l}2 \\
1\end{array}$ & $\begin{array}{l}3439.027 \\
3439.168\end{array}$ & & $\cdots$ & $221_{9 / 2}-5122_{7 / 2}$ & +.05 & -- & 15 & 3520.723 & 28395. 13 & & $150_{7 / 2}-4345_{9 / 2}$ & +.02 & -- \\
\hline $2 \mathrm{~h}$ & 3439.67 & $\begin{array}{l}29008.40 \\
29064.2\end{array}$ & & $\begin{array}{l}332_{5 / 2}-6.8357 / 2 \\
273_{1 / 2}-5630_{0}\end{array}$ & $\overline{-} .02$ & -- & 1 & & 28392.57 & & $164_{3 / 2}-4472_{3 / 2}$ & -.01 & -. \\
\hline 3 & 3439.776 & 29063.32 & & $\begin{array}{l}273_{11 / 2}-56399_{9 / 2} \\
231_{5 / 2}-5221_{5 / 2}\end{array}$ & +.02 & -- & $\begin{array}{l}4 \\
6\end{array}$ & $\begin{array}{l}3521.746 \\
3522 \\
171\end{array}$ & $\begin{array}{l}28386.88 \\
28383.46\end{array}$ & & $2759 / 2-55897 / 2$ & -.01 & -- \\
\hline $2 \mathrm{~h}$ & 3440.173 & 29059.97 & & $280_{5 / 2}-57097 / 2$ & .00 & -. & $\begin{array}{l}0 \\
4\end{array}$ & $\begin{array}{l}3522.171 \\
3522.528\end{array}$ & $\begin{array}{l}28383.46 \\
28380.58\end{array}$ & & $\begin{array}{l}217_{7 / 2}-5015_{5 / 2} \\
263_{13 / 2}-5472_{13 / 2}\end{array}$ & $\begin{array}{l}\overline{-} .01 \\
+.02\end{array}$ & -- \\
\hline 5 & 3440.843 & 29054.31 & $(.092) \ldots$ & $2242 / 2-514 \gamma_{5 / 2}$ & -.01 & -- & 3 & 3524. 736 & 28362.80 & & $288_{7 / 2}-57_{1} \gamma_{7 / 2}$ & -.03 & -- \\
\hline 4 & 3442.13 & 29043.4 & & $1737 / 2-4637_{9 / 2}$ & .0 & -- & 2 & 3526.21 & 28350.95 & & $150_{7 / 2}-4341_{5 / 2}$ & +.03 & - \\
\hline $5 \mathrm{~h}$ & 3442.722 & 29038.45 & & $278_{3 / 2}-5686_{3 / 2}$ & +.04 & -- & 3 & 3526.368 & 28349.68 & & $150_{7 / 2}-4340_{7 / 2}$ & .00 & - \\
\hline 10 & 3442.967 & 29036.39 & $(.058, .169$, & $163_{11 / 2}-45349 / 2$ & -.03 & -. & 2 & 3527.229 & $\begin{array}{l}28342.76 \\
28334.84\end{array}$ & & $115_{5 / 2}-3992_{3 / 2}$ & -.03 & -. \\
\hline & & & $\begin{array}{l}.279, .350 \\
.500)\end{array}$ & & & & $\begin{array}{r}4 \\
15\end{array}$ & $\begin{array}{l}3528.214 \\
3529.213\end{array}$ & $\begin{array}{l}28334.84 \\
28326.83\end{array}$ & $(.132, .397$, & $\begin{array}{l}217_{7 / 2}-5011_{5 / 2} \\
166 / 2-44947 / 2\end{array}$ & $\begin{array}{l}-.04 \\
-.01\end{array}$ & -- \\
\hline 1 & 3446.743 & 29004.58 & & $164_{3 / 2}-45833_{5 / 2}$ & +.06 & -- & & & & $.663)$ & & -.01 & -- \\
\hline $1 \mathrm{~h}$ & 3447. 801 & 28995.68 & & $301_{1 / 2}-5912_{3 / 2}$ & -.05 & -- & 4 & 3529.74 & 28322.6 & & $231_{5 / 2}-5147_{5 / 2}$ & +.3 & \\
\hline 15 & 3449.333 & 28982.81 & (.397) 1.166, & $151_{1 / 2}--44143 / 2$ & +.25 & -- & 5 & & & & $194_{5 / 2}-4777_{3 / 2}$ & .0 & -- \\
\hline & 3449.407 & 28982.18 & & $285_{7 / 2}-57525 / 2$ & & $\ldots$ & 4 & 3530.893 & 28313.35 & & $271_{7 / 2}-5545_{7 / 2}$ & .00 & - \\
\hline $400 \mathrm{R}$ & 3451.880 & 28961.42 & $(.191, .578)$ & $05 / 2-2896_{3 / 2}$ & -.13 & -- & $2 \mathrm{H}$ & 3532.69 & 28299.0 & & $341_{9 / 2}-62497 / 2$ & -.2 & -- \\
\hline & & & 1.376, 1.761, & & & & $\begin{array}{r}2 \\
20\end{array}$ & $\begin{array}{l}3533.412 \\
3534.822\end{array}$ & $\begin{array}{l}28293.16 \\
28281.88\end{array}$ & $(0 \mathrm{w}) .908 \mathrm{C}$ & $\begin{array}{l}272_{9 / 2}-55457 / 2 \\
1669 / 2-44907 / 2\end{array}$ & $\begin{array}{l}+.03 \\
+.01\end{array}$ & - \\
\hline 15 & 3453 & 2894 & & $157512-1472_{2}$ & +01 & & 1 & $\begin{array}{l}\text { Dost. } 822 \\
3535.30\end{array}$ & 28278.1 & 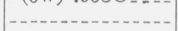 & $\begin{array}{l}1009 / 2-44907 / 2 \\
261_{3 / 2}-5440_{5 / 2}\end{array}$ & $\begin{array}{l}+.01 \\
+.1\end{array}$ & -. \\
\hline 50 & 3453.495 & $\begin{array}{l}20949.00 \\
28947.88\end{array}$ & & $146_{5 / 2}-4.356_{5 / 2}$ & $\begin{array}{l}T .01 \\
-.02\end{array}$ & 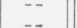 & 5 & 3536.157 & 28271.20 & & $239_{11 / 2}-5222_{13 / 2}$ & .00 & -. \\
\hline & & & $.884) 1.002$ & & & & 50 & 3537.462 & 28260.77 & $(0 \mathrm{~W}) 1.167 \mathrm{~B}_{\ldots}$. & $115_{5 / 2}-39847 / 2$ & -.02 & -- \\
\hline & & & 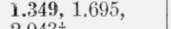 & & & & $\begin{array}{r}2 \\
20\end{array}$ & $\begin{array}{l}3538.784 \\
3539.329\end{array}$ & $\begin{array}{l}28250.22 \\
28245,86\end{array}$ & $(0 \mathrm{~W}) 1.221 \mathrm{C}$ & $\begin{array}{l}298_{5 / 2}-58053 / 2 \\
194,4770_{3 / 2}\end{array}$ & $\begin{array}{l}+.02 \\
-.03\end{array}$ & -- \\
\hline 1 & 3457 & 28910,54 & & & & & 10 & 3539.944 & 28240.96 & & $221_{9 / 2}-5040_{9 / 2}$ & -.01 & -- \\
\hline 15 & 3458.881 & 28902.80 & $(003+3$ & $-16107 / 2$ & .00 & $\cdots$ & 5 & 3543.688 & 28211.12 & & $194_{5 / 2}-47666_{7 / 2}$ & .00 & -. \\
\hline $1000 \mathrm{R}$ & 3460.465 & 28889.57 & $(.903), .360$ & $\begin{array}{r}172_{1 / 2}-46141 / 2 \\
0 \leq 2888 / 2\end{array}$ & $\begin{array}{l}-.01 \\
-.15\end{array}$ & -- & 4 & 3544.370 & 28205.69 & & $263_{13 / 2}-5455_{11 / 2}$ & +.04 & $\ldots$ \\
\hline & & & $.594)$ 1.121, & $05 / 2-2000,7 / 2$ & -.15 & & $\begin{array}{l}3 \\
8\end{array}$ & 3547.024 & $\begin{array}{l}28184.59 \\
28175.99\end{array}$ & & $244_{5 / 2}-5261_{7 / 2}$ & -.02 & -- \\
\hline & & & $1.361,1.599$, & & & & $\begin{array}{l}8 \\
5\end{array}$ & $\begin{array}{l}3548.106 \\
3549.319\end{array}$ & $\begin{array}{l}28175.99 \\
28166.37\end{array}$ & & $\begin{array}{l}151_{1 / 2}-45843 / 2 \\
239_{11 / 2}-5212_{13 / 2}\end{array}$ & $\begin{array}{l}+.03 \\
+.02\end{array}$ & -- \\
\hline & & & $\begin{array}{l}1.831,2.068, \\
2.309 .\end{array}$ & & & & 50 & 3549.891 & 28161.83 & $(.115, .342$, & $117_{9 / 2}-3991_{11 / 2}$ & -.04 & -- \\
\hline 2 & 3463.053 & 28867.98 & & $221_{9 / 2}-5102_{9 / 2}$ & +.06 & -- & & & & $.569, .794$, & & & \\
\hline $7000 \mathrm{R}$ & 3464.726 & 28854.04 & (0w) $1.973 \mathrm{~A} \ldots$ & $05 / 2-2885_{5 / 2}$ & -.14 & -- & & & & $\begin{array}{l}1.050) .282, \\
508, .720\end{array}$ & & & \\
\hline $\begin{array}{r}10 \\
1\end{array}$ & $\begin{array}{l}3465.988 \\
2467.093\end{array}$ & $\begin{array}{l}28843.53 \\
28834.34\end{array}$ & - & $1669 / 2-45.467 / 2$ & $\begin{array}{l}-.02 \\
-.04\end{array}$ & -- & & & & $.928,1.154$ & & & \\
\hline $1 \mathrm{~h}$ & $\begin{array}{l}3467.093 \\
3467.656\end{array}$ & $\begin{array}{l}28834.34 \\
28829.66\end{array}$ & & $\begin{array}{l}305_{11 / 2}-59399_{9 / 2} \\
285_{7 / 2}-5 \gamma^{\prime} \gamma_{7 / 2}\end{array}$ & $\begin{array}{l}\overline{-} .04 \\
+.04\end{array}$ & $\begin{array}{l}-- \\
--\end{array}$ & 2 & 3550.644 & 28155.86 & & $341_{9 / 2}-62357 / 2$ & .00 & 5 \\
\hline 80 & 3467.960 & 28827.14 & $(.071, .212$ & $142_{7 / 2}--43045 / 2$ & -.02 & - & $\begin{array}{r}40 \\
2\end{array}$ & $\begin{array}{l}3551.294 \\
3553.078\end{array}$ & $\begin{array}{l}28150.70 \\
28136.57\end{array}$ & (0w) $1.292 \mathrm{C}$ & $\begin{array}{l}221_{9 / 2}-5031_{7 / 2} \\
261_{3 / 2}-5426_{5 / 2}\end{array}$ & $\begin{array}{l}+.04 \\
-.04\end{array}$ & $\cdots$ \\
\hline
\end{tabular}


Table 2. Classified lines of Re I-Continued

\begin{tabular}{|c|c|c|c|c|c|c|c|c|c|c|c|c|c|}
\hline Int. & $\lambda_{\mathrm{vac}}$ & $\sigma_{\mathrm{vac}}$ & Zeeman effect & Transition & $\mathrm{O}-\mathrm{C}$ & Notes & Int. & $\lambda_{\mathrm{vac}}$ & $\sigma_{\mathrm{vac}}$ & Zeeman effect & Transition & $\mathrm{O}-\mathrm{C}$ & Notes \\
\hline 3 & $\begin{array}{c}A \\
3553.50\end{array}$ & $\begin{array}{c}K \\
28133.2\end{array}$ & & $247_{7 / 2}-5285_{7 / 2}$ & .0 & $\ldots$ & 15 & $\begin{array}{c}A \\
3642.986\end{array}$ & $\begin{array}{c}K \\
27442.19\end{array}$ & $(.068, .201$, & $117_{9 / 2}-3919_{11 / 2}$ & -.03 & -. \\
\hline 20 & 3553. 650 & 28132.04 & (137) $1.249 \mathrm{~A} ?$ & $173_{7 / 2}-45467 / 2$ & +.03 & $\ldots$ & & & & & & & \\
\hline 30 & 3558.935 & 28090.27 & $\begin{array}{l}(.161, .477, \\
.788,1.104)\end{array}$ & $117_{9 / 2}-39847 / 2$ & +.04 & -- & $\begin{array}{l}4 \\
5\end{array}$ & $\begin{array}{l}3643.712 \\
3645.33\end{array}$ & $\begin{array}{l}27436.72 \\
27424.5\end{array}$ & (.185) .948_ & $\begin{array}{l}224_{3 / 2}-4995_{1 / 2} \\
270_{13 / 2}-5455_{11 / 2}\end{array}$ & $\begin{array}{l}+.02 \\
.0\end{array}$ & -- \\
\hline $\begin{array}{l}1 \\
6\end{array}$ & & & - & $217_{7 / 2}-49867 / 2$ & +.04 & -- & 8 & 3645.590 & 27422.58 & $(.617) 1.602$ & $\begin{array}{l}221_{9 / 2}-49589 / 2 \\
197_{12}-4 \gamma 18_{1 / 2}\end{array}$ & +.01 & - \\
\hline $\begin{array}{r}6 \\
10\end{array}$ & $\begin{array}{l}3559.407 \\
3562.454\end{array}$ & $\begin{array}{l}28086.54 \\
28062.52\end{array}$ & & $\begin{array}{l}115_{5 / 2}-3967_{5 / 2} \\
272_{9 / 2}-5522_{11 / 2}\end{array}$ & $\begin{array}{l}-.02 \\
+\quad 02\end{array}$ & -- & 5 & 3646.636 & 27414. 72 & (.617) 1.602 & & $\begin{array}{l}-.3 \\
+.1\end{array}$ & -- \\
\hline 20 & 3564. 732 & 28044.59 & (000) 1.403 & & $\begin{array}{r}+.02 \\
.00\end{array}$ & -- & $\begin{array}{l}1 \\
2\end{array}$ & $\begin{array}{l}3646.69 \\
3649.210\end{array}$ & $\begin{array}{l}27414.3 \\
27395.38\end{array}$ & & $\begin{array}{l}197_{1 / 2}-4717_{3 / 2} \\
217_{7 / 2}-4917_{9 / 2}\end{array}$ & $\begin{array}{l}+.1 \\
+.02\end{array}$ & -- \\
\hline 5 & 3565.195 & 28040.94 & (1)-- & $224_{3 / 2}-5046_{5 / 2}$ & -.03 & - & $\tilde{6}$ & 3649.47 & $\begin{array}{l}27393.4 \\
2730\end{array}$ & & $272_{9 / 2}-5455_{11 / 2}$ & +.1 & -- \\
\hline 1 & 3565.706 & 28036.93 & & $221_{9 / 2}-5019_{9 / 2}$ & +.03 & .. & 20 & 3651.656 & 27377.03 & & $166_{9 / 2}-4399_{11 / 2}$ & +.01 & - \\
\hline 4 & 3566.816 & 28028.20 & & $231_{5 / 2}-5118_{3 / 2}$ & +.01 & -- & 80 & 3651.972 & 27374.67 & $(.127, .384)$ & $164_{3 / 2}-4370_{3 / 2}$ & -.02 & -. \\
\hline $\begin{array}{r}1 \\
30\end{array}$ & $\begin{array}{l}3567.600 \\
3568.234\end{array}$ & 28022.04 & & $\begin{array}{l}197_{1 / 2}-477 \gamma_{3 / 2} \\
2477-52749 / 2\end{array}$ & $\begin{array}{l}+.04 \\
-.02\end{array}$ & -- & & & & $\begin{array}{l}\text { 1.585, 1.835, } \\
2.093 \text {. }\end{array}$ & & & \\
\hline & 3568.234 & 28017.06 & $\begin{array}{l}(.071, .227 \\
.382)\end{array}$ & $24 / 7 / 2-52749 / 2$ & .04 & & $\begin{array}{l}2 \\
2\end{array}$ & $\begin{array}{l}3652.29 \\
3652.563\end{array}$ & $\begin{array}{l}27372.3 \\
27370.23\end{array}$ & & $\begin{array}{l}298_{5 / 2}-57_{11} 7_{7 / 2} \\
2857 / 2-5591_{9 / 2}\end{array}$ & $\begin{aligned} .0 \\
-.06\end{aligned}$ & -- \\
\hline 2 & 3568.780 & 28012.78 & 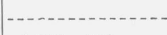 & $\begin{array}{l}288_{7 / 2}-5682_{5 / 2} \\
173_{7 / 2}-45349 / 2\end{array}$ & +.03 & $\cdots$ & 15 & 3653.621 & 27362.31 & & $2363_{9,2}-5099_{9 / 2}$ & -.02 & -- \\
\hline 30 & 3570.255 & 28001.20 & $\begin{array}{c}(.087, .259 \\
431) .997\end{array}$ & $1737 / 2-4533_{5 / 2}$ & -.01 & -- & 4 & 3654.366 & 27356.73 & & $\left\{\begin{array}{l}26655_{12}-5401_{7 / 2} \\
2368_{9 / 2}-5098_{7 / 2}\end{array}\right.$ & $\begin{array}{l}-.03 \\
+.08\end{array}$ & - \\
\hline & & & 1.169, 1.338, & & & & 8 & 3654.935 & 27352.47 & $\begin{array}{l}(.084, .248 \\
.410)\end{array}$ & $2729 / 2-54517 / 2$ & -.02 & -- \\
\hline 2 & $\begin{array}{l}3571.052 \\
3571.694\end{array}$ & $\begin{array}{l}27994.95 \\
27989.92\end{array}$ & $1.000,1000$ & $313_{9 / 2}-5939_{9 / 2}$ & -.04 & -. & 20 & 3660.515 & 27310.77 & (0w) $1.193+?$ & $273_{11 / 2}-5455_{11 / 2}$ & +.04 & -. \\
\hline $\begin{array}{l}8 \\
2\end{array}$ & $\begin{array}{l}3571.694 \\
3572.213\end{array}$ & $\begin{array}{l}27989.92 \\
27985.86\end{array}$ & - & $239_{11 / 2}-519411 / 2$ & +.01 & -- & 10 & 3662.126 & 27298.76 & (.200) $1.171 \mathrm{~A}_{-}$ & $2759 / 2-5 / 4819 / 2$ & $\begin{array}{r}-.05 \\
00\end{array}$ & -- \\
\hline 8 & 3572.804 & 27981. 23 & & $\begin{array}{l}150_{7 / 2}-43045 / 2 \\
164_{3 / 2}-4430_{5 / 2}\end{array}$ & $\begin{array}{l}+.03 \\
+.01\end{array}$ & -- & $\frac{2}{2}$ & $\begin{array}{l}3665.251 \\
3665.522\end{array}$ & $\begin{array}{l}27275.49 \\
27273.47\end{array}$ & & $\begin{array}{l}1945 / 2-40 / 3 / 2 \\
1575 / 2-43045 / 2\end{array}$ & $\begin{array}{r}.00 \\
-.13\end{array}$ & -- \\
\hline 2 & 3572.961 & 27980.00 & & $273_{11 / 2}-5522_{11 / 2}$ & $\begin{array}{l}+.01 \\
+.01\end{array}$ & -- & 3 & 3669.159 & 27246.44 & & $197_{1 / 2}-4700_{3 / 2}$ & +.01 & $\begin{array}{l}-- \\
--\end{array}$ \\
\hline 3 & 3573.730 & 27973.98 & & $3069_{/ 2}-5861_{11 / 2}$ & -.01 & - & 8 & 3669.436 & 27244.38 & $(.748) \ldots$ & $224_{3 / 2}-4956_{3,2}$ & -.04 & - \\
\hline $\begin{array}{l}1 \\
6\end{array}$ & $\begin{array}{l}3577.09 \\
3577.325\end{array}$ & 27947.7 & $(233)$ & $244_{5 / 2}-523 \gamma_{7 / 2}$ & .0 & -. & 40 & 3669.778 & 27241.81 & $(.103, .309)$ & $164_{3 / 2}-4356_{5 / 2}$ & -.01 & -- \\
\hline $\begin{array}{l}6 \\
1\end{array}$ & $\begin{array}{l}3577.325 \\
3577.923\end{array}$ & $\begin{array}{l}27945.87 \\
27941.20\end{array}$ & $(.233)--$ & $\begin{array}{l}197_{1 / 2}-47 \gamma 0_{3 / 2} \\
311_{5 / 2}-5912_{3 / 2}\end{array}$ & $\begin{array}{r}.00 \\
-.02\end{array}$ & - & 7 & 3669.993 & 27240.25 & $1.190,1.396$. & $117_{0 / 2}-3899_{9 / 2}$ & +.04 & $\ldots$ \\
\hline 2 & 3578.054 & 27940.17 & & $275_{9 / 2}-55457 / 2$ & .00 & - & 10 & 3670. 358 & 27237.54 & & $239_{11 / 2}-5119_{9 / 2}$ & $\begin{array}{r}.05 \\
+.05\end{array}$ & -. \\
\hline 60 & 3579.125 & 27931.81 & $\begin{array}{r}(.322, .973) \\
.330, .990,\end{array}$ & $157_{5 / 2}-43 \% 0_{3 / 2}$ & +.03 & .. & 50 & 3670.528 & 27236.28 & $\begin{array}{l}(.108, .314 \\
.521, .728)\end{array}$ & $142_{7} / 2-4145_{9 / 2}$ & -.04 & - \\
\hline $\begin{array}{l}50 \\
80\end{array}$ & 3580.970 & 27917.42 & $\begin{array}{l}1.643 \\
\text { (0w) } 1.226\end{array}$ & $163_{11 / 2}-4422_{9 / 2}$ & -.01 & - & & & & $\begin{array}{l}\mathbf{6 3 5}, .842, \\
1.049,1.258 .\end{array}$ & & & \\
\hline $\begin{array}{r}80 \\
7\end{array}$ & $\begin{array}{l}3583.02 \\
3583.146\end{array}$ & $\begin{array}{l}27901.5 \\
27900.47\end{array}$ & (.303) $1.488 \mathrm{~A}$ & $\begin{array}{l}117_{9 / 2}-39659 / 2 \\
194_{5 / 2}-4735_{7 / 2}\end{array}$ & $\begin{array}{l}.0 \\
00\end{array}$ & - & (1) & 3671.24 & 27231.0 & & $\begin{array}{l}247_{7 / 2}-51957 / 2 \\
244_{72}-51647 / 2\end{array}$ & $\begin{array}{l}+.2 \\
-08\end{array}$ & 13 \\
\hline & 5000.140 & 28000.46 & . $825,1.041$ & $1945 / 2-4 / 357 / 2$ & .00 & -- & 20 & 3672.407 & 27222.34 & $\left\{\begin{array}{l}(0 \mathrm{w}) 1.314 \mathrm{~B} . \\
.\end{array}\right.$ & $\begin{array}{l}244_{5} / 2-51647 / 2 \\
146_{5} / 2-41847 / 2\end{array}$ & $\begin{array}{l}-.08 \\
-.05\end{array}$ & $\begin{array}{l}-- \\
--\end{array}$ \\
\hline $\begin{array}{r}15 \\
10 \mathrm{~d}\end{array}$ & $\begin{array}{l}3585.030 \\
3585.329\end{array}$ & $\begin{array}{l}27885.81 \\
27883.48\end{array}$ & $(.927) .252$ & $\begin{array}{l}247_{7 / 2}-5261_{7 / 2} \\
172_{1 / 2}-4512_{3 / 2}\end{array}$ & $\begin{array}{l}+.02 \\
-.03\end{array}$ & -. & 15 & 3676.000 & 27195.73 & $(.088, .265$ & $166_{9} / 2-4381_{7 / 2}$ & .00 & - \\
\hline 1 & & 27871.72 & $.565^{\circ}$ & $1821 / 2-40163 / 2$ & & & & & & $\begin{array}{l}.443, .620) \\
.555, .733 .\end{array}$ & & & \\
\hline 2 & 3589. 264 & 27852.91 & 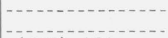 & $\begin{array}{l}280_{5 / 2}-5590_{3 / 2} \\
244_{5 / 2}-522 \gamma_{3 / 2}\end{array}$ & $\begin{array}{l}+.03 \\
+.03\end{array}$ & -- & $\begin{array}{l}4 \\
2\end{array}$ & $\begin{array}{l}3676.570 \\
3677.722\end{array}$ & $\begin{array}{l}27191.52 \\
27183.00\end{array}$ & (.053) $1.359 \mathrm{~h}_{-}$ & $\begin{array}{l}195_{5} / 2-4664_{5}-2 \\
204^{\prime 2}-4765_{1 / 2}\end{array}$ & -.01 & -- \\
\hline 10 & 3590.878 & 27840.39 & (.240) .532, & $224_{3 / 2}-5026_{3 / 2}$ & .00 & 19 & 6 & 3678.392 & $\begin{array}{l}27183.00 \\
27178.05\end{array}$ & & $\begin{array}{l}204_{3} / 2-4765_{1 / 2} \\
172_{1} / 2-4441_{1 / 2}\end{array}$ & $\begin{array}{l}-.01 \\
+.02\end{array}$ & -- \\
\hline 1 & 3591.86 & 27832.7 & 1.010 & $314_{3 / 2}-59299_{3 / 2}$ & & & 10 & 2630.206 & $2,164.65$ & $(.117) 1.114 \mathrm{~A}$ & $244_{5} / 2-5159_{5 / 2}$ & $\begin{array}{l}+.02 \\
-.03\end{array}$ & -- \\
\hline 10 & 3593.388 & 27820.95 & & $\begin{array}{l}314_{3 / 2}-59299_{3 / 2} \\
164_{3 / 2}-44143 / 2\end{array}$ & $\begin{array}{l}\overline{7} .1 \\
+.01\end{array}$ & - & 8 & 3681. 284 & 27156.70 & $(.360) .29$ & $261_{3} / 2-5328_{1 / 2}$ & -.01 & -- \\
\hline 15 & 3595.164 & 27807.20 & $(0 \mathrm{~W}) 1.507 \mathrm{C}$ & $217_{7 / 2}-49589 / 2$ & $\begin{array}{l}+.01 \\
-.01\end{array}$ & -- & $\begin{array}{l}2 \\
4\end{array}$ & $\begin{array}{l}3681.407 \\
3681.834\end{array}$ & $\begin{array}{l}27155.79 \\
27152,64\end{array}$ & & & -.10 & -- \\
\hline $2 \mathrm{~h}$ & 3595.32 & 27806.0 & & $266_{5 / 2}-5446_{3 / 2}$ & .0 & -- & $\begin{array}{l}4 \\
5\end{array}$ & $\begin{array}{l}3681.834 \\
3682.155\end{array}$ & $\begin{array}{l}27152.64 \\
27150.28\end{array}$ & & $\begin{array}{l}244_{5} / 2-515 \gamma_{3} / 2 \\
224_{3} / 2-495 \gamma_{5 / 2}\end{array}$ & $\begin{array}{r}-.01 \\
.00\end{array}$ & $\begin{array}{l}-- \\
--\end{array}$ \\
\hline 2 & 3595.887 & 27801.61 & & $304_{3 / 2}-5833_{5 / 2}$ & +.2 & -. & 8 & 3682.714 & 27146.16 & & $163^{1 / 2}-4345_{9 / 2}$ & +.01 & -- \\
\hline 10 & 3596.230 & 27798.96 & & $\begin{array}{l}3197 / 2-59787 / 2 \\
1575 / 2-43565 / 2\end{array}$ & -.07 & -- & 1 & 3685.303 & 27127.08 & & $271_{7} / 2-5426_{5 / 2}$ & +.03 & - \\
\hline 20 & 3596.392 & 27797.71 & $(.106,315$ & $\begin{array}{l}15 / 5 / 2-43065 / 2 \\
217_{7 / 2}-495 \gamma_{5 / 2}\end{array}$ & $\begin{array}{r}+.02 \\
.00\end{array}$ & -- & $\begin{array}{l}2 \\
3\end{array}$ & $\begin{array}{l}3685.444 \\
3686.452\end{array}$ & $\begin{array}{l}27126.05 \\
27118.63\end{array}$ & -..- & $\begin{array}{l}221_{9 / 2}-4928_{11 / 2} \\
2631_{3 / 2}-5346_{11 / 2}\end{array}$ & $\begin{array}{r}+.02 \\
+.02\end{array}$ & - \\
\hline & & & $\begin{array}{l}.531) \text {.621, } \\
.831,1.041\end{array}$ & & & & 6 & $\begin{array}{l}3686.452 \\
3688.646\end{array}$ & 27102.50 & & $\begin{array}{l}2031^{1 / 2}-5591_{9 / 2} \\
288_{7} / 2\end{array}$ & $\begin{array}{l}+.02 \\
-.1\end{array}$ & -- \\
\hline 3 & 3597.039 & 27792.71 & 1.245 & & & & 150 & 3689.504 & 27096.20 & $\begin{array}{l}.1177, .439 \\
.732) 1.144\end{array}$ & $142_{7} / 2-4131_{5 / 2}$ & +.04 & 12 \\
\hline 15 & 3598.767 & 27779.36 & $(.313, .517)$ & $\begin{array}{l}244_{5 / 2}-5221_{5 / 2} \\
231_{5 / 2}-5093_{5 / 2}\end{array}$ & $\begin{array}{l}.00 \\
-.01\end{array}$ & -- & & & & $\begin{array}{l}\text { 1.429, 1.715, } \\
\text { 1.991. }\end{array}$ & & & \\
\hline & & & $\begin{array}{l}.877,1.081 \\
1.286,1.490\end{array}$ & & & & $\begin{array}{r}(1) \\
1\end{array}$ & $\begin{array}{l}3690.16 \\
3690.547\end{array}$ & $\begin{array}{l}27091.4 \\
27088.54\end{array}$ & & $\begin{array}{l}288_{7} / 2-558.9_{7 / 2} \\
151_{1} / 2-42255_{3 / 2}\end{array}$ & $\begin{array}{l}+.1 \\
+.24\end{array}$ & 13 \\
\hline 15 & 3604,394 & 27736.00 & $\begin{array}{l}1.694 . \\
(.326,978)\end{array}$ & 224 & & & 20 & 3691.385 & 27082. 39 & & $273_{1 / 2}^{\prime \prime}-5446_{3 / 2}$ & $\begin{array}{r}+19 \\
+\end{array}$ & -- \\
\hline 15 & 3604.394 & -1600.00 & $1.101,1.752$, & $224_{3 / 2}$ & -.04 & -. & & 360148 & 27081.7 & & $\begin{array}{r}217_{7 / 2}-48857 / 2 \\
150_{7 / 2}-4214^{9} / 2\end{array}$ & $\begin{array}{l}+.19 \\
-.2\end{array}$ & - \\
\hline & 3605.971 & 27723.87 & 2.404 & & & & 200 & 3691.48 & $2,081.7$ & & $1643 / 2-4341_{5 / 2}$ & +.1 & - \\
\hline 4 & 3606. 558 & 27719.35 & $\ldots \ldots \ldots$ & $\begin{array}{l}298_{5 / 2}-57 \tilde{\delta} 2_{5 / 2} \\
305_{11 / 2}-58 \% \gamma_{9 / 2}\end{array}$ & -.06 & -- & 10 & $\begin{array}{l}3692.115 \\
3692.808\end{array}$ & 27077. 03 & & $266_{5 / 2}-5.373_{7 / 2}$ & .00 & -. \\
\hline 10 & 3607.232 & 27714.18 & (0w) 1.325 & $\left\{\begin{array}{l}305_{11 / 2}-5827^{9 / 2} \\
231_{5 / 2}-5086_{\pi / 2}\end{array}\right.$ & $\begin{array}{l}-.05 \\
-.01\end{array}$ & -- & $\begin{array}{l}2 \\
2\end{array}$ & $\begin{array}{l}3692.808 \\
3693.664\end{array}$ & $\begin{array}{l}27071.95 \\
27065.68\end{array}$ & & $\begin{array}{l}239_{11 / 2}-5102_{9 / 2} \\
298_{5 / 2}-5686_{3 / 2}\end{array}$ & -.01 & -- \\
\hline 2 & 3607.833 & 27709.56 & (0w) $1.325 \ldots$ & $\left\{\begin{array}{l}194_{5 / 2}-47_{1} 7_{3 / 2}^{1 / 2} \\
\end{array}\right.$ & -.0 & $\cdots$ & 3 & 3695.600 & 27051.50 & & $\begin{array}{l}298_{5 / 2}-56863 / 2 \\
194_{5 / 2}-4650_{5 / 2}\end{array}$ & -.01 & -- \\
\hline 10 & 3608.661 & 27703.20 & & $2759 / 2-5522_{11 / 2}$ & +.02 & -. & 2 & 3697.124 & 27040.35 & & $275_{9 / 2}-5455_{11 / 2}$ & +.05 & - \\
\hline 30 & 3610.492 & 27689.15 & & $221_{9 / 2}-4986_{7 / 2}$ & +.02 & - & 20 & 3697.710 & 27936.07 & & $271_{7 / 2}-5411_{7 / 2}$ & -.05 & - \\
\hline 2 & 3611.437 & 27681.91 & $(0 \mathrm{~W}) 1.234 \mathrm{~A}$ & $163_{11 / 2}-4899_{11 / 2}$ & $\begin{array}{r}.00 \\
+.09\end{array}$ & -- & $2 \mathrm{~d}$ & $\begin{array}{l}3700.684 \\
3701.184\end{array}$ & $\begin{array}{l}27014.34 \\
27010.69\end{array}$ & 6000 & & $\begin{array}{r}.00 \\
-.03\end{array}$ & -- \\
\hline$(2 \mathrm{H})$ & 3613.41 & 27666.8 & ser & $\begin{array}{l}271_{7 / 2}-5482_{5 / 2} \\
314_{3 / 2}-5912_{3 / 2}\end{array}$ & $\begin{array}{l}+.02 \\
+.1\end{array}$ & 7 & $\begin{array}{l}4 \\
5\end{array}$ & $\begin{array}{l}3701.184 \\
3702.098\end{array}$ & $\begin{array}{l}27010.69 \\
27004.02\end{array}$ & $\begin{array}{l}(000) 1.188 \\
(.370, .613)\end{array}$ & $\begin{array}{l}221_{9 / 2}-4.917_{9 / 2} \\
231_{5 / 2}-5015_{5 / 2}\end{array}$ & -.04 & - \\
\hline 8 & 3615.372 & 27651.78 & -- & $272_{9 / 2}-5481_{9 / 2}$ & +.01 & -. & $(2 \mathrm{~h})$ & 3702.74 & 26999.4 & & $275_{9 / 2}-5451_{7 / 2}$ & -.1 & 7 \\
\hline 3 & 3616.427 & 27643.72 & & $194_{5 / 2}-4710_{5 / 2}$ & .00 & & 100 & 3703.243 & 26995,67 & $(.180, .540)$ & $138_{3 / 2}-4082_{5 / 2}$ & -.04 & -- \\
\hline 80 & 3617.085 & 27638.68 & $\begin{array}{l}(.777) 1.103, \\
1.315,1.526^{\dagger} .\end{array}$ & $157_{5 / 2}-4341_{5 / 2}$ & -.01 & 19 & & & & $\begin{array}{l}.588, .947, \\
1.306,1.665 \text {. }\end{array}$ & & & \\
\hline 20 & 3617. 252 & 27637.41 & & $157_{5 / 2}-4340_{7 / 2}$ & -.04 & .. & 1 & 3703.53 & 26993.6 & & $261_{3 / 2}-5312_{3 / 2}$ & .0 & -- \\
\hline 20 & 3617.859 & 27632.77 & $(.209, .621) \ldots$ & $146_{5 / 2}-4225_{3 / 2}$ & +.04 & -- & 10 & & & & $138_{3 / 2}-4 G 81_{3 / 2}$ &.$- \mathrm{C3}$ & -- \\
\hline 1 & 3619.203 & 27622.51 & 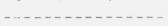 & $1643 / 2-43945 / 2$ & +.04 & $\ldots$ & 20 & 3705.02 & 26982. & & $138_{3 / 2}-408 O_{1 / 2}$ & +.1 & -- \\
\hline 2 & 3620.145 & 27615.33 & & $173_{7 / 2}-4 / 4947 / 2$ & +.03 & -- & 8 & 3705.686 & & & $1737 / 2-4430_{5 / 2}$ & -.03 & -- \\
\hline 2 & 3620.230 & 27614.68 & & $3069 / 2-58267 / 2$ & -.02 & -- & $6 \mathrm{~h}$ & 3708. 774 & 26955.42 & & $231_{5 / 2}-5011_{5 / 2}$ & -.05 & -- \\
\hline 30 & 3621.460 & 27605.29 & $(.328,1.205) \mathrm{A}_{\text {. }}$ & $1669 / 2-44229 / 2$ & -.01 & -- & 60 & 3709.930 & 26947.02 & $(.382, .629$, & $142_{7 / 2}-4116_{7 / 2}$ & -.03 & -- \\
\hline 10 & 3622.183 & 27599.78 & - & $263_{13 / 2}-599411 / 2$ & $\begin{array}{l}+.01 \\
\end{array}$ & -- & & & & $.875) .698$ & & & \\
\hline 2 & 3622.265 & 27599.16 & $((.167, .509)$ & $\begin{array}{l}270_{13 / 2}-5472_{13 / 2} \\
1575 / 2-43343 / 2\end{array}$ & $\begin{array}{l}-.22 \\
-.00\end{array}$ & -- & & & & $\begin{array}{l}.948,1.198, \\
1.447,1.697,\end{array}$ & & & \\
\hline 20 & 3625.908 & 27571.43 & $\left\{\begin{array}{l}1.154,1.489, \\
\mathbf{1 . 8 2 3} \dagger\end{array}\right.$ & $10 / 5 / 2-40343 / 2$ & & & 2 & 3710.64 & 26941.8 & $1.947,2.196$ & $301_{1 / 2}-570 \gamma_{1 / 2}$ & +.1 & -. \\
\hline & & & & $298_{5 / 2}-5 \gamma_{3} \gamma_{7 / 2}$ & +.06 & -- & 5 & & & $(.258) \ldots$ & & + . & $-\cdot$ \\
\hline 8 & 3626.053 & 27570.33 & (000) 1.242 & $1737 / 2-44907 / 2$ & .00 & -. & $5 \mathrm{~h}$ & 3712.920 & 26925. 32 & & $272_{9 / 2}-54087 / 2$ & $\begin{array}{r}.00 \\
-02\end{array}$ & - \\
\hline 20 & 3629.200 & 27546.42 & $(0 \mathrm{w}) 1.455 \mathrm{~B}$ & $194_{5 / 2}-4700_{3 / 2}$ & -.03 & -. & 10 & 3713.160 & 26923.58 & & $247_{7 / 2}-51647 / 2$ & -.02 & -- \\
\hline 2 & 3632.154 & 27524.02 & & $304_{3 / 2}-5805_{3 / 2}$ & +.04 & -. & 1 & 3714.530 & 26913. 64 & & $338_{7 / 2}-6073_{5 / 2}$ & -.10 & -- \\
\hline 4 & 3636.772 & 27489.07 & $(.135, .396)_{-}$ & $2043 / 2-4797_{5 / 2}$ & -.02 & -. & 1 & 3714. 712 & 26912,32 & & $285_{7 / 2}-5545_{7 / 2}$ & -.03 & -- \\
\hline 40 & 3637.064 & 27486.86 & $\begin{array}{l}(.102, .304) \\
.969,1.175\end{array}$ & $148_{3 / 2}-4131_{5 / 2}$ & -.04 & - & 5 & 3714. 995 & 26910.28 & $\begin{array}{l}(.479) 1.088 \\
2.040 \text {. }\end{array}$ & $172_{1 / 2}-44143 / 2$ & +.13 & -- \\
\hline & & & 1.379 & & -0 & & 100 & $\begin{array}{l}3717.285 \\
3717.44\end{array}$ & $\begin{array}{l}26893.70 \\
26892.6\end{array}$ & (000) $1.257 \mathrm{~W}_{\ldots} \ldots$ & $\begin{array}{l}173_{7 / 2}-4422 \sigma_{6} \\
305\end{array}$ & $\begin{array}{l}-.06 \\
-.2\end{array}$ & -- \\
\hline 10 & $\begin{array}{l}\text { 3637. } 228 \\
3637.732\end{array}$ & $\begin{array}{l}27485.62 \\
27481.82\end{array}$ & & $273_{11 / 2}-5472013 / 2$ & $\begin{array}{l}-.02 \\
+.03\end{array}$ & -- & $\begin{array}{l}20 \\
2 \mathrm{~h}\end{array}$ & $\begin{array}{l}3717.44 \\
3720.860\end{array}$ & $\begin{array}{l}20892.0 \\
26867.86\end{array}$ & & $221_{9 / 2}-4903_{7 / 2}$ & +.05 & $\cdots$ \\
\hline $\begin{array}{l}15 \\
60\end{array}$ & $\begin{array}{l}3637.732 \\
3637.848\end{array}$ & $\begin{array}{l}27481.82 \\
27480.94\end{array}$ & $\begin{array}{l}(.601) \\
\text { (.171) } 1.304 \mathrm{~A}\end{array}$ & $\begin{array}{l}172_{1 / 2}-447 \%_{3 / 2} \\
115_{5 / 2}-3906_{5 / 2}\end{array}$ & $\begin{array}{l}T .00 \\
-.02\end{array}$ & -. & 4 & $\begin{array}{l}5720.000 \\
3721.146\end{array}$ & 26865.79 & & $2477 / 2-5159_{5 / 2}$ & -.07 & $\cdots$ \\
\hline $\begin{array}{r}00 \\
2\end{array}$ & $\begin{array}{l}3637.848 \\
3641.202\end{array}$ & 27455.63 & & $332_{5} / 2-6073_{5 / 2}$ & .00 & -. & (3) & 3722.22 & 26858.1 & & $324_{5 / 2}-5929_{3 / 2}$ & -.2 & 13 \\
\hline
\end{tabular}


Table 2. Classified lines of Re $\mathrm{I}$-Continued

\begin{tabular}{|c|c|c|c|c|c|c|c|c|c|c|c|c|c|}
\hline Int. & $\lambda_{\mathrm{vac}}$ & $\sigma_{\mathrm{Vac}}$ & Zeeman effect & Transition & $\mathrm{O}-\mathrm{C}$ & Notes & Int. & $\lambda_{\mathrm{vac}}$ & $\sigma_{\mathrm{vac}}$ & Zeeman effect & Transition & $\mathrm{O}-\mathrm{C}$ & Notes \\
\hline $2000 \mathrm{R}$ & $\begin{array}{c}A \\
3725.760\end{array}$ & $\begin{array}{c}K \\
26832.52\end{array}$ & $(.073, .218$ & $2363_{9 / 2}-5047_{11 / 2}$ & .00 & 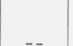 & 20 & $\begin{array}{c}A \\
3829.80\end{array}$ & $\begin{array}{c}K \\
26103.6\end{array}$ & (.776) .196_ & $172_{1 / 2}-43343 / 2$ & & $\ldots$ \\
\hline & 3796502 & $26827 \quad 18$ & $\begin{array}{l}.373, .413, \\
.653) .981 \ldots\end{array}$ & & & & $\begin{array}{r}10 \\
2\end{array}$ & $\begin{array}{l}3829.83 \\
3830.098\end{array}$ & $\begin{array}{l}26103.4 \\
26101.58\end{array}$ & & $\begin{array}{l}338_{7 / 2}-5992_{7 / 2} \\
298_{5 / 2}-5590_{3 / 2}\end{array}$ & $\begin{array}{l}+.1 \\
-.05 \\
+.04\end{array}$ & 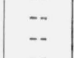 \\
\hline $\begin{array}{l}8 \\
4\end{array}$ & $\begin{array}{l}3726.502 \\
3726.68\end{array}$ & $\begin{array}{l}26827.18 \\
26825.9\end{array}$ & & $\begin{array}{l}224_{3 / 2}-49255_{3 / 2} \\
151_{1 / 2}-4199_{1 / 2}\end{array}$ & $\begin{array}{l}-.01 \\
+.2\end{array}$ & -- & & 3831.028 & & & $231_{5 / 2}-49255_{3 / 2}$ & & \\
\hline 7 & 3727.498 & 26820.01 & $(.056) .704$ & $261_{3 / 2}-52955_{3 / 2}$ & -.01 & - & & & 2607704 & (.343) 1.033 & $273_{11 / 2}-5332_{9 / 2}$ & -.03 & -- \\
\hline $1 \mathrm{~h}$ & 3729. 684 & 26804. 29 & & $244_{5 / 2}-51227 / 2$ & +.05 & & 70 & 3833.702 & 26077.04 & $1.183,1.333$, & $1737 / 2-4340_{7 / 2}$ & -.01 & -- \\
\hline 20 & 3731.870 & 26788.60 & $\begin{array}{l}(.118, .557) \\
.809 \dagger .\end{array}$ & $166_{9 / 2}-4340_{7 / 2}$ & +.01 & 19 & 100 & 3834.235 & 26073.42 & $\begin{array}{l}1.488,1.652 \dagger ? \\
(.059, .177,\end{array}$ & $157_{5 / 2}-41847 / 2$ & -.01 & -- \\
\hline $\begin{array}{r}(5 \mathrm{H}) \\
40\end{array}$ & $\begin{array}{l}3732.058 \\
3732.278\end{array}$ & $\begin{array}{l}26787.25 \\
26785.67\end{array}$ & & $\begin{array}{l}272_{9 / 2}-539411 / 2 \\
1507-41847 / 2\end{array}$ & -.13 & 7 & & & & $.296) .894$ & & & \\
\hline 20 & 3735.01 & 26766.1 & $(.152 \mathrm{~W}) 1.176 \mathrm{~A}_{-}$ & $\begin{array}{l}150_{7 / 2}-41847 / 2 \\
117_{9 / 2}-38527 / 2\end{array}$ & $\begin{array}{l}+.01 \\
-.1\end{array}$ & -- & & & & $\begin{array}{l}\text { 1.024, 1.145, } \\
1.264 .\end{array}$ & & & \\
\hline 1000 & 3735.314 & 26763.90 & (.413) $1.712 \mathrm{~A}_{-}$ & $2363_{9 / 2}-5039_{9 / 2}$ & .00 & $\cdots$ & 70 & 3836.305 & 26059.35 & . 1.587 & $197_{1 / 2}-4581_{1 / 2}$ & .00 & $-\infty$ \\
\hline $4 \mathrm{~h}$ & 3736.193 & 26757. 60 & - & $244_{5 / 2}-5118_{3 / 2}$ & .00 & -. & $1 \mathrm{~h}$ & 3837.088 & 26054.03 & & $3069 / 2-56697 / 2$ & -.05 & -- \\
\hline 10 & 3736.838 & 26752.98 & $\ldots$ & $247_{7 / 2}-5147_{5 / 2}$ & +.05 & - & 10 & 3841.498 & 26024.12 & (0w) $1.123 \mathrm{~h} \ldots$ & $221_{9 / 2}-48187 / 2$ & -.04 & -- \\
\hline 1 & 3737.806 & 26746.05 & $\ldots$ & $3069_{/ 2}-5739_{11 / 2}$ & -.06 & -- & 60 & 3843.415 & 26011.15 & $(.097, .292 \ldots)$ & $117_{9 / 2}-37 \gamma_{67 / 2}$ & +.02 & $\overline{13}$ \\
\hline 1 & 3738.538 & 26740.82 & & $231_{5 / 2}-4989_{3 / 2}$ & +.06 & & (3) & 3844.35 & 26004.8 & 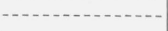 & $194_{5 / 2}-45467 / 2$ & -.1 & 13 \\
\hline $\begin{array}{r}200 \\
30\end{array}$ & $\begin{array}{l}3740.10 \\
3740.414\end{array}$ & $\begin{array}{l}26729.7 \\
26727.41\end{array}$ & $(0 \mathrm{w}) 1.485 \mathrm{C}$ & & $\begin{array}{l}.0 \\
.00\end{array}$ & 19 & $\begin{array}{r}7 \\
15\end{array}$ & $\begin{array}{l}3846.857 \\
3851.988\end{array}$ & $\begin{array}{l}25987.87 \\
25953.26\end{array}$ & $(0 \mathrm{w}) 1.459 \mathrm{~B}$ & $\begin{array}{l}280_{5 / 2}-54017 / 2 \\
275_{9 / 2}-5346_{11 / 2}\end{array}$ & $\begin{array}{l}.00 \\
.00\end{array}$ & -- \\
\hline $\begin{array}{r}30 \\
2\end{array}$ & $\begin{array}{l}3740.414 \\
3743.072\end{array}$ & $\begin{array}{l}26727.41 . \\
26708.43\end{array}$ & & $\begin{array}{r}20009 / 2-00507 / 2 \\
231_{5 / 2}-49867 / 2\end{array}$ & $\begin{array}{r}.00 \\
+.02\end{array}$ & $\cdots$ & $\begin{array}{r}10 \\
5\end{array}$ & 3852.689 & 25948.53 & . & $266_{5 / 2}-5261_{7 / 2}$ & -.05 & -- \\
\hline 1 & 3743.194 & 26707.56 & & $239_{11 / 2}-5066_{11 / 2}$ & +.05 & - & 30 & 3855.936 & 25926.69 & $(.202) 1.512$, & $164_{3 / 2}-4225_{3 / 2}$ & +.01 & -- \\
\hline 2 & 3743.572 & 26704.86 & & $273_{11 / 2}-539411 / 2$ & +.01 & -- & & & & 1.643 & & & \\
\hline 80 & 3745.436 & 26691.57 & $\begin{array}{l}(000) \text { 1.065, } \\
\mathbf{1 . 2 0 6}, 1.341,\end{array}$ & $146_{5 / 2}-4131_{5 / 2}$ & +.01 & -- & 1 & 3858.584 & 25908.89 & & $\left\{\begin{array}{l}273^{\prime}{ }_{1 / 2}-53299_{3 / 2} \\
273^{\prime \prime}{ }_{1 / 2}-5329_{3 / 2}\end{array}\right.$ & $\begin{array}{l}-.43 \\
+.37\end{array}$ & $\begin{array}{l}-- \\
--\end{array}$ \\
\hline & & & 1.472 . & & & & 2 & 3859. 328 & 25903.90 & & & +.02 & -- \\
\hline 5 & $\begin{array}{l}3750.666 \\
3752718\end{array}$ & & $\cdots$ & $194_{5 / 2}-4611_{5 / 2}$ & .00 & -- & 2 & & $\begin{array}{l}25903.08 \\
25893.60\end{array}$ & & $\begin{array}{r}273^{1 / 2}-53288_{1 / 2} \\
217^{\prime 2}-4766_{7 / 2}\end{array}$ & $\begin{array}{r}.00 \\
-\quad 01\end{array}$ & -- \\
\hline 2 & & $\begin{array}{l}26639.77 \\
26635.53\end{array}$ & & $278_{3 / 2}-54463 / 2$ & $\begin{array}{r}+.02 \\
.00\end{array}$ & -- & $\begin{array}{l}9 \\
6\end{array}$ & $\begin{array}{l}3860.862 \\
3861.647\end{array}$ & $\begin{array}{l}20890.00 \\
25888.34\end{array}$ & & $\begin{array}{l}2177 / 2-47667 / 2 \\
1507 / 2-40947 / 2\end{array}$ & $\begin{array}{r}-.01 \\
.00\end{array}$ & -- \\
\hline $\begin{array}{l}1 \\
2\end{array}$ & $\begin{array}{l}3753.316 \\
3753.770\end{array}$ & $\begin{array}{l}20000.50 \\
26632.31\end{array}$ & & $\begin{array}{l}3069 / 2-5728_{9 / 2} \\
266_{5 / 2}-5329_{3 / 2}\end{array}$ & $\begin{array}{r}.00 \\
+.02\end{array}$ & $\begin{array}{ll}-- \\
-\end{array}$ & $\begin{array}{l}6 \\
6\end{array}$ & 3862.103 & 25885.28 & & $244_{5 / 2}-5031_{7 / 2}$ & -.02 & - \\
\hline 15 & 3755.624 & 26619.17 & $(.063, .188$ & $173_{7 / 2}-43945 / 2$ & +.01 & -- & 5 & 3863.153 & 25878.25 & & $263_{13 / 2}-5222_{13 / 2}$ & +.01 & -- \\
\hline & & & $\begin{array}{l}.313) \mathbf{. 9 4 5}, \\
1.069,1.194\end{array}$ & & & & $\begin{array}{r}15 \\
4\end{array}$ & $\begin{array}{l}3863.768 \\
3863.932\end{array}$ & $\begin{array}{l}25874.13 \\
25873.04\end{array}$ & & $\begin{array}{l}194_{5 / 2}-4533_{5 / 2} \\
231_{5 / 2}-4903_{7 / 2}\end{array}$ & $\begin{array}{r}-.01 \\
.00\end{array}$ & -- \\
\hline 15 & 3757.622 & 26605.01 & $(.223, .668)$ & $142_{7 / 2}-4082_{5 / 2}$ & +.04 & -- & 4 & 3864. 766 & 25867.45 & & $2857 / 2-5440_{5 / 2}$ & -.03 & - \\
\hline 2 & 3759.749 & 26589.96 & & $314_{3 / 2}-58053 / 2$ & .00 & -- & 1 & 3865.126 & 25865.04 & & $275_{9 / 2}-5337_{9 / 2}$ & .00 & - \\
\hline 2 & 3765.878 & 26546.68 & & $304_{3 / 2}-5 \gamma_{0 \gamma_{1 / 2}}$ & -.03 & -. & $2 \mathrm{~h}$ & 3865.875 & 25860.03 & & $3340_{5 / 2}-5926_{5 / 2}$ & -.03 & -. \\
\hline 15 & 3766.480 & 26542.44 & $(.082, .247$ & $146_{5 / 2}-4116_{7 / 2}$ & -.01 & -- & 2 & 3867.21 & 25851.1 & & $305_{11 / 2}-5641_{19 / 2}$ & -.2 & - \\
\hline & & & $\begin{array}{l}1.576,1.740 . \\
1.5764\end{array}$ & & & & 100 & $\begin{array}{l}3868.213 \\
3869.944\end{array}$ & $\begin{array}{l}25844.33 \\
25832.84\end{array}$ & $(0 \mathrm{w}) 1.306 \mathrm{C}$ & $\begin{array}{l}1003 / 2-401 / 5 / 2 \\
163_{11 / 2}-42149 / 2\end{array}$ & -.07 & $\begin{array}{l}-- \\
-\end{array}$ \\
\hline $5 \mathrm{~d}$ & 3769.297 & 26522.60 & $(.249) \ldots \ldots$ & $204_{3 / 2}-4700_{3 / 2}$ & -.01 & -- & 3 & 3873.037 & 25812.21 & & $280_{5 / 2}-53843 / 2$ & .00 & - \\
\hline 8 & 3770.738 & 26512.47 & & $231_{5 / 2}-4966_{3 / 2}$ & +.03 & -- & 4 & 3873.870 & 25806.66 & & $275_{9 / 2}-53329 / 2$ & +.02 & -- \\
\hline $2 \mathrm{~h}$ & 3771.252 & 26508.86 & & $244_{5 / 2}-5093_{5 / 2}$ & +.08 & -. & 100 & 3875.26 & 25797.4 & $(.436, .723)$ & $115_{5 / 2}-3738_{5 / 2}$ & -.1 & -- \\
\hline $\begin{array}{l}2 \\
3\end{array}$ & $\begin{array}{l}3774.83 \\
3775.460\end{array}$ & $\begin{array}{l}26483.8 \\
26479.31\end{array}$ & $\ldots$ & $\begin{array}{l}157_{5 / 2}-4225_{3 / 2} \\
194_{5 / 2}-4593_{7 / 2}\end{array}$ & $\begin{array}{r}.0 \\
+.02\end{array}$ & $\cdots$ & & & & $\begin{array}{l}.852,1.134, \\
1.413 .\end{array}$ & & & \\
\hline 30 & 3777.69 & 26463.7 & (.284) 1.241 & $172_{1 / 2}-4370_{3 / 2}$ & $\begin{array}{l}+.02 \\
-.2\end{array}$ & $\cdots$ & 150 & 3876.861 & 25786.75 & $(.171, .512)$ & $157_{5 / 2}-4155_{3 / 2}$ & +.09 & -- \\
\hline 2 & 3778.535 & 26457.77 & & & -.36 & -. & & & & $\begin{array}{l}.797,1.139, \\
1.480 .\end{array}$ & & & \\
\hline & & & & $\begin{array}{r}273^{\prime \prime}{ }_{1 / 2}-588443 / 2 \\
239_{11 / 2}-5040_{9 / 2}\end{array}$ & $\begin{array}{r}+.44 \\
.00\end{array}$ & -- & 20 & 3878.868 & 25773.41 & & $313_{9 / 2}-5 \gamma_{1} \gamma_{7 / 2}$ & +.01 & -- \\
\hline 15 & 3780.357 & 26445.01 & & $3069 / 2-5709_{7 / 2}$ & +.05 & - & 10 & 3879.016 & 25772.42 & & $\begin{array}{l}263_{13 / 2}-5212_{13 / 2} \\
221_{9 / 2}-4793_{7 / 2}\end{array}$ & $\begin{array}{l}+.02 \\
-.09\end{array}$ & $-\cdot$ \\
\hline 2 & 3780.54 & 26443.8 & & $244_{5 / 2}-5086_{7 / 2}$ & +.2 & -. & 2 & 3880.336 & 25763.66 & & $150_{7 / 2}-4082_{5 / 2}$ & $\begin{array}{r}-.09 \\
+.02\end{array}$ & -- \\
\hline 4 & 3781.864 & 26434.48 & & $2759 / 2-539411 / 2$ & +.06 & .- & 40 & 3881.89 & 25753.3 & & $1895_{5 / 2}-4470_{5 / 2}$ & .0 & -- \\
\hline 15 & & & & $194_{5 / 2}-458 \gamma_{3 / 2}$ & -.15 & -- & 5 & 3883.958 & 25739.63 & & $247_{7 / 2}-5046_{5 / 2}$ & +.05 & -- \\
\hline 15 & 3784.179 & 26418.30 & $\left\{\begin{array}{l}(.254, .423) \\
1.256 .\end{array}\right.$ & & .00 & -. & $\begin{array}{r}2 \mathrm{~h} \\
40\end{array}$ & $\begin{array}{l}3884.888 \\
3887.488\end{array}$ & $\begin{array}{l}25733.47 \\
25716.26\end{array}$ & & $244_{5 / 2}-5015_{5 / 2}$ & $\begin{array}{r}.00 \\
-.05\end{array}$ & -- \\
\hline (6) & 3785.40 & 26409.8 & & $221_{9 / 2}-4856_{11 / 2}$ & +.2 & 13 & 40 & 3887.950 & 25713.20 & $(.095,285$, & $173_{7 / 2}-43045 / 2$ & .00 & -- \\
\hline $\begin{array}{r}2 \\
10\end{array}$ & $\begin{array}{l}3785.535 \\
3787.192\end{array}$ & $\begin{array}{l}26408.84 \\
26397.28\end{array}$ & & $217_{7 / 2}-48187 / 2$ & +.04 & -. & & & & .476 & & & \\
\hline $\begin{array}{r}10 \\
150\end{array}$ & $\begin{array}{l}3787.192 \\
3787.520\end{array}$ & $\begin{array}{l}26397.28 \\
26395.00\end{array}$ & $(.102, .308$ & $266_{5 / 2}-5305_{5 / 2}$ & -.03 & -- & $\begin{array}{r}2 \\
40\end{array}$ & $\begin{array}{l}3889.36 \\
3889.962\end{array}$ & $\begin{array}{l}25703.9 \\
25699.91\end{array}$ & & $288_{7 / 2}-5451_{7 / 2}$ & $\begin{array}{l}-.1 \\
+.02\end{array}$ & -- \\
\hline & & & $.514, .720)$ & $150_{7 / 2}-4145_{9 / 2}$ & & -- & $\begin{array}{r}40 \\
1\end{array}$ & $\begin{array}{l}3889.962 \\
3890.54\end{array}$ & $\begin{array}{l}25699.91 \\
25696.1\end{array}$ & (000) 1.123 & $\begin{array}{l}221_{9 / 2}-478 b_{11 / 2} \\
272_{9 / 2}-52857 / 2\end{array}$ & $\begin{array}{l}+.02 \\
.0\end{array}$ & $\begin{array}{l}-- \\
--\end{array}$ \\
\hline & & & $638, .844$ & & & & 5 & 3893.48 & 25676.7 & & $2477 / 2-50409 / 2$ & -.1 & - \\
\hline & & & $\begin{array}{l}1.050,1.254, \\
1.459,1.666\end{array}$ & & & & 5 & 3893.914 & 25673.82 & & $\begin{array}{r}319_{7 / 2}-57659 / 2 \\
164_{3 / 2}-4199_{1 / 2}\end{array}$ & $\begin{array}{l}-.04 \\
-.1\end{array}$ & -- \\
\hline 4 & & & $1.872,2.084$ & & & & 4 & 3895.41 & 25664.0 & & $\left\{194_{5 / 2}-4512_{3 / 2}\right.$ & +.1 & - \\
\hline 4 & $\begin{array}{l}3789.212 \\
3789.771\end{array}$ & $\begin{array}{l}26383.21 \\
26379.32\end{array}$ & - non & $197_{1 / 2}-46141 / 2$ & $\begin{array}{l}+.01 \\
+.03\end{array}$ & -- & 30 & 3896.110 & 25659.35 & (.366) 1.080, & $204_{3 / 2}-46141 / 2$ & -.03 & - \\
\hline 3 & 3795.505 & 26339.47 & & $\begin{array}{l}280_{5 / 2}-54455 / 2 \\
304_{3 / 2}-5686_{3 / 2}\end{array}$ & $\begin{array}{r}+.03 \\
+.01\end{array}$ & -- & 2 & 3898.41 & 256442 & 1.811 & $151_{1 / 2}-4081_{1 / 2}$ & -1 & \\
\hline 10 & 3795.800 & 26337.42 & (0w) $\mathrm{D}$ & $270_{13 / 2}-5346_{11 / 2}$ & -.01 & -. & 2 & $\begin{array}{l}3900.31 \\
3900.34\end{array}$ & $\begin{array}{l}20044.2 \\
25631.5\end{array}$ & $\cdots$ & $231_{5 / 2}-48787 / 2$ & .0 & - \\
\hline 50 & 3796.590 & 26331.94 & $(.108, .319)$ & $115_{5 / 2}-3791_{3 / 2}$ & +.03 & -. & 3 & 3900.50 & 25630.4 & & $204_{3 / 2}-4611_{5 / 2}$ & -.1 & -- \\
\hline 50 & 3797.586 & 26325.04 & $\begin{array}{l}(.192, .574 \\
955) 2.103\end{array}$ & $146_{5 / 2}-40947 / 2$ & -.03 & -- & 30 & 3900.907 & 25627.80 & $\begin{array}{l}(.534, .883, \\
1.235\end{array}$ & $142_{7 / 2}-39847 / 2$ & -.09 & - \\
\hline 3 & 3798.252 & 26320.42 & - & $301_{1 / 2}-5645_{1 / 2}$ & +.11 & -- & 20 & 3901.092 & 25626.58 & & $239_{11 / 2}-4958_{9 / 2}$ & -.03 & -- \\
\hline 3 & 3801.742 & 26296. 26 & & $319_{7 / 2}-582 \gamma_{9 / 2}$ & -.06 & -- & 5 & 3902.580 & 25616.81 & & $2665 / 2-5227_{3 / 2}$ & -.04 & -- \\
\hline & 3802,21 & 26293.0 & & $304_{3 / 2}-5682_{5 / 2}$ & +.11 & -- & $\begin{array}{r}3 \\
10\end{array}$ & 3902.82 & 25615. 2 & (000) 1 190B & $324_{5 / 2}-5805_{3 / 2}$ & -.2 & -- \\
\hline $\begin{array}{l}2 \\
2\end{array}$ & 3804.527 & 26277.01 & & $\begin{array}{l}266_{5 / 2}-5296_{3 / 2} \\
319_{7 / 2}-5826_{7 / 2}\end{array}$ & $\begin{aligned} .0 \\
-03\end{aligned}$ & -- & $\begin{array}{l}10 \\
(5)\end{array}$ & $\begin{array}{l}3905.12 \\
3905.56\end{array}$ & $\begin{array}{l}25600.1 \\
25597.3\end{array}$ & (000) $1.190 \mathrm{~B}_{-}$ & $\begin{array}{l}271_{7 / 2}-527 / 49 / 2 \\
263_{13 / 2}-519411 / 2\end{array}$ & $\begin{array}{l}\overline{7} .1 \\
+.3\end{array}$ & $\overline{13}$ \\
\hline 5 & 3805.41 & 26270.9 & & $\begin{array}{l}319_{7 / 2}-5826_{7 / 2} \\
285_{7 / 2}-5481_{9 / 2}\end{array}$ & $\begin{array}{l}-.00 \\
-.1\end{array}$ & - & 3 & $\begin{array}{l}3905.56 \\
3907.20\end{array}$ & 25586.5 & & $247_{7 / 2}-5031_{7 / 2}$ & .0 & -- \\
\hline 40 & 3807.742 & 26254.82 & $(.064, .192$, & $150_{7 / 2}-4131_{5 / 2}$ & -.01 & -- & 3 & 3907.74 & 25583.0 & & $217_{7 / 2}-47357 / 2$ & .0 & -- \\
\hline & & & $\begin{array}{l}.318) .830 \\
.957,1.084\end{array}$ & & & & 40 & 3908.210 & 25579.91 & $\begin{array}{l}(.155, .257, \\
.360, .453)\end{array}$ & $272_{9 / 2}-527 / 49 / 2$ & -.04 & -- \\
\hline 10d & 3808.200 & 26251.67 & - - - n-n & $204_{3 / 2}-4673_{3 / 2}$ & +.02 & -. & & & & 1.152. & & & \\
\hline 2 & 3808.640 & 26248.64 & & $271_{7 / 2}-5338_{5 / 2}$ & +.04 & -- & 1 & 3909.84 & 25569. 23 & & $273^{\prime \prime}{ }_{1 / 2}-5296_{3 / 2}$ & -.03 & -- \\
\hline 3 & 3809.752 & 26240.98 & $\ldots$ & $239_{11 / 2}-5019_{9 / 2}$ & +.04 & -- & 2 & 3910.157 & 25567.18 & & $273_{1 / 2}^{\prime}-5295_{3 / 2}$ & -.01 & -- \\
\hline 20 & & 26238.59 & & $1737 / 2-4356_{5 / 2}$ & +.05 & .. & 2 & 3910.272 & & & $273^{\prime \prime}{ }_{1 / 2}-5295_{3 / 2}$ & +.03 & -- \\
\hline 20 & 3812.259 & 26223.72 & (.160) $1.192 \mathrm{~A}$ & $273_{11 / 2}-5346_{11 / 2}$ & +.03 & -- & 2 & 3911. 76 & 25556.7 & & $266_{5 / 2}-5221_{5 / 2}$ & & -- \\
\hline & 3812.812 & 26219.92 & & $266_{5 / 2}-5288_{5 / 2}$ & +.01 & & 60 & 3913.924 & 25542.57 & (.089) $1.291 \mathrm{~A}_{-}$ & $157_{5 / 2}-4181_{5 / 2}$ & -.03 & -- \\
\hline$(1 \mathrm{H})$ & 3814.24 & 26210.1 & & $247_{7 / 2}-5093_{5 / 2}$ & +.1 & 7 & 200 & 3917.270 & 25520.75 & $(.141 \mathrm{w}) 1.142 \mathrm{C}$ & $1669 / 2-421 / 49 / 2$ & -.03 & -- \\
\hline 40 & 3815.655 & 26200.38 & $(0 \mathrm{~W}) 1.107 \mathrm{C}_{\ldots} \ldots$ & $146_{5 / 2}-4082_{5 / 2}$ & +.01 & 19 & 10 & 3919.076 & 25508. 99 & & $221_{9 / 2}-47667 / 2$ & +.02 & -- \\
\hline 2 & 3816.294 & 26195. 99 & & $266_{5 / 2}-52857 / 2$ & -.02 & -- & 20 & 3920.85 & 25497.5 & $(0$ W) $1.189 \mathrm{~B}$ & $273_{11 / 2}-527 / 49 / 2$ & +.1 & -- \\
\hline 30 & 3817.539 & 26187.45 & $(.183, .549)$ & $146_{5 / 2}-4080_{3 / 2}$ & +.06 & -. & 10 & 3922. 274 & 25488.19 & $(.226, .682)$ & $194_{5 / 2}-44947 / 2$ & -.04 & -- \\
\hline 5 & 3818.655 & 26179. 79 & & $271_{7 / 2}-5332_{9 / 2}$ & -.03 & & & & & $.226, .682$ & & & \\
\hline (8) & 3820.45 & 26167.5 & & $204_{3 / 2}-46645 / 2$ & -.2 & 13 & 5 & 3923.58 & 25479.7 & & $115_{5 / 2}-3706_{3 / 2}$ & & -- \\
\hline 3 & 3821.598 & 26159.64 & & $272_{9 / 2}-53322_{9 / 2}$ & +.04 & -. & 10 & & & $(.224) \ldots \ldots$ & $224_{3 / 2}-4789_{\mathrm{s} / 2}$ & +.01 & -- \\
\hline $2 \mathrm{~h}$ & 3821.962 & 26157.15 & & $217_{7 / 2}-47937 / 2$ & .00 & -- & 4 & 3924.67 & 25472.6 & & $247_{7 / 2}-5019_{8 / 2}$ & -.1 & -- \\
\hline 3 & 3823.478 & 26146.77 & & $280_{5 / 2}-5417_{7 / 2}$ & -.16 & -- & 2 & 3925.038 & 25470.24 & & $244_{5 / 2}-4989_{3 / 2}$ & +.07 & -- \\
\hline 10 & 3823.772 & 26144.76 & -- & $\begin{array}{l}261_{3 / 2}-522 \gamma_{3 / 2} \\
247_{7 / 2}-50867 / 2\end{array}$ & $\begin{array}{l}+.06 \\
-.02\end{array}$ & -- & $\begin{array}{r}2 \\
10\end{array}$ & $\begin{array}{l}3925.63 \\
3926.522\end{array}$ & $\begin{array}{l}25466.4 \\
25460.62\end{array}$ & $(.238)$ & $\begin{array}{l}278_{3 / 2}-5329_{3 / 2} \\
278_{3 / 2}-5328_{1 / 2}\end{array}$ & $\begin{array}{l}+.3 \\
-.01\end{array}$ & - \\
\hline 15 & 3827.04 & 26122.4 & & $\begin{array}{l}24 i 7 / 2-50867 / 2 \\
1737 / 2-43459 / 2\end{array}$ & -1 & -- & 4 & 3926,85 & 25458.5 & $(.18$ & $261_{3 / 2}-5159 \mathrm{~s} / 2$ & .0 & - \\
\hline 9 & 3827.625 & 26118.45 & $(.201)$ & $197_{1 / 2}-458 \gamma_{3 / 2}$ & +.02 & 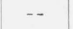 & 4 & 3926.85 & $20+50.0$ & $\left(\begin{array}{lll}227 & 603\end{array}\right)$ & $288_{7 / 2}-542 \delta_{7 / 2}$ & +.2 & -- \\
\hline 50 & 3828.325 & 26113.67 & (.149) $1.245 \mathrm{~A}_{-}$ & $115_{5 / 2}-3769_{5 / 2}$ & -.03 & -- & 30 & 3927.59 & 25453.7 & $(.227, .693) \ldots$ & $142_{7 / 2}-3967_{5 / 2}$ & .0 & -- \\
\hline
\end{tabular}


Table 2. Classified lines of Re $\mathrm{I}$-Continued

\begin{tabular}{|c|c|c|c|c|c|c|c|c|c|c|c|c|c|}
\hline Int. & $\lambda_{\mathrm{vac}}$ & $\sigma_{\mathrm{vac}}$ & Zeeman effect & Transition & $\mathrm{O}-\mathrm{C}$ & Notes & Int. & $\lambda_{\mathrm{vac}}$ & $\sigma_{\mathrm{vac}}$ & Zeeman effect & Transition & $\mathrm{O}-\mathrm{C}$ & Notes \\
\hline 2 & $\begin{array}{c}A \\
3928.364\end{array}$ & $\underset{25448.68}{K}$ & & $2720 / 2-52617 / 2$ & +02 & & 10 & $\begin{array}{c}A \\
4040.178\end{array}$ & $\begin{array}{c}K \\
24744.39\end{array}$ & (080) 1 211 & $231=12-4889=$ & -02 & \\
\hline 15 & 3928. 704 & 25446.48 & $(.130, .395)$ & $\begin{array}{l}2729 / 2-52017 / 2 \\
261_{3 / 2}-5157_{3 / 2}\end{array}$ & .00 & $\begin{array}{l}-- \\
--\end{array}$ & 3 & 4041.305 & 24737.49 & . & $\begin{array}{l}2015 / 2-4 / 87_{5 / 2} \\
324_{5 / 2}-5 \gamma_{1 / 7 / 2}\end{array}$ & $\begin{array}{l}-.02 \\
-.07\end{array}$ & -- \\
\hline 6 & 3929.198 & 25443.27 & & $194_{5 / 2}-4490_{7 / 2}$ & +.01 & -- & 3 & 4044.798 & 24716.12 & & $311_{5 / 2}-5590_{3 / 2}$ & +.19 & 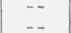 \\
\hline 200 & 3929.847 & 25439.07 & $(.067, .202$ & $142 \pi / 2-39659 / 2$ & -.06 & - & 4 & 4045.237 & 24713.44 & & $298_{5 / 2}-54517 / 2$ & -.02 &.- \\
\hline & & & $.338, .474)$ & & & & 2 & $\begin{array}{l}4047.106 \\
4048.99\end{array}$ & $\begin{array}{l}24702.03 \\
24690.6\end{array}$ & $(1$ & $273_{11 / 2}-519411 / 2$ & -.03 & -. \\
\hline & & & $1.239,1.374$. & & & & & & & $1.036,1.249$, & $-44143 / 2$ & .0 & -- \\
\hline $\begin{array}{r}6 \\
20\end{array}$ & $\begin{array}{l}3930.53 \\
3931.201\end{array}$ & $\begin{array}{l}25434.7 \\
25430.31\end{array}$ & $(0 \mathrm{w}) .972 \mathrm{C}$ & $\begin{array}{l}2477 / 2-5015_{5 / 2} \\
2177 / 2-47209 / 2\end{array}$ & $\begin{array}{l}.0 \\
-.02\end{array}$ & -- & 10 & 4050.926 & 24678.74 & $\begin{array}{l}1.462,1.676 \\
(.058, .174)\end{array}$ & $224_{3,2}-471 G_{5 / 2}$ & -.04 & -- \\
\hline 2 & 3936. 70 & 25394.8 & & $2043 / 2-4587_{3 / 2}$ & +.2 & -- & & & & 1.073 . & & & \\
\hline 100 & $\begin{array}{l}3936.899 \\
3927\end{array}$ & $\begin{array}{l}25393.51 \\
25388.81\end{array}$ & (000) 1.321 & & +.02 & -. & 3 & 4052.852 & 24667.01 & & $298_{5 / 2}-5446_{3 / 2}$ & -.01 & -- \\
\hline $2 \mathrm{~h}$ & 3937.628 & $\begin{array}{l}25388.81 \\
25364.07\end{array}$ & $(156) 520$ & $319_{7 / 2}-5737_{7 / 2}$ & +.05 & -- & $\begin{array}{r}3 \\
15\end{array}$ & $\begin{array}{l}4053.36 \\
4054.265\end{array}$ & $\begin{array}{l}24663.9 \\
24658.41\end{array}$ & & $305_{11 / 2}-5522_{11 / 2}$ & $\begin{array}{r}.0 \\
-01\end{array}$ & - \\
\hline 6 & 3941.468 & 25364.07 & $\begin{array}{l}(.156) .520 \\
.828\end{array}$ & $197_{1 / 2}-4512_{3 / 2}$ & +.17 & -- & $20 \mathrm{H}$ & $\begin{array}{l}4054.265 \\
4055.330\end{array}$ & $\begin{array}{l}24658.41 \\
24651.93\end{array}$ & & $\begin{array}{r}1971 / 2-444 I_{1 / 2} \\
3340_{5 / 2}-58067 / 2\end{array}$ & $\begin{array}{l}\overline{-} .01 \\
+.02\end{array}$ & -- \\
\hline (2) & 3942.22 & 25359.2 & & $280_{5 / 2}-53388_{5 / 2}$ & -.2 & 13 & 10 & 4057. C01 & 24641.78 & (.213) .668. & $278_{3 / 2}-5246_{1 / 2}$ & -.02 & -- \\
\hline 10 & 3942.562 & 25357.03 & & $224_{3 / 2}-4777_{3 / 2}$ & -.05 & - & 2 & 4059.753 & 24625.08 & & $231_{5 / 2}-477 \gamma_{3 / 2}$ & -.02 & -. \\
\hline 15 & 3944.344 & 25345.58 & & $261_{3 / 2}-5147_{5 / 2}$ & .00 & -- & 3 & 4061.643 & 24613.62 & & $239_{11 / 2}-4856_{11 / 2}$ & -.01 & -- \\
\hline 50 & 3944.718 & 25343.17 & $(.075, .226)$ & $275_{9 / 2}-5285_{7 / 2}$ & +.04 & - & 30 & 4061. 856 & $\begin{array}{l}24612.33 \\
24609.0\end{array}$ & (0w) .1.241B & $150_{7 / 2}-3967_{5 / 2}$ & $\begin{array}{r}.00 \\
-.2\end{array}$ & $-\overline{7}$ \\
\hline 150 & 3945.906 & 25335.55 & $\begin{array}{l}(.374) 1.068, \\
1.815 \text {. }\end{array}$ & $204_{3 / 2}-4581_{1 / 2}$ & +.02 & -- & $\begin{array}{l}(4 \mathrm{~h}) \\
15 \mathrm{~h}\end{array}$ & $\begin{array}{l}4062.40 \\
4064.328\end{array}$ & $\begin{array}{l}24609.0 \\
24597.36\end{array}$ & & $\begin{array}{l}298_{5 / 2}-54400_{5 / 2} \\
244_{5 / 2}-4902_{5 / 2}\end{array}$ & $\begin{array}{l}-.2 \\
-.01\end{array}$ & $\begin{array}{c}7 \\
--\end{array}$ \\
\hline 2 & 3946.75 & 25330.1 & & $239_{11 / 2}-4928_{11 / 2}$ & .0 & -- & 2 & 4066.94 & 24581.6 & & $224_{3 / 2}-4700_{3 / 2}$ & +.1 & - \\
\hline 15 & 3950.570 & 25305.64 & $\begin{array}{l}(.204, .611) \\
1.377 .\end{array}$ & $2805 / 2-5333_{7 / 2}$ & +.02 & -- & 2 & 4067.246 & 24579.71 & & $\begin{array}{l}288_{7 / 2}-5338_{5 / 2} \\
280_{5 / 2}-5261_{7 / 2}\end{array}$ & $\begin{array}{l}-.15 \\
+.02\end{array}$ & -- \\
\hline 10 & 3950.64 & 25305.2 & & $146_{5 / 2}-3992_{3 / 2}$ & -.1 & -. & 1 & 4067.44 & 24578.5 & & $3069 / 2-5522_{11 / 2}$ & .0 & -- \\
\hline 2 & 3951.45 & 25300.0 & & $313_{9 / 2}-5669_{7 / 2}$ & -.1 & -. & 10 & 4069.16 & 24568.2 & & $266_{5 / 2}-5122_{7 / 2}$ & .0 & - \\
\hline 2 & 3951.836 & 25297.53 & & $278_{3 / 2}-5312_{3 / 2}$ & -.01 & -- & 1 & 4072. 36 & 24548.9 & & $231_{5 / 2}-4770_{3 / 2}$ & -.1 & -- \\
\hline 15 & 3954.428 & 25280.95 & (1.017) .438, & $224_{3 / 2}-477 C_{3 / 2}$ & .00 & - & 4 & 4073. 060 & $\begin{array}{l}24544.63 \\
24514.17\end{array}$ & $(000) 1.231$ & $\begin{array}{l}166_{9 / 2}-4116_{7 / 2} \\
231_{5 / 2}-4766_{7 / 2}\end{array}$ & $\begin{array}{r}.00 \\
-.03\end{array}$ & -- \\
\hline 2 & 3956.60 & 25267.1 & & $306_{9 / 2}-5591_{9 / 2}$ & .0 & -- & 10 & 4079.364 & 24506. 70 & $(.160)$ & $271_{7 / 2}-51647 / 2$ & +.01 & -- \\
\hline 3 & 3957.173 & 25263.41 & & $280_{5 / 2}-5329_{3 / 2}$ & +.01 & -. & 80 & 4081. 432 & 24494. 29 & $(.291, .874)$ & $164_{3 / 2}-4082_{5 / 2}$ & -.03 & - \\
\hline 10 & 3957.363 & 25262.20 & $(000) 1.385$ & $194_{5 / 2}-4472_{3 / 2}$ & .00 & -- & & & & $.259, .831$ & & & \\
\hline 7 & 3958.357 & 25255.86 & & $306_{9 / 2}-55897 / 2$ & $\begin{array}{l}-.01 \\
-.07\end{array}$ & -- & & & 24487.0 & 1.413. & $-52009 / 2$ & -.1 & \\
\hline 15 & 3960.552 & 25241.86 & $\{(.095) \ldots$ & $\begin{array}{l}2243 / 2-4001_{1 / 2} \\
244_{5 / 2}-4966_{3 / 2}\end{array}$ & $\begin{array}{l}\overline{-} .01 \\
+.01\end{array}$ & -- & 20 & 4083.365 & 24482.69 & (.271) 1.970 & $164_{3 / 2}-4981_{1 / 2}$ & +.03 & 17 \\
\hline 150 & 3961.036 & 25238.77 & $(.073, .220)$ & $138_{3 / 2}-39066_{5 / 2}$ & -.03 & - & 20 & 4083.584 & 24481. 38 & & $164_{3 / 2}-4080_{3 / 2}$ & +.04 & 17 \\
\hline & & & $\begin{array}{l}1.120,1.267 \\
1.414 \\
1.562\end{array}$ & & & & 4 & 4087.89 & $\begin{array}{l}24455.6 \\
24454.4\end{array}$ & & $3387 / 2-58279 / 12$ & $\begin{array}{l}-.1 \\
-.4\end{array}$ & -- \\
\hline 300 & 3962.484 & 25229.55 & $(0 \mathrm{~W}) 1.781 \mathrm{~W}$ & $164_{3 / 2}-4155_{3 / 2}$ & -.02 & $\ldots$ & $\begin{array}{l}4 \\
1\end{array}$ & 4088.69 & $\begin{array}{l}24404.4 \\
24450.8\end{array}$ & & $278_{3 / 2}-5227_{3 / 2}$ & $\begin{array}{l}\overline{7} .4 \\
+.2\end{array}$ & $-\overline{5}$ \\
\hline 40 & 3963.267 & 25224.57 & (0w) $1.099 \mathrm{~h}$ & $1669 / 2-41847 / 2$ & .00 & -- & 5 & 4089. 01 & 24448.9 & & $271_{7 / 2}-5159_{5 / 2}$ & -1 & $\begin{array}{r}5 \\
--\end{array}$ \\
\hline 7 & 3963.707 & 25221.77 & - & $32441 / 2-5766_{3 / 2}$ & -.05 & $\ldots$ & 4 & 4089.407 & 24446.52 & & $247_{7 / 2}-4917_{9 / 2}$ & -.02 & -- \\
\hline 5 & 3964. 279 & 25218.13 & -..... & $341_{9 / 2}-5941_{11 / 2}$ & -.06 & -- & 30 & 4089.919 & 24443.46 & (.442) 1.247, & $146_{5 / 2}-3906_{5 / 2}$ & .00 & - \\
\hline 2 & 3964.53 & $\begin{array}{l}25216.5 \\
25214.75\end{array}$ & (070 209 & $305_{11 / 2}-557 \gamma_{13 / 2}$ & -.1 & -- & & & & 1.435 & & & \\
\hline 15 & 3964.810 & 25214.75 & $\begin{array}{l}(.070, .209 \\
.348, .487\end{array}$ & $239_{11 / 2}-4917_{9 / 2}$ & & -- & $\begin{array}{l}2 \\
4\end{array}$ & $\begin{array}{l}4090.59 \\
4091.12\end{array}$ & $\begin{array}{l}24439.4 \\
24436.3\end{array}$ & & $\begin{array}{l}261_{3 / 2}-5057_{3 / 2} \\
338_{7 / 2}-58267 / 2\end{array}$ & $\begin{array}{r}-.1 \\
.0\end{array}$ & -- \\
\hline & & & $.626) .365$ & & & & $(5 \mathrm{~h})$ & 4093.02 & 24424.9 & & $341_{9 / 2}-5861_{11 / 2}$ & -.2 & $\overline{13}$ \\
\hline 1 & 3965.27 & 25211.8 & & $272_{9 / 2}-5237_{7 / 2}$ & .0 & -- & $2 \mathrm{~h}$ & & & & $324_{5 / 2}-5682_{5 / 2}$ & -.2 & $\ldots$ \\
\hline 30 & 3967.39 & 2519 & (0w) $.140 \mathrm{w}_{-}$ & $221_{9 / 2}-47355_{7 / 2}$ & +.1 & -. & $3 \mathrm{~h}$ & 4103.763 & 24361.00 & & $244_{5 / 2}-48787 / 2$ & +.05 & -- \\
\hline 2 & 3967.707 & 25196.34 & - & $285_{7 / 2}-53737 / 2$ & +.01 & $-\overline{7}$ & 70 & 4104. 422 & 24357.09 & (000) $1.330_{\ldots}$ & $194_{5 / 2}-43817 / 2$ & -.03 & -- \\
\hline (1h) & 3970.93 & 25175.9 & & $157_{5 / 2}-40947 / 2$ & -.2 & 7 & 10 & 4106.440 & 24345.12 & (000) $1.188 \mathrm{~h}$ & $271_{7 / 2}-51489 / 2$ & -.04 & -- \\
\hline 3 & 3975. 374 & 25147.75 & $\ldots$ & $244_{5 / 2}-4957_{5 / 2}$ & +.03 & -. & $5 \mathrm{~h}$ & 4106.829 & 24342.81 & & $280_{5 / 2}-5237_{7 / 2}$ & 十. 01 & -- \\
\hline 20 & 3975.648 & 25146.01 & & $163_{11 / 2}-4145_{9 / 2}$ & -.02 & -- & $2 \mathrm{~h}$ & 4107.431 & 24339.25 & & $333_{11 / 2}-57659 / 2$ & -.03 & -- \\
\hline 4 & 3976.759 & 25138.99 & $-\ldots$ & $247_{7 / 2}-49867 / 2$ & -.01 & -- & $2 \pi$ & 807 & 2433687 & & $285_{7 / 2}-5288_{5 / 3}$ & $\begin{array}{l}+.04 \\
+03\end{array}$ & -- \\
\hline 7 & 3982. 286 & 25104.10 & & $1895_{5 / 2}-4405_{5 / 2}$ & .0 & -- & 1 & 4107. 832 & $\begin{array}{l}24336.87 \\
24336.03\end{array}$ & & & $\begin{array}{r}+.03 \\
+01\end{array}$ & -- \\
\hline 10 & $\begin{array}{l}3983.41 \\
3985.31\end{array}$ & $\begin{array}{l}25097.0 \\
25085.0\end{array}$ & $\cdots$ & $270_{13 / 2}-52222_{13 / 2}$ & -.1 & -- & $\begin{array}{r}3 \\
10\end{array}$ & 4107. 973 & $\begin{array}{l}24336.03 \\
24332.22\end{array}$ & $(.241, .744)$ & $\begin{array}{l}271_{7 / 2}-51475 / 2 \\
261_{3 / 2}-5046_{5 / 2}\end{array}$ & $\begin{array}{l}+.01 \\
-.01\end{array}$ & -- \\
\hline 1 & 3985.42 & 25084.3 & & $\begin{array}{r}273^{\prime}{ }_{1 / 2}-5246_{1 / 2} \\
273^{\prime \prime}{ }_{1 / 2}-5246_{1 / 2}\end{array}$ & $\begin{array}{r}-.1 \\
.0\end{array}$ & -- & 10 & 4108.618 & & $1.408,1.884$ & & & \\
\hline $5 \mathrm{~h}$ & 3987.086 & 25073.88 & & $3259_{3 / 2}-5766_{3 / 2}$ & +.08 & -- & 1 & 4109. 451 & 24327. 28 & & $166_{9 / 2}-40947 / 2$ & +.03 & $-\cdot$ \\
\hline 15 & 3990.660 & 25051.42 & (n) & $\left(\begin{array}{l}261_{3 / 2}-51183 / 2 \\
1575 / 2-4082\end{array}\right.$ & -.01 & -- & $1 \mathrm{CO}$ & 4110.894 & 24318.74 & (.435) 1.219, & $172_{1 / 2}-4155_{3 / 2}$ & -.04 & -- \\
\hline 30 & 3991.033 & 25049.08 & (.130) 1.012, & $\begin{array}{l}157_{5 / 2}-4088_{5 / 2} \\
146_{5 / 2}-3967_{5 / 2}\end{array}$ & $\begin{array}{l}+.01 \\
+.02\end{array}$ & 11 & 5 & 4111.598 & 24314. 58 & 2.091 & $263_{13 / 2}-5066_{11 / 2}$ & +.03 & -- \\
\hline & 3991.571 & 25045,70 & 1.21 & & & - & & 4112. 275 & 24310.58 & & $224_{3 / 2}-4673_{3 / 2}$ & $\begin{array}{r}+.03 \\
+\quad 03\end{array}$ & -- \\
\hline 10 & 3992.72 & 25038.5 & (n) & $\begin{array}{l}221_{9 / 2}-47200_{9 / 2} \\
157_{5 / 2}-4080_{3 / 2}\end{array}$ & $\begin{array}{l}+.01 \\
+.1\end{array}$ & $\begin{array}{l}-- \\
--\end{array}$ & 30 & 4113.402 & & $\begin{array}{l}(.184, .545 \\
.905,1.266)\end{array}$ & $1427 / 2-38527 / 2$ & & - \\
\hline $4 d$ & 3994. 154 & 25029.50 & & $\begin{array}{l}10 / 51^{5}-40003 / 2 \\
231_{5 / 2}-48187 / 2\end{array}$ & +.11 & - & & & & .684, 1.266) & & & \\
\hline 3 & 3996. 987 & 2501 & & $313_{9 / 2}-5641_{9 / 2}$ & -.15 & -- & & & & $1.383,1.727$. & & & \\
\hline 3 & 4000.122 & 24992.17 & -..- & $270_{13 / 2}-5212_{13 / 2}$ & -.02 & -. & 3 & 4114. 676 & 24296.39 & $(.129, .389)$ & $304_{3 / 2}-5482_{5 / 2}$ & -.03 & -- \\
\hline 3 & 4001.19 & 24985.5 & - & $164_{3 / 2}-4131_{5 / 2}$ & .0 & -- & 10 & 4121. 548 & 24255.88 & $(0 \mathrm{w}) 2.010 \mathrm{~B}$ & $20447 / 2-44705 / 2$ & $\begin{array}{l}+.3 \\
-.2\end{array}$ & 27 \\
\hline 70 & 4004.930 & 24962.16 & $\begin{array}{l}\text { (.174) 1.157, } \\
\mathbf{1 . 5 0 3}\end{array}$ & $197_{1 / 2}-4472_{3 / 2}$ & -.02 & -- & $\begin{array}{r}30 \\
8\end{array}$ & $\begin{array}{l}4121.635 \\
4122.738\end{array}$ & $\begin{array}{l}24255.37 \\
24248.88\end{array}$ & $(.132, .399) \ldots$ & $288_{7 / 2}-5305_{5 / 2}$ & $\begin{array}{l}\overline{7} .2 \\
+.01\end{array}$ & .. \\
\hline 3 & 4010,322 & 24928.60 & & $266_{5 / 2}-5159_{5 / 2}$ & -.05 & -- & 2 & 4122.896 & 24247.95 & & $280_{5 / 2}-5227_{3 / 2}$ & -.01 & -. \\
\hline & 4010.022 & 24921.19 & & $288_{7 / 2}-5373_{7 / 2}$ & +.01 & & 2 & 4123.507 & 24244,36 & & $194_{5 / 2}-4370_{3 / 2}$ & +.05 & -- \\
\hline $\begin{array}{r}(35) \\
15\end{array}$ & $\begin{array}{l}4011.514 \\
4012.26\end{array}$ & $\begin{array}{l}24921.19 \\
24916.6\end{array}$ & 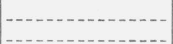 & $280_{5 / 2}-5295_{3 / 2}$ & -.08 & 3 & 2 & 4123.82 & 24242.5 & & $273_{11 / 2}-5148_{9 / 2}$ & +.1 & -- \\
\hline & $\begin{array}{l}4012.26 \\
4018.40\end{array}$ & 24878.5 & (000) 1.160 & $2665 / 2-5157_{3 / 2}$ & .0 & -- & 2 & $\begin{array}{l}4128.002 \\
4130.458\end{array}$ & $\begin{array}{l}24217.95 \\
24203.56\end{array}$ & (0w) 1.084 & & $\begin{array}{l}+.14 \\
+.01\end{array}$ & -- \\
\hline $\begin{array}{r}20 \\
6\end{array}$ & 4019. 124 & 24874.01 & (000) $1.160 \ldots$ & $273_{11 / 2}-5212_{13 / 2}$ & $\begin{aligned} .0 \\
-.01\end{aligned}$ & - & 8 & 4130.458 & 24203.56 & $\int(0 \mathrm{~W}) 1.041 \mathrm{C}$ & $\begin{array}{l}231_{5 / 2}-47357 / 2 \\
221_{9 / 2}-46357 / 2\end{array}$ & T. 05 & $2 \overline{2}$ \\
\hline 10 & 4022.86 & 24850.9 & & $\begin{array}{l}217_{7 / 2}-4664_{45 / 2} \\
280_{5 / 2}-5288_{5 / 2}\end{array}$ & $\begin{array}{l}-.01 \\
-.1\end{array}$ & -- & 10 & 4132.277 & 24192.90 & 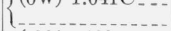 & $273^{\prime \prime}{ }_{1 / 2}-5157_{3 / 2}$ & +.05 & $\ldots$ \\
\hline 60 & & & & $194_{5 / 2}-4430_{5 / 2}$ & +.1 & -- & 100 & 4133.425 & 24186.18 & $(.061, .183$ & $163_{11 / 2}-4049_{13 / 2}$ & -.01 & -- \\
\hline 60 & 4022.965 & 24850.26 & $\begin{array}{l}(.176, .525) \\
.553, .907\end{array}$ & $204_{3 / 2}-4533_{5 / 2}$ & -.04 & -- & & & & $\begin{array}{l}.305, .426 \\
.548, .669)\end{array}$ & & & \\
\hline 40 & 4023.313 & 24848.11 & 1.260. & $142_{7 / 2}-3906_{5 / 2}$ & & & & & & $1.151 \ldots$ & & & \\
\hline $3 \mathrm{~d}$ & 4025.07 & 24837.3 & & $301_{1 / 2}-5496_{1 / 2}$ & .0 & -- & 200 & 4136.45 & 24168.5 & (.464) $1.516 \mathrm{~A}$ & $117_{9 / 2}-3592_{9 / 2}$ & .0 & -. \\
\hline 9 & 4025.614 & 24833,91 & & $2857 / 2-5337_{9 / 2}$ & +.1 & -- & 20 & 4137. 603 & 24161. 76 & $(.235,387$ & $217_{7 / 2}-45937 / 2$ & -.02 & $\cdots$ \\
\hline $\begin{array}{l}9 \\
3\end{array}$ & $\begin{array}{l}4025.614 \\
4026.707\end{array}$ & $\begin{array}{l}24833.91 \\
24827.17\end{array}$ & & $\begin{array}{l}166_{9 / 2}-41459 / 2 \\
280_{5 / 2}-52857 / 2\end{array}$ & $\begin{array}{l}+.01 \\
+.05\end{array}$ & -- & & & & & & & \\
\hline 4 & 4027.124 & 24824.60 & $(.102, .304)$ & $244_{5 / 2}-4925_{3 / 2}$ & -.02 & $\cdots$ & 3 & 4138.532 & & & & & \\
\hline 30 & 4028.53 & 24815.9 & (0w) 1.168 & $231_{5 / 2}-4797_{5 / 2}$ & -.1 & 22 & (1h) & 4142. 44 & $\begin{array}{l}24133.64 \\
24133.6\end{array}$ & & $\begin{array}{l}15 / 5 / 2-59923 / 2 \\
2759 / 2-51647 / 2\end{array}$ & $\begin{array}{l}+.01 \\
+.1\end{array}$ & 23 \\
\hline & & & & $270_{13 / 2}-519411 / 2$ & +.1 & & & 4142. 767 & 24131. 65 & & $261_{3 / 2}-50266_{3 / 2}$ & 00 & - \\
\hline 60 & 4029.63 & 24809.2 & (0w) $1.212 \mathrm{~B}$ & $\begin{array}{l}1737 / 2-42149 / 2 \\
3069 / 2-55457 / 2\end{array}$ & $\begin{array}{l}.0 \\
.0\end{array}$ & -- & 200 & 4144.363 & 24122.35 & $\begin{array}{l}(.061, .183, \\
.306, .428)\end{array}$ & $173_{7 / 2}-4145_{9 / 2}$ & -.01 & -- \\
\hline 8 & 4032.143 & 24793.70 & & $2857 / 2-53337 / 2$ & -.11 & -- & & & & $1.195,1.319$, & & & \\
\hline 100 & 4033.31 & 24786.5 & $(.208 \mathrm{w}) 1.152 \mathrm{~A}$ & $\begin{array}{l}272_{9 / 2}-51957 / 2 \\
150_{7 / 2}-39847 / 2\end{array}$ & $\begin{array}{l}+.03 \\
-.1\end{array}$ & -- & & & & $\begin{array}{l}1.442,1.566 \\
1.689,1.811 .\end{array}$ & & & \\
\hline 10 & 4033.63 & 24784.6 & & $272_{9 / 2}-519411 / 2$ & .0 & -- & 10 & 4146. 233 & 24111.47 & $(.074, .223$ & $194_{5 / 2}-43566_{5 / 2}$ & .00 & -- \\
\hline $1 \mathrm{~h}$ & 4034.74 & 24777.7 & - - - & $\begin{array}{l}1427 / 2-38999 / 2 \\
231_{5 / 2}-47937 / 2\end{array}$ & $\begin{array}{r}.2 \\
.0\end{array}$ & -- & 50 & 4149. 96 & 24089.8 & $(0 \mathrm{w}) 1.484 \mathrm{~A} \ldots$ & $138_{3 / 2}-3791_{3 / 2}$ & .0 & -- \\
\hline 50 & 4037.49 & 24760.9 & (.813). .073 , & $151_{1 / 2}-3992_{3 / 2}$ & .0 & -- & 10 & 4150.527 & 24086.53 & - & $224_{3 / 2}-4650_{5 / 2}$ & $\begin{array}{l}-.04 \\
-.03\end{array}$ & 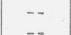 \\
\hline & & & & $34527 / 2-59265 / 2$ & -1 & & $10 \mathrm{~h}$ & 4152.29 & 24076.3 & & $3358_{3 / 2}-5766_{3 / 2}$ & .0 & .. \\
\hline
\end{tabular}


TABle 2. Classified lines of $\mathrm{Re} \mathrm{I}$-Continued

\begin{tabular}{|c|c|c|c|c|c|c|c|c|c|c|c|c|c|}
\hline Int. & $\lambda_{\text {vac }}$ & $\sigma_{\mathrm{vac}}$ & Zeeman effect & Transition & $\mathrm{O}-\mathrm{C}$ & Notes & Int. & $\lambda_{\text {vac }}$ & $\sigma_{\mathrm{vac}}$ & Zeeman effect & Transition & $\mathrm{O}-\mathrm{C}$ & Notes \\
\hline & $\underset{4152.632}{A}$ & $\begin{array}{c}K \\
24074.32\end{array}$ & & & & & & A & $K$ & & & & \\
\hline $\begin{array}{r}30 \\
3\end{array}$ & $\begin{array}{l}4152.632 \\
4157.252\end{array}$ & $\begin{array}{l}24014.32 \\
24047.57\end{array}$ & & $\begin{array}{l}157_{5 / 2}-39847 / 2 \\
288_{7 / 2}-52857 / 2\end{array}$ & $\begin{array}{r}-.01 \\
.00\end{array}$ & -- & 30 & 4291.654 & 23294.48 & & $\begin{array}{l}157_{5 / 2}-3906_{5 / 2} \\
146_{5 / 2}-3 \% 91_{3 / 2}\end{array}$ & $\begin{array}{l}-.02 \\
+.07\end{array}$ & - \\
\hline 20 & 4159.925 & 24032. 11 & $(0 \mathrm{w}) .7$ & $272_{9 / 2}-5119_{9 / 2}$ & -.03 & 19 & 2 & 4294.62 & 23278.4 & & $244_{5 / 2}-4770_{3 / 2}$ & $\begin{array}{c}+.06 \\
.0\end{array}$ & -- \\
\hline 5 & 4160.773 & 24027.22 & (20) & $261_{3 / 2}-5015_{5 / 2}$ & -.08 & -. & 20 & 4299.917 & 23249.72 & & $239_{11 / 2}-4720_{9 / 2}$ & -.01 & -- \\
\hline 1 & 4164.32 & 24006.8 & & $1507 / 2-3906_{5 / 2}$ & +.1 & -- & 4 & 4301.052 & 23243.58 & & $244_{5 / 2}-4766_{7 / 2}$ & -.03 & - \\
\hline 10 & 4166.408 & 23994. 72 & & $305_{11 / 2}-5455_{11 / 2}$ & +.02 & - & 4 & 4301. 774 & 23239.68 & & $2729 / 2-50409 / 2$ & +.02 & -. \\
\hline 15 & 4168.588 & 23982.17 & (0w) 1.22 & $1737 / 2-41315 / 2$ & $\begin{array}{l}-.03 \\
-.201\end{array}$ & -- & $\begin{array}{r}3 \mathrm{~d} \\
100\end{array}$ & $\begin{array}{l}4302.11 \\
4304.404\end{array}$ & $\begin{array}{l}23237.9 \\
23225.48\end{array}$ & $(0 \mathrm{~W}) 1.1101$ & $\begin{array}{l}138_{3 / 2}-37066_{3 / 2} \\
1669 / 2-39847 / 2\end{array}$ & +.4 & -- \\
\hline $100 \mathrm{~d}$ & 4170.395 & 23971.78 & $\{(.569) .915$ & $\begin{array}{l}2709 / 2-01489 / 2 \\
138_{3 / 2}-3779_{1 / 2}\end{array}$ & $\left.\begin{array}{l}-.20 \\
-.05\end{array}\right\}$ & 9 & 2 & 4304.78 & 23223.5 & & $311_{5 / 2}-5440_{5 / 2}$ & $\begin{array}{c}+.01 \\
.0\end{array}$ & $\begin{array}{l}-- \\
-\end{array}$ \\
\hline & & & 2.051 . & & & & 15 & 4305.334 & 23220.47 & $(.261, .783)$ & $204_{3 / 2}-437 \gamma_{3 / 2}$ & .00 & - \\
\hline $\begin{array}{l}10 \\
10\end{array}$ & $\begin{array}{l}4173.978 \\
4175.186\end{array}$ & $\begin{array}{l}23951.21 \\
23944.27\end{array}$ & $(495)$ & $\begin{array}{l}194_{5 / 2}-4341_{5 / 2} \\
197_{1 / 2}-4370_{3 / 2}\end{array}$ & $\begin{array}{l}-.01 \\
-.02\end{array}$ & -- & $\begin{array}{l}4 \\
9\end{array}$ & $\begin{array}{l}4308.802 \\
4309.474\end{array}$ & $\begin{array}{l}23201.78 \\
23198.16\end{array}$ & & $8 / 2-49867 / 2$ & $\begin{array}{l}-.01 \\
-.02\end{array}$ & -- \\
\hline 10 & 4176.540 & 23936.51 & & $\begin{array}{l}19 / 1 / 2-43803 / 2 \\
150_{7 / 2}-3899_{9 / 2}\end{array}$ & $\begin{array}{l}-.02 \\
-.03\end{array}$ & -- & $2 \mathrm{~h}$ & 4313.31 & 23177.5 & & $\begin{array}{l}2.515 / 2-40507 / 2 \\
341_{9 / 2}-5737_{7 / 2}\end{array}$ & $\begin{aligned}-.02 \\
.0\end{aligned}$ & -- \\
\hline 6 & 4176.867 & 23934.64 & & $204_{3 / 2}-4441_{1 / 2}$ & +.04 & -- & 15 & 4314.578 & 23170.72 & $(.121, .359$ & $217_{7 / 2}-4.4947 / 2$ & .00 & -- \\
\hline 2 & 4177.419 & 23931.48 & $\ldots$ & $2887 / 2-52749 / 2$ & +.05 & -- & & & & $.596, .833)$ & & & \\
\hline 3 & 4178.599 & & - n & $280_{5 / 2}-51957 / 2$ & +.02 & -- & 20 & 4315. 71 & 23164.7 & $(.293)$ & $142_{7 / 2}-3738_{5 / 2}$ & +.1 & -- \\
\hline 3 & 4179.73 & $\begin{array}{l}23918.2 \\
23909.3\end{array}$ & $-\cdots$ & $319_{7 / 2}-5589_{7 / 2}$ & .0 & -- & 4 & 4316.18 & & & $301_{1 / 2}-5329_{3 / 2}$ & -.1 & -- \\
\hline${ }^{2}$ & $\begin{array}{l}4181.29 \\
4182.22\end{array}$ & $\begin{array}{l}23909.3 \\
23904.0\end{array}$ & $--\cdot$ & $3069 / 2-545511 / 2$ & .0 & $\overline{2} \overline{3}$ & 5 & 4317.11 & $\begin{array}{l}23157.2 \\
23154.1\end{array}$ & & $273_{11 / 2}-5040_{9 / 2}$ & +.1 & -. \\
\hline $\begin{array}{l}(1) \\
100\end{array}$ & $\begin{array}{l}4182.22 \\
4182.902\end{array}$ & $\begin{array}{l}23904.0 \\
23900.11\end{array}$ & $(.106, .31$ & $\begin{array}{l}239_{11 / 2}-4785_{11 / 2} \\
157_{5 / 2}-3967_{5 / 2}\end{array}$ & $\begin{array}{l}+.1 \\
+.01\end{array}$ & 23 & $\begin{array}{l}2 \mathrm{~h} \\
2 \mathrm{~h}\end{array}$ & $\begin{array}{l}4317.68 \\
4317.93\end{array}$ & & & $\begin{array}{l}298_{5 / 2}-5.5966_{3 / 2} \\
280_{5 / 2}-5118_{3 / 2}\end{array}$ & $\begin{array}{r}.0 \\
+\quad 1\end{array}$ & -- \\
\hline & & & $.522) .79$ & $1015 / 22 \quad 00015 / 2$ & & & 10 & 4318.585 & 23149.21 & & $272_{9 / 2}-50317 / 2$ & -.14 & $\cdots$ \\
\hline & & & $\begin{array}{l}1.002,1.212, \\
1.410,1.613 .\end{array}$ & & & & 15 & 4319. 531 & 23144.15 & $(.093$ & $\begin{array}{l}2755_{9 / 2}-5066_{11 / 2} \\
146_{5 / 2}-37 \gamma 6_{7 / 2}\end{array}$ & $\begin{array}{l}+.01 \\
-.04\end{array}$ & -- \\
\hline $\begin{array}{r}100 \\
2 \mathrm{~h}\end{array}$ & $\begin{array}{l}4183.056 \\
4184.48\end{array}$ & & (0w) $1.304 \mathrm{w}_{\ldots}$. & $146_{5 / 2}-3852_{7 / 2}$ & -.05 & -- & & & & $.464)$ & & & \\
\hline $2 \mathrm{~h}$ & $\begin{array}{l}4104.40 \\
4185.72\end{array}$ & $\begin{array}{l}23891.1 \\
23884.0\end{array}$ & & $332_{5 / 2}-5717_{7 / 2}$ & .0 & -- & 3 & 4324. 33 & $\begin{array}{l}231.18 .4 \\
23093.12\end{array}$ & & $261_{3 / 2}$ - $40255_{3} / 2$ & -.1 & -- \\
\hline 5 & 4187.909 & 23871.53 & & $\begin{array}{l}194_{5 / 2}-433443 / 2 \\
138_{3 / 2}-3760_{5 / 2}\end{array}$ & $\begin{array}{r}.0 \\
-01\end{array}$ & -- & $\begin{array}{r}3 \\
2 h\end{array}$ & $\begin{array}{l}4329.076 \\
4331.14\end{array}$ & $\begin{array}{l}23093.12 \\
23082.1\end{array}$ & & $\begin{array}{l}3069 / 2-53737 / 2 \\
311 / 2-5426\end{array}$ & -.01 & -- \\
\hline & 4188.441 & 23868.50 & & $\begin{array}{l}138_{3 / 2}-5 / 085 / 2 \\
306_{9 / 2}-5451_{7 / 2}\end{array}$ & $\begin{array}{l}-.01 \\
-.01\end{array}$ & -- & $\begin{array}{r}2 \mathrm{n} \\
3\end{array}$ & 4331.365 & 23080.91 & & $298_{5 / 2}-5288_{5 / 2}$ & -.05 & -. \\
\hline $3 \mathrm{Hl}$ & 4191.92 & 23848.7 & & $3452_{7 / 2}-58369 / 2$ & .0 & -. & 80 & 4332.25 & 23076.2 & (.106) $1.208 \mathrm{D}$ & $146_{5 / 2}-3769_{5 / 2}$ & .0 & 19 \\
\hline 50 & 4194.668 & 23833.07 & (.132) $1.258 \mathrm{~A}$ & $173_{7 / 2}-4116_{7 / 2}$ & -.02 & -. & 9 & 4335.860 & 23056.99 & $(.037, .112$, & $298_{5 / 2}-5285_{7 / 2}$ & -.07 & .. \\
\hline 10 & 4195.741 & 23826.97 & $\begin{array}{l}(.108, .323) \\
.885,1.100\end{array}$ & $204_{3 / 2}-4430_{5 / 2}$ & -.03 & -- & 8 & 4339.68 & & $.187) 1.478 \mathrm{~B}$. & $166_{9 / 2}-3965_{9 / 2}$ & .0 0 & \\
\hline & 4203,308 & 23784.08 & & $273_{11 / 2}-5102_{9 / 2}$ & & & 1 & 4345.32 & 23006.8 & & $3143 / 2-54463 / 2$ & .0 & $\cdots$ \\
\hline 30 & 4204.525 & 23777.19 & (w) 1.05 & $\begin{array}{l}273_{11 / 2}-51029 / 2 \\
221_{9 / 2}-4583_{7 / 2}\end{array}$ & $\begin{array}{r}.00 \\
+.05\end{array}$ & -- & 2 & $\begin{array}{l}4345.505 \\
4352.46\end{array}$ & $\begin{array}{l}23005.81 \\
22969.1\end{array}$ & & $\begin{array}{l}266_{5 / 2}-1966_{3 / 2} \\
271 / 5011 / 2\end{array}$ & -.01 & -- \\
\hline 30 & 4213.266 & 23727.87 & & $261_{3 / 2}-4985_{1 / 2}$ & -.09 & -. & $\begin{array}{r}4 \\
10\end{array}$ & 4357.06 & 22944.8 & & $\begin{array}{l}271_{7 / 2}-5011_{5 / 2} \\
247_{7 / 2}-4766_{7 / 2}\end{array}$ & $\begin{array}{r}-.1 \\
.0\end{array}$ & -- \\
\hline 2 & 4214.974 & 23718.25 & $\{(w) 1.2$ & $271_{7 / 2}-50867 / 2$ & .00 & 9 & 100 & 4358.69 & 22936.2 & $(.088, .262$ & $115_{5 / 2}-3452_{7 / 2}$ & -.1 & -- \\
\hline 15 & 4215.500 & 23715.30 & & $\begin{array}{l}2243 / 2-46191 / 2 \\
2759 / 2-5122_{7 / 2}\end{array}$ & $\begin{array}{l}-.03 \\
-.03\end{array}$ & -- & & & & $\begin{array}{l}.437) \text { 1.188, } \\
1.363,1.533\end{array}$ & & & \\
\hline 200 & 4221.079 & 23683.95 & (.277) 1.130 & $115_{5 / 2}-8526_{5 / 2}$ & -.03 & $\cdots$ & & & & $1.707,1.880$. & & & \\
\hline & & & $\begin{array}{l}1.238,1.344 \\
1.452,1.560 .\end{array}$ & & & & $\begin{array}{r}7 \\
10\end{array}$ & $\begin{array}{l}4359.310 \\
4360.375\end{array}$ & $\begin{array}{l}22932.96 \\
22927.36\end{array}$ & & $\begin{array}{l}244_{5 / 2}-47355_{1 / 2} \\
204_{3 / 2}-4841_{5 / 2}\end{array}$ & $\begin{array}{r}.00 \\
-.02\end{array}$ & -- \\
\hline 2 & 4222.500 & 23675.98 & - $10-102$, 10000 & $28.57 / 2-52.21_{5 / 2}$ & .00 & -. & 4 & 4361.26 & 22922.7 & & $221_{9 / 2}-45988_{11 / 2}$ & +.1 & - \\
\hline 15 & 4224.163 & 23666.66 & & $204_{3 / 2}-441 .+3 / 2$ & -.06 & -. & 6 & 4363.37 & 22911.6 & & $266_{5 / 2}-4957_{5 / 2}$ & -.1 & $\ldots$ \\
\hline 2000 & 4227.46 & 23618.2 & $\begin{array}{l}(.133, .394, \\
.657) 1.292, \\
1.596,1.855\end{array}$ & $1895_{5 / 2}-4253_{7 / 2}$ & .0 & $\cdots$ & $\begin{array}{r}10 \\
9\end{array}$ & $\begin{array}{l}4364.13 \\
4364.851\end{array}$ & $\begin{array}{l}22907.6 \\
22903.85\end{array}$ & $\begin{array}{l}(.333 w) \\
(.070, .209, \\
.347 .)\end{array}$ & $\begin{array}{l}305_{11 / 2}-5346_{11 / 2} \\
2805 / 2-509.9_{5 / 2}\end{array}$ & $\begin{array}{l}.0 \\
-.01\end{array}$ & -. \\
\hline & & & $2.114,2.372$, & & & & 1 & 4366. 35 & $\begin{array}{l}22896.0 \\
22880.5\end{array}$ & & $341_{9 / 2}-57099_{7 / 2}$ & .0 & -- \\
\hline 20 & 4232.956 & 23617.50 & $\begin{array}{l}(.626 . \\
.270) .162\end{array}$ & $280_{5 / 2}-516_{4 i 7 / 2}$ & .00 & -. & & $436 \% .58$ & & $\begin{array}{l}(.621, .776, \\
.929) .862, \\
1.014,1.168,\end{array}$ & $163_{11 / 2}-391 \%_{11 / 2}$ & & $\ldots$ \\
\hline 10 & 4233.275 & $\begin{array}{l}23615.72 \\
23609.3\end{array}$ & & $173_{7 / 2}-40947 / 2$ & +.01 & -- & & & & $1.323,1.476$ & & & \\
\hline $\begin{array}{r}6 \\
10\end{array}$ & $\begin{array}{l}4234.43 \\
4234.95\end{array}$ & $\begin{array}{l}23609.3 \\
23606.4\end{array}$ & & & +.1 & -. & 2 & $\begin{array}{l}4369.64 \\
4369.77\end{array}$ & $\begin{array}{l}22878.7 \\
22879.1\end{array}$ & \}$(.581 \mathrm{w})$ & $\left\{\begin{array}{c}273^{\prime}{ }^{\prime / 2}-5026_{3 / 2} \\
273^{\prime \prime}{ }_{1 / 2}-5026_{3 / 2}\end{array}\right.$ & $\begin{array}{l}-1 \\
+1\end{array}$ & 20 \\
\hline $\begin{array}{l}10 \\
20\end{array}$ & 4236.237 & 23599.20 & $(1.448) .252$ & $\begin{array}{r}20447 / 2-4405_{5 / 2} \\
1643 / 2-3992_{3 / 2}\end{array}$ & $\begin{array}{c}.0 \\
-.04\end{array}$ & -- & $\begin{array}{l}4 \\
5\end{array}$ & 4373. 202 & 22860.11 & $(.720)$ & $\begin{array}{r}2 / 3,1 / 2-50263 / 2 \\
2043 / 2-43943 / 2\end{array}$ & $\begin{array}{l}+.1 \\
-.01\end{array}$ & $\ldots$ \\
\hline & 4238.016 & & 1.227 & & & & 2 & 4377.056 & 22839.98 & & $319_{7 / 2}-5482_{5 / 2}$ & -.05 & - \\
\hline $\begin{array}{r}7 \\
15\end{array}$ & $\begin{array}{l}4238.016 \\
4238.586\end{array}$ & $\begin{array}{l}23589.30 \\
23586.13\end{array}$ & $(.074) \ldots \ldots$ & $298_{5 / 2}-5338_{5 / 2}$ & -.05 & -- & 3 & 4377.44 & $\begin{array}{l}22838.0 \\
208 ? .0\end{array}$ & & $2887 / 2-51647 / 2$ & .0 & $\ldots$ \\
\hline $\begin{array}{r}15 \\
2\end{array}$ & 4239.956 & $\begin{array}{l}235 \times 6.13 \\
23578.51\end{array}$ & $(.213)_{-}$ & $194: / 2-430.45 / 2$ & .09 & -- & 1 & 4380.45 & $\begin{array}{l}22822.3 \\
22819.4\end{array}$ & & $3069 / 2-53466_{11 / 2}$ & +.1 & \\
\hline 40 & 4241.16 & 23571.8 & (?) .964 & $\begin{array}{l}231_{5 / 2}-4678_{3 / 2} \\
172 / 2-4081_{1 / 2}\end{array}$ & -.06 & 17 & (10) & 4381.00 & 22819.4 & & $\begin{array}{l}30511 / 2-53379 / 2 \\
275\end{array}$ & $\begin{array}{r}.0 \\
-10\end{array}$ & 7 \\
\hline 50 & 4241.42 & 23570.4 & (?) .766 & $\begin{array}{l}172_{1 / 2}-40811_{1 / 2} \\
172_{1 / 2}-4080_{3 / 2}\end{array}$ & $\begin{array}{l}-.1 \\
-.2\end{array}$ & 17 & 10 & 4385.443 & 22796.30 & $\{(.108, .324)$ & $194_{5 / 2}-42255_{3 / 2}$ & .00 & $\cdots$ \\
\hline 2 & 4241. 806 & 23568.23 & 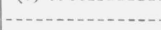 & $217_{7 / 2}-45849 / 2$ & +.06 & -. & 2 & 4386.14 & 22792.7 & & $3526_{5 / 2}-5806_{7 / 2}$ & 0 & - \\
\hline $\begin{array}{r}2 \\
20\end{array}$ & 4243.85 & $\begin{array}{l}23556.8 \\
23555.4\end{array}$ & & $217_{7 / 2}-458.9_{5 / 2}$ & +.2 & -- & 10 & 4387.404 & 22786.11 & $(.160, .428)$ & $221_{8 / 2}-44947 / 2$ & +.03 & \\
\hline $\begin{array}{r}20 \\
40 \mathrm{hl}\end{array}$ & $\begin{array}{l}4244.12 \\
4246.820\end{array}$ & $\begin{array}{l}23555.4 \\
23540.40\end{array}$ & $\begin{array}{l}(.248) \\
(418)\end{array}$ & $138_{3 / 2}-3738_{5 / 2}$ & +.1 & -- & (2) & 4388.52 & $\begin{array}{l}22780.3 \\
22765.68\end{array}$ & & $288_{7 / 2}-51595 / 2$ & +.1 & 13 \\
\hline $4 \mathrm{~h}$ & 4247.679 & 23535.64 & & $\left\{\begin{array}{r}3452_{7 / 2}-58067 / 2 \\
261_{3 / 2}-4966_{3 / 2}\end{array}\right.$ & $\begin{array}{l}+.01 \\
-.04\end{array}$ & -- & 100 & 4391.342 & 22765.68 & . 224$) 1.747$ & $1179 / 2-34587 / 2$ & -.05 & $\cdots$ \\
\hline 2 & 4248. 357 & 23531.88 & & $298_{5 / 2}-5939.97 / 2$ & +.08 & - & 3 & & & & $304_{3 / 2}-5328_{1 / 2}$ & -.04 & -- \\
\hline 2 & 4255. 10 & 23494,6 & & $\begin{array}{l}306_{9 / 2}-5417_{7 / 2} \\
2315-46645 / 2\end{array}$ & -.04 & -- & $\begin{array}{l}40 \\
80\end{array}$ & $\begin{array}{l}4392.45 \\
4394.30\end{array}$ & $\begin{array}{l}22760.0 \\
22750.3\end{array}$ & $\begin{array}{l}\text { (?) } .988,1.075 \\
\{(.437) 1.053,\end{array}$ & $146_{5 / 2}-3738_{5 / 2}$ & 0 & -- \\
\hline 2 & $\begin{array}{l}4255.33 \\
425.749\end{array}$ & $\begin{array}{l}23493.3 \\
23491.01\end{array}$ & & $298_{5 / 2}-5.3299_{3 / 2}$ & $\begin{array}{l}.0 \\
.0\end{array}$ & -- & 50 & 4394.46 & 22749.5 & $\left\{\begin{array}{l}\text { \{. } \\
1.928\end{array}\right.$ & $151_{1 / 2}-3791_{3 / 2}$ & $\left\{\begin{array}{l}+.3 \\
-.5\end{array}\right.$ & 24 \\
\hline 200 & $\begin{array}{l}4255.749 \\
4257.60\end{array}$ & $\begin{array}{l}23491.01 \\
23480.8\end{array}$ & & $173_{7 / 2}-4082_{5 / 2}$ & .00 & -- & 10 & 4396. 089 & 22741.10 & & $221_{9 / 2}-4490_{7 / 2}$ & -.01 & -- \\
\hline 250 & 4257.60 & 23480.8 & $\begin{array}{l}(.176, .526, \\
875) 1.043,\end{array}$ & $142_{7 / 2}-8769_{5 / 2}$ & .0 & -- & 30 & 4396.802 & 22737.41 & $\begin{array}{l}(.184, .550) \\
.784,1.151\end{array}$ & $164_{3 / 2}-3906_{5 / 2}$ & .00 & -- \\
\hline & & & $\begin{array}{l}\text { 1.393, 1.745, } \\
2.096,2.447\end{array}$ & & & & $\begin{array}{r}8 \\
40\end{array}$ & $\begin{array}{l}4399.80 \\
4402.600\end{array}$ & $\begin{array}{l}22721.9 \\
22707.47\end{array}$ & (.662) .700 & $\begin{array}{l}271_{7 / 2}-4986_{7 / 2} \\
150_{7 / 2}-37 \% 6_{7 / 2}\end{array}$ & $\begin{array}{l}-.2 \\
+.01\end{array}$ & -- \\
\hline $\begin{array}{c}(1) \\
3\end{array}$ & $\begin{array}{l}4258.88 \\
4259.880\end{array}$ & $\begin{array}{l}23473.8 \\
23468.23\end{array}$ & & $\begin{array}{l}244_{5 / 2}-4789_{5 / 2} \\
204_{3 / 2}-43945 / 2\end{array}$ & $\begin{array}{l}.0 \\
-.02\end{array}$ & 23 & & & & $\begin{array}{l}881,1.062, \\
1.243,1.424\end{array}$ & & & \\
\hline 6 & 4260.91 & 23462.6 & & $1507 / 2-38527 / 2$ & .0 & $\ldots$ & 5 & 4403. 683 & 22701.88 & & $272_{9 / 2}-4986_{7 / 2}$ & +.01 & \\
\hline 3 & 4261,86 & 23457.3 & & $341_{9 / 2}-5765_{9 / 2}$ & .0 & -- & 1 & & & & $2243 / 2-4512_{3 / 2}$ & -.2 & $\begin{array}{l}-- \\
--\end{array}$ \\
\hline $\begin{array}{l}0 \\
3\end{array}$ & 4263.35 & 23449.1 & & $338_{7 / 2}-5798_{0 / 2}$ & +.1 & -- & 20 & 4406.30 & 22688.4 & (.883) .150 & $172_{1 / 2}-3992_{3 / 2}$ & -.1 & - \\
\hline 3 & 4263.76 & 2344 & & $266_{5 / 2}-5011_{5 / 2}$ & +.2 & -- & 30 & 4406.47 & 22687.5 & $\{(000) 1.25$ & $163_{11 / 2}-38999 / 2$ & -.1 & - \\
\hline $2 \mathrm{~h}$ & 4264.73 & 23441 & & $280 \varepsilon / 2-5147_{5 / 2}$ & +.1 & $-\cdot$ & & & & & $285_{7 / 2}-51222_{7 / 2}$ & & - \\
\hline 5 & 4268.71 & & & $\begin{array}{l}261_{3 / 2}-4957_{5 / 2} \\
273_{11 / 2}-5066_{11 / 2}\end{array}$ & $\begin{array}{r}.0 \\
+\quad 1\end{array}$ & -- & 3 & 4408.64 & 22676.4 & & $\begin{array}{l}288_{7 / 2}-5148_{9 / 2} \\
244_{5 / 2}-4710_{5 / 2}\end{array}$ & $+^{\circ}$ & -- \\
\hline 3 & 4269. & $2341:$ & & $\begin{array}{l}273_{11 / 2}-5066_{11 / 2} \\
313_{9 / 2}-5481_{9 / 2}\end{array}$ & $\begin{array}{r}+.1 \\
.0\end{array}$ & -- & $2 \mathrm{~h}$ & 4410.39 & 22667.4 & & $\begin{array}{l}2445 / 2-4 / 10_{5 / 2} \\
288 i / 2-5147_{5 / 2}\end{array}$ & $\begin{array}{l}+.2 \\
+.1\end{array}$ & -. \\
\hline 20 & 4269.94 & $2341:$ & & $285_{7 / 2}-5195_{7 / 2}$ & +.1 & - & (1) & & & & $311_{5 / 2}-53843 / 2$ & -.1 & 23 \\
\hline 10 & 4274.34 & 23388.9 & & $305_{11 / 2}-539411 / 2$ & +.1 & -. & 1 & 4413.500 & 22651.39 & & $2857 / 2-51199 / 2$ & +.03 & 5 \\
\hline 3 & 4279.185 & 23362.36 & & $314_{3 / 2}-54825 / 2$ & -.04 & -- & 80 & 4415.822 & 22639.48 & $(0 \mathrm{w}) .987 \mathrm{C} \dagger-$ & $150_{7 / 2}-3769_{5 / 2}$ & +.01 & -. \\
\hline 6 & 4280.59 & 23354.7 & & $\begin{array}{l}9 / 2-50867 / 2 \\
5 / 2-4650_{5 / 2}\end{array}$ & $\begin{array}{r}.0 \\
+.1\end{array}$ & -- & $\begin{array}{r}8 \\
10\end{array}$ & $\begin{array}{l}4417.22 \\
4417.33\end{array}$ & $\begin{array}{l}226: \\
226\end{array}$ & & $151_{1 / 2}-3 \gamma 79_{1 / 2}$ & $\left\{\begin{array}{l}+.2 \\
-.3\end{array}\right.$ & 24 \\
\hline & & & & $\begin{array}{l}231_{5 / 2} \text { - } 4650_{5 / 2} \\
244_{5 / 2}-4777_{3 / 2}\end{array}$ & $\begin{array}{l}.1 \\
+.2\end{array}$ & - & $2 \mathrm{~h}$ & 4419.86 & & & $313_{9 / 2}-5401_{7 / 2}$ & -.1 & \\
\hline 2 & 4281.664 & 23348.83 & & $163_{11 / 2}-3965_{9 / 2}$ & -.01 & -- & 2 & 4423.810 & 22598.60 & & $304_{3 / 2}-5312_{3 / 2}$ & +.01 & -. \\
\hline 10 & 4282.734 & 23343.00 & & $164_{3 / 2}-396 \gamma_{5 / 2}$ & -.01 & -- & 2 & 4425.762 & 22588.63 & & $266_{5 / 2}-4925_{3 / 2}$ & +.04 & \\
\hline $1 \mathrm{~h}$ & 4285.55 & 23327.6 & & $311_{5 / 2}-54517 / 2$ & -.2 & -- & (4) & 4425.95 & 22587.7 & & $3387 / 2-5641_{9 / 2}$ & +.1 & 13 \\
\hline 10 & 4286.463 & 23322.69 & (?) $1.267 \mathrm{~h}$ & $271_{7 / 2}-5046_{5 / 2}$ & +.02 & -- & 2 & 4427.67 & 22578.9 & & $244 \varepsilon / 2-4700_{3 / 2}$ & .0 & -- \\
\hline 100 & 4291.17 & 23297.1 & $\begin{array}{l}(.068, .208) \\
1.420,1.579\end{array}$ & $1669 / 2-3991_{11 / 2}$ & .0 & -- & $\begin{array}{r}7 \\
2 h\end{array}$ & $\begin{array}{l}4427.951 \\
4428.864\end{array}$ & $\begin{array}{l}22577.47 \\
22572.81\end{array}$ & & $\begin{array}{l}166_{9 / 2}-3919_{11 / 2} \\
298_{5 / 2}-5237_{7 / 2}\end{array}$ & $\begin{array}{l}+.01 \\
+.07\end{array}$ & -- \\
\hline & & & $\begin{array}{l}1.720,1.079, \\
1.740\end{array}$ & & & & 41 & 4432.846 & 22552.53 & & $311_{5 / 2}-53 \gamma_{37 / 2}$ & +.15 & -- \\
\hline
\end{tabular}


TABle 2. Classified lines of Re $\mathrm{I}$-Continued

\begin{tabular}{|c|c|c|c|c|c|c|c|c|c|c|c|c|c|}
\hline Int. & $\lambda_{\mathrm{vac}}$ & $\sigma_{\mathrm{vac}}$ & Zeeman effect & Transition & $\mathrm{O}-\mathrm{C}$ & Notes & Int. & $\lambda_{\mathrm{vac}}$ & $\sigma_{\mathrm{vac}}$ & Zeeman effect & Transition & $\mathrm{O}-\mathrm{C}$ & Notes \\
\hline 3 & $\underset{4436.614}{A}$ & $\begin{array}{c}K \\
22533.38\end{array}$ & & $217_{7 / 2}-4430_{5 / 2}$ & +.05 & $\ldots$ & 200 & $\begin{array}{c}A \\
4605.73\end{array}$ & $\begin{array}{c}K \\
21706.0\end{array}$ & $(0 \mathrm{w}) 1.312 \mathrm{~B}$ & $194_{5 / 2}-4116_{7 / 2}$ & 0 & \\
\hline 20 & 4440.44 & 22514.0 & (?) 1.25 & $1737 / 2-39847 / 2$ & $\begin{array}{l}+.10 \\
+.1\end{array}$ & - & 3 & 4607.80 & 21696.3 & & $\begin{array}{l}1945 / 2-4107 / 2 \\
272_{9 / 2}-48857 / 2\end{array}$ & .0 & -- \\
\hline $2 \mathrm{~h}$ & 4445.97 & 22486.0 & & $285_{7 / 2}-5102_{9 / 2}$ & +.2 & -. & 4 & 4608.802 & 21691.53 & & $304_{3 / 2}-5221_{5 / 2}$ & +.02 & -- \\
\hline 2 & 4448.115 & 22475.12 & & $273^{\prime}{ }_{1 / 2}-4985_{1 / 2}$ & -.01 & - & $1 \mathrm{~h}$ & 4609.81 & 21686.8 & & $244_{5 / 2}-4611_{5 / 2}$ & .0 & $\ldots$ \\
\hline 2 & 4448. 259 & 22474. 39 & & $273^{\prime \prime}{ }_{1 / 2}-4385_{1 / 2}$ & +.06 & -. & 5 & 4613.95 & 21667.3 & & $305_{11 / 2}-5222_{13 / 2}$ & .0 & - \\
\hline 2 & $\begin{array}{l}4453.26 \\
4453.92\end{array}$ & $\begin{array}{l}22449.2 \\
22445.8\end{array}$ & & $217_{7 / 2}-44222_{9 / 2}$ & .0 & -- & 50 & 4614. 66 & 21664.0 & (.000) $1.248 \mathrm{~h}$ & $173_{7 / 2}-3899_{9 / 2}$ & +.1 & -. \\
\hline $\begin{array}{l}20 \\
50\end{array}$ & $\begin{array}{l}4453.92 \\
4454.62\end{array}$ & $\begin{array}{l}22445.8 \\
22442.3\end{array}$ & $(.257) .89$ & $\begin{array}{r}3592_{9 / 2}-58369 / 2 \\
146_{5 / 2}-3706_{3 / 2}\end{array}$ & $\begin{array}{l}-.1 \\
+.1\end{array}$ & - & $\begin{array}{l}30 \\
80\end{array}$ & $\begin{array}{l}4616.57 \\
4621.38\end{array}$ & 21655.1 & & $221_{9 / 2}-43817 / 2$ & +.1 & $\overline{2} \overline{6}$ \\
\hline 年 & & & $1.415,1.9$ & $280_{5} / 2-.5046_{5}^{\prime \prime}$ & 1.1 & & 80 & 4621.38 & 21632.5 & $\begin{array}{l}(.439, .724) \\
1.003,1.274,\end{array}$ & $217_{7 / 2}-4340_{7 / 2}$ & .0 & 20 \\
\hline 6 & 4456. 668 & 22431. 99 & & $271_{7 / 2}-4957_{5 / 2}$ & +.01 & -- & (1h) & 4624.73 & 21616.8 & & $285_{7 / 2}-5015_{5 / 2}$ & +.1 & 23 \\
\hline 2 & 4457.47 & 22428.0 & & $304_{3 / 2}-5296_{3 / 2}$ & +.1 & -. & 30 & 4625.96 & 21611. 1 & & $157_{5 / 2}-3738_{5 / 2}$ & $\begin{array}{l}\text { T. } \\
+.1\end{array}$ & 20 \\
\hline 3 & 4459.405 & 22418.22 & & $239_{11 / 2}-4697_{9 / 2}$ & -.03 & - & 1 & 4628. 76 & 21598.0 & & $314_{3 / 2}-5305_{5 / 2}$ & T. 1 & $\begin{array}{l}-- \\
--\end{array}$ \\
\hline 2 & 4464. 627 & 22392.00 & & $285_{7 / 2}-509.3_{5 / 2}$ & -.05 & -. & 7 & 4630.24 & 21591. 1 & & $288_{7 / 2}-5040_{9 / 2}$ & .0 & - \\
\hline 5 & 4465.834 & 22385.95 & & $1945 / 2-41847 / 2$ & -.01 & -. & 80 & 4630.82 & 21588.4 & (?) 1.594 & $164_{3 / 2}-3791_{3 / 2}$ & .0 & $\ldots$ \\
\hline 15 & 4467.544 & 22377.38 & & $2477 / 2-4710_{5 / 2}$ & -.01 & - & 3 & 4635.78 & 21565.3 & & $231_{5 / 2}-4472_{3 / 2}$ & .0 & .. \\
\hline 40 & 4467. 924 & 22375.47 & & $166_{9 / 2}-38999_{9 / 2}$ & +.02 & -- & $1 \mathrm{~h}$ & 4640.63 & 21542.8 & & $280_{5 / 2}-4957_{5 / 2}$ & 0 & - \\
\hline 2 & 4472. 06 & 22354.8 & & $304_{3 / 2}-5288_{5 / 2}$ & +.1 & -. & ih & 4640.94 & 21541.3 & & $332_{5 / 2}-54825 / 2$ & -.1 & -- \\
\hline 4 & 4473. 23 & 22348.9 & & $275_{9 / 2}-4986_{7 / 2}$ & .0 & -- & 2 & 4642.68 & 21533.3 & & $261_{3 / 2}-4765_{1 / 2}$ & +.2 & - \\
\hline 80 & 4475.082 & 22339.68 & $(.073, .217$, & $1737 / 2-3967_{5 / 2}$ & -.02 & $\therefore$ & 20 & 4644.95 & 21522.7 & & $266_{5 / 2}-4818_{7 / 2}$ & -.1 & -. \\
\hline & & & $\begin{array}{l}.362) 1.328, \\
1.475, \mathbf{1 . 6 2 1} .\end{array}$ & & & & $\begin{array}{r}10 \\
4\end{array}$ & $\begin{array}{l}4647.46 \\
4648.56\end{array}$ & $\begin{array}{l}21511.1 \\
21506.0\end{array}$ & & $\begin{array}{c}263_{13 / 2}-4785_{11 / 2} \\
28888_{7 / 2}-5039_{9 / 2}\end{array}$ & $\begin{array}{r}+.1 \\
.0\end{array}$ & -- \\
\hline 6 & 4476.78 & 22331.2 & & $278_{3 / 2}-5015_{5 / 2}$ & .0 & .- & $1 \mathrm{~h}$ & 4649.19 & 21503. 1 & & $\begin{array}{l}20007 / 2-50399 / 2 \\
3776_{7 / 2}-5926_{5 / 2}\end{array}$ & .0 & -- \\
\hline 3 & 4477.642 & 22326. 92 & & $285_{7 / 2}-5086_{7 / 2}$ & +.05 & $\ldots$ & 3 & 4649. 70 & 21500.8 & & $288_{7 / 2}-5031_{7 / 2}$ & .0 & -- \\
\hline 30 & 4477.986 & 22325. 20 & $(.091)$ & $1737 / 2-39555_{9 / 2}$ & +.03 & - & $3 \mathrm{~h}$ & 4650.825 & 21495.54 & & $333_{11 / 2}-5481_{9 / 2}$ & -.01 & - \\
\hline 60 & 4478.386 & 22323.20 & $\begin{array}{l}(.079, .242) \\
1.068,1.232,\end{array}$ & $1507 / 2-3738_{5 / 2}$ & -.02 & - & $\mathrm{1h}_{4}$ & $\begin{array}{l}4651.20 \\
4651.82\end{array}$ & $\begin{array}{l}21493.8 \\
21491.0\end{array}$ & & $314_{3 / 2}-5296_{3 / 2}$ & $\begin{array}{l}.0 \\
.0\end{array}$ & -- \\
\hline & & & $1.375, \mathbf{1 . 5 5}$ & $\int 231_{5 / 2}-45467 / 2$ & -.04 & -- & 100 & 4652.33 & 21488.6 & $(.087, .254)$ & $\begin{array}{l}314_{3 / 2}-52955_{3 / 2} \\
194_{5 / 2}-40947 / 2\end{array}$ & .0 & -- \\
\hline 10 & 4481.443 & 22307.98 & & $\left\{\begin{array}{l}244_{5 / 2}-467_{3 / 2} \\
y_{3}\end{array}\right.$ & .00 & -- & & & & $\begin{array}{l}1.44 \\
1.79\end{array}$ & & & \\
\hline $3 \mathrm{~h}$ & 4486.58 & 22282.4 & & $278_{3 / 2}-5011_{5 / 2}$ & -.2 & -. & 2 & 4652.77 & 21486.6 & & $2885_{5 / 2}-5034_{5 / 2}$ & +.2 & -- \\
\hline 5 & 4486.991 & 22280.39 & & $280_{5 / 2}-5031_{7 / 2}$ & +.01 & -- & 10 & 4654.53 & 21478.4 & & $2885_{5 / 2}-5033_{3 / 2}$ & .0 & - \\
\hline 15 & 4496. 432 & 22233.62 & (?) $.79 t$ & $197_{1 / 2}-4199_{1 / 2}$ & -.03 & -- & $2 \mathrm{~h}$ & 4656.29 & 21470.3 & & $164_{3 / 2}-3779_{1 / 2}$ & -.1 & -. \\
\hline 10 & 4498.378 & 22224.00 & & $244_{5 / 2}-46645 / 2$ & -.02 & -. & 10 & 4656.47 & 21469.5 & & $2888_{7 / 2}-5035_{7 / 2}$ & .0 & -- \\
\hline $\begin{array}{l}3 \\
4\end{array}$ & $\begin{array}{l}4499.04 \\
4502.52\end{array}$ & $\begin{array}{l}22220.7 \\
22203.6\end{array}$ & & $\begin{array}{l}263_{13 / 2}-4856_{11 / 2} \\
311_{5 / 2}-5338_{5 / 2}\end{array}$ & $\begin{array}{r}.0 \\
-.1\end{array}$ & -- & 10 & 4660.51 & 21450.8 & & $\left\{\begin{array}{r}2888_{7 / 2}-5034_{5 / 2} \\
244,57^{7}\end{array}\right.$ & -.1 & -- \\
\hline 4 & 4504.11 & 22194.3 & & $3197 / 2-5417_{7 / 2}^{5}$ & .9 & & 9 & 4661.483 & 21446. 39 & & $\begin{array}{l}244_{5 / 2}-4587_{5 / 2} \\
301_{1 / 2}-5157_{3 / 2}\end{array}$ & $\begin{array}{l}-.1 \\
-.09\end{array}$ & $-\overline{5}$ \\
\hline 100 & 4507.035 & 22181.31 & $(.814)$ & $\left\{2885_{5 / 2}-5104_{5 / 2}\right.$ & -.07 & 21 & 60 & 4662.493 & 21441.75 & & $138_{3 / 2}-35266_{5 / 2}$ & -.07 & .. \\
\hline 60 & 4508.007 & 2217652 & (?) $.121, .418^{+}$ & ( $305_{11 / 2}-597 / 49 / 2$ & .00 & 25 & 8 & 4662. 99 & 21439.4 & & $270_{13 / 2}-4856_{11 / 2}$ & -.1 & -- \\
\hline $\begin{array}{r}60 \\
1\end{array}$ & $\begin{array}{l}4508.007 \\
4508.39\end{array}$ & $\begin{array}{l}22176.52 \\
22174.6\end{array}$ & (?) $.121, .418 \dagger$ & & -.03 & 21 & 5 & 4665. 217 & 21429.23 & & $2988_{5 / 2}-51227 / 2$ & -.03 & -- \\
\hline 500 & 4513.31 & 22150.5 & (.350) $1.967 \mathrm{~A}_{-}$ & $\begin{array}{r}217_{7 / 2}-4.3945 / 2 \\
20447 / 2-4259_{7 / 2}\end{array}$ & $\begin{array}{l}.0 \\
.0\end{array}$ & -- & $\begin{array}{l}6 \\
2\end{array}$ & $\begin{array}{l}4666.713 \\
4669.81\end{array}$ & $\begin{array}{l}21422.36 \\
21408.1\end{array}$ & & $\begin{array}{l}278_{3 / 2}-4925_{3 / 2} \\
272_{9 / 2}-4856_{11 / 2}\end{array}$ & $\begin{array}{l}-.01 \\
-.2\end{array}$ & -- \\
\hline 20 & 4514. 26 & 22145.8 & & $28887 / 2-5104_{5 / 2}$ & 0 & - & 9 & 4674. 304 & 21387.57 & & $\begin{array}{l}2329 / 2-403011 / 2 \\
239_{11 / 2}-45349 / 2\end{array}$ & -.00 & $\begin{array}{l}-- \\
--\end{array}$ \\
\hline 200 & 4516.638 & 22134.15 & $.800), .475$ & $2885_{5 / 2}-5098_{7 / 2}$ & -.14 & - & 2 & 4675,38 & 21382.6 & & $298_{5 / 2}-5118_{3 / 2}$ & .0 & - \\
\hline 1 & 4517.774 & 22128.58 & & $280_{5 / 2}-5015_{5 / 2}$ & +.03 & -- & 15 & 4679.476 & 21363.93 & & $\begin{array}{r}194_{5 / 2}-4082_{5 / 2} \\
319_{7 / 2}-5333_{7 / 2}\end{array}$ & $\begin{array}{l}-.01 \\
-.1\end{array}$ & -- \\
\hline 3 & 4518.570 & $\begin{array}{l}22128.08 \\
22124.69\end{array}$ & & $2729 / 2-4928_{11 / 2}$ & -.03 & -- & $2 \mathrm{~h}$ & 4681.89 & 21352.9 & & $\left\{\begin{array}{r}0197 / 2-53837 / 2 \\
3791_{3 / 2}-5926_{5 / 2}\end{array}\right.$ & $\begin{array}{r}-.1 \\
.0\end{array}$ & -- \\
\hline 80 & 4519.762 & 22118.85 & $(.975) .894$ & $2885_{5 / 2}-5097_{5 / 2}$ & -.14 & - & 60 & 4682.319 & 21350.96 & & $194_{5 / 2}-4080_{3 / 2}$ & .00 & 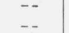 \\
\hline & & & $\begin{array}{l}1.291, \\
1.684 \ldots+\end{array}$ & & & & $\begin{array}{l}(5 \mathrm{~h}) \\
1 \mathrm{~h}\end{array}$ & $\begin{array}{l}4684.02 \\
4685.20\end{array}$ & $\begin{array}{l}21343.2 \\
21337.8\end{array}$ & & $\begin{array}{l}275_{9 / 2}-4885_{7 / 2} \\
319_{7 / 2}-5332_{9 / 2}\end{array}$ & $\begin{array}{l}-.1 \\
-.2\end{array}$ & 7 \\
\hline 400 & 4522.73 & 22104.3 & $\mathrm{P}-\mathrm{B}$ & $2888_{7 / 8}-5099_{9 / 2}$ & -.1 & & $(15)$ & $\begin{array}{l}4000.20 \\
4687.858\end{array}$ & $\begin{array}{l}21301.0 \\
21325.74\end{array}$ & & $\begin{array}{l}319_{7 / 2}-5332_{9 / 2} \\
273_{11 / 2}-4856_{11 / 2}\end{array}$ & $\begin{array}{l}-.2 \\
-.01\end{array}$ & $\overline{3}$ \\
\hline 100 & 4523.878 & 22098.72 & $\mathrm{P}-\mathrm{B}$ & $2888_{7 / 2}-5098_{7 / 2}$ & -.03 & 26 & $1 \mathrm{~h}$ & 4688.87 & 21321.1 & & $285_{7 / 2}-4986_{7 / 2}$ & .0 & - \\
\hline 80 & 452 ค. 009 & 22088.32 & $\mathrm{P}-\mathrm{B}$ & $28966_{3 / 2}-5105_{1 / 2}$ & .00 & & $4 \mathrm{~h}$ & 4691.45 & 21309.4 & & $266_{5 / 2}-4797_{5 / 2}$ & .0 & -. \\
\hline 10 & 4526.98 & 22083.6 & & $2888_{7 / 2}-5097_{5 / 2}$ & +.1 & -- & 1 & 4692.80 & 21303. 3 & & $324_{5 / 2}-5378_{7 / 2}$ & .0 & -- \\
\hline 60 & 4528.966 & 22073.90 & $\mathrm{P}-\mathrm{B}$ & $\left\{\begin{array}{r}2985 / 2-5187_{3 / 2} \\
2896-5104\end{array}\right.$ & -.15 & -- & 8 & 4693.38 & 21300.7 & & $306_{9 / 2}-519411 / 2$ & +.1 & -- \\
\hline 150 & 4529.950 & 22069.11 & $\mathrm{P}-\mathrm{B}$ & $\begin{array}{r}128966_{3 / 2}-51045 / 2 \\
28966_{3 / 2}-51033 / 2\end{array}$ & $\begin{array}{l}-.11 \\
-.07\end{array}$ & 26 & 30 & 4694.99 & 21293.6 & & $\begin{array}{l}221_{9 / 2}-43455 / 2 \\
1575-37063 / 2\end{array}$ & $\begin{array}{l}+.1 \\
+2\end{array}$ & -- \\
\hline 30 & 4530.889 & 22064.53 & & $221_{9 / 2}-4422_{9 / 2}$ & -.01 & -- & $2 \mathrm{~h}$ & 4698.08 & 21279.3 & & $\begin{array}{l}1575 / 2-37066_{3 / 2} \\
224_{3 / 2}-4370_{3 / 2}\end{array}$ & $\begin{array}{l}+.2 \\
-.1\end{array}$ & -- \\
\hline 40 & 4536.014 & 22039.60 & $(.512, .713)$ & $217_{7 / 2}-4381_{7 / 2}$ & -.01 & - & 4 & 4699. 69 & 21272.0 & & $275_{9 / 2}-4878_{7 / 2}$ & .0 & -- \\
\hline & & & $\begin{array}{l}1.246,1.452 \text {, } \\
1.699 .\end{array}$ & & & & $\begin{array}{l}50 \\
60\end{array}$ & 4700.440 & 21268. 65 & & $217_{7 / 2}-43045 / 2$ & +.03 & -- \\
\hline 3 & 4537.54 & 22032.2 & & $324_{5 / 2}-5446_{3 / 2}$ & -.1 & -. & $\begin{array}{r}60 \\
2\end{array}$ & $\begin{array}{l}4705.04 \\
4711.22\end{array}$ & $\begin{array}{l}21247.8 \\
21220.0\end{array}$ & & $\begin{array}{l}221_{9 / 2}-4340_{7 / 2} \\
280_{5 / 2}-49255_{3 / 2}\end{array}$ & $\begin{array}{r}.0 \\
+.3\end{array}$ & -- \\
\hline & 4001.04 & 22002.2 & & $278_{3 / 2}-4985_{1 / 2}$ & +.3 &.- & 20 & 4712. 76 & $\begin{array}{l}21220.0 \\
21213.0\end{array}$ & & $\begin{array}{l}280_{5 / 2}-4925_{3 / 2} \\
247_{7 / 2}-4593_{7 / 2}\end{array}$ & $\begin{array}{r}+.3 \\
.0\end{array}$ & -- \\
\hline 50 & 4541.80 & 22011.5 & & $2896_{3 / 2}-5097_{5 / 2}$ & -.1 & -- & 4 & 4725.02 & 21158.0 & & $263_{13 / 2}-4750_{13 / 2}$ & +.2 & -- \\
\hline 100 & 4545.174 & 21995. 19 & (0w) $1.356 \ldots$ & $157_{5 / 2}-37 \gamma_{67 / 2}$ & -.04 & -- & 20 & 4725.93 & 21153.9 & & $231_{5 / 2}-4430_{5 / 2}$ & 0 & - \\
\hline $\begin{array}{r}10 \\
4\end{array}$ & 4545.53 & 21993. 5 & - & $224_{3 / 2}-4441_{1 / 2}$ & .0 & -- & 80 & 4727.60 & 21146.5 & & $224_{3 / 2}-43566_{5 / 2}$ & .0 & -- \\
\hline $\begin{array}{l}4 \\
3\end{array}$ & $\begin{array}{l}4551.502 \\
4556.025\end{array}$ & $\begin{array}{l}21964.61 \\
21942.80\end{array}$ & & $\begin{array}{l}306_{9 / 2}-5261_{7 / 2} \\
304_{3 / 2}-5246_{1 / 2}\end{array}$ & $\begin{array}{l}-.07 \\
-.05\end{array}$ & -- & $2 \mathrm{~h}$ & 4730.43 & 21133.8 & & $\begin{array}{l}1669 / 2-37767 / 2 \\
298 \%-5093: 2\end{array}$ & +.1 & -- \\
\hline 4 & 4557.33 & 21936.5 & (n) & $313_{9 / 2}-53337 / 2$ & .0 & -- & 4 & 4733.87 & 21118.5 & & $\begin{array}{l}298_{5 / 2}-5093_{5 / 2} \\
265_{5 / 2}-47 \gamma 7_{3 / 2}\end{array}$ & $\begin{array}{l}.0 \\
.0\end{array}$ & $-\overline{5}$ \\
\hline 50 & 4559.268 & 21927. 19 & & $157_{5 / 2}-3769_{5 / 2}$ & -.05 & - & 4 & 4743. 46 & 21075. 7 & & $319_{7 / 2}-5305_{5 / 2}$ & -.1 & $\ldots$ \\
\hline 50 & 4559.682 & 21925.20 & $(.129) .616$ & $247_{7 / 2}-46645 / 2$ & .00 & -- & 5 & $4746-24$ & 21063.4 & & $304_{3 / 2}-5159_{5 / 2}$ & -.1 & -- \\
\hline 7 & 4563.63 & 21906.2 & 874. & $333_{11 / 2}-5522_{11 / 2}$ & -.01 & -8 & $\begin{array}{r}8 \\
80\end{array}$ & $\begin{array}{l}\text { 4748. } 06 \\
4748.38\end{array}$ & 21055. 3 & & $2759 / 2-485611 / 2$ & .0 & -- \\
\hline 4 & 4564.633 & 21901. 42 & & $166_{9 / 2}-3852_{7 / 2}$ & -.04 & -- & 80 & 4748. 38 & 21053. 9 & & $\begin{array}{r}164_{3 / 2}-37388_{5 / 2} \\
142_{7 / 2}-35265 / 2\end{array}$ & $\begin{array}{r}.0 \\
-.1\end{array}$ & -- \\
\hline 50 & 4565.30 & 21898. 2 & & $151_{1 / 2}-3706_{3 / 2}$ & +.4 & 27 & 40 & 4749.03 & 21051.0 & & $197_{1 / 2}-4080_{3 / 2}$ & +.1 & - \\
\hline 60 & 4565.51 & 21897.2 & & $101 / 2-01003 / 2$ & -.6 & 28 & 5 & 4750.99 & 21042.4 & & $266_{5 / 2}-477 O_{3 / 2}$ & .0 & -. \\
\hline $2 \mathrm{~h}$ & 4568.748 & 21881.70 & 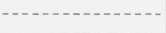 & $271_{7 / 2}-4902_{5 / 2}$ & +.06 & & 1 & 4751.23 & 21041.3 & & $261_{3 / 2}-4718_{1 / 2}$ & .0 & -- \\
\hline (1) & 4571.91 & 21866.6 &.- & $272_{9 / 2}-49037 / 2$ & +.1 & 23 & 10 & 4751.345 & 21040.78 & & $261_{3 / 2}-4717_{3 / 2}$ & +.1 & -- \\
\hline 4 & 4572.195 & 21865. 20 & $\cdots$ & $280_{5 / 2}-4989_{3 / 2}$ & -.05 & -- & 4 & 4752.10 & 21037.4 & & $244_{5 / 2}-45467 / 2$ & .0 & -- \\
\hline 2 & 4575.935 & 21847. 33 & & $\begin{array}{l}298_{5 / 2}-51647 / 2 \\
278_{3 / 2}-4966_{3 / 2}\end{array}$ & -.11 & -- & 40 & 4758.833 & 21007.67 & & $115_{5 / 2}-3259_{3 / 2}$ & .00 & -- \\
\hline $\begin{array}{l}3 \\
4\end{array}$ & $\begin{array}{l}4577.550 \\
4578.251\end{array}$ & $\begin{array}{l}21839.62 \\
21836.28\end{array}$ & & $\begin{array}{l}278_{3 / 2}-4966_{3 / 2} \\
221_{9 / 2}-4399_{11 / 2}\end{array}$ & $\begin{array}{l}+.02 \\
+.02\end{array}$ & -- & 20 & 4763.672 & 20986. 34 & & $266_{5 / 2}-4766_{7 / 2}$ & $\begin{array}{l}+.09 \\
+.06\end{array}$ & -- \\
\hline & & 21000.20 & & $314_{3 / 2}-5329_{3 / 2}$ & .0 & - & 4 & 4771.807 & $\begin{array}{l}20900.04 \\
20950.56\end{array}$ & & $\begin{array}{l}224_{3 / 2}-4.341_{5 / 2} \\
304_{3 / 2}-5147_{5 / 2}\end{array}$ & $\begin{array}{l}+.06 \\
+.01\end{array}$ & -- \\
\hline $3 \mathrm{~h}$ & 4578.93 & 21833.1 & & $324_{5 / 2}-5426_{5 / 2}$ & +.1 & -. & $4 \mathrm{~h}$ & 4778. 99 & 20919.1 & & $224_{3 / 2}-43343 / 2$ & +.1 & - \\
\hline 100 & 4580,68 & & & $280_{5 / 2}-49867 / 2$ & +.2 & -- & $2 \mathrm{~h}$ & 4781.83 & 20906.6 & -- & $244_{5 / 2}-4533_{5 / 2}$ & .0 & -- \\
\hline & 4580.68 & 21824.7 & $\begin{array}{l}(.341, .562) \\
1.177,1.394,\end{array}$ & $115_{5 / 2}-3340_{5 / 2}$ & -.1 & -- & $\begin{array}{r}10 \\
3\end{array}$ & $\begin{array}{l}4789.206 \\
4789.60\end{array}$ & $\begin{array}{l}20874.45 \\
20872.7\end{array}$ & & $\begin{array}{l}319_{7 / 2}-52857.2 \\
261_{3 / 2}-4700_{3 / 2}\end{array}$ & $\begin{aligned} & .00 \\
-.1 & \end{aligned}$ & -- \\
\hline & & & 1.611 . & & & & 200 & 4791. 418 & 20864. 81 & $(.149, .446)$ & $\begin{array}{l}261_{3 / 2}-4700_{3 / 2} \\
150_{7 / 2}-3592_{9 / 2}\end{array}$ & -.02 & - \\
\hline $\begin{array}{l}3 \\
8\end{array}$ & $\begin{array}{l}4586.06 \\
4587.141\end{array}$ & $\begin{array}{l}21799.1 \\
21793.96\end{array}$ & & $\begin{array}{l}197_{1 / 2}-4155_{3 / 2} \\
217_{7 / 2}-4356_{5 / 2}\end{array}$ & -.1 & - & & & & $1.310,1.599$, & & & \\
\hline (2) & 4588.95 & 21785.4 & & $\begin{array}{l}217_{7 / 2}-4356_{5 / 2} \\
247_{7 / 2}-4550_{5 / 2}\end{array}$ & $\begin{array}{l}.00 \\
+.2\end{array}$ & $\overline{2} \overline{3}$ & & & & $\begin{array}{l}1.887,2.178 \text {, } \\
2.468 \text {. }\end{array}$ & & & \\
\hline 3 & 4590.540 & 21777.82 & & $298_{5 / 2}-5157_{3 / 2}$ & +.15 & - & 4 & 4796.895 & 20840.99 & & $306_{9 / 2}-5148_{9 / 2}$ & +.03 & \\
\hline 20 & 4591.682 & 21772.40 & & $204_{3 / 2}-4225_{3 / 2}$ & -.06 & -. & 15 & 4799.127 & 20831. 29 & & $204_{3 / 2}-4131_{5 / 2}$ & .00 &.- \\
\hline 4 & 4592.53 & 21768. 4 & $\cdots$ & $2857 / 2-50317 / 2$ & -.2 & -- & 9 & 4799.49 & 20829.7 & & $271_{7 / 2}-4797_{5 / 2}$ & .0 & -. \\
\hline 4 & 4595.263 & 21755.44 & & $\begin{array}{l}311_{5 / 2}-52966_{3 / 2} \\
319_{7 / 2}-5373_{7 / 2}\end{array}$ & $\begin{array}{l}.0 \\
-.03\end{array}$ & -- & $\begin{array}{l}1 \\
3\end{array}$ & $\begin{array}{l}4802.28 \\
4813.139\end{array}$ & 20817. 6 & & $\begin{array}{l}314_{3 / 2}-5227_{3 / 2} \\
298_{5 / 2}-505 \gamma_{3 / 2}\end{array}$ & -.1 & -- \\
\hline 5 & 4597.32 & 21745.7 & $\ldots$ & $278_{3 / 2}-4957_{5 / 2}$ & +.2 & - & $\ddot{2}$ & 4814. 92 & 20763.0 & & $\begin{array}{l}298_{5 / 2}-5057_{3 / 2} \\
288_{7 / 2}-4957_{5 / 2}\end{array}$ & $\begin{array}{l}+.01 \\
-.2\end{array}$ & -- \\
\hline $\begin{array}{r}1 \\
1 \mathrm{~h}\end{array}$ & $\begin{array}{l}4599.764 \\
4601.13\end{array}$ & $\begin{array}{l}21734.15 \\
21727.7\end{array}$ & & $\begin{array}{l}1737 / 2-39066_{5 / 2} \\
3066_{12}-5237_{7 / 2}\end{array}$ & $\begin{array}{l}+.05 \\
-1\end{array}$ & -- & 2 & 4816.03 & 20758. 2 & & $319_{7 / 2}-527 / 49 / 2$ & -.1 & - \\
\hline & & & & & & & & & & & & & \\
\hline
\end{tabular}


Table 2. Classified lines of Re I-Continued

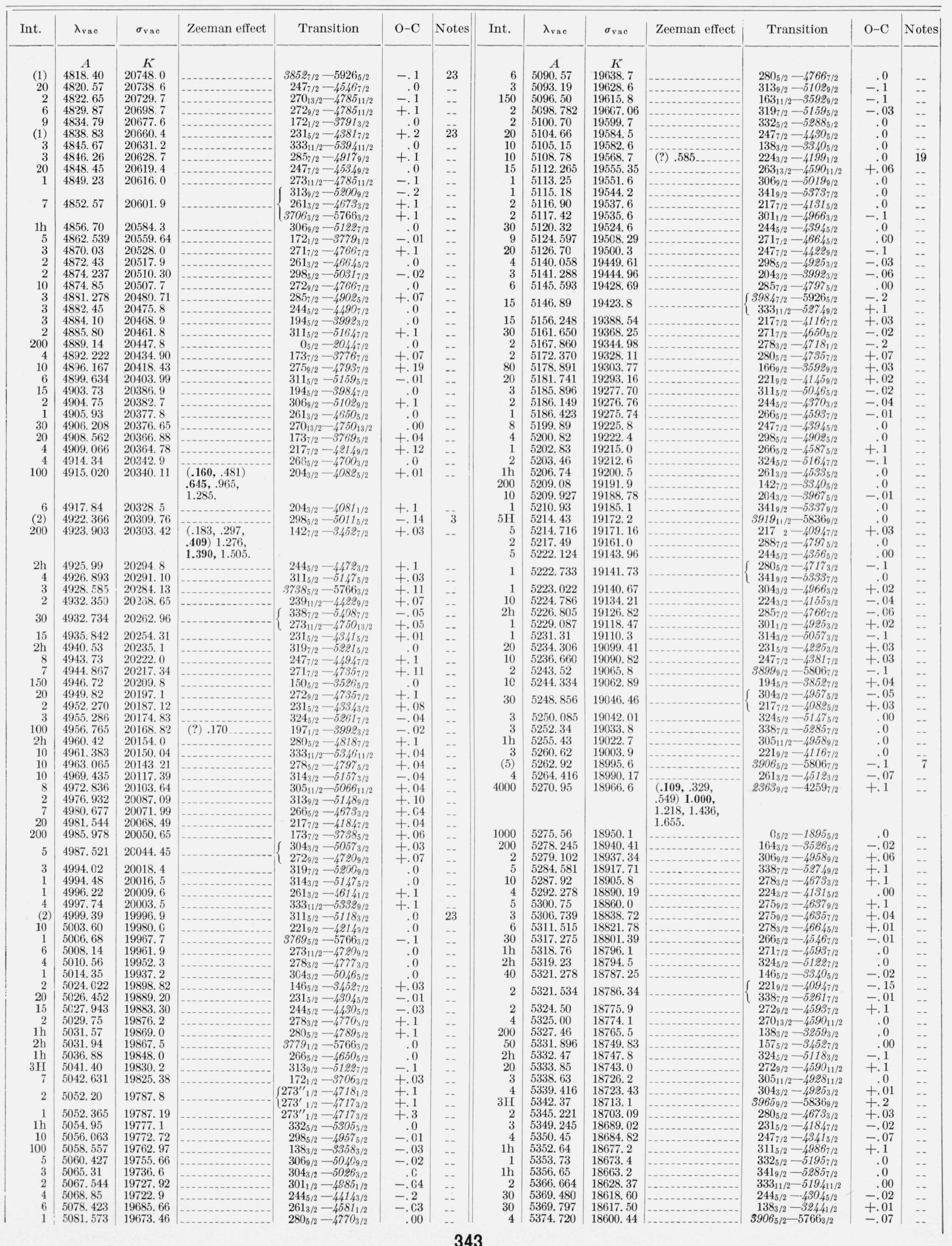


TABle 2. Classified lines of Re I-Continued

\begin{tabular}{|c|c|c|c|}
\hline Int. & $\lambda_{\mathrm{vac}}$ & $\sigma_{\mathrm{vac}}$ & Zeeman effect \\
\hline & $\underset{5377.10}{A}$ & $\begin{array}{c}K \\
18592.2\end{array}$ & \\
\hline $\begin{array}{r}200 \\
5\end{array}$ & $\begin{array}{l}5377.10 \\
5378.163\end{array}$ & $\begin{array}{l}18592.2 \\
18588.53\end{array}$ & \\
\hline 3 & 5379.707 & 18583.20 & \\
\hline $1 \mathrm{~h}$ & 5390.18 & 18547.1 & \\
\hline 9 & 5396.50 & 18525.4 & \\
\hline 1 & 5409.52 & 18480.8 & \\
\hline 2 & 5410.01 & 18479.1 & \\
\hline (1) & 5416.26 & 18457.8 & \\
\hline $5 \mathrm{H}$ & 5417.78 & 18452.6 & \\
\hline (2) & 5421.75 & 18439.1 & \\
\hline 2 & 5423.29 & 18433.9 & \\
\hline 4 & 5423.60 & 18432.8 & \\
\hline 6 & 5423.81 & 18432.1 & \\
\hline 30 & 5431.90 & 18404.6 & \\
\hline 10 & 5437.03 & 18387.3 & \\
\hline 3 & 5437.41 & 18386.0 & \\
\hline $2 \mathrm{~h}$ & 5443.31 & 18366.1 & \\
\hline 15 & 5447.92 & 18350.5 & \\
\hline $\begin{array}{r}4 \\
2 \mathrm{~h}\end{array}$ & $\begin{array}{l}5456.316 \\
5458.92\end{array}$ & $\begin{array}{l}18322.29 \\
18313.5\end{array}$ & \\
\hline 30 & 5460.644 & 18307.77 & \\
\hline 7 & 5465.43 & 18291.7 & \\
\hline 3 & 5467.54 & 18284.7 & \\
\hline 5 & 5481.003 & 18239.76 & \\
\hline 0 & & 18259.70 & \\
\hline 3 & $\begin{array}{l}5492.19 \\
5494.34\end{array}$ & 18202. 6 & \\
\hline 2 & 5494.34 & 18195.5 & \\
\hline 2 & 5495.69 & 18191.0 & \\
\hline $1 \mathrm{~h}$ & 5499. 79 & 18177.5 & \\
\hline 5 & 5501.921 & 18170.42 & \\
\hline 3 & 5505.60 & 18158.3 & \\
\hline 2 & 5514.99 & 18127.4 & \\
\hline 4 & 5519.53 & 18112.5 & \\
\hline 15 & 5521.10 & 18107.3 & \\
\hline $2 \mathrm{~h}$ & 5521.83 & 18104.9 & \\
\hline 8 & 5523.40 & 18099.8 & \\
\hline 50 & 5532.676 & 18069.41 & \\
\hline $1 \mathrm{~h}$ & 5534.35 & 18063.9 & \\
\hline $2 \mathrm{~h}$ & 5535.94 & 18058.8 & \\
\hline $2 \mathrm{~h}$ & 5545.15 & 18028.8 & \\
\hline (1h) & 5551.05 & 18009.6 & \\
\hline 7 & 5557.20 & 17989.7 & \\
\hline 100 & 5563.24 & 17970.1 & \\
\hline 7 & 5564.12 & 17967.3 & \\
\hline 30 & 5573.47 & 17937.2 & \\
\hline 8 & 5577.69 & 17923.6 & \\
\hline 3 & 5578.38 & 17921.4 & \\
\hline 2 & 5582.89 & 17906.9 & \\
\hline 30 & 5584.718 & 17901.03 & \\
\hline 2 & 5592.68 & 17875.6 & \\
\hline 6 & 5594.856 & 17868.60 & \\
\hline 3 & 5601.92 & 17846.1 & \\
\hline $2 \mathrm{~h}$ & 5604.15 & 17839.0 & \\
\hline 8 & 5607.21 & 17829.2 & \\
\hline 3 & 5607.41 & 17828.6 & \\
\hline $3 \mathrm{~h}$ & 5608.803 & 17824.16 & \\
\hline 3 & 5610.516 & 17818. 72 & \\
\hline 2 & 5614.85 & 17805.0 & \\
\hline 4 & 5619.76 & 17789.4 & \\
\hline 1 & 5621. 22 & 17784.8 & \\
\hline 8 & 5625.441 & 17771.44 & \\
\hline 3 & 5635.45 & 17739.9 & -... \\
\hline 8 & 5653.018 & 17684.75 & \\
\hline 4 & 5658.68 & 17667.1 & \\
\hline 5 & 5662.83 & 17654.1 & \\
\hline 3 & 5664.69 & 17648.3 & \\
\hline 3 & 5665.01 & 17647.3 & \\
\hline & & 19. & \\
\hline 100 & 5667.88 & 17638.4 & \\
\hline 3 & 5671.04 & 17628. 6 & \\
\hline 3 & 5678.34 & 17605.9 & \\
\hline $2 \mathrm{~h}$ & 5683.40 & 17590.2 & \\
\hline 2 & 5684. 31 & 17587.4 & \\
\hline 2 & 5686.81 & 17579.7 & \\
\hline 6 & 5689.75 & 17570.6 & \\
\hline 2 & 5692.46 & 17562. 2 & \\
\hline 30 & 5711. 426 & 17503. 90 & \\
\hline 5 & 5714.02 & 17495.9 & $-\ldots$ \\
\hline 15 & 5716.950 & 17486.99 & \\
\hline 9 & 5725.626 & 17460.49 & \\
\hline 3 & 5727.90 & 17453.6 & \\
\hline 1 & 5730.96 & 17444. 2 & \\
\hline 5 & 5733.06 & 17437.9 & \\
\hline 5 & 5734. 26 & 17434.2 & \\
\hline 2 & 5736.27 & 17428.1 & \\
\hline 2 & 5737.32 & 17424.9 & \\
\hline $3 \mathrm{H}$ & 5738.17 & 17422.3 & \\
\hline 7 & 5739.445 & 17418.45 & \\
\hline 10 & 5740.32 & 17415.8 & \\
\hline $2 \mathrm{~h}$ & 5749.86 & 17386.9 & $\cdots$ \\
\hline 200 & 5752.93 & 17377.6 & (?) $.752 \dagger \ldots$ \\
\hline 3 & 5763.41 & 17346.0 & - non \\
\hline $1 \mathrm{~h}$ & 5773.32 & 17316.3 & $\ldots$ \\
\hline 300 & 5776.83 & 17305.7 & $-1 .-1$ \\
\hline 1 & 5778. 16 & 17301.8 & \\
\hline
\end{tabular}


Table 2. Classified lines of Re I-Continued

\begin{tabular}{|c|c|c|}
\hline Int. & $\lambda_{\mathrm{vac}}$ & $\sigma_{\mathrm{vac}}$ \\
\hline 10 & $\underset{6373.47}{A}$ & $\begin{array}{c}K \\
15685.7\end{array}$ \\
\hline 15 & 6382.94 & 15662.4 \\
\hline 20 & 6405.98 & 15606.1 \\
\hline (3) & 6406.16 & \\
\hline 20 & 6411.47 & 15592.7 \\
\hline $2 \mathrm{~h}$ & 6416.13 & 15581.4 \\
\hline $2 \mathrm{~h}$ & 6422.24 & 15566.6 \\
\hline 3 & 6436.69 & 15531.6 \\
\hline 8 & 6449.28 & 15501.3 \\
\hline 5 & 6501.17 & 15377.58 \\
\hline 7 & 6502.22 & 15375.10 \\
\hline 80 & 6511.47 & 15353.27 \\
\hline 20 & 6515.25 & 15344,36 \\
\hline 7 & 6529.21 & 15311.56 \\
\hline 10 & 6544.91 & 15274.82 \\
\hline 8 & 6545.72 & 15272.93 \\
\hline 4 & 6554.66 & 15252.10 \\
\hline 10 & 6557.67 & 15245.11 \\
\hline 70 & 6577.11 & 15200.04 \\
\hline 100 & 6592.52 & 15164.51 \\
\hline 250 & 6605. 19 & 15135.43 \\
\hline 4 & 6612.53 & 15118. 62 \\
\hline 100 & 6623. 91 & 15092.65 \\
\hline 30 & 6637.25 & 15062.32 \\
\hline 200 & 6652.39 & 15028.03 \\
\hline 5 & 6658.74 & 15013.70 \\
\hline 20 & 6665. 32 & 14998.89 \\
\hline 8 & 6675.23 & 14976.51 \\
\hline 20 & 6683.23 & 14958.57 \\
\hline 7 & 6688.75 & 14946.34 \\
\hline 10 & 6711.30 & 14896.13 \\
\hline 2 & 6723. 19 & 14869.77 \\
\hline $3 \mathrm{~h}$ & 6723.55 & 14868.98 \\
\hline 6 & 6724.98 & 14865.82 \\
\hline 10 & 6761.19 & 14786.20 \\
\hline 6 & 6799. 59 & 14702.70 \\
\hline 5 & 6801.66 & 14698.23 \\
\hline 5 & 6805.35 & 14690.26 \\
\hline 4 & 6811.37 & \\
\hline 300 & 6813.41 & $\begin{array}{l}14677.28 \\
14672.88\end{array}$ \\
\hline 2 & 6816.41 & $\begin{array}{l}146 / 2.88 \\
14666.43\end{array}$ \\
\hline 2 & & 14666.43 \\
\hline 300 & 6829.90 & 14637.45 \\
\hline & & \\
\hline $3 \mathrm{H}_{5}$ & $\begin{array}{l}6835.35 \\
6844.06\end{array}$ & $\begin{array}{l}14625.78 \\
14607.17\end{array}$ \\
\hline 5 & 6844,44 & $\begin{array}{l}14607.17 \\
14606.36\end{array}$ \\
\hline 8 & 6874.35 & $\begin{array}{l}14606.36 \\
14542.81\end{array}$ \\
\hline 3 & 6882.11 & $\begin{array}{l}14542.81 \\
14526.41\end{array}$ \\
\hline 3 & 6963.50 & 14356.63 \\
\hline 8 & 6967.67 & 14348.04 \\
\hline 200 & 6971.53 & 14340.10 \\
\hline 2 & 6976.67 & 14329.54 \\
\hline 20 & 6985. 20 & 14312. 02 \\
\hline 150 & 7006.63 & 14268. 26 \\
\hline 5 & 7012.56 & 14256.20 \\
\hline 200 & 7024.15 & 14232. 66 \\
\hline (2) & 7027.14 & 14226.61 \\
\hline 7 & 7028.47 & 14223. 92 \\
\hline 10 & 7058.24 & 14163.92 \\
\hline 6 & 7059. 98 & 14160.43 \\
\hline 3 & 7065.39 & 14149.59 \\
\hline 10 & 7066.47 & 14147.43 \\
\hline (10) & 7129.25 & 14022.85 \\
\hline (1) & 7139.64 & 14002.45 \\
\hline 10 & 7172.20 & 13938.87 \\
\hline 4 & 7198.25 & 13888. 44 \\
\hline 20 & 7228.03 & 13831. 22 \\
\hline 3 & 7233.90 & 13820.00 \\
\hline 41 & 7237.26 & 13813.56 \\
\hline 150 & 7246.49 & 13795.97 \\
\hline 100 & 7246.80 & 13795. 38 \\
\hline 4 & 7263.86 & 13762.99 \\
\hline 150 & 7273.84 & 13744. 11 \\
\hline 300 & 7292.72 & 13708.52 \\
\hline 5 & 7307.55 & 13680.71 \\
\hline (4) & 7316.1 & 13664.7 \\
\hline (2) & 7318. 02 & 13661.13 \\
\hline 7 & 7324. 20 & 13649.60 \\
\hline (1) & 7346.88 & 13607. 47 \\
\hline 50 & 7352.04 & 13597.91 \\
\hline (2) & 7362.12 & 13579.30 \\
\hline 3 & 7382.67 & 13541.51 \\
\hline 1 & 7384.07 & 13538. 94 \\
\hline 20 & 7386.35 & 13534. 74 \\
\hline 4 & 7390.71 & 13526. 76 \\
\hline 2 & 7396.04 & 13517.02 \\
\hline $3 \mathrm{~h}$ & 7404.28 & 13501.98 \\
\hline & & \\
\hline & 7409.46 & $\begin{array}{l}13492.54 \\
13485.35\end{array}$ \\
\hline (2) & 7413.41 & 13485. 35 \\
\hline 3 & 7416.44 & $\begin{array}{l}13479.85 \\
13451.74\end{array}$ \\
\hline 2 & 7431.93 & 13451.74 \\
\hline 2 & 7437. 79 & 13441. 14 \\
\hline 1 & 7442.75 & 13432.19 \\
\hline (1) & 7446.97 & 13424.58 \\
\hline
\end{tabular}


TABLE 2. Classified lines of Re I-Continued

\begin{tabular}{|c|c|c|c|c|c|c|c|c|c|c|c|c|c|}
\hline Int. & $\lambda_{\mathrm{vac}}$ & $\sigma_{\mathrm{vac}}$ & Zeeman effect & Transition & $\mathrm{O}-\mathrm{C}$ & Notes & Int. & $\lambda_{\mathrm{vac}}$ & $\sigma_{\mathrm{Vac}}$ & Zeeman effect & Transition & $\mathrm{O}-\mathrm{C}$ & Notes \\
\hline 1 & $\begin{array}{c}A \\
8686.94\end{array}$ & $\begin{array}{c}K \\
11508.36\end{array}$ & & $338-18-1588_{-1}$ & & & & $\underset{974966}{A}$ & $K^{K}$ & & & & \\
\hline 20 & 8697.25 & 11494. 72 & & $\begin{array}{l}338_{7 / 2}-4583_{5 / 2} \\
3069 / 2-42149 / 2\end{array}$ & $\begin{array}{l}-.01 \\
-.01\end{array}$ & -- & $\begin{array}{l}6 \\
4\end{array}$ & $\begin{array}{l}9749.66 \\
9752.08\end{array}$ & $\begin{array}{l}10253.95 \\
10251.41\end{array}$ & & $\begin{array}{l}231_{5 / 2}-3340_{5 / 2} \\
275_{9 / 2}-3776_{7 / 2}\end{array}$ & $\begin{array}{l}+.03 \\
+.07\end{array}$ & -- \\
\hline (2) & 8715.7 & 11470.4 & & $319_{7 / 2}-4345_{9 / 2}$ & +.1 & 31 & 20 & 9762.65 & 10240.30 & & $271_{7 / 2}-3738_{5 / 2}$ & +.02 & -- \\
\hline (6) & 8721.64 & 11462.59 & & $3259_{3 / 2}-4405_{5 / 2}$ & +.01 & 31 & 9 & 9831.35 & 10168. 75 & & $224_{3 / 2}-3259_{3 / 2}$ & -.05 & -- \\
\hline $3 \mathrm{~h}$ & 8750.44 & 11424.86 & & $319_{7 / 2}-4340_{7 / 2}$ & -.02 & -- & 20 & 9842.65 & 10157. 07 & & $319_{7 / 2}-42140 / 2$ & .00 & -- \\
\hline 30 & 8797.70 & 11363.48 & & $298_{5 / 2}-4116_{7 / 2}$ & -.05 & -. & $15 \mathrm{sd}$ & 9872.38 & 10126. 48 & & $298_{5 / 2}-3992_{3 / 2}$ & +.11 & -. \\
\hline 3 & 8845.23 & 11302.42 & & $285_{7 / 2}-39847 / 2$ & -.20 & -. & 1 & 9901.72 & 10096. 48 & & $314_{3 / 2}-4155_{3 / 2}$ & +.02 & -- \\
\hline 3 & 8851.32 & 11294.64 & & $3940_{5 / 2}-4470_{5 / 2}$ & .00 & - & 5 & 9903.30 & 10094.87 & & $244_{5 / 2}-3452_{7 / 2}$ & +.02 & -. \\
\hline 1 & 8871.82 & 11268.54 & & $341_{9 / 2}-4546_{7 / 2}$ & -.02 & - & 4 & 9908.97 & 10089.09 & & $40947 / 2-5104_{5 / 2}$ & +.06 & - \\
\hline 15 & 8882.95 & 11254.43 & & $266_{5 / 2}-8791_{3 / 2}$ & -.01 & -- & 1 & 9937.8 & 10059.9 & & $332_{5 / 2}-43343 / 2$ & -.3 & $\ldots$ \\
\hline 15 & 8886.58 & 11249.83 & & $261_{5 / 2}-3738_{5 / 2}$ & -.01 & .. & 20 & 9943.70 & 10053.85 & & $313_{9 / 2}-41459 / 2$ & -.03 & -- \\
\hline 5 & 8927. 28 & 11198.54 & & $3069 / 2-41847 / 2$ & +.02 & -- & 200 & 9949.90 & 10047. 59 & & $40947 / 2-50999 / 2$ & -.03 & -- \\
\hline 7 & 8966.63 & 11149.40 & & $\left\{39847 / 2-5099_{9 / 2}\right.$ & .00 & -. & 3 & 9953.02 & 10044.44 & & $298_{5 / 2}-39847 / 2$ & +.07 & -- \\
\hline 4 & 8969.21 & 11146.19 & & $\left(341_{9 / 2}-45349 / 2\right.$ & +.10 & -- & 60 & 9955.45 & 10041.99 & & $40947 / 2-50987 / 2$ & +.05 & -- \\
\hline & 8971.14 & $\begin{array}{l}11140.19 \\
11143.79\end{array}$ & & $298_{5 / 2}-40947 / 2$ & +.04 & -- & 2 & 9964.90 & 10032.47 & & $4405_{5 / 2}-5408_{7 / 2}$ & +.01 & -. \\
\hline 2 & & 11170.78 & & $39847 / 2-5098_{7 / 2}$ & +.09 & -. & 1 & 9970.87 & 10026.46 & & $40947 / 2-5097_{5 / 2}$ & -.18 & -- \\
\hline 1 & 8983.55 & 11128.39 & & $\left\{39847 / 2-5097_{5 / 2}\right.$ & -.03 & -- & 4 & 9976.45 & 10020.85 & & $224_{3 / 2}-32441 / 2$ & +.07 & -- \\
\hline 2 & 8988.40 & 11122.39 & & $285_{7 / 2}-3967_{5 / 2}$ & .00 & -.- & 7 & 10019. 21 & 9978. 09 & & $311_{5 / 2}-41167 / 2$ & +.26 & -- \\
\hline & & & & $338_{7 / 2}-44947 / 2$ & -.07 & -- & 5 & 10026. 96 & 9970.38 & & $278_{3 / 2}-3 \gamma \gamma 9_{1 / 2}$ & $\begin{array}{r}+.08 \\
+.03\end{array}$ & -- \\
\hline 1 & 8995.26 & 11113.91 & & $\left\{\begin{array}{r}2857 / 2-39659 / 2 \\
3992^{2}-5103 / 2 / 2\end{array}\right.$ & $\begin{array}{l}+.05 \\
+.08\end{array}$ & -. & $\begin{array}{l}2 \\
1\end{array}$ & $\begin{array}{l}\text { 10064. } 02 \\
10127.21\end{array}$ & 9933. 66 & & $305_{11 / 2}-4049_{13 / 2}$ & $\begin{array}{l}+.23 \\
+03\end{array}$ & - \\
\hline 1 & 9003.24 & 11104.06 & & $\begin{array}{r}399 Z_{3 / 2}-370 \sigma_{3 / 2} \\
266_{5 / 2}-376_{5 / 2}\end{array}$ & -.16 & $\ldots$ & 15 & 10128. 78 & $\begin{array}{l}9871.08 \\
9870.15\end{array}$ & & $\begin{array}{r}41107 / 2-51045 / 2 \\
298_{5 / 2}-3967_{5 / 2}\end{array}$ & $\begin{array}{l}+.00 \\
+.01\end{array}$ & -- \\
\hline 1 & 9018.9 & 11084. 75 & & $4818_{7 / 2}-5926_{5 / 2}$ & +.16 & -. & 2 & 10138. 24 & 9860.94 & & $319_{7 / 2}-41847 / 2$ & +.08 & - \\
\hline 3 & 9032.46 & 11068.14 & & $311_{5 / 2}-4225_{3 / 2}$ & +.03 & - & $\overline{1}$ & 10146.84 & 9852.59 & & $314_{3 / 2}-4131_{5 / 2}$ & +.19 & -. \\
\hline 3 & 9058.53 & 11036. 28 & & $266_{5 / 2}-3769_{5 / 2}$ & +.05 & -. & 100 & 10169.85 & 9830.29 & & $41167 / 2-5099_{9 / 2}$ & +.05 & -- \\
\hline 4 & 9059.85 & 11034. 67 & & $280_{5 / 2}-3906_{5 / 2}$ & +.07 & -. & 20 & 10175. 68 & 9824.66 & & $4116_{7 / 2}-50987 / 2$ & +.10 & -- \\
\hline 5 & 9070.74 & 11021.43 & & $298_{5 / 2}-4082_{5 / 2}$ & -.02 & -- & 1 & 10191.57 & 9809.35 & & $4116_{7 / 2}-5097_{5 / 2}$ & +.09 & -- \\
\hline 4 & 9081.45 & 11008.43 & & $298_{5 / 2}-4080_{3 / 2}$ & -.04 & .- & 2 & 10201.86 & 9799.44 & & $4856_{11 / 2}-58369 / 2$ & +.17 & -- \\
\hline 2 & 9083.03 & 11006.52 & & $2759 / 2-38527 / 2$ & +.09 & -- & 20 & 10206.32 & 9795.16 & & $301_{1 / 2}-3992_{3 / 2}$ & -.02 & -- \\
\hline 6 & 9144.86 & 10932.10 & & $261_{5 / 2}-37063 / 2$ & +.02 & -. & 4 & 10238. 26 & 9764.60 & & $313_{9 / 2}-4116_{7 / 2}$ & -.01 & -- \\
\hline 2 & 9205.12 & 10860.53 & & $4673_{3 / 2}-5766_{3 / 2}$ & +.05 & .. & 1 & 10242. 68 & 9760.39 & & $311_{5 / 2}-40947 / 2$ & -.06 & -. \\
\hline 1 & 9220.40 & $\begin{array}{l}10800.05 \\
10842.54\end{array}$ & & $288_{7 / 2}-3967_{5 / 2}$ & -.12 & - & 5 & 10282.46 & 9722.63 & & $4131_{5 / 2}-5104_{5 / 2}$ & +.09 & -- \\
\hline 10 & 9250.02 & 10807.81 & & $244_{5 / 2}-35266_{5 / 2}$ & .00 & -. & 4 & 10287.58 & 9717.80 & & $4131_{5 / 2}-5103_{3 / 2}$ & +.09 & -- \\
\hline 4 & 9262.28 & 10793.51 & & $\begin{array}{l}3069 / 2-41459 / 2 \\
3143 / 2-42255_{3}\end{array}$ & -.04 & -. & 10 & 10332.55 & 9675.50 & & $4131_{5 / 2}-5098_{7 / 2}$ & +.05 & -- \\
\hline 15 & 9268.46 & 10786.32 & & $\begin{array}{l}314_{3 / 2}-4225_{3 / 2} \\
304_{3 / 2}-4131_{5 / 2}\end{array}$ & -.06 & -- & 1 & 10341.3 & 9667.4 & & $280_{5 / 2}-3769_{5 / 2}$ & +.1 & -- \\
\hline 1 & 9298.07 & 10751.97 & & $\begin{array}{l}304_{3 / 2}-4181_{5 / 2} \\
341_{9 / 2}-44947 / 2\end{array}$ & -.10 & - & $\begin{array}{r}4 \\
10\end{array}$ & 10349.0 & 9660.16 & & $4131_{5 / 2}-5097_{5 / 2}$ & +.01 & -- \\
\hline 1 & 9307.72 & 10740.82 & & $\begin{array}{l}341_{9 / 2}-449477 / 2 \\
3139 / 2-42149 / 2\end{array}$ & +.12 & $\cdots$ & $\begin{array}{l}10 \\
20\end{array}$ & 10464. 25 & 9553. 73 & & $278_{3 / 2}-3738_{5 / 2}$ & -.03 & -- \\
\hline 3 & 9325.90 & 10719.88 & & $\begin{array}{l}313_{9 / 2}-42149 / 2 \\
266_{5 / 2}-3738_{5 / 2}\end{array}$ & +.06 & $\begin{array}{l}-- \\
-\end{array}$ & $\begin{array}{r}20 \\
2\end{array}$ & 10556.54 & 9470.21 & & $319_{7 / 2}-4145_{9 / 2}$ & +.02 & -. \\
\hline 20 & 9363.13 & 10677.25 & & $\begin{array}{l}266_{5 / 2}-37388_{5 / 2} \\
301_{1 / 2}-4080_{3 / 2}\end{array}$ & -.10 & $\cdots$ & $\begin{array}{l}2 \\
8\end{array}$ & 10593. 74 & 9436.95 & & $231_{5 / 2}-3259_{3 / 2}$ & +.13 & -- \\
\hline 2 & 9370.87 & 10668.44 & & $\begin{array}{l}301_{1 / 2}-4080_{3 / 2} \\
332_{5 / 2}-43945 / 2\end{array}$ & -.03 & & $\begin{array}{r}8 \\
40\end{array}$ & 19599. 31 & 9432.00 & & $194_{5 / 2}-28887 / 2$ & +.17 & -- \\
\hline 10 & 9380.24 & 10657.78 & & $\begin{array}{l}332_{5 / 2}-43945 / 2 \\
311_{5 / 2}-41847 / 2\end{array}$ & +.11 & $\cdots$ & $\begin{array}{r}40 \\
8\end{array}$ & 10618. 45 & 9414.99 & & $142_{7 / 2}-23639 / 2$ & +.03 & -- \\
\hline 2 & 9391.12 & 10645.43 & & $\begin{array}{r}311_{5 / 2}-41847 / 2 \\
3940-44055 / 2\end{array}$ & +.01 & -- & 8 & 10625.57 & 9408.68 & & $324_{5 / 2}-41847 / 2$ & -.03 & -- \\
\hline 15 & 9423.45 & 10608.90 & & $\begin{array}{r}3340_{5 / 2}-4405_{5 / 2} \\
3245 / 2-43045 / 2\end{array}$ & -.05 & $-\cdot$ & $\begin{array}{r}100 \\
10\end{array}$ & 10639. 45 & 9396.40 & & $194_{5 / 2}-2885_{5 / 2}$ & +.11 & -- \\
\hline 3 & 9427.53 & 10604. 31 & $-\ldots$ & $\begin{array}{l}324_{5 / 2}-43045 / 2 \\
272_{9 / 2}-3776_{7 / 2}\end{array}$ & +.02 & -- & $\begin{array}{l}10 \\
10\end{array}$ & 10824. 22 & 9236.01 & & $278_{3 / 2}-3706_{3 / 2}$ & +.01 & -- \\
\hline 30 & 9470.15 & 10556.59 & & $\begin{array}{l}272_{9 / 2}-37766_{7 / 2} \\
271_{7 / 2}-37695 / 2\end{array}$ & $\begin{array}{l}+.01 \\
+.06\end{array}$ & -- & $\begin{array}{r}10 \\
1\end{array}$ & 10862.25 & 9203.67 & & $197_{1 / 2}-28966_{3 / 2}$ & +.03 & -- \\
\hline 1 & 9481.6 & 10543.85 & ..... & $\begin{array}{l}271_{7 / 2}-37699_{5 / 2} \\
247_{7 / 2}-3526_{5 / 2}\end{array}$ & $\begin{array}{l}+.06 \\
+.13\end{array}$ & -- & $\begin{array}{l}1 \\
1\end{array}$ & 10909.5 & 9163.8 & & $244_{5 / 2}-3358_{3 / 2}$ & +.1 & -. \\
\hline 8 & 9500.49 & 10522.88 & & $\begin{array}{l}247_{7 / 2}-35266_{5 / 2} \\
285_{7 / 2}-3906_{5 / 2}\end{array}$ & $\begin{array}{l}+.13 \\
+.09\end{array}$ & $\cdots$ & $\begin{array}{l}1 \\
2\end{array}$ & 10919. 30 & $\begin{array}{l}9155.59 \\
9143.74\end{array}$ & & $285_{7 / 2}-3769_{5 / 2}$ & +.06 & -- \\
\hline 3 & 9504.34 & 10518.62 & (n) & $\begin{array}{l}285_{7 / 2}-39065 / 2 \\
3069 / 2-4116_{7 / 2}\end{array}$ & $\begin{array}{l}+.09 \\
+.04\end{array}$ & - & $\begin{array}{l}2 \\
3\end{array}$ & 10933. 44 & $\begin{array}{l}9143.74 \\
9136.40\end{array}$ & & $304_{3 / 2}-3967_{5 / 2}$ & -.18 & -- \\
\hline 6 & 9571.75 & 10444.54 & & $\begin{array}{l}3069 / 2-41167 / 2 \\
3139 / 2-41847 / 2\end{array}$ & $\begin{array}{l}+.04 \\
-.01\end{array}$ & & 3 & 10942.23 & 9136.40 & & $261_{5 / 2}-3526_{5 / 2}$ & +.03 & -- \\
\hline 8 & 9581.12 & 10434. 32 & & & $\begin{array}{l}-.01 \\
+.01\end{array}$ & $\ldots$ & 2 & 11065.02 & 9035.02 & & $\left\{4470_{5 / 2}-53737 / 2\right.$ & -.07 & -- \\
\hline 1 & 9600.98 & 10412.75 & & $\begin{array}{r}251_{5 / 2}-3.3583 / 2 \\
273^{\prime \prime}{ }_{1 / 2}-3779_{1 / 2}\end{array}$ & $\begin{array}{r}\top .01 \\
.00\end{array}$ & -. & 1 & 11141.90 & 8972.67 & & $\begin{array}{r}15035_{7 / 2}-5939_{9 / 2} \\
332_{5 / 2}-4225_{3 / 2}\end{array}$ & $\begin{array}{l}-.04 \\
+.13\end{array}$ & - \\
\hline 3 & 9704.94 & 10301. 20 & & $3069 / 2-40947 / 2$ & .00 & -. & 4 & 11309.97 & 8839.33 & & $285_{7 / 2}-3738_{5 / 2}$ & $\begin{array}{l}+.10 \\
+.05\end{array}$ & - \\
\hline 50 & 9710.52 & 10295. 28 & & $304_{3 / 2}-4082_{5 / 2}$ & +.05 & $\cdots$ & 8 & 11383. 70 & $878 \% .08$ & & $271_{7 / 2}-3592_{9 / 2}$ & $\begin{array}{r}19 \\
+.19\end{array}$ & - \\
\hline 4 & 9722.76 & 10282.32 & - & $304_{3 / 2}-4080_{3 / 2}$ & +.07 & -- & 3 & 11453.12 & 8728.85 & & $324_{5 / 2}-4116_{7 / 2}$ & +.08 & -- \\
\hline 3 & 9748.60 & 10255.07 & - & $288_{7 / 2}-3906_{5 / 2}$ & +.02 & -- & 1 & 11615.8 & 8606.6 & & $266_{5 / 2}-3526_{5 / 2}$ & +.1 & -- \\
\hline
\end{tabular}

Notes

1. Wavelength and intensity taken from R. Velasco, Anales real soc. espan. fis. y quim. Madrid 48, 55 (1952).

2. From here (unless stated otherwise), all wavelengths and intensities have been taken from W. F. Meggers, J. Research NBS 49,187 (1952) RP2355; the intensities apply to the are observations, and all details concerning line complexity due to hyperfine structure have been omitted

3. Wavelength and intensity taken from MIT Wavelength Tables.

4. Observed only in spark by Meggers, but according to the MIT Wavelength Tables, the line belongs to the are spectrum. The intensity given applies to the spark.

5. Enhanced in spark.

6. The indicated self-reversal is an apparent one. The analysis shows that

actually a close doublet is formed by two different transitions.
7. Intensity from spark spectrum; not observed in the arc.

8. The wavelength given in the MIT Wavelength Tables fits appreciably better to the combination given (departure $0.3 \mathrm{~K}$ ); the observed $\sigma$-component is in for.

9. Observed Zeeman effect in agreement only with second transition.

10. Observed $\pi$-pattern in disagreement with classification.

11. Observed $\sigma$-pattern in disagreement with classification.

12. $\sigma$-pattern perturbed.

13. Wavelength and intensity taken from sfark observations made at the Zeeman Laboratory.

14. Probably there is a second line at this place.

15 . The $\pi$-pattern and the strongest $\sigma$-component observed are in disagreement with classification.

16. Observed Zeeman effect in agreement with both transitions.
17. Paschen-Back interaction between levels 4080 and 4081 . 18. Paschen-Back interaction between levels 5034 and 5035 .

19. Observed Zeeman effect not consistent with classification.

20. Zeeman effect suggests that levels $273^{\prime}$ and $273^{\prime \prime}$ are hyperfine components of the same level.

21. Paschen-Back interaction between levels 5103 and 5104

22. Observed Zeeman effect in agreement only with first transition.

23. Wavelength and intensity taken from W. F. Meggers, BS J. Research 6, 1027 (1931) RP322

24. In the MIT Wavelength Tables only one line is recorded with a wavelength intermediate between the two wavelengths given in Meggers' 1952 paper. The doubling is probably caused by hyperfine structure.

25. $\pi$-pattern in agreement with first combination. For the second combinaion no $g$-values are known.

26. Paschen-Back effect.

27. Apparent doubling caused by hyperfine structure; comp. note 24 .

28. In the MIT Wavelength Tables, only one line is recorded here. The doubling is a common feature of the combinations of $44700_{5,2}\left(=\left(5 d^{5} 6 s 7 s\right)^{6 \mathrm{~S}_{5,2}}\right)$; see text.

29. Wavelength taken from Meggers' 1931 paper. The MIT Wavelength Tables also give a single line, whereas in Meggers' 1952 table two lines (with intensities 150 and 100 ) have been recorded which are situated at approximately equal distances from the calculated position. The doubling may be due to hyper-

30. Wavelength and intensity taken from the MIT Wavelength Tables. Meg. gers' 1931 table gives a single line also, whereas in his 1952 table a doublet is recorded, just as for $6605 \mathrm{~A}$ (see note 29 ).

31. W avelength and intensity taken from W. F. Meggers, BS J. Research 10 757 (1933) RP564. 
TABLE 3. Unclassified Re I lines of intensity 50 or more

\begin{tabular}{|c|c|c|c|}
\hline Intensity & Wavelength & Intensity & Wavelength \\
\hline $\begin{array}{c}50 \mathrm{r} \\
100 \mathrm{R} \\
50 \\
50 \\
50\end{array}$ & \begin{tabular}{l}
\multicolumn{1}{c}{$A$} \\
2077.280 \\
2109.22 \\
2187.77 \\
2196.72 \\
2205.15
\end{tabular} & $\begin{array}{c}100 \mathrm{R} \\
50 \mathrm{r} \\
100 \mathrm{r} \\
50 \\
100 \mathrm{r}\end{array}$ & \begin{tabular}{l}
\multicolumn{1}{c}{$A$} \\
2344.776 \\
2365.316 \\
2388.569 \\
2397.31 \\
2404.340
\end{tabular} \\
\hline $\begin{array}{c}300 \mathrm{r} \\
50 \\
50 \\
50 \\
50\end{array}$ & $\begin{array}{l}2214.58 \\
2226.87 \\
2242.66 \\
2249.51 \\
2253.94\end{array}$ & $\begin{array}{c}60 \mathrm{r} \\
50 \mathrm{r} \\
100 \\
50 \mathrm{R} \\
60\end{array}$ & $\begin{array}{l}2406.695 \\
2431.538 \\
2444.09 \\
2611.536 \\
2722.206\end{array}$ \\
\hline $\begin{array}{l}50 \\
50 \\
50\end{array}$ & $\begin{array}{l}2271.04 \\
2285.30 \\
2290.715\end{array}$ & $\begin{array}{r}50 \\
50 \\
100\end{array}$ & $\begin{array}{l}2982.188 \\
4251.35 \\
6751.22\end{array}$ \\
\hline
\end{tabular}

TABLE 4. Electron configurations and spectral terms of Re I

\begin{tabular}{|c|c|c|c|c|c|c|c|c|c|c|c|}
\hline Config. & Desig. & $J$ & Level & Interval & Obs. $g$ & Config. & Desig. & $J$ & Level & Interval & Obs. $g$ \\
\hline $5 d^{5} 6 s^{2}$ & $a^{6} \mathrm{~S}$ & $21 / 2$ & 0.00 & & 1. 950 & $5 d^{4} 6 s^{2}\left(a^{5} \mathrm{D}\right) 6 p$ & $z^{6} \mathrm{D}^{\circ}$ & $01 / 2$ & 32443.61 & & \\
\hline $5 d^{5} 6 s^{2}$ & $a{ }^{4} \mathrm{P}$ & $\begin{array}{l}21 / 2 \\
11 / 2 \\
01 / 2 \\
41\end{array}$ & $\begin{array}{l}11583.96 \\
13826.12 \\
15165.89\end{array}$ & $\begin{array}{l}-2242.16 \\
-1339.77\end{array}$ & $\begin{array}{l}1.278 \\
1.485 \\
2.368\end{array}$ & & & $\begin{array}{l}11 / 2 \\
21 / 2 \\
31 / 2 \\
41 / 2\end{array}$ & $\begin{array}{l}32591.63 \\
33408.73 \\
34520.25 \\
35923.02\end{array}$ & $\begin{array}{r}148.02 \\
817.10 \\
1111.52 \\
1402.77\end{array}$ & $\begin{array}{l}1.762 \\
1.500 \\
1.454 \\
1.440\end{array}$ \\
\hline $5 d^{6}\left({ }^{5} \mathrm{D}\right) 6 s$ & $a{ }^{\circ} \mathrm{D}$ & $\begin{array}{l}41 / 2 \\
31 / 2 \\
21 / 2 \\
11 / 2 \\
01 / 2\end{array}$ & $\begin{array}{l}11754.52 \\
14216.86 \\
15770.42 \\
16327.51 \\
17238.30\end{array}$ & $\begin{array}{r}-2462.34 \\
-1553.56 \\
-557.09 \\
-910.79\end{array}$ & $\begin{array}{l}1.545 \\
1.567 \\
1.309 \\
1.706 \\
2.521\end{array}$ & $5 d^{5}\left({ }^{3} \mathrm{G}\right) 6 s$ & $b^{2} \mathrm{G}^{0}$ & $\begin{array}{l}21 / 2 \\
31 / 2 \\
4^{1 / 2} \\
51 / 2\end{array}$ & $\begin{array}{l}\text { 33281. } 65 \\
33823.66 \\
34194.27 \\
33317.57\end{array}$ & $\begin{array}{r}542.01 \\
370.61 \\
-876.70\end{array}$ & \\
\hline $5 d^{5} 6 s^{2}$ & $a^{4} \mathrm{G}$ & $\begin{array}{l}21 / 2 \\
31 / 2 \\
41 / 2 \\
51 / 2\end{array}$ & $\begin{array}{l}14621.46 \\
15058.19 \\
16619.28 \\
16307.15\end{array}$ & $\begin{array}{r}436.73 \\
1561.09 \\
-312.13\end{array}$ & $\begin{array}{l}1.151 \\
1.153 \\
1.175 \\
1.242\end{array}$ & $5 d^{4} 6 s^{2}\left({ }^{5} \mathrm{D}\right) 6 p$ & $y{ }^{6} \mathrm{P}^{\circ}$ & $\begin{array}{l}11 / 2 \\
21 / 2 \\
31 / 2 \\
21 /\end{array}$ & 33589.12 & & 2.335 \\
\hline $5 d^{5} 6 s^{2}$ & $a^{4} \mathrm{D}$ & $\begin{array}{l}51 / 2 \\
31 / 2 \\
21 / 2\end{array}$ & $\begin{array}{l}16307.15 \\
17330.82 \\
19457.89\end{array}$ & $\begin{array}{l}-2127.07 \\
-1023.84\end{array}$ & $\begin{array}{l}\text { 1. } 242 \\
\text { 1. } 255 \\
\text { 1. } 361\end{array}$ & $5 d^{5} 6 s\left(a{ }^{5} \mathrm{~S}\right) 6 p$ & $z^{4} \mathrm{P}^{\circ}$ & $\begin{array}{l}21 / 2 \\
11 / 2 \\
01 / 2\end{array}$ & $\begin{array}{l}35267.94 \\
37915.87 \\
37797.95\end{array}$ & $\begin{array}{r}-2647.93 \\
117.92\end{array}$ & $\begin{array}{l}1.385 \\
1.495 \\
2.620\end{array}$ \\
\hline & & $\begin{array}{l}11 / 2 \\
01 / 2\end{array}$ & $\begin{array}{l}20481.73 \\
19757.91\end{array}$ & $\begin{array}{r}-1023.84 \\
723.82\end{array}$ & $\begin{array}{l}1.451 \\
0.983\end{array}$ & $5 d^{5} 6 s\left(a^{5} \mathrm{G}\right) 6 p$ & $z^{6} \mathrm{G}^{\circ}$ & $\begin{array}{l}11 / 2 \\
21 / 2\end{array}$ & $\begin{array}{l}37063.65 \\
37381.41\end{array}$ & $\begin{array}{r}317.76 \\
1139.33\end{array}$ & $\begin{array}{r}0.626 \\
.990\end{array}$ \\
\hline $5 d^{5} 6 s\left(a^{7} \mathrm{~S}\right) 6 p$ & $z^{8} \mathrm{P}^{\circ}$ & $\begin{array}{l}21 / 2 \\
31 / 2 \\
41 / 2\end{array}$ & $\begin{array}{l}18950.1 \\
20447.8 \\
23631.82\end{array}$ & $\begin{array}{l}1497.7 \\
3184.0\end{array}$ & $\begin{array}{l}2.274 \\
1.926 \\
1.768\end{array}$ & & & $\begin{array}{l}31 / 2 \\
41 / 2 \\
51 / 2 \\
61 / 2\end{array}$ & $\begin{array}{l}38520.74 \\
38994.73 \\
39916.39 \\
40493.34\end{array}$ & $\begin{array}{r}1139.33 \\
473.99 \\
921.66 \\
576.95\end{array}$ & $\begin{array}{l}1.216 \\
1.25 \\
1.314 \\
1.364\end{array}$ \\
\hline $5 d^{5} 6 s^{2}$ & $a^{2} \mathrm{~F}$ & $\begin{array}{l}31 / 2 \\
21 / 2\end{array}$ & $\begin{array}{l}21775.40 \\
24425.40\end{array}$ & -2650.00 & $\begin{array}{l}\text { 1. } 135 \\
1.067\end{array}$ & $5 d^{4} 6 s^{2}\left(a^{5} \mathrm{D}\right) 6 p$ & $z^{6} \mathrm{~F}^{\circ}$ & $\begin{array}{l}01 / 2 \\
11 / 2\end{array}$ & $\begin{array}{l}37381.41 \\
39926.75\end{array}$ & 2545.34 & 0.745 \\
\hline $5 d^{5} 6 s^{2}$ & $a^{2} \mathrm{G}$ & $\begin{array}{l}41 / 2 \\
31 / 2\end{array}$ & $\begin{array}{l}22160.04 \\
24724.22\end{array}$ & -2564.18 & $\begin{array}{l}1.198 \\
1.03\end{array}$ & & & $\begin{array}{l}11 / 2 \\
31 / 2\end{array}$ & $\begin{array}{l}39064.92 \\
37765.65\end{array}$ & $\begin{array}{r}-861.83 \\
-1299.27 \\
1890.34\end{array}$ & $\begin{array}{l}\text { 1. } 335 \\
\text { 1. } 335\end{array}$ \\
\hline $5 d^{5} 6 s^{2}$ & $a^{2} \mathrm{D}$ & $\begin{array}{l}11 / 2 \\
21 / 2\end{array}$ & $\begin{array}{l}22422.83 \\
23154.81\end{array}$ & 731.98 & $\begin{array}{l}0.781 \\
1.189\end{array}$ & & & $\begin{array}{l}41 / 2 \\
5 ! / 2\end{array}$ & $\begin{array}{l}\$ 9655.99 \\
39196.7_{4}\end{array}$ & $\begin{array}{r}1890.34 \\
-459.25\end{array}$ & $\begin{array}{l}1.444 \\
1.405\end{array}$ \\
\hline $5 d^{5} 6 s^{2}$ & $a{ }^{2} \mathrm{I}$ & $\begin{array}{l}51 / 2 \\
61 / 2\end{array}$ & $\begin{array}{l}23956.00 \\
26348.96\end{array}$ & 2392.96 & $\begin{array}{l}0.995 \\
1.100\end{array}$ & & & $\begin{array}{l}21 / \frac{2}{2} \\
21 / 2\end{array}$ & $\begin{array}{l}37697.66 \\
39670.52\end{array}$ & & $\begin{array}{l}1.219 \\
1.107\end{array}$ \\
\hline $5 d^{5} 6 s^{2}$ & $a^{4} \mathrm{~F}$ & $\begin{array}{l}11 / 2 \\
21 / 2 \\
31 / 2 \\
4^{1 / 2}\end{array}$ & $\begin{array}{l}26131.57 \\
28030.32 \\
28542.13 \\
27514.31\end{array}$ & $\begin{array}{r}1898.75 \\
511.81 \\
-1027.82\end{array}$ & $\begin{array}{l}0.650 \\
1.12 \\
1.13\end{array}$ & $5 d^{5} 6 s\left(a^{5} \mathrm{~S}\right) 6 p$ & $x^{6} \mathrm{P}^{\circ}$ & $\begin{array}{l}31 / 2 \\
21 / 2 \\
11 / 2 \\
11 / 2\end{array}$ & $\begin{array}{l}39844.75 \\
43569.36 \\
43 \% 02.20 \\
40808.85\end{array}$ & $\begin{array}{r}-3724.61 \\
-132.84\end{array}$ & $\begin{array}{l}1.223 \\
1.504 \\
1.962 \\
1.351\end{array}$ \\
\hline $5 d^{6}\left({ }^{3} \mathrm{P}\right) 6 s$ & $b{ }^{4} \mathrm{P}$ & $\begin{array}{l}21 / 2 \\
11 / 2 \\
01 / 2\end{array}$ & $\begin{array}{l}26661.43 \\
30526.60\end{array}$ & -3865.17 & 1. 32 & $5 d^{5} 6 s\left(a^{5} \mathrm{P}\right) 6 p$ & $y{ }^{6} \mathrm{D}^{\circ}$ & $\begin{array}{l}01 / 2 \\
21 / 2 \\
4^{1 / 2} / 2\end{array}$ & $\begin{array}{l}40810.17 \\
40821.83\end{array}$ & & 1. 126 \\
\hline $5 d^{6}\left({ }^{3} \mathrm{H}\right) 6 s^{3}$ & $a{ }^{4} \mathrm{H}$ & $\begin{array}{l}61 / 2 \\
51 / 2 \\
41 / 2 \\
31 / 2\end{array}$ & $\begin{array}{l}27130.14 \\
27243.88 \\
27161.35 \\
28809.87\end{array}$ & $\begin{array}{r}113.74 \\
82.53 \\
1648.52\end{array}$ & $\begin{array}{l}1.208 \\
1.18 \\
0.93\end{array}$ & & & $\begin{array}{l}31 / 2 \\
21 / 2 \\
11 / 2 \\
01 / 2\end{array}$ & $\begin{array}{l}40946.53 \\
41319.02 \\
41557.08\end{array}$ & $\begin{array}{l}-366.49 \\
-244.06\end{array}$ & $\begin{array}{l}1.534 \\
1.278 \\
1.655\end{array}$ \\
\hline $5 d^{6}(5 \mathrm{D}) 6 s$ & $b{ }^{4} \mathrm{D}$ & $\begin{array}{l}31 / 2 \\
21 / 2 \\
11 / 2\end{array}$ & $\begin{array}{l}27141.13 \\
29800.38 \\
27827.65\end{array}$ & $\begin{array}{r}-2659.25 \\
1972.73 \\
442.85\end{array}$ & $\begin{array}{l}1.34 \\
1.17 \\
0.888\end{array}$ & & & $\begin{array}{l}31 / 2 \\
41 / 2 \\
31 / 2\end{array}$ & $\begin{array}{l}41163.91 \\
41453.18 \\
41843.85\end{array}$ & & $\begin{array}{l}1.325 \\
1.372 \\
1.190\end{array}$ \\
\hline $5 d^{5} 6 s\left(a^{7} \mathrm{~S}\right) 6 p$ & $z^{6} \mathrm{P}^{\circ}$ & $\begin{array}{l}01 / 2 \\
31 / 2 \\
2^{1} / 2 \\
11 / 2\end{array}$ & $\begin{array}{l}27384.80 \\
28889.72 \\
28854.18 \\
28961.55\end{array}$ & $\begin{array}{r}35.54 \\
-107.37\end{array}$ & $\begin{array}{r}.072 \\
1.709 \\
1.871 \\
2.333\end{array}$ & $5 d^{5} 6 s\left(a{ }^{5} \mathrm{P}\right) 6 p$ & $z^{4} \mathrm{D}^{\circ}$ & $\begin{array}{l}01 / 2 \\
11 / 2 \\
21 / 2 \\
31 / 2\end{array}$ & $\begin{array}{l}41991.56 \\
42254.19 \\
43044.02 \\
43407.87\end{array}$ & $\begin{array}{l}262.63 \\
789.83 \\
363.85\end{array}$ & $\begin{array}{l}0.61 \\
1.578 \\
1.44 y \\
1.368\end{array}$ \\
\hline $5 d^{5} 6 s^{2}$ & $a^{2} \mathrm{~S}$ & $01 / 2$ & 30131.57 & & & & & $41 / 2$ & 42140.06 & & 1. 249 \\
\hline $5 d^{5} 6 s^{2}$ & $a^{2} \mathrm{H}$ & $\begin{array}{l}51 / 2 \\
41 / 2\end{array}$ & $\begin{array}{l}30559.91 \\
31399.30\end{array}$ & -839.39 & 1.07 & $5 d^{5} 6 s\left(a^{7} \mathrm{~S}\right) 7 s$ & $e^{8 \mathrm{~S}}$ & $\begin{array}{l}31 / 2 \\
11 / 2\end{array}$ & $\begin{array}{l}42598.27 \\
43341.85\end{array}$ & & $\begin{array}{l}1.957 \\
0.975\end{array}$ \\
\hline $5 d^{6}\left({ }^{3} \mathrm{~F}\right) 6 s$ & $b^{4} \mathrm{~F}$ & $\begin{array}{l}11 / 2 \\
21 / 2 \\
31 / 2 \\
41 / 2\end{array}$ & $\begin{array}{l}31460.62 \\
31186.08 \\
30645.33\end{array}$ & -274.54 & 1.17 & & & $\begin{array}{l}11 / 2 \\
41 / 2 \\
31 / 2 \\
21 / 2 \\
51 / 2\end{array}$ & $\begin{array}{l}43409.11 \\
43453.30 \\
43815.01 \\
43949.98 \\
43996.30\end{array}$ & & $\begin{array}{l}1.336 \\
1.348 \\
1.385 \\
1.26\end{array}$ \\
\hline $5 d^{5} 6 s^{2}$ & $b{ }^{2} \mathrm{~F}$ & $\begin{array}{l}31 / 2 \\
2^{1} / 2\end{array}$ & $\begin{array}{l}31982.99 \\
32435.14\end{array}$ & -452.15 & & & & $\begin{array}{l}21 / 2 \\
11 / 2\end{array}$ & $\begin{array}{l}44054.21 \\
44148.45\end{array}$ & & 1. 573 \\
\hline
\end{tabular}


TABLE 4. Electron configurations and spectral terms of Re I-Continued

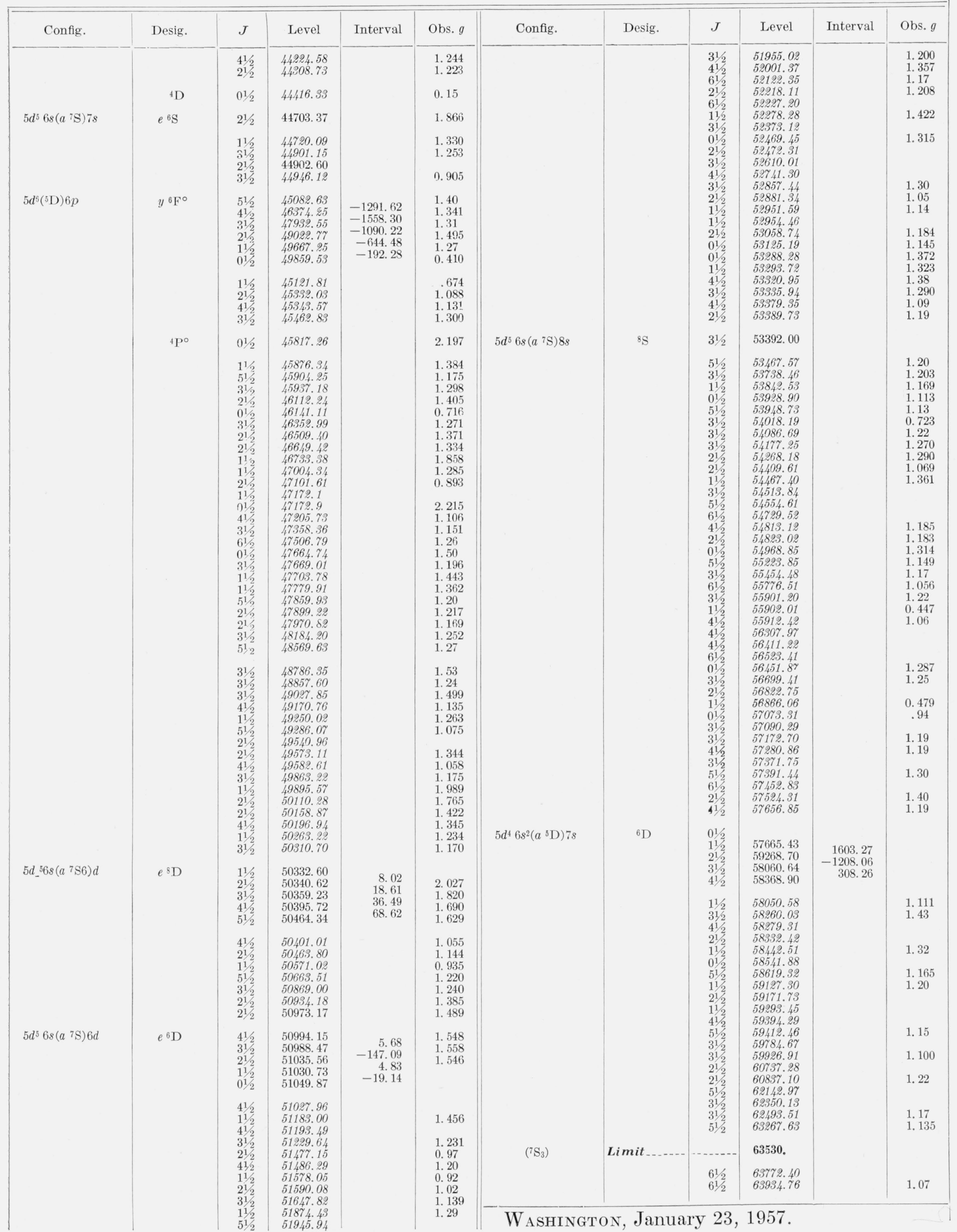

